This is a self-archived version of an original article. This version may differ from the original in pagination and typographic details.

Author(s): Geiss, Stefan; Ylinen, Juha

Title: Decoupling on the Wiener Space, Related Besov Spaces, and Applications to BSDEs

Year: 2021

Version: Accepted version (Final draft)

Copyright: (C) American Mathematical Society, 2019

Rights: $C C B Y-N C-N D 4.0$

Rights url: https://creativecommons.org/licenses/by-nc-nd/4.0/

Please cite the original version:

Geiss, S., \& Ylinen, J. (2021). Decoupling on the Wiener Space, Related Besov Spaces, and Applications to BSDEs. Memoirs of the American Mathematical Society, 272(1335), 1-112. https://doi.org/10.1090/memo/1335 


\title{
Decoupling on the Wiener Space, Related Besov Spaces, and Applications to BSDEs
}

\author{
Stefan Geiss \\ Juha Ylinen
}

\begin{abstract}
Author address:
University of Jyväskylä, Department of Mathematics and Statistics, P.O.Box 35, FI-40014 University of JyVÄSkylä, Finland

E-mail address: stefan.geiss@jyu.fi

University of Jyväskylä, Department of Mathematics and Statistics, P.O.Box 35, FI-40014 University of JyVÄskylä, Finland

E-mail address: juha.m.ylinen@jyu.fi
\end{abstract}


7 Nov 2017 05:35:26 EST

Prob+Stat

Version 2 - Submitted to Memoirs of the AMS 


\section{Contents}

Chapter 1. Introduction 1

1.1. Background 1

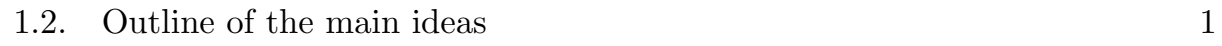

$\begin{array}{lll}1.3 . & \text { Notation } & 10\end{array}$

$\begin{array}{lll}\text { Chapter 2. A General Factorization } & 13\end{array}$

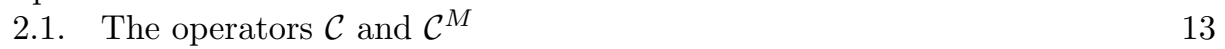

$\begin{array}{lll}2.2 . & \text { The operators } \mathcal{C} \text { and } \mathcal{C}^{M} \text { for stochastic processes } & 17\end{array}$

\begin{tabular}{lll}
\hline Chapter 3. Transference of SDEs & 21
\end{tabular}

3.1. Setting 21

$\begin{array}{lll}3.2 . & \text { Results } & 22\end{array}$

Chapter 4. Anisotropic Besov Spaces on the Wiener Space 25

4.1. Classical Besov spaces on the Wiener space 25

$\begin{array}{lll}4.2 . & \text { Setting } & 28\end{array}$

$\begin{array}{lll}4.3 . & \text { Definition of anisotropic Besov spaces } & 29\end{array}$

$\begin{array}{lll}4.4 . & \text { Connection to real interpolation } & 32\end{array}$

$\begin{array}{llll}4.5 . & \text { The space } \mathbb{B}_{p}^{\Phi_{2}} & 35\end{array}$

4.6. An embedding theorem for functionals of bounded variation 41

4.7. Examples 42

\begin{tabular}{lll}
\hline Chapter 5. Continuous BMO-Martingales & 47
\end{tabular}

5.1. Continuous BMO-martingales and sliceable numbers 47

$\begin{array}{lll}5.2 . & \text { Fefferman's inequality and } \operatorname{BMO}\left(S_{2 \theta}\right) \text { spaces } & 50\end{array}$

\begin{tabular}{ll|l}
5.3. & Reverse Hölder inequalities & 58
\end{tabular}

$\begin{array}{lll}5.4 . & \text { An application to BSDEs } & 60\end{array}$

$\begin{array}{lll}\text { Chapter 6. Applications to BSDEs } & 65\end{array}$

6.1. The setting 65

6.2. Stability of BSDEs with respect to perturbations of the Gaussian

\begin{tabular}{lll}
\hline 6.3. & On classes of quadratic and sub-quadratic BSDEs & 72
\end{tabular}

\begin{tabular}{lll}
\hline 6.4. & Settings for the stability theorem & 80
\end{tabular}

6.5. On the $L_{p}$-variation of BSDEs 86

\begin{tabular}{lll}
\hline 6.6. & Applications to other types of BSDEs & 92
\end{tabular}

\begin{tabular}{lll}
\hline Appendix A. Technical Facts & 95
\end{tabular}

\begin{tabular}{lll}
\hline Appendix. Bibliography & 105
\end{tabular} 
\begin{tabular}{lll}
\hline Appendix. Index & 109
\end{tabular}

7 Nov 2017 05:35:26 EST

Prob+Stat

Version 2 - Submitted to Memoirs of the AMS 


\begin{abstract}
We introduce a decoupling method on the Wiener space to define a wide class of anisotropic Besov spaces. The decoupling method is based on a general distributional approach and not restricted to the Wiener space.

The class of Besov spaces we introduce contains the traditional isotropic Besov spaces obtained by the real interpolation method, but also new spaces that are designed to investigate backwards stochastic differential equations (BSDEs). As examples we discuss the Besov regularity (in the sense of our spaces) of forward diffusions and local times. It is shown that among our newly introduced Besov spaces there are spaces that characterize quantitative properties of directional derivatives in the Malliavin sense without computing or accessing these Malliavin derivatives explicitly.

Regarding BSDEs, we deduce regularity properties of the solution processes from the Besov regularity of the initial data, in particular upper bounds for their $L_{p^{-}}$ variation, where the generator might be of quadratic type and where no structural assumptions, for example in terms of a forward diffusion, are assumed. As an example we treat sub-quadratic BSDEs with unbounded terminal conditions.

Among other tools, we use methods from harmonic analysis. As a by-product, we improve the asymptotic behaviour of the multiplicative constant in a generalized Fefferman inequality and verify the optimality of the bound we established.
\end{abstract}

2010 Mathematics Subject Classification. Primary 60H07, 60H10, $46 \mathrm{E} 35$.

Key words and phrases. Anisotropic Besov spaces, Decoupling on the Wiener Space, Backward Stochastic Differential Equations, Interpolation.

The authors were supported by the project "Stochastic and Harmonic Analysis, interactions, and applications", No. 133914, of the Academy of Finland. The second author was partly supported by the Vilho, Yrjö and Kalle Väisälä foundation of the Finnish Academy of Science and Letters. 
7 Nov 2017 05:35:26 EST

Prob+Stat

Version 2 - Submitted to Memoirs of the AMS 


\section{CHAPTER 1}

\section{Introduction}

\subsection{Background}

A backward stochastic differential equation (BSDE) is an equation of type

$$
Y_{t}=\xi+\int_{t}^{T} f\left(s, Y_{s}, Z_{s}\right) d s-\int_{t}^{T} Z_{s} d W_{s},
$$

where $T>0$ is a fixed finite time horizon, $W=\left(W_{t}\right)_{t \in[0, T]}$ is a $d$-dimensional Brownian motion, $\xi: \Omega \rightarrow \mathbb{R}$ is a given $\mathcal{F}_{T}$-measurable terminal condition, and

$$
f:[0, T] \times \Omega \times \mathbb{R} \times \mathbb{R}^{d} \rightarrow \mathbb{R}
$$

is a given predictable random generator which might be non-Markovian. Given the data $(\xi, f)$, one looks for adapted solution processes $(Y, Z)$. Backward stochastic differential equations have a wide range of applications, for example in stochastic control and, more generally, in stochastic modeling. In the case of a Markovian generator, where the randomness comes from a forward diffusion, there is an important and extremely useful connection to non-linear partial differential equations of parabolic type, the so-called (non-linear) Feynman-Kac theory. Two seminal papers in this theory were the work of Bismut [13, and Pardoux and Peng [73.

The simulation of BSDEs is an important topic and subject to active research. To setup simulation schemes one needs an approximation theory for BSDEs, for example to find optimal time-grids or to obtain upper and lower rates for the speed of convergence of these schemes measured in an appropriate way. To investigate these approximation properties it is more or less mandatory to understand the variational properties of the solution $(Y, Z)$, i.e. the behavior of

$$
\left\|Y_{t}-Y_{s}\right\|_{p} \quad \text { and (say) } \quad\left\|\left(\int_{s}^{t}\left|Z_{r}\right|^{2} d r\right)^{\frac{1}{2}}\right\|_{p}
$$

for all $0 \leq s<t \leq T$ and an appropriate range of $p \in(0, \infty)$, where $\|\xi\|_{p}:=$ $\|\xi\|_{L_{p}(\Omega)}=\left(\mathbb{E}|\xi|^{p}\right)^{\frac{1}{p}}$ for a random variable $\xi: \Omega \rightarrow \mathbb{R}$.

\subsection{Outline of the main ideas}

In these notes we develop an approach to estimate the variations from (2) in terms of the regularity of the data $(\xi, f)$, where the regularity is a fractional smoothness expressed in terms of Besov spaces. Our approach is based on an anisotropic decoupling of the Wiener space. Recently this decoupling was already successfully used in [40, 41] and constitutes one of the few approaches to estimate variational properties of non-Markovian backwards equations using only knowledge of the initial data. Let us explain the basic line of ideas to motivate the structure of these notes. 
If the generator in our BSDE vanishes, i.e. $f \equiv 0$, then one has that

$$
Y_{t}=\mathbb{E}\left(\xi \mid \mathcal{F}_{t}\right) \text {. }
$$

Therefore, in the case $f \not \equiv 0$ the map

$$
G_{t}^{f}: \xi \rightarrow Y_{t}
$$

can be interpreted as some kind of generalized non-linear conditional expectation along the generator $f$ (see $[\mathbf{7 4}, \mathbf{2 9}, \mathbf{7 5}$ for the notion of $g$-expectation and nonlinear expectations). It turns out that our notion of regularity is stable with respect to this non-linear map $G_{t}^{f}$. Moreover, since

$$
\left\|Y_{t}-Y_{s}\right\|_{p} \leq\left\|Y_{t}-\mathbb{E}\left(Y_{t} \mid \mathcal{F}_{s}\right)\right\|_{p}+\left\|\mathbb{E}\left(Y_{t} \mid \mathcal{F}_{s}\right)-Y_{s}\right\|_{p}
$$

and since $\left\|\mathbb{E}\left(Y_{t} \mid \mathcal{F}_{s}\right)-Y_{s}\right\|_{p}$ can be handled by 'standard' methods, the main question consists in investigating the behavior of $\left\|Y_{t}-\mathbb{E}\left(Y_{t} \mid \mathcal{F}_{s}\right)\right\|_{p}$ for $s \uparrow t$. It turns out that this behaviour corresponds to a notion of fractional smoothness in $L_{p}$ of the random variable $Y_{t}$. The crucial point here is that

$$
\left\|Y_{t}-\mathbb{E}\left(Y_{t} \mid \mathcal{F}_{s}\right)\right\|_{p} \sim\left\|Y_{t}-Y_{t}^{(s, t]}\right\|_{p}
$$

for $p \in[1, \infty)$, where $Y_{t}^{(s, t]}$ is a decoupled version of $Y_{t}$ in the sense explained below. Therefore we proceed as follows:

(a) In Chapter 2 we introduce a factorization and a method to transfer stochastic processes from one stochastic basis to another one while keeping distributional and measurability properties.

(b) In Chapter 3 we apply the methods from Chapter 2 to the Wiener space, in particular to stochastic differential equations driven by the Brownian motion.

(c) In Chapter 4 the decoupling and the corresponding Besov spaces on the Wiener space are introduced and investigated.

(d) In Chapter 5 we provide some tools about BMO spaces and reverse Hölder inequalities and apply them to non-Lipschitz BSDEs.

(e) In Chapter 6 we apply further the results of Chapters 2, 3, 4, and 5 to BSDEs.

We proceed with some exemplary ideas and results obtained in this article:

Chapters 2 - 4: The decoupling to obtain $F^{(a, b]}$ from a random variable $F: \Omega \rightarrow$ $\mathbb{R}$ on the Wiener space is done as follows: We start with a Wiener space built on a $d$-dimensional Brownian motion $W=\left(W_{t}\right)_{t \in[0, T]}$. Then we take a copy of this Wiener space, denote the corresponding Brownian motion by $W^{\prime}=\left(W_{t}^{\prime}\right)_{t \in[0, T]}$, and form the canonical product space carrying the $2 d$-dimensional Brownian motion $\left(\left(W_{t}, W_{t}^{\prime}\right)\right)_{t \in[0, T]}$. But the pair $\left(W, W^{\prime}\right)$ of Brownian motions is not the one we are interested in in the sequel. Instead, we take (for example) an interval $(a, b] \subset$ $(0, T]$ and consider the mixed Brownian motion $W^{(a, b]}=\left(W_{t}^{(a, b]}\right)_{t \in[0, T]}$ where the increments on the interval $(a, b]$ from $W$ are replaced by the increments of the independent copy $W^{\prime}=\left(W_{t}^{\prime}\right)_{t \in[0, T]}$, i.e. we define

$$
W_{t}^{(a, b]}=\left\{\begin{array}{rl}
W_{t} & : \quad 0 \leq t \leq a \\
W_{a}+W_{t}^{\prime}-W_{a}^{\prime} & : \quad a \leq t \leq b \\
W_{a}+\left(W_{b}^{\prime}-W_{a}^{\prime}\right)+\left(W_{t}-W_{b}\right) & : \quad b \leq t \leq T
\end{array} .\right.
$$

In other words, the Gaussian structure on $(a, b]$ is replaced by an independent copy: 


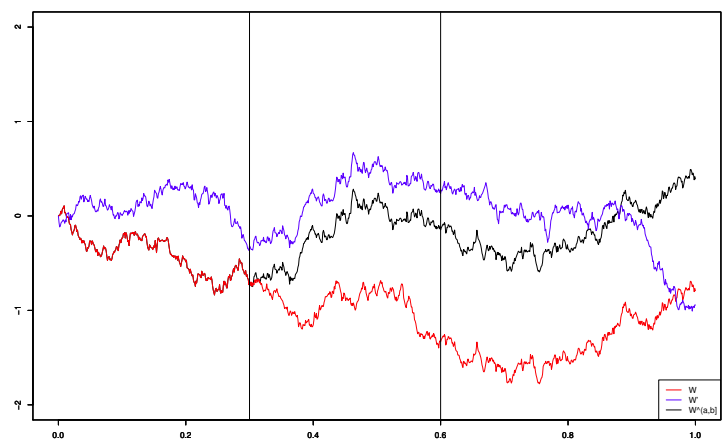

Now the random variables $F$ from the original Wiener space built on $W$ are extended to the product space carrying $\left(W, W^{\prime}\right)$ and are transformed by a functional mapping $F \rightarrow F^{(a, b]}$ along the same map as $W \rightarrow W^{(a, b]}$ is transformed.

After we have introduced the decoupling method, our next step consists in observing that one can define anisotropic Besov spaces by imposing Hölder type conditions on a random variable $\xi \in L_{p}$ like

$$
\left\|\xi-\xi^{(a, b]}\right\|_{p} \leq c \alpha(a, b)
$$

for all $0 \leq a<b \leq T$ and an appropriate weight function $\alpha(\cdot, \cdot)$. These anisotropic Besov spaces are part of a wider class of spaces containing the traditional Besov spaces obtained by the real interpolation method. To explain the diction anisotropic, let us assume $d=1$ and let us formally write $\xi=f(W)$ for an appropriate functional $f: C[0, T] \rightarrow \mathbb{R}$. If $\emptyset \neq(a, b] \neq(0, T]$, then in 4 we compare $f(W)$ with $f\left(W^{(a, b]}\right)$ and note that there is no constant $c \in[0,1]$ such that

$$
\mathbb{E} W_{s} W_{t}^{(a, b]}=c \mathbb{E} W_{s} W_{t} \quad \text { for all } \quad s, t \in[0, T] .
$$

Now let $\theta \in(0,1)$ and define the Brownian motion $W^{\theta}$ by

$$
W_{t}^{\theta}:=\sqrt{1-\theta^{2}} W_{t}+\theta W_{t}^{\prime} \quad \text { for } \quad t \in[0, T] .
$$

Here the Brownian motion $W^{\theta}$ (partially) decouples $W$ uniformly in time, not only on $(a, b]$. This means, that we have an isotropic decoupling. In contrast to (4), the expression $\left\|f(W)-f\left(W^{\theta}\right)\right\|_{p}$ compares $f(W)$ and $f\left(W^{\theta}\right)$, where

$$
\mathbb{E} W_{s} W_{t}^{\theta}=\sqrt{1-\theta^{2}} \mathbb{E} W_{s} W_{t} \text { for all } s, t \in[0, T] .
$$

The reader is also referred to Remark 4.21 below for a more detailed example of being anisotropic.

To explain a prototype of our Besov spaces, let us assume $p, r \in[2, \infty)$ and $\xi \in L_{p}$. In Chapter 4 we use inequality 4 with

$$
\alpha_{r}(a, b):=\sqrt[r]{b-a}
$$

to define $\xi \in \mathbb{B}_{p}^{\Phi_{r}}$ provided that

$$
\|\xi\|_{\mathbb{B}_{p}^{\Phi_{r}}}^{p}:=\mathbb{E}|\xi|^{p}+\|\xi\|_{\Phi_{r}, p}^{p}<\infty \quad \text { with } \quad\|\xi\|_{\Phi_{r}, p}^{p}:=\sup _{0 \leq a<b \leq T}\left|\frac{\left\|\xi-\xi^{(a, b]}\right\|_{p}}{\sqrt[r]{b-a}}\right|^{p} .
$$


The case $r=2$ is treated by Theorem 4.22 and includes the following situation, where $\mathbb{D}_{1,2}$ stands for the Malliavin Sobolev space and $D \xi$ for the Malliavin derivative:

Theorem 1.1. One has $\mathbb{B}_{2}^{\Phi_{2}} \subseteq \mathbb{D}_{1,2}$. Moreover, for $p \in[2, \infty)$ and $\xi \in \mathbb{D}_{1,2} \cap L_{p}$ it holds

$$
\|\xi\|_{\Phi_{2}, p} \sim_{c} \sup _{0 \leq a<b \leq T}\left\|\left(\frac{1}{b-a} \int_{a}^{b}\left|D_{s} \xi\right|^{2} d s\right)^{\frac{1}{2}}\right\|_{p},
$$

where $c>0$ depends on $p$ only. In particular, for $p=2$ we have that

$$
\|\xi\|_{\Phi_{2}, 2} \sim_{c} \operatorname{esssup}_{s \in[0, T]}\left\|D_{s} \xi\right\|_{2} .
$$

The impact of Theorem 1.1 (Theorem 4.22) is at least twofold: Firstly, we can access the Malliavin derivative by the spaces $\mathbb{B}_{p}^{\Phi_{2}}$ without using the derivative explicitly. Secondly, the above theorem can be localized by replacing $\|\xi\|_{\mathbb{B}_{p}^{\Phi_{2}}}^{p}$ with

$$
\mathbb{E}|\xi|^{p}+\sup _{A \leq a<b \leq B}\left|\frac{\left\|\xi-\xi^{(a, b]}\right\|_{p}}{\sqrt{b-a}}\right|^{p}
$$

for some $0 \leq A<B \leq T$. Here $\xi$ does not need to belong to $\mathbb{D}_{1,2}$ anymore.

The case $r=4$ turns out to be relevant for the local time of a Brownian motion, for example represented by

$$
L_{t}^{\alpha}=\lim _{\varepsilon \downarrow 0} \frac{1}{2 \varepsilon} \int_{0}^{t} \chi_{(\alpha-\varepsilon, \alpha+\varepsilon)}\left(W_{s}\right) d s \text { a.s. }
$$

We prove in Corollary 4.30 that for all $\alpha \in \mathbb{R}$ and $p \in(1, \infty)$ one has that

$$
L_{T}^{\alpha} \in \mathbb{B}_{p}^{\Phi_{4}} \backslash\left[\bigcup_{r \in[2,4)} \mathbb{B}_{p}^{\Phi_{r}}\right] .
$$

BACKGRound AND RELATED RESUlts: Our method includes with Theorem 4.16 a characterization by decoupling of the real interpolation spaces $\left(L_{p}, \mathbb{D}_{1, p}\right)_{\theta, q}$ for the full range of interpolation parameters $(\theta, q) \in(0,1) \times[1, \infty]$, where $p \in[2, \infty)$. This directly extends [46. Theorem 3.1] to the case that the supporting Hilbert space of the Gaussian structure of the abstract Wiener space is infinite dimensional. In [50, Remark on p. 428] a different characterization by decoupling was given in the case $p=q$, i.e. for $\left(L_{p}, \mathbb{D}_{1, p}\right)_{\theta, p}$. The case $q \neq p$ is of natural interest on its own, but the full range of parameters $(\theta, q) \in(0,1) \times[1, \infty]$ is also crucial for the understanding of certain phenomena in applications.

The idea to use decoupling to understand better Malliavin Sobolev spaces was used before: The natural question, whether Malliavin Sobolev spaces are stable under Lipschitz mapping has been raised by Watanabe in $\mathbf{8 8}$ and answered by Hirsch $\mathbf{5 0}$ by describing $\left(L_{p}, \mathbb{D}_{1, p}\right)_{\theta, p}$ by decoupling. Roughly speaking, any representation by decoupling is stable under Lipschitz mappings, so our Besov spaces $\mathbb{B}_{p}^{\Phi}$ are stable. Therefore Theorem 4.16 below verifies as a by-product that the spaces $\left(L_{p}, \mathbb{D}_{1, p}\right)_{\theta, q}$ are stable under Lipschitz mappings for all $(\theta, q) \in(0,1) \times[1, \infty]$ and $p \in[2, \infty)$. 
Chapter 5: Given a continuous BMO-martingale $M$ and its Doléan-Dade exponential $\mathcal{E}(M)$, we introduce the sliceable numbers $\operatorname{sl}_{N}(M)$, that measure the distance of $M$ to $\mathbb{H}_{\infty}$, in Definition 5.2. Here $\mathbb{H}_{\infty}$ stands for the space of all continuous mean zero martingales $N$ with $\|N\|_{\mathbb{H}_{\infty}}:=\|\langle N\rangle\|_{\infty}<\infty$ (see Definition 5.4. Denoting by $\mathcal{R} \mathcal{H}_{\beta}(\mathcal{E}(M))$ the constant in the reverse Hölder inequality for $\mathcal{E}(M)$ with the exponent $\beta$, we prove in Theorem 5.25 .

TheOREM 1.2. Let $\Phi:(1, \infty) \rightarrow(0, \infty)$ be a non-increasing function and let

$$
\Psi:\{(\gamma, \beta) \in[0, \infty) \times(1, \infty): 0 \leq \gamma<\Phi(\beta)<\infty\} \rightarrow[0, \infty)
$$

be right-continuous in its first argument and such that

$$
\Psi\left(\gamma_{1}, \beta\right) \leq \Psi\left(\gamma_{2}, \beta\right) \quad \text { for } \quad 0 \leq \gamma_{1} \leq \gamma_{2}<\Phi(\beta),
$$

with the property that $\|M\|_{\mathrm{BMO}}<\Phi(\beta)$ implies $\mathcal{R H}_{\beta}(\mathcal{E}(M)) \leq \Psi\left(\|M\|_{\mathrm{BMO}}, \beta\right)$. Then, for $\operatorname{sl}_{N}(M)<\Phi(\beta)$ we have that

$$
\mathcal{R H}_{\beta}(\mathcal{E}(M)) \leq\left[\Psi\left(\operatorname{sl}_{N}(M), \beta\right)\right]^{N} .
$$

The point of this observation is that we get explicit exponents $\beta$ and explicit bounds for $\mathcal{R H}_{\beta}(\mathcal{E}(M))$ in terms of the sliceable numbers $\left(\operatorname{sl}_{N}(M)\right)_{N \geq 1}$. This is applied to BMO-martingales obtained by the fractional gradient $|Z|^{\theta}$ of our BSDE where $\theta \in[0,1]$ is the parameter from (5) below that describes the degree of the BSDEs of not being Lipschitz in the $Z$-component $(\theta=0$ corresponds to the Lipschitz case, $\theta=1$ to the quadratic case).

Another contribution concerns the generalized Fefferman inequality [32, Lemma 1.6] (see also [5, Theorem 1.1]). We prove with Theorem 5.18 a more abstract version using adapted random measures that yields in Corollary 5.19 to

$$
\left\|\int_{0}^{T}\left|A_{t} B_{t}\right| d t\right\|_{p} \leq \sqrt{2 p}\|A\|_{\mathbb{H}_{p}\left(S_{2}\right)}\|B\|_{\mathrm{BMO}\left(S_{2}\right)}
$$

which improves the asymptotic behavior of the constant from $p$ in 32 to $\sqrt{p}$. We also verify that the asymptotic order $\sqrt{p}$ as $p \rightarrow \infty$ is optimal.

Chapter 6 The decoupling method for BSDEs originates from [40, where the terminal condition did depend on finitely many increments of a forward diffusion and the generator was Markovian and Lipschitz. The aim of this part of the notes is the further development of this method. Motivated by the equivalence (3) we first decouple the BSDE (1) in order to get a new BSDE

$$
Y_{t}^{(a, b]}=\xi^{(a, b]}+\int_{t}^{T} f^{(a, b]}\left(s, Y_{s}^{(a, b]}, Z_{s}^{(a, b]}\right) d s-\int_{t}^{T} Z_{s}^{(a, b]} d W_{s}^{(a, b]}
$$

and aim to use a priori estimates for BSDEs to estimate $\left\|\sup _{s \in[t, T]}\left|Y_{s}^{(a, b]}-Y_{s}\right|\right\|_{p}$ and $\left\|\left(\int_{t}^{T}\left|Z_{s}^{(a, b]}-Z_{s}\right|^{2} d s\right)^{\frac{1}{2}}\right\|_{p}$ from above by moments of

$$
\xi-\xi^{(a, b]} \text { and } f-f^{(a, b]} .
$$


Here we consider generators $f:[0, T] \times \Omega \times \mathbb{R} \times \mathbb{R}^{d} \rightarrow \mathbb{R}$ such that $(t, \omega) \mapsto$ $f(t, \omega, y, z)$ is predictable for all $(y, z)$ and there are $L_{Y}, L_{Z} \geq 0$ and $\theta \in[0,1]$ such that

$$
\left|f\left(t, \omega, y_{0}, z_{0}\right)-f\left(t, \omega, y_{1}, z_{1}\right)\right| \leq L_{Y}\left|y_{0}-y_{1}\right|+L_{Z}\left[1+\left|z_{0}\right|+\left|z_{1}\right|\right]^{\theta}\left|z_{0}-z_{1}\right|
$$

for all $\left(t, \omega, y_{0}, y_{1}, z_{0}, z_{1}\right)$. Here $\theta=0$ represents the Lipschitz case, $\theta=1$ the quadratic case, and $\theta \in(0,1)$ the sub-quadratic case. The basic stability result is Theorem 6.3 , a special case is:

TheOrem 1.3. Assume for the BSDE

$$
Y_{t}=\xi+\int_{t}^{T} f\left(s, Y_{s}, Z_{s}\right) d s-\int_{t}^{T} Z_{s} d W_{s}, \quad t \in[0, T],
$$

conditions (B1)-(B4) of Chapter 6 for $\theta \in[0,1]$ and that there is a non-increasing sequence $\left(s_{N}\right)_{N>1} \subseteq[0, \infty)$ which dominates the sliceable numbers of the fractional gradient, i.e. $\mathrm{sl}_{N}^{\bar{S}_{2}}\left(|Z|^{\theta}\right) \leq s_{N}$ for all $N \geq 1$. Suppose that conditions (B5)-(B6) of Chapter 6 are satisfied for $p \in[2, \infty)$ where in the case $\lim _{N} s_{N}>0$ we additionally assume that $p>p_{0}\left(L_{Z}, \lim _{N} s_{N}\right)$. Then, one has for all $t \in[0, T]$ and $0 \leq a<b \leq$ T that

$$
\begin{aligned}
\left\|\sup _{s \in[t, T]}\left|Y_{s}^{(a, b]}-Y_{s}\right|\right\|_{p}+\left\|\left(\int_{t}^{T}\left|Z_{s}^{(a, b]}-Z_{s}\right|^{2} d s\right)^{\frac{1}{2}}\right\|_{p} \\
\leq c\left[\left\|\xi^{(a, b]}-\xi\right\|_{p}+\left\|\int_{t}^{T}\left|f^{(a, b]}\left(s, Y_{s}, Z_{s}\right)-f\left(s, Y_{s}, Z_{s}\right)\right| d s\right\|_{p}\right] .
\end{aligned}
$$

In order to apply Theorem 1.3 (Theorem 6.3), and because of general interest, we discuss classes of quadratic and sub-quadratic BSDEs such that the assumptions of Theorems 1.3 and 6.3 are satisfied in Section 6.3. In case of sub-quadratic BSDEs we use the following definition:

\section{DEFINITION 1.4.}

(1) We say that a random variable $\xi$ belongs to cExp provided that there are $(\eta, \mu) \in(0,1) \times(0, \infty)$ such that

$$
|\xi|_{\operatorname{cExp}(\eta, \mu)}:=\sup _{t \in[0, T)}(T-t)^{\frac{1}{\eta}-1}\left\|\mathbb{E}\left(e^{\mu|\xi|} \mid \mathcal{F}_{t}\right)\right\|_{\infty}<\infty .
$$

(2) For a càdlàg process $Y=\left(Y_{t}\right)_{t \in[0, T]}$ we say that $Y \in \mathrm{cExp}$ provided that there are $(\eta, \mu) \in(0,1) \times(0, \infty)$ such that

$$
|Y|_{\operatorname{cExp}(\eta, \mu)}:=\sup _{t \in[0, T)}(T-t)^{\frac{1}{\eta}-1}\left\|\mathbb{E}\left(e^{\mu \sup _{s \in[t, T]}\left|Y_{s}\right|} \mid \mathcal{F}_{t}\right)\right\|_{\infty}<\infty .
$$

In Theorem 6.13 we prove the following statement: 
Theorem 1.5. Assume (5) for some $\theta \in(0,1), \sup _{(t, \omega) \in[0, T] \times \Omega}|f(t, \omega, 0,0)|<\infty$, and that $\xi \in \mathrm{cExp}$. Then there is a unique solution $(Y, Z)$ to the $\mathrm{BSDE}(1)$ in the class where $(Y,|Z|) \in \mathrm{cExp} \times \mathbb{H}_{2}\left(\mathrm{~S}_{2}\right)$ 1. Moreover, for this solution we have that

$$
|Z|^{\eta} \in \operatorname{BMO}\left(S_{2}\right) \quad \text { for all } \eta \in(0,1) \text {. }
$$

Theorem 1.5 enables us to apply Theorem 1.3 , so that a combination with Theorem 1.1 gives in Corollary 6.23 .

Corollary 1.6. Assume (5) for some $\theta \in(0,1), \sup _{(s, \omega) \in[0, T] \times \Omega}|f(s, \omega, 0,0)|<$ $\infty, \xi \in \mathrm{cExp}$, and that $(Y, Z)$ is the unique solution to the BSDE (1) in the sense of Theorem 1.5. Fix $t \in[0, T]$. Then we have

$$
\begin{aligned}
& \operatorname{esssup}_{s \in[0, t]}\left\|D_{s} Y_{t}\right\|_{2} \\
& \leq c \sup _{(a, b] \subseteq(0, t]} \frac{1}{\sqrt{b-a}}\left[\left\|\xi-\xi^{(a, b]}\right\|_{2}+\left\|\int_{t}^{T} \sup _{y, z}\left|f(s, y, z)-f^{(a, b]}(s, y, z)\right| d s\right\|_{2}\right]
\end{aligned}
$$

with the convention that the finiteness of the right-hand side first implies $Y_{t} \in \mathbb{D}_{1,2}$ and then inequality (6).

The assertion of Corollary 1.6 says that we only need to control directional derivatives of the initial data $(\xi, f)$ on the interval $(0, t]$ (because the perturbations of the original Brownian motion $W$ are only performed on $(a, b] \subseteq(0, t])$ to obtain smoothness of $Y_{t}$ and that the behaviour of $(\xi, f)$ regarding perturbations on $(t, T]$ does not have any impact - in a sense, we have a smoothing effect.

Finally, let us turn to the $L_{p}$-variation of a solution $(Y, Z)$ to our BSDE. Our idea is to use adapted time-nets obtained by a quantile method. This idea is made precise by the following two definitions:

Definition 1.7. Let $p \in[1, \infty), A=\left(A_{t}\right)_{t \in[0, T]}$ be a measurable càdlàg process $A:[0, T] \times \Omega \rightarrow \mathbb{R}$, and $C=\left(C_{t}\right)_{t \in[0, T]}$ be a measurable process $C:[0, T] \times \Omega \rightarrow$ $\mathbb{R}^{d}$, where $\mathbb{R}^{d}$ is equipped with the Euclidean norm. For a deterministic time-net $\tau=\left(t_{i}\right)_{i=0}^{n}$ with $0=t_{0} \leq t_{1} \leq \cdots \leq t_{n}=T$ we let

$$
\operatorname{var}_{p}([A, C] \mid \tau):=\sup _{i=1, \ldots, n}\left\|\sup _{t_{i-1} \leq s \leq t \leq t_{i}}\left|A_{t}-A_{s}\right|\right\|_{p}+\sup _{i=1, \ldots, n}\left\|\left(\int_{t_{i-1}}^{t_{i}}\left|C_{r}\right|^{2} d r\right)^{\frac{1}{2}}\right\|_{p} .
$$

Definition 1.8. Letting $\Lambda:[0, T] \rightarrow(0, \infty)$ be integrable and $n \geq 1$, the time-net $\tau_{n}^{\Lambda}$ consists of $0=t_{0}<\cdots<t_{n}=T$ such that, for all $i=1, \ldots, n$,

$$
\int_{t_{i-1}}^{t_{i}} \Lambda(r) d r=\frac{1}{n} \int_{0}^{T} \Lambda(r) d r
$$

Now we obtain as part of Corollary 6.32 the following result:

\footnotetext{
${ }^{1}$ The spaces are given in Definitions 6.11 and 5.11 below.
} 
Theorem 1.9. Assume (5) for some $\theta \in(0,1), \sup _{(t, \omega) \in[0, T] \times \Omega}|f(t, \omega, 0,0)|<\infty$, $\gamma \in[2, \infty), \xi \in \mathrm{cExp}$, and that

$$
\left\|\xi-\xi^{(a, b]}\right\|_{2}+\left\|\int_{a}^{T} \sup _{(y, z) \in \mathbb{R}^{d+1}}\left|f(r, y, z)-f^{(a, b]}(r, y, z)\right| d r\right\|_{2} \leq\left(\int_{a}^{b} \Gamma(r) d r\right)^{\frac{1}{\gamma}}
$$

for some integrable Borel function $\Gamma:[0, T] \rightarrow[0, \infty)$. Define the weight function

$$
\Lambda(r):=1+\|f(r, 0,0)\|_{2}+\Gamma(r) .
$$

Then one has that

$$
\sup _{n \geq 1} \sqrt[\gamma]{n} \operatorname{var}_{2}\left([Y, Z] \mid \tau_{n}^{\Lambda}\right)<\infty
$$

where the solution is taken from Theorem 1.5 .

Theorem 1.9 allows us to control the $L_{2}$-variation of non-Markovian BSDEs by adapted time-nets where only the information of the initial data $(\xi, f)$ is used.

BACKGROUnd AND RELATED RESUlts: Because of applications in stochastic modeling and due to the connections to non-linear PDEs, the simulation of BSDEs is of particular importance and subject to active research (see for example [92, 17, 47, 16, 52, 63. in the Lipschitz case, [55, 81, 26] in the quadratic case, and 33 for an overview about various numerical methods related to BSDEs). To setup simulation schemes, one typically considers a time discretization. First, one fixes a deterministic time-grid $\tau=\left(t_{i}\right)_{i=0}^{n}$, where $0=t_{0}<t_{1}<\cdots<t_{n}=T$, and a simulation scheme based on this grid is considered. For the $Y$-process this means that one finds random variables $\left(Y_{t_{i}}^{\tau}\right)_{i=0}^{n}$ that are sampled and provide an approximation of the random variables $\left(Y_{t_{i}}\right)_{i=0}^{n}$. To study how accurate this approximation is, one option is to consider the $L_{p^{-}}$simulation error

$$
\operatorname{err}_{p}(\tau):=\sup _{0 \leq i \leq n}\left\|Y_{t_{i}}-Y_{t_{i}}^{\tau}\right\|_{p}
$$

for certain $p \in[2, \infty)$. For any feasible simulation scheme, the simulation error should go to zero as the mesh-size of the grid goes to zero. Preferably there is even a rate of convergence, which could mean that there exists a $c_{p}>0$, independent of the particular grid $\tau$, such that

$$
\operatorname{err}_{p}(\tau) \leq c_{p}\left(\max _{i=1, \ldots, n}\left|t_{i}-t_{i-1}\right|\right)^{\frac{1}{2}}
$$

To obtain the estimate (7), it turns out to be more or less mandatory to have a path regularity of the exact solution itself. The preferred estimate would be to have some $d_{p}>0$ such that

$$
\left\|Y_{t}-Y_{s}\right\|_{p} \leq d_{p}(t-s)^{\frac{1}{2}}
$$

for any $0 \leq s<t \leq T$, or a variant of this inequality. It is known that upper bounds for the variation $\left\|Y_{t}-Y_{s}\right\|_{p}$ also relate to differential properties of the initial data and how these properties transfer to the solution processes. Let us review parts of the corresponding literature: 
(1) Initial data in $\mathbb{D}_{1,2}$ or of Lipschitz type

Regularity and differential properties: Regarding Lipschitz BSDEs (the generator is Lipschitz in $z$ ) we refer for differential properties of $Y$ and the representation of $Z$ by the Malliavin derivative of $Y$ to $[\mathbf{3 4}, \mathbf{5 2}, \mathbf{6 3}, 42$ and the references therein. The notion of an $L_{\infty}$-Lipschitz functional of a forward diffusion has been used in 92 and 9 . For quadratic BSDEs (the generator only satisfies certain local Lipschitz conditions in $z$ ) general regularity results are given in 2. These general results were applied to Markovian decoupled FBSDEs (in particular, the randomness of the data $(\xi, f)$ of the BSDE is induced by a forward process $\left.\left(X_{t}\right)_{t \in[0, T]}\right)$ in 2 as well. In [27 the existence and uniqueness of solutions to quadratic BSDEs is studied when the terminal condition $\xi$ has a uniformly bounded Malliavin derivative, i.e. $|D . \xi(\cdot)| \leq c$ a.e. which relates to our spaces $\mathbb{B}_{p}^{\Phi_{2}}$ by Theorem 4.22 The existence of solutions to some multidimensional quadratic BSDEs, examining as a special case subquadratic BSDEs, is considered in $\mathbf{2 8}$ under the assumption that the terminal condition is bounded. Continuing with decoupled Markovian FBSDEs under certain Lipschitz assumptions on the terminal condition, variational estimates for $Z$ can be found in $\mathbf{8 1}$ and uniqueness and existence results under conditions on the forward diffusion and the final time horizon $T$ are obtained in 82 .

Variational properties of $Y$ : Typically estimates of type $\left\|Y_{t}-Y_{s}\right\|_{p} \leq$ $c_{p} \sqrt{t-s}$ (and related estimates for the $Z$-process) are obtained for decoupled Markovian FBSDE. So, with terminal values of type $\xi=g\left(X_{T}\right)$ this kind of results can be found in [17. Lemma 3.2], [54, Theorem 5.5] ([54, Lemma 5.1] gives an estimate for $\left.\left\|D_{v} Y_{t}-D_{u} Y_{t}\right\|_{p}\right)$, [55. Theorem 4.4], and [26. Proposition 3.1]. The setting is more general in [92, Lemma 2.3], as there the terminal condition is a path-dependent functional of a forward diffusion. A fully random setting is used in [52, Corollary 2.7].

(2) Markovian decoupled FBSDEs with fractional singularities of different types at the finite time horizon $T$ : To handle approximation problems for stochastic integrals with a singularity at time of maturity, special non-equidistant time-nets have been used in 43 and 39 . In the context of BSDEs this idea and these time-nets have been exploited in 48 and 87 .

(3) Irregular path-dependent terminal conditions: Terminal conditions that depend on finitely many time instances of a forward diffusion and have there local fractional singularities have been considered in [40, the results extend those from 48 .

Now, let us indicate our contribution related to BSDEs:

(1) We improve the comparison theorem [2, Theorem 5.1] in Lemma 5.26 below where we use a generalization of Fefferman's inequality (see Remark 5.27).

(2) Our decoupling method can be directly applied to the above mentioned $L_{\infty^{-}}$ Lipschitz functionals of forward diffusions, as used in 92 and 9]: Assuming such a functional $g(X)$, that depends on finitely many instances of a forward diffusion $X=\left(X_{t}\right)_{t \in[0, T]}$, we directly get the estimate

$$
\left|g(X)-g\left(X^{\varphi}\right)\right| \leq L \sup _{t \in[0, T]}\left|X_{t}-X_{t}^{\varphi}\right| .
$$


Therefore decoupling properties of $X$ directly transfer to $\xi=g(X)$ and we may use the results of Section 4.7.1 of these notes.

(3) The spaces to describe the fractional smoothness of the terminal condition in 48, and 40] coincide with $\mathbb{B}_{p}^{\Phi}$ with $\Phi=\Phi_{r_{1}, \ldots, r_{L}}^{\left(\theta_{1}, \infty\right), \ldots,\left(\theta_{L}, \infty\right)}$ (in [48] with $L=1$ ) from Definition 4.18 below. So the present article generalizes results from $[40$ to the fully path-dependent case where no structural assumptions on the terminal condition nor the generator are imposed.

(4) In Section 6.3 we investigate the uniqueness and distributional properties of the $(Y, Z)$-processes of quadratic and sub-quadratic BSDEs that are not necessarily Markovian and that might have an unbounded terminal condition.

(5) In Section 6.4.4 we prove that regularity properties of a BSDE in terms of $\mathbb{B}_{p}^{\Phi}$ for the terminal condition $\xi$, and a similar one for the generator $f$, are transferred to the solution processes $(Y, Z)$ without structural assumptions on $(\xi, f)$. For the particular case described in item (3), this was partially done in the presence of a forward diffusion in 40 .

(6) Section 6.5 In the literature usually estimates of the form (8), that means estimates with the order $\frac{1}{2}$, are shown. This is due to Lipschitz or uniform $\mathbb{D}_{1,2}$ assumptions and appears in [52, Theorem 2.6, Corollary 2.7]. There regularity results for $Y$ and $Z$ of the form (8) for non-Markovian Lipschitz BSDEs were proven under Lipschitz assumptions for the generator and under assumptions on the Malliavin derivatives up to the second order of $\xi$ and $f$. In [52, Theorem 2.3] a condition $M^{2, q}$ is used to investigate the variation of the $Y$-process of the solution to a BSDE with a random linear generator. The structure of this BSDE yields to an explicit representation of the $Y$ process. The condition $M^{2, q}$ relates to our $\mathbb{B}_{p}^{\Phi_{2}}$ spaces via Theorem 4.22 Translated to our setting, the condition $M^{2, q}$ is a condition on the predictable projection of $\left(D_{t} \xi\right)_{t \in[0, T]}$, whereas our condition is a condition on $\left(D_{t} \xi\right)_{t \in[0, T]}$ itself - however, the condition in [52] is not a condition on $\xi$, but on $\xi \rho_{T}$, where $\rho_{T}$ is a stochastic exponential.

Parts of our contribution are: for the regularity of $Y$ we do not need to require assumptions on the differentiability of $\xi$ (for example), secondly we can also treat cases where we have rates in 8 weaker than $\frac{1}{2}$.

\subsection{Notation}

The spaces $\mathbb{R}^{n}$ are equipped with the Euclidean norm $|x|=\left(\sum_{j=1}^{n}\left|x_{j}\right|^{2}\right)^{\frac{1}{2}}$ so that $\left[\mathbb{R}^{n},|\cdot|\right]$ becomes a Hilbert space. Given a metric space $M$, we let $C(M)$ be the space of all continuous real valued mappings on $M$. For a probability space $(\Omega, \mathcal{F}, \mathbb{P})$ the space of all random variables $X: \Omega \rightarrow \mathbb{R}$, i.e. Borel measurable maps, is denoted by $\mathcal{L}_{0}(\Omega, \mathcal{F}, \mathbb{P})$ and equipped with the pseudo-metric

$$
d_{\Omega}(X, Y):=\int_{\Omega} \frac{|X(\omega)-Y(\omega)|}{1+|X(\omega)-Y(\omega)|} d \mathbb{P}(\omega)
$$

The space $\mathcal{L}_{p}(\Omega, \mathcal{F}, \mathbb{P}), p \in(0, \infty)$, consists of all random variables $X: \Omega \rightarrow \mathbb{R}$ on $(\Omega, \mathcal{F}, \mathbb{P})$ such that $\|X\|_{p}:=\left(\int_{\Omega}|X(\omega)|^{p} d \mathbb{P}(\omega)\right)^{1 / p}<\infty$. As usual, for $p=\infty$ we let $\|X\|_{\infty}:=\operatorname{esssup}_{\omega \in \Omega}|X(\omega)|<\infty$ which yields to the space $\mathcal{L}_{\infty}(\Omega, \mathcal{F}, \mathbb{P})$. By identifying two random variables $X$ and $Y$ on $(\Omega, \mathcal{F}, \mathbb{P})$ when $X=Y \mathbb{P}$-a.s., we 
obtain equivalence classes, denoted by $[X]$, the quasi-normed spaces $\left(L_{p}(\Omega, \mathcal{F}, \mathbb{P}), \|\right.$. $\left.\|_{p}\right)$ for $p \in(0, \infty]$, and the complete metric space $\left(L_{0}(\Omega, \mathcal{F}, \mathbb{P}), d_{\Omega}\right)$ with

$$
d_{\Omega}([X],[Y]):=d_{\Omega}(X, Y) .
$$

In Chapters 2 and 3 we carefully distinguish between equivalence classes and random variables, in the later chapters we follow the standard way to identify equivalence classes and random variables if there is no risk of confusion. For two real valued random variables $X$ and $Y$ or $\mathbb{R}^{n}$-valued random vectors $\left(X_{1}, \ldots, X_{n}\right)$ and $\left(Y_{1}, \ldots, Y_{n}\right)$ the notations $X \stackrel{d}{=} Y$ and $\left(X_{1}, \ldots, X_{n}\right) \stackrel{d}{=}\left(Y_{1}, \ldots, Y_{n}\right)$ mean equality in distribution. We shall use the Burkholder-Davis-Gundy inequalities for continuous local martingales [80, IV.4.1] with $\beta_{p} \geq 1$ as constant, i.e. given $p \in(0, \infty)$ and a continuous real-valued martingale $\left(M_{t}\right)_{t \in[0, T]}$ vanishing at zero, we have

$$
\frac{1}{\beta_{p}}\left\|\langle M\rangle_{T}^{\frac{1}{2}}\right\|_{p} \leq\left\|\sup _{t \in[0, T]}\left|M_{t}\right|\right\|_{p} \leq \beta_{p}\left\|\langle M\rangle_{T}^{\frac{1}{2}}\right\|_{p}
$$

where $\beta_{p} \geq 1$ is an absolute constant and $\langle M\rangle_{T}$ is the quadratic variation of $M$ at time $T$. We do not need the particular behaviour of the constants $\beta_{p}$, so that we use for the upper and lower bound the same constant. As conventions we use $0^{0}:=1$ and

$$
A \sim_{c} B \text { for } \quad \frac{1}{c} A \leq B \leq c A
$$

when $A, B \geq 0$ and $c \geq 1$. Finally, for a set $S$ and $A \subseteq S$ we define the indicator function $\chi_{A}: S \rightarrow \mathbb{R}$ as

$$
\chi_{A}(s):=\left\{\begin{array}{ll}
1 & : s \in A \\
0 & : s \notin A .
\end{array} .\right.
$$


7 Nov 2017 05:35:26 EST

Prob+Stat

Version 2 - Submitted to Memoirs of the AMS 


\section{CHAPTER 2}

\section{A General Factorization}

There exist several factorization techniques for random variables and stochastic processes that have the idea to factor a random variable or process through a canonical space that carries the typical information about the problem one is interested in. We will use this idea as an intermediate step to decouple in Chapter 4 the Wiener space and to generate anisotropic Besov spaces. For the Wiener space there are two natural choices as a canonical space: The function space of continuous functions that yields to the Wiener measure and the sequence space $\mathbb{R}^{\mathbb{N}}$ with $\mathbb{N}=\{0,1,2, \ldots\}$ that yields to an infinite product of standard Gaussian measures. We use the second approach as in $\mathbf{6 5}$ and $\mathbf{5 3}$, and extend this approach so that no particular distribution (like the Gaussian distribution) is needed and so that it includes the handling of the stochastic processes we work with later. The second approach is convenient for us because we need to consider, from the very beginning, only sequences of real valued random variables, and furthermore, it might be generalized to other canonical spaces than spaces of continuous functions.

Our factorization procedure yields to the operators $\mathcal{C}^{M}$ that are defined in two steps. First, we introduce the operators $\mathcal{C}$ acting on random variables, then we extend them to the operators $\mathcal{C}^{M}$ acting on random continuous functions defined on complete metric spaces, that are locally $\sigma$-compact.

\subsection{The operators $\mathcal{C}$ and $\mathcal{C}^{M}$}

We shall work with two probability spaces $\left(\Omega^{i}, \mathcal{F}^{i}, \mathbb{P}^{i}\right), i=0,1$, and random variables $\left(\xi_{k}^{i}\right)_{k \in I}, \xi_{k}^{i}: \Omega^{i} \rightarrow \mathbb{R}$, where $I=\{0, \ldots, K\}$ or $I=\{0,1,2, \ldots\}$, and assume that

(C1) $\mathcal{F}^{\xi, i}:=\sigma\left(\xi_{k}^{i}: k \in I\right)$,

(C2) $\mathcal{F}^{i}=\mathcal{F}^{\xi, i} \vee \mathcal{N}^{i}$, where $\mathcal{N}^{i}:=\left\{A^{i} \in \mathcal{F}^{i}: \mathbb{P}^{i}\left(A^{i}\right)=0\right\}$,

(C3) $\left(\xi_{k}^{0}\right)_{k \in I}$ and $\left(\xi_{k}^{1}\right)_{k \in I}$ have the same finite-dimensional distributions.

If we omit the superscript $i$ in $\Omega^{i}, \mathcal{F}^{i}, \mathbb{P}^{i},\left(\xi_{k}^{i}\right)_{k \in I}$, or $\mathcal{F}^{\xi, i}$, then we consider one of the both probability spaces together with the corresponding random variables and operators introduced later. Let $\mathcal{B}\left(\mathbb{R}^{I}\right)$ be the $\sigma$-algebra generated by the cylinder sets on $\mathbb{R}^{I}$, and let $\mathbb{P}^{c}$ be the law of the map

$$
J_{0}: \Omega \rightarrow \mathbb{R}^{I} \quad \text { with } \quad J_{0}(\omega):=\left(\xi_{k}(\omega)\right)_{k \in I} .
$$

By the assumption (C3) the measure $\mathbb{P}^{c}$ is the same for both cases $i=0,1$. Moreover, let us assume another probability space $(R, \mathcal{R}, \rho)$, and define

$$
J: R \times \Omega \rightarrow R \times \mathbb{R}^{I} \quad \text { with } \quad J(r, \omega):=\left(r, J_{0}(\omega)\right) .
$$

For the construction of the operator $\mathcal{C}$ we start with two lemmas: 
Lemma 2.1. For any $\mathcal{R} \otimes \mathcal{F}$-measurable random variable $X: R \times \Omega \rightarrow \mathbb{R}$ there is an $\mathcal{R} \otimes \mathcal{F}^{\xi}$-measurable random variable $X^{\xi}: R \times \Omega \rightarrow \mathbb{R}$ with $(\rho \otimes \mathbb{P})\left(X=X^{\xi}\right)=1$.

Proof. We show that the $\rho \otimes \mathbb{P}$-completion of $\mathcal{R} \otimes \mathcal{F}^{\xi}$ contains $\mathcal{R} \otimes \mathcal{F}$. It is sufficient to prove that $A \times B \in \overline{\mathcal{R} \otimes \mathcal{F} \xi}^{\rho \otimes \mathbb{P}}$ for $A \in \mathcal{R}$ and $B \in \mathcal{F}$. We find a $B^{\xi} \in \mathcal{F}^{\xi}$ such that $\mathbb{P}\left(B \Delta B^{\xi}\right)=0$. Hence $(A \times B) \Delta\left(A \times B^{\xi}\right)=A \times\left(B \Delta B^{\xi}\right)$ is of $\rho \otimes \mathbb{P}$-measure zero. Because of $A \times B^{\xi} \in \mathcal{R} \otimes \mathcal{F}^{\xi}$ we can conclude the proof.

Lemma 2.2. The following assertions hold true:

(1) For each $\mathcal{R} \otimes \mathcal{F}^{\xi}$-measurable random variable $X: R \times \Omega \rightarrow \mathbb{R}$ there exists a random variable $\widehat{X}: R \times \mathbb{R}^{I} \rightarrow \mathbb{R}$ such that

$$
X:(R \times \Omega) \stackrel{J}{\rightarrow}\left(R \times \mathbb{R}^{I}\right) \stackrel{\widehat{X}}{\rightarrow} \mathbb{R} .
$$

(2) For $\mathcal{R} \otimes \mathcal{F}^{\xi, 0}$-measurable random variables $X, X^{\prime}: R \times \Omega^{0} \rightarrow \mathbb{R}$ with $(\rho \otimes$ $\left.\mathbb{P}^{0}\right)\left(X=X^{\prime}\right)=1$ one has $\left(\rho \otimes \mathbb{P}^{1}\right)\left(\widehat{X} \circ J^{1}=\widehat{X}^{\prime} \circ J^{1}\right)=1$ where the factorizations $X=\widehat{X} \circ J^{0}$ and $X^{\prime}=\widehat{X^{\prime}} \circ J^{0}$ are obtained by part (1).

Proof. (1) The map $J$ generates the $\sigma$-algebra $\mathcal{R} \otimes \mathcal{F}^{\xi}$. Hence we apply the functional representation from the Factorization Lemma [7, p. 62] and (1) follows. (2) The assumption implies by a change of variables $\left(\rho \otimes \mathbb{P}^{c}\right)\left(\widehat{X}=\widehat{X^{\prime}}\right)=1$, and by another change of variables the conclusion of assertion (2).

The above lemma enables us to introduce the operator $\mathcal{C}$ that maps an equivalence class $[X]$ from $L_{0}\left(R \times \Omega^{0}\right)$ to the equivalence class $\left[\widehat{X} \circ J^{1}\right]$ in $L_{0}\left(R \times \Omega^{1}\right)$ so that $[X]$ and $\left[\widehat{X} \circ J^{1}\right]$ have the same law.

\section{DEFINITION 2.3.}

(1) We define the map $\mathcal{C}: L_{0}\left(R \times \Omega^{0}\right) \rightarrow L_{0}\left(R \times \Omega^{1}\right)$ by

$$
\mathcal{C}(X)=\mathcal{C}([X]):=\left[\widehat{X} \circ J^{1}\right]
$$

where $X \in[X]$ is an $\mathcal{R} \otimes \mathcal{F}^{\xi, 0}$-measurable representative of $[X]$.

(2) We define the map $\mathcal{C}_{0}: L_{0}\left(\Omega^{0}\right) \rightarrow L_{0}\left(\Omega^{1}\right)$ by

$$
\mathcal{C}_{0}(X)=\mathcal{C}_{0}([X]):=\left[\widehat{X} \circ J_{0}^{1}\right],
$$

where $X \in[X]$ is an $\mathcal{F}^{\xi, 0}$-measurable representative of $[X]$.

Part (2) of Definition 2.3 corresponds to the case where $R=\left\{r_{0}\right\}$ is a singleton. We gave a separate definition since $\mathcal{C}_{0}$ will play a particular role later on. Basic properties of $\mathcal{C}_{0}$ and $\mathcal{C}$ are summarized in Proposition 2.5 below. For its formulation we need a class of functionals $\Phi: \mathcal{L}_{0}(R) \times \cdots \times \mathcal{L}_{0}(R) \rightarrow \mathbb{R}$ that, for example in the case $(R, \mathcal{R}, \rho)=([0,1], \mathcal{B}([0,1]), \lambda)$ with $\lambda$ being the Lebesgue measure, excludes Dirac functionals $\Phi(f):=f\left(r_{0}\right)$, where $r_{0} \in[0,1]$ is fixed.

Definition 2.4. A functional $\Phi:\left(\mathcal{L}_{0}(R)\right)^{n} \rightarrow \mathbb{R}$ is called consistent provided that for all probability spaces $(A, \mathcal{A}, \mathbb{Q})$ and jointly measurable $X_{1}, \ldots, X_{n}: R \times A \rightarrow \mathbb{R}$ the map $F_{X}: A \rightarrow \mathbb{R}$ with

$$
F_{X}(\omega)=\Phi\left(X_{1}(\cdot, \omega), \ldots, X_{n}(\cdot, \omega)\right)
$$

is measurable and $\mathbb{Q}\left(F_{X}=F_{X^{\prime}}\right)=1$ if $(\rho \otimes \mathbb{Q})\left(X_{i} \neq X_{i}^{\prime}\right)=0$ for $i=1, \ldots, n$. 
Proposition 2.5. For $X, X_{1}, \ldots, X_{n} \in \mathcal{L}_{0}\left(R \times \Omega^{0}\right)$ and $Y_{i} \in \mathcal{C}\left(X_{i}\right), i=1, \ldots, n$, the following holds true:

(1) $\xi_{k}^{1} \in \mathcal{C}_{0}\left(\xi_{k}^{0}\right)$ for $k \in I$.

(2) $\mathcal{C}$ is a linear isometry and bijection.

(3) $\left(Y_{1}, \ldots, Y_{n}\right) \stackrel{d}{=}\left(X_{1}, \ldots, X_{n}\right)$.

(4) For a Borel function $g: \mathbb{R}^{n} \rightarrow \mathbb{R}$ one has

$$
g\left(Y_{1}, \ldots, Y_{n}\right) \in \mathcal{C}\left(g\left(X_{1}, \ldots, X_{n}\right)\right) .
$$

(5) If $\Phi: \mathcal{L}_{0}(R) \times \cdots \times \mathcal{L}_{0}(R) \rightarrow \mathbb{R}$ is consistent, then

$$
\Phi\left(Y_{1}, \ldots, Y_{n}\right) \in \mathcal{C}_{0}\left(\Phi\left(X_{1}, \ldots, X_{n}\right)\right) .
$$

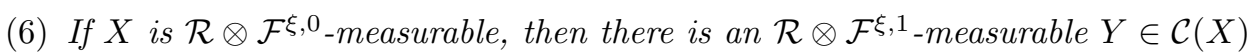
such that for all $r \in R$ one has

$$
Y(r, \cdot) \in \mathcal{C}_{0}(X(r, \cdot)) .
$$

(7) For $Y \in \mathcal{L}_{0}\left(R \times \Omega^{1}\right)$ one has $Y \in \mathcal{C}(X)$ if and only if there is a null-set $\mathcal{N} \subseteq R$ such that for all $r \in R \backslash \mathcal{N}$ one has

$$
Y(r, \cdot) \in \mathcal{C}_{0}(X(r, \cdot)) .
$$

Proof. (1) follows from the definition of $\mathcal{C}_{0}$.

(2) Linearity: Let $a, b \in \mathbb{R}$ and $X, Y \in \mathcal{L}_{0}\left(R \times \Omega^{0}\right)$, and take $\mathcal{R} \otimes \mathcal{F}^{\xi, 0}$-measurable representatives $X^{\xi} \in[X]$ and $Y^{\xi} \in[Y]$. Then $a X^{\xi}+b Y^{\xi} \in a[X]+b[Y]$. From Lemma 2.2 we get that

$$
X^{\xi}(\eta)=\widehat{X^{\xi}} \circ J^{0}(\eta) \quad \text { and } \quad Y^{\xi}(\eta)=\widehat{Y^{\xi}} \circ J^{0}(\eta),
$$

for all $\eta \in R \times \Omega^{0}$. Defining point-wise

$$
T:=a \widehat{X^{\xi}}+b \widehat{Y^{\xi}},
$$

we get that $T: R \times \mathbb{R}^{I} \rightarrow \mathbb{R}$ is measurable and

$$
T\left(J^{0}(\eta)\right)=a X^{\xi}(\eta)+b Y^{\xi}(\eta) \text { for all } \eta \in R \times \Omega^{0}
$$

so that $T\left(J^{0}\right) \in\left[a X^{\xi}+b Y^{\xi}\right]$. By definition of $\mathcal{C}$,

$$
T\left(J^{1}\right)=a \widehat{X^{\xi}} \circ J^{1}+b \widehat{Y^{\xi}} \circ J^{1} \in \mathcal{C}(a X+b Y),
$$

but is also an element of $a \mathcal{C}(X)+b \mathcal{C}(Y)$.

Isometry: Because the laws of $J^{0}$ and $J^{1}$ coincide, it follows that $X$ and the representatives of $\mathcal{C}(X)$ have the same distribution. As $d\left(X, X^{\prime}\right)=d\left(X-X^{\prime}, 0\right)$ the property that $\mathcal{C}$ is an isometry follows immediately.

BiJection: Since $\mathcal{C}$ is an isometry, it is an injection. Now let $Y \in \mathcal{L}_{0}\left(R \times \Omega^{1}\right)$ and take $Y^{\xi}$ to be an $\mathcal{R} \otimes \mathcal{F}^{\xi, 1}$-measurable representative of $[Y]$. Then there is a measurable $\widehat{Y^{\xi}}: R \times \mathbb{R}^{I} \rightarrow \mathbb{R}$ such that

$$
Y^{\xi}(\eta)=\widehat{Y^{\xi}} \circ J^{1}(\eta) \text { for all } \eta \in R \times \Omega^{1} .
$$

Now $\eta \mapsto \widehat{Y^{\xi}} \circ J^{0}(\eta)$ is $\mathcal{R} \otimes \mathcal{F}^{\xi, 0}$-measurable and

$$
\mathcal{C}\left(\left[\widehat{Y}^{\xi} \circ J^{0}\right]\right)=[Y]
$$


(3) The characteristic functions of $\left(X_{1}, \ldots, X_{n}\right)$ and $\left(Y_{1}, \ldots, Y_{n}\right)$ coincide, because for all $\left(t_{1}, \ldots, t_{n}\right) \in \mathbb{R}^{n}$ and $Y_{k} \in \mathcal{C}\left(X_{k}\right)$ we have

$$
\int_{R \times \Omega^{1}} e^{i \sum_{k=1}^{n} t_{k} Y_{k}} d\left(\rho \otimes \mathbb{P}^{1}\right)=\int_{R \times \Omega^{0}} e^{i \sum_{k=1}^{n} t_{k} X_{k}} d\left(\rho \otimes \mathbb{P}^{0}\right)
$$

where we used (2) and that $\mathcal{C}$ keeps the distribution invariant.

(4) We choose $X_{1}^{\xi}, \ldots, X_{n}^{\xi}$ to be $\mathcal{R} \otimes \mathcal{F}^{\xi, 0}$-measurable representatives of the classes $\left[X_{1}\right], \ldots,\left[X_{n}\right]$, so that

$$
X_{i}^{\xi}(\eta)=\widehat{X_{i}^{\xi}} \circ J^{0}(\eta)
$$

for $i=1, \ldots, n$ and all $\eta \in R \times \Omega^{0}$. Next we define the measurable functional $T_{Z}: R \times \mathbb{R}^{I} \rightarrow \mathbb{R}$ as

$$
T_{Z}(\zeta):=g\left(\widehat{X_{1}^{\xi}}(\zeta), \ldots, \widehat{X_{n}^{\xi}}(\zeta)\right)
$$

so that $T_{Z} \circ J^{0}=g\left(X_{1}^{\xi}, \ldots, X_{n}^{\xi}\right)$. By definition of $\mathcal{C}$ we get that

$$
\mathcal{C}\left(g\left(X_{1}^{\xi}, \ldots, X_{n}^{\xi}\right)\right)=\left[T_{Z} \circ J^{1}\right] .
$$

On the other side, by definition of $T_{Z}$ we have that

$$
T_{Z} \circ J^{1}=g\left(\widehat{X_{1}^{\xi}} \circ J^{1}, \ldots, \widehat{X_{n}^{\xi}} \circ J^{1}\right),
$$

which is $\rho \otimes \mathbb{P}^{1}$-a.s. the same as $g\left(Y_{1}, \ldots, Y_{n}\right)$, where $Y_{i} \in \mathcal{C}\left(X_{i}\right)$. This concludes the proof.

(5) We choose $\mathcal{R} \otimes \mathcal{F}^{\xi, 0}$-measurable representatives $X_{i}^{\xi} \in\left[X_{i}\right]$, define $Y_{i}^{\xi}:=\widehat{X_{i}^{\xi}} \circ J^{1}$, and get

$$
\begin{aligned}
& F_{Y^{\xi}}\left(\omega^{1}\right)=\Phi\left(\widehat{X_{1}^{\xi}}\left(\cdot, J_{0}^{1}\left(\omega^{1}\right)\right), \ldots, \widehat{X_{N}^{\xi}}\left(\cdot, J_{0}^{1}\left(\omega^{1}\right)\right)\right), \\
& F_{X^{\xi}}\left(\omega^{0}\right)=\Phi\left(\widehat{X_{1}^{\xi}}\left(\cdot, J_{0}^{0}\left(\omega^{0}\right)\right), \ldots, \widehat{X_{N}^{\xi}}\left(\cdot, J_{0}^{0}\left(\omega^{0}\right)\right)\right) .
\end{aligned}
$$

Defining $\Psi: \mathbb{R}^{I} \rightarrow \mathbb{R}$ by $\Psi(\zeta):=\Phi\left(\widehat{X_{1}^{\xi}}(\cdot, \zeta), \ldots, \widehat{X_{N}^{\xi}}(\cdot, \zeta)\right)$, our assumptions yields to a measurable map and $F_{X \xi}=\Psi \circ J_{0}^{0}$ and $F_{Y^{\xi}}=\Psi \circ J_{0}^{1}$. Consequently, $F_{Y^{\xi}} \in$ $\mathcal{C}_{0}\left(F_{X \xi}\right)$. Finally, our assumption yields that $F_{X \xi}$ and $F_{X}$ belong to the same equivalence class, and $F_{Y \xi}$ and $F_{Y}$ belong to the same equivalence class, so that the proof is complete.

(6) We have that $X=\widehat{X} \circ J^{0}$ for some $\widehat{X}$, which implies $X(r)=\widehat{X}\left(r, J_{0}^{0}\right)$ for all $r \in R$, and define $Y:=\widehat{X} \circ J^{1}$. By construction this implies that $Y(r)=\widehat{X}\left(r, J_{0}^{1}\right)$ for all $r \in R$.

(7) Choose $X^{\xi} \in[X]$ to be $\mathcal{R} \otimes \mathcal{F}^{\xi, 0}$-measurable and $Y^{\xi}:=\widehat{X^{\xi}} \circ J^{1}$ so that

$$
Y^{\xi} \in \mathcal{C}(X) \quad \text { and } \quad Y^{\xi}(r) \in \mathcal{C}_{0}\left(X^{\xi}(r)\right)
$$

for all $r \in R$. Moreover, $\mathbb{P}^{0}\left(X^{\xi}(r)=X(r)\right)=1$ for $r \in R \backslash \mathcal{N}^{\prime}$ where $\mathcal{N}^{\prime} \subseteq R$ is a null-set, so that

$$
\mathcal{C}_{0}\left(X^{\xi}(r)\right)=\mathcal{C}_{0}(X(r))
$$

for all $r \in R \backslash \mathcal{N}^{\prime}$. Hence, $Y^{\xi}(r) \in \mathcal{C}_{0}(X(r))$ for all $r \in R \backslash \mathcal{N}^{\prime}$. The claim now follows from the fact, that for $Y \in \mathcal{L}_{0}\left(R \times \Omega^{1}\right)$ we have that $Y \in \mathcal{C}(X)$ if and only if $\mathbb{P}^{1}\left(Y(r)=Y^{\xi}(r)\right)=1$ for all $r \in R \backslash \mathcal{N}^{\prime \prime}$, where $\mathcal{N}^{\prime \prime} \subseteq R$ is a null-set. 
We extend our definition of $\mathcal{C}$ to decouple later random generators of BSDEs. Let $M$ be a complete metric space that is locally $\sigma$-compact, i.e. there exist compact subsets $\emptyset \neq K_{1} \subseteq K_{2} \subseteq \ldots$, such that $\overline{\stackrel{\circ}{K}}_{n}=K_{n}$ and $M=\cup_{n=1}^{\infty} \stackrel{\circ}{K}_{n}$. For the following we recall that $C(M)$ is the space of continuous $\mathbb{R}$-valued functions on $M$.

Definition 2.6. Given a measurable space $(A, \mathcal{A})$, we let $f \in \mathcal{L}_{0}(A ; C(M))$ if and only if $f: A \times M \rightarrow \mathbb{R}$ is a Carathéodory function, i.e. $f$ satisfies that

(a) $\alpha \rightarrow f(\alpha, x)$ is measurable for all $x \in M$,

(b) $x \rightarrow f(\alpha, x)$ is continuous for all $\alpha \in A$.

If $(A, \mathcal{A})$ is equipped with a probability measure $\mathbb{Q}$, then the space $L_{0}(A ; C(M))$ is the space of equivalence classes with $f \sim g$ if $\mathbb{Q}(f(x)=g(x), x \in M)=1$.

REMARK 2.7. Equivalently, a Carathéodory function is a measurable function $f$ : $A \rightarrow C(M)$, when $C(M)$ is equipped with the smallest $\sigma$-algebra $\mathcal{B}(C(M))$ such that for all $x \in M$ the maps $\delta_{x}: C(M) \rightarrow \mathbb{R}$ with $\delta_{x}(f):=f(x)$ are Borelmeasurable.

The next lemma extends the operator $\mathcal{C}$ to $C(M)$-valued random variables.

Lemma 2.8. For $f \in \mathcal{L}_{0}\left(R \times \Omega^{0} ; C(M)\right)$ there is a $g \in \mathcal{L}_{0}\left(R \times \Omega^{1} ; C(M)\right)$ with $g(x) \in \mathcal{C}(f(x))$ for all $x \in M$. If $g_{1}$ and $g_{2}$ satisfy this property, then $g_{1}=g_{2}$ $\left(\rho \otimes \mathbb{P}^{1}\right)$-a.s.

Proof. Proposition 2.5 implies that $(f(x))_{x \in M}$ and $(h(x))_{x \in M}$ have the same finite-dimensional distributions for $h(x) \in \mathcal{C}(f(x))$, so that the result follows from Proposition A.1.

Now we are ready to introduce the extension $\mathcal{C}^{M}$ of $\mathcal{C}$ that maps equivalence classes from $L_{0}\left(R \times \Omega^{0} ; C(M)\right)$ to $L_{0}\left(R \times \Omega^{1} ; C(M)\right)$ while keeping the distributional properties of the equivalence classes.

Definition 2.9. We let

$$
\mathcal{C}^{M}: L_{0}\left(R \times \Omega^{0} ; C(M)\right) \rightarrow L_{0}\left(R \times \Omega^{1} ; C(M)\right)
$$

such that $\mathcal{C}^{M}([f])$ is the unique equivalence-class whose representatives $g$ satisfy $g(x) \in \mathcal{C}(f(x))$ for all $x \in M$. Moreover, we define $\mathcal{C}^{M}(f):=\mathcal{C}^{M}([f])$ for $f \in \mathcal{L}_{0}\left(R \times \Omega^{0} ; C(M)\right)$.

\subsection{The operators $\mathcal{C}$ and $\mathcal{C}^{M}$ for stochastic processes}

In this section we specialize to stochastic processes $X:[0, T] \times \Omega \rightarrow \mathbb{R}$, where $T \in(0, \infty)$ is fixed. This means, that we complement some results from Section 2.1 in the case $(R, \mathcal{R}, \rho)=([0, T], \mathcal{B}([0, T]), \lambda / T)$ where $\lambda$ is the Lebesgue measure. Here we distinguish more clearly between the operators $\mathcal{C}$ and $\mathcal{C}_{0}$ from Definition 2.3 . We will use the following notation:

\begin{tabular}{|ll|ll|}
\hline$\Omega_{0}:=\quad \Omega$ & $\Omega_{T}:=[0, T] \times \Omega$ \\
$\Sigma_{0}^{\xi}:=\mathcal{F} \xi$ & $\Sigma_{T}^{\xi}:=\mathcal{B}([0, T]) \otimes \mathcal{F} \xi$ \\
$\Sigma_{0}:=\mathcal{F}$ & $\Sigma_{T}:=\mathcal{B}([0, T]) \otimes \mathcal{F}$ \\
$\mathbb{P}_{0}:=\mathbb{P}$ & $\mathbb{P}_{T}:=(\lambda \times \mathbb{P}) / T$ \\
$\mathcal{C}_{0}$ from Definition 2.3 & $\mathcal{C}_{T}:=\mathcal{C}$ \\
\hline
\end{tabular}


REMARK 2.10. One might also consider the infinite time interval $[0, \infty)$ by the choice $(R, \mathcal{R}, \rho)=([0, \infty), \mathcal{B}([0, \infty)), \mu)$, where (for example) $\mu$ is a probability measure with the same null-sets as the Lebesgue measure.

First we show how continuity and measurability properties are transferred by the operators $\mathcal{C}_{0}$ and $\mathcal{C}_{T}$. Here we use the following convention:

Convention 2.11. Let $S \in\{0, T\}$ and assume a sub- $\sigma$-algebra $\mathcal{G}_{S} \subseteq \Sigma_{S}$. We will interpret $L_{0}\left(\Omega_{S}, \mathcal{G}_{S}, \mathbb{P}_{S}\right)$ as the space of equivalence classes $[X] \in L_{0}\left(\Omega_{S}, \Sigma_{S}, \mathbb{P}_{S}\right)$ that contain a $\mathcal{G}_{S}$-measurable representative. Similarly, $L_{0}\left(\Omega_{S}, \mathcal{G}_{S}, \mathbb{P}_{S} ; C(M)\right)$ is the space of equivalence classes $[X] \in L_{0}\left(\Omega_{S}, \Sigma_{S}, \mathbb{P}_{S} ; C(M)\right)$ that contain a $\left(\mathcal{G}_{S}, \mathcal{B}(C(M))\right)$-measurable representative.

Proposition 2.12. For $i=0,1$ assume right-continuous filtrations $\mathbb{G}^{i}=\left(\mathcal{G}_{t}^{i}\right)_{t \in[0, T]}$ with $\mathcal{G}_{t}^{i} \subseteq \mathcal{F}^{i}$ such that $\mathcal{G}_{0}^{i}$ contains all null-sets of $\mathcal{F}^{i}$ and

$$
\mathcal{C}_{0}\left(L_{0}\left(\Omega^{0}, \mathcal{G}_{t}^{0}\right)\right) \subseteq L_{0}\left(\Omega^{1}, \mathcal{G}_{t}^{1}\right) \quad \text { for all } \quad t \in[0, T]
$$

Then the following assertions are true:

(1) If $X$ is path-wise continuous and $\mathbb{G}^{0}$-adapted, then there exists a path-wise continuous $\mathbb{G}^{1}$-adapted process $Y \in \mathcal{L}_{0}\left(\Omega_{T}^{1}\right)$ with

$$
Y(t) \in \mathcal{C}_{0}(X(t)) \quad \text { for all } t \in[0, T] .
$$

(2) One has $\mathcal{C}_{T}\left(L_{0}\left(\Omega_{T}^{0}, \mathcal{P}_{T}^{0}\right)\right) \subseteq L_{0}\left(\Omega_{T}^{1}, \mathcal{P}_{T}^{1}\right)$, where $\mathcal{P}_{T}^{i}$ are the predictable $\sigma$-algebras generated by the $\mathbb{G}^{i}$-adapted processes with paths that are left-continuous and have limits from the right.

Proof. (1) Taking $\beta(t) \in \mathcal{C}_{0}(X(t))$ to be $\mathcal{G}_{t}^{1}$-measurable, Proposition 2.5 (3) implies that $(\beta(t))_{t \in[0, T]}$ and $(X(t))_{t \in[0, T]}$ have the same finite-dimensional distributions. For $M=[0, T]$ we can use in the proof of Proposition A.1 the sets $K_{1}=K_{2}=\cdots=M$ and $D_{0}=A=[0, T] \cap \mathbb{Q}$. Furthermore, in the proof of Proposition A.1 we note that $Y(t)$ is defined as the a.s.-limit of $\beta_{t_{n}}$, where we may take now $t_{n} \uparrow t$. By our assumption $\beta_{t_{n}} \in \mathcal{L}_{0}\left(\Omega^{1}, \mathcal{G}_{t_{n}}^{1}\right)$, so that $Y(t)$ is $\mathcal{G}_{t}^{1}$-measurable. The facts that $(Y(t))_{t \in[0, T]}$ is continuous and a modification of $\left(\beta_{t}\right)_{t \in[0, T]}$ were proven in Proposition A.1.

(2) Applying [58, p. 133, step (b) of the proof of Lemma 2.4] we can approximate any predictable process $X \in \mathcal{L}_{0}\left(\Omega_{T}^{0}, \mathcal{P}_{T}^{0}\right)$ by a sequence of continuous adapted processes $X^{n} \in \mathcal{L}_{0}\left(\Omega_{T}^{0}, \mathcal{P}_{T}^{0}\right)$ with $d_{T}^{0}\left(X^{n}, X\right) \rightarrow_{n} 0$ (first we approximate $X$ by bounded processes by truncation, then we use [58]). Applying part (1), we find continuous adapted processes $Y^{n}$ such that $\lim _{n} d_{T}^{1}\left(Y^{n}, Y\right) \rightarrow_{n} 0$ for $Y \in \mathcal{C}_{T}(X)$. Because of $Y^{n} \in \mathcal{L}_{0}\left(\Omega_{T}^{1}, \mathcal{P}_{T}^{1}\right)$ we can choose $Y \in \mathcal{L}_{0}\left(\Omega_{T}^{1}, \mathcal{P}_{T}^{1}\right)$ as well.

The next proposition is needed later for technical reasons:

Proposition 2.13. The following assertions hold true:

(1) For $M:=\mathbb{R}^{d}, f \in \mathcal{L}_{0}\left(\Omega_{T}^{0} ; C(M)\right), X_{1}, \ldots, X_{d} \in \mathcal{L}_{0}\left(\Omega_{T}^{0}\right), g \in \mathcal{C}_{T}^{M}(f)$, and $Y_{i} \in \mathcal{C}_{T}\left(X_{i}\right), i=1, \ldots, d$, one has that

$$
g(\cdot, Y(\cdot)) \in \mathcal{C}_{T}(f(\cdot, X(\cdot)))
$$


(2) Let $S \in\{0, T\}$ and let $M$ be a complete metric space that is locally $\sigma$-compact. If one has that $\mathcal{C}_{S}\left(L_{0}\left(\Omega_{S}^{0}, \mathcal{G}_{S}^{0}\right)\right) \subseteq L_{0}\left(\Omega_{S}^{1}, \mathcal{G}_{S}^{1}\right)$ for $\sigma$-algebras $\mathcal{G}_{S}^{i} \subseteq \Sigma_{S}^{i}$, then

$$
\mathcal{C}_{S}^{M}\left(L_{0}\left(\Omega_{S}^{0}, \mathcal{G}_{S}^{0} ; C(M)\right)\right) \subseteq L_{0}\left(\Omega_{S}^{1}, \mathcal{G}_{S}^{1} ; C(M)\right) .
$$

Proof. (1a) First note that Lemma A.2 implies

$$
(f(t, X(t)))_{t \in[0, T]} \in \mathcal{L}_{0}\left(\Omega_{T}^{0}\right) \quad \text { and } \quad(g(t, Y(t)))_{t \in[0, T]} \in \mathcal{L}_{0}\left(\Omega_{T}^{1}\right) .
$$

(1b) Define for $i=1, \ldots, d, n \geq 1, a_{k} \in \mathbb{R}$ and a Borel-measurable partition $\bigcup_{k=0}^{n} B_{k}=\mathbb{R}$ with $B_{k} \neq \emptyset$ the processes

$$
\begin{aligned}
& A_{i}(t):=\sum_{k=0}^{n} a_{k} 1_{B_{k}}\left(X_{i}(t)\right) \quad \text { and } \quad A(t)=\left(A_{1}(t), \ldots, A_{d}(t)\right), \\
& D_{i}(t):=\sum_{k=0}^{n} a_{k} 1_{B_{k}}\left(Y_{i}(t)\right) \quad \text { and } \quad D(t)=\left(D_{1}(t), \ldots, D_{d}(t)\right) .
\end{aligned}
$$

By Proposition 2.5 we conclude

$$
\begin{aligned}
& \mathcal{C}_{T}\left((f(t, A(t)))_{t \in[0, T]}\right) \\
= & \sum_{n_{1}, \ldots, n_{d}=0}^{n} \mathcal{C}_{T}\left(\left(f\left(t, a_{n_{1}}, \ldots, a_{n_{d}}\right) 1_{B_{n_{1}} \times \cdots \times B_{n_{d}}}\left(X_{1}(t), \ldots, X_{d}(t)\right)\right)_{t \in[0, T]}\right) \\
= & \sum_{n_{1}, \ldots, n_{d}=0}^{n} \mathcal{C}_{T}\left(\left(f\left(t, a_{n_{1}}, \ldots, a_{n_{d}}\right)\right)_{t \in[0, T]}\right) \\
& \mathcal{C}_{T}\left(\left(1_{B_{n_{1}} \times \cdots \times B_{n_{d}}}\left(X_{1}(t), \ldots, X_{d}(t)\right)\right)_{t \in[0, T]}\right) \\
\ni & \sum_{n_{1}, \ldots, n_{d}=0}^{n}\left(g\left(t, a_{n_{1}}, \ldots, a_{n_{d}}\right)\right)_{t \in[0, T]}\left(1_{B_{n_{1}} \times \cdots \times B_{n_{d}}}\left(Y_{1}(t), \ldots, Y_{d}(t)\right)\right)_{t \in[0, T]} \\
= & (g(t, D(t)))_{t \in[0, T]},
\end{aligned}
$$

where the multiplication of equivalence classes is defined as usual.

(1c) For $L_{n}(x):=\sum_{k=-4^{n}}^{4^{n}-1} \frac{k}{2^{n}} 1_{\left[\frac{k}{2^{n}}, \frac{k+1}{2^{n}}\right)}(x)$ with $x \in \mathbb{R}$ we let

$$
A_{i}^{n}(t):=L_{n}\left(X_{i}(t)\right) \quad \text { and } \quad D_{i}^{n}(t):=L_{n}\left(Y_{i}(t)\right)
$$

so that $d_{T}^{0}\left(A_{i}^{n}, X_{i}\right) \rightarrow_{n} 0$ for $i=1, \ldots, d$. Proposition 2.5 yields $D_{i}^{n} \in \mathcal{C}_{T}\left(A_{i}^{n}\right)$ and $d_{T}^{1}\left(D_{i}^{n}, Y_{i}\right)=d_{T}^{1}\left(\mathcal{C}_{T}\left(A_{i}^{n}\right), \mathcal{C}_{T}\left(X_{i}\right)\right) \rightarrow_{n} 0$. Because of step (b) and because $\mathcal{C}_{T}$ is an isometry, we obtain the estimates

$$
\begin{aligned}
& d_{T}^{1}\left(\mathcal{C}_{T}\left((f(t, X(t)))_{t \in[0, T]}\right),\left[(g(t, Y(t)))_{t \in[0, T]}\right]\right) \\
\leq \quad & d_{T}^{1}\left(\mathcal{C}_{T}\left((f(t, X(t)))_{t \in[0, T]}\right), \mathcal{C}_{T}\left(\left(f\left(t, A^{n}(t)\right)\right)_{t \in[0, T]}\right)\right) \\
& +d_{T}^{1}\left(\mathcal{C}_{T}\left(\left(f\left(t, A^{n}(t)\right)\right)_{t \in[0, T]}\right),\left[\left(g\left(t, D^{n}(t)\right)\right)_{t \in[0, T]}\right]\right) \\
& +d_{T}^{1}\left(\left[\left(g\left(t, D^{n}(t)\right)\right)_{t \in[0, T]}\right],\left[(g(t, Y(t)))_{t \in[0, T]}\right]\right) \\
= & d_{T}^{0}\left((f(t, X(t)))_{t \in[0, T]},\left(f\left(t, A^{n}(t)\right)\right)_{t \in[0, T]}\right) \\
& +d_{T}^{1}\left(\left(g\left(t, D^{n}(t)\right)\right)_{t \in[0, T]},(g(t, Y(t)))_{t \in[0, T]}\right) .
\end{aligned}
$$

Because $f\left(t, A^{n}(t)\right) \rightarrow_{n} f(t, X(t))$ for all $(t, \omega) \in \Omega_{T}^{0}$, we have that

$$
d_{T}^{0}\left((f(t, X(t)))_{t \in[0, T]},\left(f\left(t, A^{n}(t)\right)\right)_{t \in[0, T]}\right) \rightarrow_{n} 0 .
$$


For the last expression we use that $D_{i}^{n} \rightarrow_{n} Y_{i}$ in probability implies the convergence $\left(g\left(t, D^{n}(t)\right)\right)_{t \in[0, T]} \rightarrow_{n}(g(t, Y(t)))_{t \in[0, T]}$ in probability as well.

(2) From Proposition A.1 it follows that the equivalence-class $\mathcal{C}_{S}^{M}(f)$ contains a $\left(\mathcal{G}_{S}^{1}, \mathcal{B}(C(M))\right)$-measurable representative.

We conclude with some comments on part (5) of Proposition 2.5

\section{REMARK 2.14 .}

(1) Let $L_{0}([0, T])$ be equipped with the Borel $\sigma$-algebra, obtained by the metric of type 110 from Section 1.3 and assume a $\otimes_{1}^{n} \mathcal{B}\left(L_{0}([0, T])\right)$-measurable $\Psi$ : $\left(L_{0}([0, T])\right)^{n} \rightarrow \mathbb{R}$ such that

$$
\Phi\left(f_{1}, \ldots, f_{n}\right)=\Psi\left(\left[f_{1}\right], \ldots,\left[f_{n}\right]\right) \text { for } f_{1}, \ldots, f_{n} \in \mathcal{L}_{0}([0, T]) .
$$

Then $\Phi$ is consistent.

In fact, the space $L_{0}([0, T])$ is separable so that its Borel $\sigma$-algebra is generated by the open balls. We equip $\mathcal{L}_{0}([0, T])$ with the smallest $\sigma$-algebra $\mathcal{B}\left(\mathcal{L}_{0}([0, T])\right)$ such that $q: \mathcal{L}_{0}([0, T]) \rightarrow L_{0}([0, T])$ with $q(f):=[f]$ is measurable. A measurable process $X:[0, T] \times A \rightarrow \mathbb{R}$ generates a canonical map $\hat{X}: A \rightarrow \mathcal{L}_{0}([0, T])$ that is measurable because

$$
\left\{\omega \in A: \int_{0}^{T} \frac{|X(t, \omega)-f(t)|}{1+|X(t, \omega)-f(t)|} d t<\varepsilon\right\} \in \mathcal{A}
$$

for all $\varepsilon>0$ and $f \in \mathcal{L}_{0}([0, T])$. Hence we can finish the proof as the composition of two measurable maps is measurable.

(2) For a measurable $\phi:[0, T] \times \mathbb{R}^{n} \rightarrow \mathbb{R}$ and $g=\left(g_{1}, \ldots, g_{n}\right) \in\left(\mathcal{L}_{0}([0, T])\right)^{n}$ we obtain a consistent functional by

$$
\Phi(g):=\int_{0}^{T} \phi(t, g(t)) \chi_{\left\{\int_{0}^{T}|\phi(t, g(t))| d t<\infty\right\}} d t .
$$

Applying Proposition 2.5(5) to the function $\phi(t, x):=|x|^{p} \wedge L$ with $L, p \in(0, \infty)$ and $x \in \mathbb{R}^{n}$, we get that

$$
\int_{0}^{T}\left(|Y(t)|^{p} \wedge L\right) d t \in \mathcal{C}_{0}\left(\int_{0}^{T}\left(|X(t)|^{p} \wedge L\right) d t\right)
$$

for $X(t)=\left(X_{1}(t), \ldots, X_{n}(t)\right)$ and $Y(t)=\left(Y_{1}(t), \ldots, Y_{n}(t)\right)$, where $X_{i} \in \mathcal{L}_{0}\left(\Omega_{T}^{0}\right)$ and $Y_{i} \in \mathcal{C}_{T}\left(X_{i}\right)$ for $i=1, \ldots, n$. Assuming that $\int_{0}^{T}|X(t, \omega)|^{p} d t<\infty$ for all $\omega \in \Omega^{0}$, we have

$$
\lim _{N \rightarrow \infty}\left(\int_{0}^{T}\left(|X(t, \omega)|^{p} \wedge N\right) d t\right)=\int_{0}^{T}|X(t, \omega)|^{p} d t
$$

and that $\left(\int_{0}^{T}\left(|Y(t)|^{p} \wedge N\right) d t\right)_{N \geq 1}$ is a Cauchy sequence in probability. As this sequence converges for all $\omega \in \Omega^{1}$ (possibly to infinity) we get that

(a) $\mathbb{P}^{1}\left(\left\{\omega \in \Omega^{1}: \int_{0}^{T}|Y(t, \omega)|^{p} d t<\infty\right\}\right)=1$,

(b) $\int_{0}^{T}|Y(t)|^{p} \chi_{\left\{\int_{0}^{T}|Y(s)|^{p} d s<\infty\right\}} d t \in \mathcal{C}_{0}\left(\int_{0}^{T}|X(t)|^{p} d t\right)$. 


\section{CHAPTER 3}

\section{Transference of SDEs}

In this chapter we apply the method from Chapter 2 to the Wiener space. The main technical result is Theorem 3.3 below and gives a functional map to move a BSDE from one stochastic basis to another one. For this we do not need any uniqueness of the solution of the BSDE that is moved. By using an independent copy of the Wiener space we generate in Chapter 6 below a twisted copy of our BSDE by this procedure. The comparison of the original BSDE with the twisted copy will yield to the notion of anisotropic smoothness. Theorem 3.3 might also be exploited to map a BSDE to the canonical path-space of continuous functions or from the canonical path-space back to some other space.

\subsection{Setting}

For $i=0,1$ assume complete probability spaces $\left(\Omega^{i}, \mathcal{F}^{i}, \mathbb{P}^{i}\right)$ hosting $d$-dimensional Brownian motions

$$
W^{i}=\left(W_{t}^{i}\right)_{t \in[0, T]}=\left(\left(W_{t, 1}^{i}, \ldots, W_{t, d}^{i}\right)^{\top}\right)_{t \in[0, T]},
$$

where all paths are assumed to be continuous and $W_{0}^{i} \equiv 0$. Taking the transposed vector means also that the Brownian motion is considered as column vector. Define the filtrations $\mathbb{F}^{i}=\left(\mathcal{F}_{t}^{i}\right)_{t \in[0, T]}$ by $\mathcal{F}_{t}^{i}:=\sigma\left(W_{s}^{i}: s \in[0, t]\right) \vee \mathcal{N}^{i}$ with $\mathcal{N}^{i}$ being the $\mathbb{P}^{i}$-null-sets. Replacing $\mathcal{F}^{i}$ by $\mathcal{F}_{T}^{i}$ we will assume that $\mathcal{F}^{i}=\mathcal{F}_{T}^{i}$. Furthermore, we equip $L_{2}\left([0, T] ; \mathbb{R}^{d}\right)$ with the orthonormal basis $\left(h_{k} \otimes e_{i}\right)_{k=0, i=1}^{\infty, d}$, where $\left(h_{k}\right)_{k=0}^{\infty}$ are the $L_{2}([0, T])$-normalized Haar functions 1 and $e_{1}, \ldots, e_{d}$ are the unit vectors of $\mathbb{R}^{d}$. The corresponding systems $\left(\xi_{k}^{i}\right)_{k \in I}$ of random variables from Section 2.1 are given by

$$
\mathcal{B}^{i}:=\left\{g_{k, j}^{i}: k \geq 0, j=1, \ldots, d\right\} \text { with } g_{k, j}^{i}:=\int_{0}^{T} h_{k}(t) d W_{t, j}^{i},
$$

where we take as the representative the finite differences of the $j$-th coordinate of $W^{i}$ generated by the Haar function $h_{k}$. Because all paths of $W^{i}$ are continuous we have

$$
\sigma\left(W_{t, j}^{i}: t \in[0, T] ; j=1, \ldots, d\right)=\sigma\left(g_{k, j}^{i}: k=0,1,2, \ldots \text { and } j=1, \ldots, d\right) .
$$

The predictable $\sigma$-algebras on $\left(\Omega^{i}, \mathcal{F}^{i}, \mathbb{P}^{i}, \mathbb{F}^{i}\right)$ are denoted by $\mathcal{P}^{i}$.

\footnotetext{
${ }^{1}$ The Haar functions are based on the dyadic intervals $\left(T \frac{l-1}{2^{L}}, T \frac{l}{2^{L}}\right]$ with $L=0,1,2, \ldots$ and $\left.l=1, \ldots, 2^{L}\right)$.
} 


\subsection{Results}

Before we state the main result we need two lemmas.

Lemma 3.1. One has $W_{t, j}^{1} \in \mathcal{C}_{0}\left(W_{t, j}^{0}\right)$ for $j=1, \ldots, d$ and $t \in[0, T]$ so that $\mathcal{C}_{0}\left(L_{0}\left(\Omega^{0}, \mathcal{F}_{t}^{0}, \mathbb{P}^{0}\right)\right) \subseteq L_{0}\left(\Omega^{1}, \mathcal{F}_{t}^{1}, \mathbb{P}^{1}\right)$ for $t \in[0, T]$.

Proof. The construction and Proposition 2.5(1) imply $W_{t, j}^{1} \in \mathcal{C}_{0}\left(W_{t, j}^{0}\right)$ whenever $t=T k / 2^{n}$ with $n=0,1,2, \ldots$ and $k=0, \ldots, 2^{n}$. For a $t \in(0, T)$ not of this form we find dyadic $t_{n} \in[0, T]$ with $t_{n} \rightarrow t$. Hence $W_{t_{n}, j}^{i} \rightarrow W_{t, j}^{i}$ for $i=0,1$ in probability and Proposition 2.5 (2) yields $W_{t, j}^{1} \in \mathcal{C}_{0}\left(W_{t, j}^{0}\right)$. The second part of the statement is a consequence of the first one.

Lemma 3.2. Assume that $K^{0} \in \mathcal{L}_{2}\left(\Omega_{T}^{0}, \mathcal{P}^{0}\right)$. Then, for all $j=1, \ldots, d$,

$$
\int_{0}^{T} K_{t}^{1} d W_{t, j}^{1} \in \mathcal{C}_{0}\left(\int_{0}^{T} K_{t}^{0} d W_{t, j}^{0}\right),
$$

where $K^{1} \in \mathcal{C}_{T}\left(K^{0}\right)$ is any $\mathcal{P}^{1}$-measurable representative.

Proof. Let $L \geq 1,0=t_{0}^{L}<\cdots<t_{L}^{L}=T$, and $\left(\varphi_{l}^{0, L}\right)_{l=1, \ldots, L}$ such that $\varphi_{l}^{0, L} \in \mathcal{L}_{2}\left(\Omega^{0}, \mathcal{F}_{t_{l-1}^{L}}^{0}\right)$, and $K_{t}^{0, L}:=\sum_{l=1}^{L} \varphi_{l}^{0, L_{1}^{L}} 1_{\left(t_{l-1}^{L}, t_{l}^{L}\right]}(t)$ such that

$$
\mathbb{E}^{0} \int_{0}^{T}\left|K_{t}^{0}-K_{t}^{0, L}\right|^{2} d t \rightarrow 0 \quad \text { as } \quad L \rightarrow \infty
$$

see [58, Lemma 3.2.4]. Using Proposition 2.5 and Lemma 3.1, letting $\varphi_{l}^{1, L} \in$ $\mathcal{C}_{0}\left(\varphi_{l}^{0, L}\right)$ and $K_{t}^{1, L}:=\sum_{l=1}^{L} \varphi_{l}^{1, L} 1_{\left(t_{l-1}^{L}, t_{l}^{L}\right]}(t)$, we get

$$
\begin{aligned}
\mathcal{C}_{0}\left(\int_{0}^{T} K_{t}^{0} d W_{t, j}^{0}\right) & =\lim _{L \rightarrow \infty} \mathcal{C}_{0}\left(\sum_{l=1}^{L} \varphi_{l}^{0, L}\left(W_{t_{l}^{L}, j}^{0}-W_{t_{l-1}^{L}, j}^{0}\right)\right) \\
& \ni \lim _{L \rightarrow \infty} \sum_{l=1}^{L} \varphi_{l}^{1, L}\left(W_{t_{l}^{L}, j}^{1}-W_{t_{l-1}^{L}, j}^{1}\right) \\
& =\int_{0}^{T} K_{t}^{1} d W_{t, j}^{1}
\end{aligned}
$$

where the limits are taken in $L_{2}\left(\Omega^{1}\right)$ and $K^{1}$ is a $\mathcal{P}^{1}$-measurable process that satisfies $\mathbb{E}^{1} \int_{0}^{T}\left|K_{t}^{1}-K_{t}^{1, L}\right|^{2} d t \rightarrow_{L} 0$. Because of Proposition 2.5 (7) we have $K^{1, L} \in$ $\mathcal{C}_{T}\left(K^{0, L}\right)$ so that $K^{1} \in \mathcal{C}_{T}\left(K^{0}\right)$ as well.

For integers $N, d \geq 1$ and $(\Omega, \mathbb{F}, \mathbb{P}, W)$ being one of the quadruples $\left(\Omega^{i}, \mathbb{F}^{i}, \mathbb{P}^{i}, W^{i}\right)$ we consider

$$
L_{t}=\xi+\int_{t}^{T} f\left(s, K_{s}\right) d s-\sum_{j=1}^{d} \int_{t}^{T} g_{j}\left(s, K_{s}\right) d W_{s, j}
$$

where

$(S 1) \xi \in \mathcal{L}_{0}(\Omega)$

$(S 2) f$ and $g_{j}$ are $\left(\mathcal{P}, \mathcal{B}\left(C\left(\mathbb{R}^{N}\right)\right)\right)$-measurable,

(S3) $L=\left(L_{t}\right)_{t \in[0, T]}, L_{t}: \Omega \rightarrow \mathbb{R}$, is continuous and $\mathbb{F}$-adapted, 
(S4) $K=\left(K_{t}\right)_{t \in[0 . T]}, K_{t}: \Omega \rightarrow \mathbb{R}^{N}$, is $\mathcal{P}$-measurable,

$(S 5) \mathbb{E} \int_{0}^{T}\left[\left|f\left(t, K_{t}\right)\right|+\left|g\left(t, K_{t}\right)\right|^{2}\right] d t<\infty$,

$(S 6)(\xi, f, g, K, L, W)$ satisfies 13 for $t \in[0, T] \mathbb{P}$-a.s.

Our main technical result is:

Theorem 3.3. Assume that $\left(\xi^{0}, f^{0}, g^{0}, K^{0}, L^{0}, W^{0}\right)$ satisfies $(S 1)-(S 6)$. Let $\xi^{1} \in$ $\mathcal{C}_{0}\left(\xi^{0}\right), f^{1} \in \mathcal{C}_{T}^{\mathbb{R}^{N}}\left(f^{0}\right)$ and $g_{j}^{1} \in \mathcal{C}_{T}^{\mathbb{R}^{N}}\left(g_{j}^{0}\right)$ be $\left(\mathcal{P}^{1}, \mathcal{B}\left(C\left(\mathbb{R}^{N}\right)\right)\right)$-measurable, $L^{1} \in$ $\mathcal{C}_{0}^{[0, T]}\left(L^{0}\right)$ be $\mathbb{F}^{1}$-adapted and $K_{l}^{1} \in \mathcal{C}_{T}\left(K_{l}^{0}\right)$ be $\mathcal{P}^{1}$-measurable for $l=1, \ldots, N$. Then $\left(\xi^{1}, f^{1}, g^{1}, K^{1}, L^{1}, W^{1}\right)$ satisfies conditions $(S 1)-(S 6)$.

Proof. The existence of suitable measurable representatives can be deduced from a combination of Lemma 3.1 and Propositions 2.12 and 2.13. Using Proposition 2.13(1) we have for $\phi \in\left\{f, g_{j}\right\}$ that $\left(\phi^{1}\left(t, K_{t}^{1}\right)\right)_{t \in[0, T]} \in \mathcal{C}_{T}\left(\left(\phi^{0}\left(t, K_{t}^{0}\right)\right)_{t \in[0, T]}\right)$. Continuing with Remark 2.14 (2) yields that condition (S5) is satisfied for $f^{1}\left(t, K_{t}^{1}\right)$ and $g^{1}\left(t, K_{t}^{1}\right)$. For a fixed $t \in[0, T]$ we have

$$
\mathcal{C}_{0}\left(L_{t}^{0}\right)=\mathcal{C}_{0}\left(\xi^{0}\right)+\mathcal{C}_{0}\left(\int_{t}^{T} f^{0}\left(s, K_{s}^{0}\right) d s\right)-\sum_{j=1}^{d} \mathcal{C}_{0}\left(\int_{t}^{T} g_{j}^{0}\left(s, K_{s}^{0}\right) d W_{s, j}^{0}\right) .
$$

Using Remark 2.14 (2) with $\phi(t, x)=x$, we have

$$
\int_{t}^{T} f^{1}\left(s, K_{s}^{1}\right) d s \in \mathcal{C}_{0}\left(\int_{t}^{T} f^{0}\left(s, K_{s}^{0}\right) d s\right)
$$

for all $t \in[0, T]$. Similarly Lemma 3.2 gives, for $t \in[0, T]$,

$$
\int_{t}^{T} g_{j}^{1}\left(s, K_{s}^{1}\right) d W_{s, j}^{1} \in \mathcal{C}_{0}\left(\int_{t}^{T} g_{j}^{0}\left(s, K_{s}^{0}\right) d W_{s, j}^{0}\right) .
$$

Later, in our application we need that certain properties of the generator transfer. For this purpose we use the following

REMARK 3.4. Assume that $h^{0}: \Omega_{T}^{0} \rightarrow C\left(\mathbb{R}^{N}\right)$ is $\left(\mathcal{P}^{0}, \mathcal{B}\left(C\left(\mathbb{R}^{N}\right)\right)\right)$-measurable and $h^{1} \in \mathcal{C}_{T}^{\mathbb{R}^{N}}\left(h^{0}\right)$ is $\left(\mathcal{P}^{1}, \mathcal{B}\left(C\left(\mathbb{R}^{N}\right)\right)\right)$-measurable. Then the following holds:

(1) $h^{0}(\cdot, \cdot, 0) \stackrel{d}{=} h^{1}(\cdot, \cdot, 0)$ with respect to $\lambda \times \mathbb{P}^{0}$ and $\lambda \times \mathbb{P}^{1}$.

(2) Given a continuous $H: \mathbb{R}^{N} \times \mathbb{R}^{N} \rightarrow[0, \infty)$ such that, for all $\left(t, \omega^{0}, x_{0}, x_{1}\right)$,

$$
\left|h^{0}\left(t, \omega^{0}, x_{0}\right)-h^{0}\left(t, \omega^{0}, x_{1}\right)\right| \leq H\left(x_{0}, x_{1}\right),
$$

then we can choose $h^{1}$ such that, for all $\left(t, \omega^{1}, x_{0}, x_{1}\right)$,

$$
\left|h^{1}\left(t, \omega^{1}, x_{0}\right)-h^{1}\left(t, \omega^{1}, x_{1}\right)\right| \leq H\left(x_{0}, x_{1}\right) .
$$

Proof. (1) follows from Definition 2.9 and Proposition 2.5.

(2) Given $x \in \mathbb{R}^{N}$, we have by construction $h^{1}(x) \in \mathcal{C}_{T}\left(h^{0}(x)\right)$, so that

$$
\left(h^{1}\left(x_{0}\right), h^{1}\left(x_{1}\right)\right) \stackrel{d}{=}\left(h^{0}\left(x_{0}\right), h^{0}\left(x_{1}\right)\right) \quad \text { for all } \quad x_{0}, x_{1} \in \mathbb{R}^{N} .
$$

This implies that

$$
\left\|h^{1}\left(x_{0}\right)-h^{1}\left(x_{1}\right)\right\|_{L_{\infty}\left(\Omega_{T}^{1}\right)}=\left\|h^{0}\left(x_{0}\right)-h^{0}\left(x_{1}\right)\right\|_{L_{\infty}\left(\Omega_{T}^{0}\right)} \leq H\left(x_{0}, x_{1}\right) .
$$

Hence, letting 


$$
\begin{array}{r}
\Omega_{T, 0}^{1} \\
:=\quad \begin{array}{r}
\left\{t, \omega^{1}\right) \in[0, T] \times \Omega^{1}:\left|h^{1}\left(t, \omega^{1}, x_{0}\right)-h^{1}\left(t, \omega^{1}, x_{1}\right)\right| \leq H\left(x_{0}, x_{1}\right) \\
\left.\quad \text { for all } x_{0}, x_{1} \in \mathbb{R}^{N}\right\}
\end{array} \\
=\quad\left\{\left(t, \omega^{1}\right) \in[0, T] \times \Omega^{1}:\left|h^{1}\left(t, \omega^{1}, x_{0}\right)-h^{1}\left(t, \omega^{1}, x_{1}\right)\right| \leq H\left(x_{0}, x_{1}\right)\right. \\
\left.\quad \text { for all } x_{0}, x_{1} \in \mathbb{Q}^{N}\right\},
\end{array}
$$

we have that $\Omega_{T, 0}^{1} \in \mathcal{P}^{1}$ and $\mathbb{P}_{T}^{1}\left(\Omega_{T, 0}^{1}\right)=1$. Setting

$$
\tilde{h}^{1}:=\chi_{\Omega_{T, 0}^{1}} h^{1} \in \mathcal{C}_{T}^{\mathbb{R}^{N}}\left(h^{0}\right),
$$

we obtain a $\left(\mathcal{P}^{1}, \mathcal{B}\left(C\left(\mathbb{R}^{N}\right)\right)\right)$-measurable map as desired. 


\section{CHAPTER 4}

\section{Anisotropic Besov Spaces on the Wiener Space}

In this chapter we introduce anisotropic Besov spaces on the Wiener space by the decoupling method from Chapter 2. The spaces are designed such that nonlinear conditional expectations, that are generated by BSDEs, map these spaces into itself (see Chapter 6). This fact will provide variational estimates for solutions to BSDEs. Our approach to define anisotropic Besov spaces is very flexible as it allows different types of spaces, including the classical spaces obtained by the real interpolation method.

\subsection{Classical Besov spaces on the Wiener space}

In this section we introduce the classical Besov spaces on the Wiener space obtained by the real interpolation method. To do so we first recall the real interpolation method and the concept of Banach space valued random variables.

4.1.1. Real interpolation method. For detailed information about the real interpolation method the reader is referred (for example) to the monographs $\mathbf{1 0}$, [11, or 86. To define the method in the general context, we say that two Banach spaces $\left(E_{0}, E_{1}\right)$ form a compatible couple provided that there is a Banach space $X$ such that $E_{0}$ and $E_{1}$ are continuously embedded into $X$. By this assumption we can define $E_{0}+E_{1}:=\left\{x=x_{0}+x_{1}, x_{0} \in E_{0}, x_{1} \in E_{1}\right\}$, where the sum is taken in $X$. Afterwards, $X$ can be taken to be $E_{0}+E_{1}$ if

$$
\|x\|_{E_{0}+E_{1}}:=\inf \left\{\left\|x_{0}\right\|_{E_{0}}+\left\|x_{1}\right\|_{E_{1}}: x=x_{0}+x_{1}, x_{i} \in E_{i}\right\},
$$

see [11, Lemma 2.3.1]. Assuming additionally that $E_{1}$ is continuously embedded into $E_{0}$, which is our typical case later, we can take $X=E_{0}$ itself.

Definition 4.1. Given a compatible couple $\left(E_{0}, E_{1}\right)$ of Banach spaces and $x \in$ $E_{0}+E_{1}$ and $t>0$, we define the $K$-functional

$$
K\left(x, t ; E_{0}, E_{1}\right):=\inf \left\{\left\|x_{0}\right\|_{E_{0}}+t\left\|x_{1}\right\|_{E_{1}}: x=x_{0}+x_{1}, x_{i} \in E_{i}\right\} .
$$

For $\theta \in(0,1)$ and $q \in[1, \infty]$ we let $\left(E_{0}, E_{1}\right)_{\theta, q}$ be the real interpolation space of all $x \in E_{0}+E_{1}$ such that

$$
\|x\|_{\left(E_{0}, E_{1}\right)_{\theta, q}}:=\left\|t^{-\theta} K\left(x, t ; E_{0}, E_{1}\right)\right\|_{L_{q}\left((0, \infty), \frac{d t}{t}\right)}<\infty .
$$

To explain the role of the parameters $(\theta, q)$ let us begin with some properties of the real interpolation method: 
Proposition 4.2 ([86, Section 1.3.3]). Let $\left(E_{0}, E_{1}\right)$ be a compatible couple of Banach spaces, $\theta \in(0,1)$, and $q \in[1, \infty]$. Then one has the following:

(1) $\left(E_{0}, E_{1}\right)_{\theta, q}=\left(E_{1}, E_{0}\right)_{1-\theta, q}$ for $\theta \in(0,1)$ and $q \in[1, \infty]$.

(2) $\left(E_{0}, E_{1}\right)_{\theta, q_{0}} \subseteq\left(E_{0}, E_{1}\right)_{\theta, q_{1}}$ for $\theta \in(0,1)$ and $1 \leq q_{0} \leq q_{1} \leq \infty$.

(3) If $E_{1}$ is continuously embedded into $E_{0}$, then

$\left(E_{0}, E_{1}\right)_{\theta_{0}, q_{0}} \subseteq\left(E_{0}, E_{1}\right)_{\theta_{1}, q_{1}}$ for $0<\theta_{1}<\theta_{0}<1$ and $q_{0}, q_{1} \in[1, \infty]$.

If we assume that $E_{0}=L_{p}$ and that $E_{1} \subseteq L_{p}$ is a subspace that describes certain regularity properties with $\|\cdot\|_{p} \leq\|\cdot\|_{E_{1}}$, then we are in the position of Proposition $4.2(3)$. The parameter $\theta$ becomes the main regularity parameter, and for a fixed $\theta$, the parameter $q$ becomes another regularity parameter, that can be interpreted as a fine-tuning parameter. The ordering in Proposition 4.2 (3) is also called lexicographical ordering.

4.1.2. Banach space valued random variables. Given a separable Banach space $X$ and a probability space $(\Omega, \mathcal{F}, \mathbb{P})$, a map $F: \Omega \rightarrow X$ is measurable if it is measurable with respect to $(\mathcal{F}, \mathcal{B}(X))$, where $\mathcal{B}(X)$ is the Borel $\sigma$-algebra generated by the norm open sets in $X$. For $p \in(0, \infty]$ we define

$$
\begin{aligned}
\|F\|_{L_{p}^{X}} & =\|F\|_{L_{p}^{X}(\Omega)}:=\|\| F\left\|_{X}\right\|_{p}, \\
\mathcal{L}_{p}^{X}(\Omega) & :=\left\{F: \Omega \rightarrow X \text { measurable, }\|F\|_{L_{p}^{X}}<\infty\right\},
\end{aligned}
$$

and let $L_{p}^{X}(\Omega)$ be the corresponding space of equivalence classes where we identify random variables $F, G: \Omega \rightarrow X$ whenever $\mathbb{P}(F=G)=1$.

4.1.3. Besov spaces on the abstract Wiener space. We assume a separable Hilbert space $H$, a complete probability space $(\Omega, \mathcal{F}, \mathbb{P})$, and an iso-normal family of Gaussian random variables $\left(g_{h}\right)_{h \in H}, g_{h}: \Omega \rightarrow \mathbb{R}$, i.e.

$$
\mathbb{E} g_{h}=0 \text { and } \mathbb{E} g_{h} g_{k}=\langle h, k\rangle \text { for all } h, k \in H .
$$

For $\alpha_{1}, \ldots, \alpha_{n} \in \mathbb{R}$ and $h_{1}, \ldots, h_{n} \in H$ this implies

$$
\alpha_{1} g_{h_{1}}+\cdots+\alpha_{n} g_{h_{n}}=g_{\alpha_{1} h_{1}+\cdots \alpha_{n} h_{n}} \text { a.s., }
$$

that means that $\left(g_{h}\right)_{h \in H}$ is a Gaussian process. W.l.o.g. we may assume that $\mathcal{F}$ is the completion of $\sigma\left(g_{h}: h \in H\right)$. Let $\left(\mathbf{h}_{n}\right)_{n=0}^{\infty}$ be the normalised Hermitepolynomials, i.e. $\mathbf{h}_{n}: \mathbb{R} \rightarrow \mathbb{R}$ with $\mathbf{h}_{0} \equiv 1$ and

$$
\mathbf{h}_{n}(x):=(-1)^{n} \frac{1}{\sqrt{n !}} e^{\frac{x^{2}}{2}} \frac{d^{n}}{d x^{n}} e^{-\frac{x^{2}}{2}} \quad \text { for } n \geq 1 .
$$

Letting $\gamma_{N}$ be the standard Gaussian measure on $\mathbb{R}^{N}$, the Hermite polynomials form an orthogonal basis in $L_{2}\left(\mathbb{R}, \gamma_{1}\right)$. Now we are in a position to define the Wiener chaos:

Definition 4.3. Let $\left(e_{k}\right)_{k \in I} \subseteq H$ be an orthogonal basis of $H$. Given $n \geq 1$, the space (of equivalence classes)

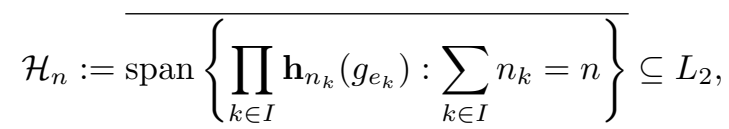

where the closure is taken in $L_{2}$, is the $n$-th Wiener chaos. For $n=0$ we let $\mathcal{H}_{0}$ be space of all equivalence classes that contain a constant. 
The space $\mathcal{H}_{n}$ does not depend on the choice of the orthogonal basis $\left(e_{k}\right)_{k \in I} \subseteq H$. Moreover, one has the fundamental Wiener chaos expansion

$$
L_{2}(\Omega, \mathcal{F}, \mathbb{P})=\oplus_{n=0}^{\infty} \mathcal{H}_{n},
$$

in particular the spaces $\mathcal{H}_{n}$ and $\mathcal{H}_{m}$ are orthogonal for $n \neq m$. Letting

$$
P_{n}: L_{2} \rightarrow \mathcal{H}_{n} \subseteq L_{2}
$$

be the orthogonal projection onto the $n$-th chaos, we define the Hilbert space

$$
\mathbb{D}_{1,2}:=\left\{\xi \in L_{2}:\|\xi\|_{\mathbb{D}_{1,2}}^{2}:=\sum_{n=0}^{\infty}(n+1)\left\|P_{n} \xi\right\|_{2}^{2}<\infty\right\} .
$$

As Malliavin derivative we take $D: \mathbb{D}_{1,2} \rightarrow L_{2}^{H}$ with

$$
D\left(\prod_{k \in I} \mathbf{h}_{n_{k}}\left(g_{e_{k}}\right)\right):=\sum_{l \in I} \prod_{k \neq l} \mathbf{h}_{n_{k}}\left(g_{e_{k}}\right) h_{n_{l}}^{\prime}\left(g_{e_{l}}\right) e_{l} .
$$

By definition the elements of $\mathbb{D}_{1,2}$ are equivalence classes from $L_{2}, D$ is defined on equivalence classes and maps to equivalence classes in $L_{2}^{H}$. When needed, we interpret $D F$ as an element of $\mathcal{L}_{2}^{H}(\Omega)$ or of $\mathcal{L}_{2}^{\mathbb{R}^{d}}(\Omega \times[0, T])$ if $H=L_{2}^{\mathbb{R}^{d}}([0, T])$. It is known that

$$
D f\left(g_{h_{1}}, \ldots, g_{h_{n}}\right)=\sum_{k=1}^{n} \frac{\partial f}{\partial x_{k}}\left(g_{h_{1}}, \ldots, g_{h_{n}}\right) h_{k}
$$

for (say) $f \in C_{b}^{\infty}\left(\mathbb{R}^{n}\right)$ and $h_{1}, \ldots, h_{n} \in H$. If $p \in(2, \infty)$, then we let

$$
\mathbb{D}_{1, p}:=\left\{\xi \in \mathbb{D}_{1,2}:\|\xi\|_{\mathbb{D}_{1, p}}^{p}:=\|\xi\|_{p}^{p}+\|D \xi\|_{L_{p}^{H}}^{p}<\infty\right\},
$$

which is consistent with the case $p=2$. The spaces $\mathbb{D}_{1, p}$ are known to be Banach spaces (as $p \in[2, \infty)$ one can use the completeness of $\mathbb{D}_{1,2}$ and $L_{p}$, and Fatou's lemma). Moreover, we set

$$
\mathbb{B}_{p, q}^{\theta}:=\left(L_{p}, \mathbb{D}_{1, p}\right)_{\theta, q} .
$$

In the case $\operatorname{dim}(H)=n$ we identify $L_{2}$ with $L_{2}\left(\mathbb{R}^{n}, \mathcal{B}\left(\mathbb{R}^{n}\right), \gamma_{n}\right)$ and use the family $g_{\left(\xi_{1}, \ldots, \xi_{n}\right)}: \mathbb{R}^{n} \rightarrow \mathbb{R}$ given by

$$
g_{\left(\xi_{1}, \ldots, \xi_{n}\right)}\left(x_{1}, \ldots, x_{n}\right):=\xi_{1} x_{1}+\cdots+\xi_{n} x_{n} .
$$

We denote these particular Besov spaces by $\mathbb{B}_{p, q}^{\theta}\left(\mathbb{R}^{n}, \gamma_{n}\right)$. To motivate the decoupling method and the corresponding Besov spaces introduced in Sections 4.2 and 4.3 below, we describe the spaces $\mathbb{B}_{p, q}^{\theta}\left(\mathbb{R}^{n}, \gamma_{n}\right)$ by decoupling:

Theorem 4.4 ([46. Theorem 3.1]). Let $p \in[2, \infty), \theta \in(0,1), q \in[1, \infty]$, and $f \in L_{p}\left(\mathbb{R}^{n}, \gamma_{n}\right)$. Then

$$
\|f\|_{\mathbb{B}_{p, q}^{\theta}\left(\mathbb{R}^{n}, \gamma_{n}\right)} \sim_{c[4.4}\|f\|_{p}+\left\|(1-t)^{-\frac{\theta}{2}}\right\| f(g)-f\left(t g+\sqrt{1-t^{2}} g^{\prime}\right)\left\|_{p}\right\|_{L_{q}\left([0,1), \frac{d t}{1-t}\right)}
$$

where $c \sqrt{4.4} \geq 1$ depends uniquely on $(p, \theta, q)$, and $g$ and $g^{\prime}$ are independent $\mathbb{R}^{n}$ valued random variables with law $\gamma_{n}$. 
Proof. To derive our formulation from that one in 46] we consider an $n$ dimensional Brownian motion $\left(W_{t}\right)_{t \in[0,1]}$ with respect to a filtration $\left(\mathcal{F}_{t}\right)_{t \in[0,1]}$ and notice (cf. Lemma 4.23 below) that

$$
\left\|f\left(W_{1}\right)-\mathbb{E}\left(f\left(W_{1}\right) \mid \mathcal{F}_{t}\right)\right\|_{p} \sim_{2}\left\|f\left(W_{1}\right)-f\left(W_{t}+\left[W_{1}^{\prime}-W_{t}^{\prime}\right]\right)\right\|_{p},
$$

where $\left(W_{t}^{\prime}\right)_{t \in[0,1]}$ is an independent copy of $\left(W_{t}\right)_{t \in[0,1]}$. If we set,

$$
W_{1}^{(t, 1]}:=W_{t}+\left[W_{1}^{\prime}-W_{t}^{\prime}\right]
$$

then we obtain from [46. Theorem 3.1] and [16 the equivalence

$$
\|f\|_{\mathbb{B}_{p, q}^{\theta}\left(\mathbb{R}^{n}, \gamma_{n}\right)} \sim\|f\|_{p}+\left\|(1-t)^{-\frac{\theta}{2}}\right\| f\left(W_{1}\right)-f\left(W_{1}^{(t, 1]}\right)\left\|_{p}\right\|_{L_{q}\left([0,1), \frac{d t}{1-t}\right)} .
$$

Finally, because $\left(W_{1}, W_{1}^{(t, 1]}\right)$ and $\left(g, t g+\sqrt{1-t^{2}} g^{\prime}\right)$ have the same law, we conclude the proof.

Theorem 4.4 generalizes results from [45. In the formulation of Theorem 4.4 we use an isotropic decoupling, which means that the Gaussian structure $g$ is uniformly replaced by $t g+\sqrt{1-t^{2}} g^{\prime}$. Instead, the right-hand side of (17) uses an anisotropic decoupling in the larger Wiener space based on the Brownian motion $\left(W_{s}\right)_{s \in[0,1]}$ as the replacement of $\left(W_{s}\right)_{s \in[0,1]}$ is $\left(W_{s}^{(t, 1]}\right)_{s \in[0,1]}$, i.e. only part of the Gaussian structure is decoupled. This anisotropic decoupling in 17$]$ is the key idea of [40] to obtain estimates for the variation of BSDEs, an isotropic decoupling in the larger Wiener space could not be used in this context as explained in Remark 4.21 below.

\subsection{Setting}

For $d \geq 1$ and $T>0$ we fix two standard $d$-dimensional Brownian motions $W=$ $\left(W_{t}\right)_{t \in[0, T]}$ and $W^{\prime}=\left(W_{t}^{\prime}\right)_{t \in[0, T]}$, where all paths are assumed to be continuous with $W_{0} \equiv 0$ and $W_{0}^{\prime} \equiv 0$, that are defined on complete probability spaces $(\Omega, \mathcal{F}, \mathbb{P})$ and $\left(\Omega^{\prime}, \mathcal{F}^{\prime}, \mathbb{P}^{\prime}\right)$, where $\mathcal{F}$ and $\mathcal{F}^{\prime}$ are the completions of $\sigma\left(W_{t}: t \in[0, T]\right)$ and $\sigma\left(W_{t}^{\prime}: t \in[0, T]\right)$, respectively. We let

$$
\bar{\Omega}:=\Omega \times \Omega^{\prime}, \quad \overline{\mathbb{P}}:=\mathbb{P} \times \mathbb{P}^{\prime}, \quad \overline{\mathcal{F}}:=\overline{\mathcal{F} \otimes \mathcal{F}^{\prime}} \overline{\mathbb{P}}
$$

and extend the Brownian motions $W$ and $W^{\prime}$ canonically to $\Omega \times \Omega^{\prime}$. Given a measurable function $\varphi:(0, T] \rightarrow[0,1]$, we let

$$
W_{t}^{\varphi}:=\int_{0}^{t}\left[1-\varphi(u)^{2}\right]^{\frac{1}{2}} d W_{u}+\int_{0}^{t} \varphi(u) d W_{u}^{\prime}
$$

and again assume continuity for all trajectories and that $W_{0}^{\varphi} \equiv 0$. For example, for $0 \leq a<b \leq T$, this definition yields to

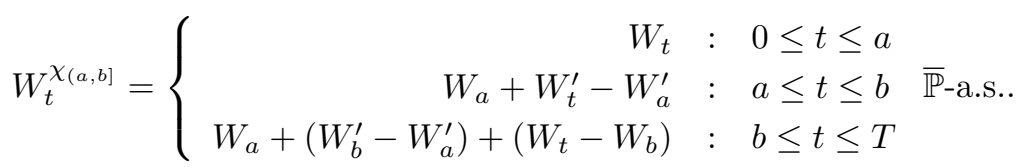

The process $W^{\varphi}$ is a standard Brownian motion and $\left(\mathcal{F}_{t}^{\varphi}\right)_{t \in[0, T]}$ will denote its $\overline{\mathbb{P}}$-augmented natural filtration, i.e.

$$
\mathcal{F}_{t}^{\varphi}:=\sigma\left(W_{s}^{\varphi}: s \in[0, t]\right) \vee \overline{\mathcal{N}},
$$


where $\overline{\mathcal{N}}$ are the $\overline{\mathbb{P}}$-null-sets from $\overline{\mathcal{F}}$. Identifying $a \in[0,1]$ with the function $\varphi$ : $(0, T] \rightarrow[0,1]$ that is constant $a$, we agree to take the versions

$$
W^{0}=W \quad \text { and } \quad W^{1}=W^{\prime} .
$$

To apply the results from Chapter 3 we use the pairing between

$$
\left(\bar{\Omega}, \mathcal{F}^{0}, \overline{\mathbb{P}}, \mathbb{F}^{0}, W^{0}, \mathcal{B}^{0}\right) \text { and }\left(\bar{\Omega}, \mathcal{F}^{\varphi}, \overline{\mathbb{P}}, \mathbb{F}^{\varphi}, W^{\varphi}, \mathcal{B}^{\varphi}\right),
$$

where $\mathcal{F}^{\psi}:=\mathcal{F}_{T}^{\psi}, \mathbb{F}^{\psi}=\left(\mathcal{F}_{t}^{\psi}\right)_{t \in[0, T]}$, and $\mathcal{B}^{\psi}$ is defined like in 12 for $\psi \in\{0, \varphi\}$. The corresponding operators $\mathcal{C}_{S}$ and $\mathcal{C}_{S}^{M}$ from Definitions 2.3 and 2.9 are denoted by $\mathcal{C}_{S}(\varphi)$ and $\mathcal{C}_{S}^{M}(\varphi)$, respectively.

Convention 4.5.

(1) If needed, we extend a random variable $\xi: \Omega \rightarrow \mathbb{R}$ to $\widetilde{\xi}: \bar{\Omega} \rightarrow \mathbb{R}$ by $\widetilde{\xi}\left(\omega, \omega^{\prime}\right):=$ $\xi(\omega)$. The extension $\widetilde{\xi}$ is measurable with respect to $\mathcal{F}^{0}$. In this sense we can apply the operator

$$
\mathcal{C}_{0}(\varphi): L_{0}\left(\bar{\Omega}, \mathcal{F}^{0}\right) \rightarrow L_{0}\left(\bar{\Omega}, \mathcal{F}^{\varphi}\right)
$$

to $\xi$. To simplify the notation, $\widetilde{\xi}$ will be usually denoted by $\xi$ as well.

(2) For a random variable $\xi: \Omega \rightarrow \mathbb{R}$ we denote by $\xi^{\varphi}$ the elements of $\mathcal{C}_{0}(\varphi)(\xi)$ and, for $0 \leq a<b \leq T$, by $\xi^{(a, b]}$ the random variable $\xi^{\chi_{(a, b]}}$, i.e.

$$
\xi^{\varphi} \in \mathcal{C}_{0}(\varphi)(\xi) \text { and } \xi^{(a, b]}:=\xi^{\chi_{(a, b]}} .
$$

Because of Lemma 3.1 this notation is consistent with the definition from (18).

\subsection{Definition of anisotropic Besov spaces}

We start by defining the parameter space

$$
\mathfrak{D}:=\left\{\psi \in \mathcal{L}_{2}((0, T]): 0 \leq \psi \leq 1\right\}
$$

equipped with the pseudo-metric 1

$$
\delta(\varphi, \psi):=\|\varphi-\psi\|_{L_{2}((0, T])} .
$$

To define our Besov spaces we need some preparations.

LEMMA 4.6.

(1) For $\varphi, \psi \in \mathfrak{D}, k \geq 0$, and $i \in\{1, \ldots, d\}$ one has that

$$
\mathbb{E}\left|g_{k, i}^{\varphi}-g_{k, i}^{\psi}\right|^{2} \leq 2\left\|h_{k}\right\|_{\infty}^{2} \int_{0}^{T}\left|\varphi(t)^{2}-\psi(t)^{2}\right| d t .
$$

(2) If $\varphi_{n}, \varphi \in \mathfrak{D}$ are such that $\lim _{n} \delta\left(\varphi_{n}, \varphi\right)=0$, then

$$
\lim _{n} \mathbb{E}\left|g_{k, i}^{\varphi_{n}}-g_{k, i}^{\varphi}\right|^{2}=0 \text {. }
$$

Proof. (1) Starting from the corresponding definitions we get

$$
\begin{aligned}
& \mathbb{E}\left|g_{k, i}^{\varphi}-g_{k, i}^{\psi}\right|^{2} \\
= & \mathbb{E}\left|\int_{0}^{T} h_{k}(t)\left\langle e_{i}, d W_{t}^{\varphi}\right\rangle-\int_{0}^{T} h_{k}(t)\left\langle e_{i}, d W_{t}^{\psi}\right\rangle\right|^{2}
\end{aligned}
$$

\footnotetext{
${ }^{1}$ Here we only have a pseudo-metric as we do not work with the equivalence classes.
} 


$$
\begin{aligned}
= & \mathbb{E} \mid \int_{0}^{T} h_{k}(t) \sqrt{1-\varphi(t)^{2}}\left\langle e_{i}, d W_{t}\right\rangle+\int_{0}^{T} h_{k}(t) \varphi(t)\left\langle e_{i}, d W_{t}^{\prime}\right\rangle \\
& -\int_{0}^{T} h_{k}(t) \sqrt{1-\psi(t)^{2}}\left\langle e_{i}, d W_{t}\right\rangle-\left.\int_{0}^{T} h_{k}(t) \psi(t)\left\langle e_{i}, d W_{t}^{\prime}\right\rangle\right|^{2} \\
= & \int_{0}^{T} h_{k}(t)^{2}\left[\sqrt{1-\varphi(t)^{2}}-\sqrt{1-\psi(t)^{2}}\right]^{2} d t \\
& +\int_{0}^{T} h_{k}(t)^{2}[\varphi(t)-\psi(t)]^{2} d t \\
\leq \quad & \int_{0}^{T} h_{k}(t)^{2}\left|\varphi(t)^{2}-\psi(t)^{2}\right| d t+\int_{0}^{T} h_{k}(t)^{2}[\varphi(t)-\psi(t)]^{2} d t
\end{aligned}
$$

(2) If we assume that $\lim _{n} \delta\left(\varphi_{n}, \varphi\right)=0$, then $\varphi_{n} \rightarrow \varphi$ in probability with respect to the normalized Lebesgue measure on $[0, T]$ and therefore $\left|\varphi_{n}^{2}-\varphi^{2}\right| \rightarrow 0$ in probability as well. The boundedness $\left|\varphi_{n}(t)\right| \leq 1$ and $|\varphi(t)| \leq 1$ yields to $\lim _{n} \int_{0}^{T}\left|\varphi(t)^{2}-\varphi_{n}(t)^{2}\right| d t=0$ and we can apply part $(1)$

LEMmA 4.7. Let $p \in(0, \infty)$ and $\xi \in \mathcal{L}_{p}(\Omega, \mathcal{F}, \mathbb{P})$. Then $\delta\left(\varphi_{n}, \varphi\right) \rightarrow_{n} 0$ implies that

$$
\lim _{n}\left\|\xi^{\varphi_{n}}-\xi^{\varphi}\right\|_{p}=0 \text {. }
$$

Proof. (a) Assume that $\xi$ is bounded. Given $\varepsilon>0$ we find $N \geq 1, f \in$ $C_{b}\left(\mathbb{R}^{N}\right)$, and $\left(\gamma_{i}\right)_{i=1}^{N} \subset \mathcal{B}^{0}$ such that $\left\|\xi-f\left(\gamma_{1}, \ldots, \gamma_{N}\right)\right\|_{p}<\varepsilon$. Then, by Proposition 2.5 .

$$
\begin{aligned}
& \frac{1}{c_{p}}\left\|\xi^{\varphi_{n}}-\xi^{\varphi}\right\|_{p} \\
\leq \quad & \left\|\xi^{\varphi_{n}}-f\left(\gamma_{1}^{\varphi_{n}}, \ldots, \gamma_{N}^{\varphi_{n}}\right)\right\|_{p}+\left\|f\left(\gamma_{1}^{\varphi_{n}}, \ldots, \gamma_{N}^{\varphi_{n}}\right)-f\left(\gamma_{1}^{\varphi}, \ldots, \gamma_{N}^{\varphi}\right)\right\|_{p} \\
& +\left\|f\left(\gamma_{1}^{\varphi}, \ldots, \gamma_{N}^{\varphi}\right)-\xi^{\varphi}\right\|_{p} \\
\leq \quad & 2 \varepsilon+\left\|f\left(\gamma_{1}^{\varphi_{n}}, \ldots, \gamma_{N}^{\varphi_{n}}\right)-f\left(\gamma_{1}^{\varphi}, \ldots, \gamma_{N}^{\varphi}\right)\right\|_{p} .
\end{aligned}
$$

We can conclude by $\lim _{n}\left\|f\left(\gamma_{1}^{\varphi_{n}}, \ldots, \gamma_{N}^{\varphi_{n}}\right)-f\left(\gamma_{1}^{\varphi}, \ldots, \gamma_{N}^{\varphi}\right)\right\|_{p}=0$ which follows by Lemma 4.6.

(b) Assuming a general $\xi \in \mathcal{L}_{p}$, we let $\xi^{L}:=(-L) \vee \xi \wedge L$ for $L>0$ and obtain, again by Proposition 2.5 .

$$
\begin{aligned}
& \frac{1}{c_{p}}\left\|\xi^{\varphi_{n}}-\xi^{\varphi}\right\|_{p} \\
\leq & \left\|\xi^{\varphi_{n}}-\left(\xi^{L}\right)^{\varphi_{n}}\right\|_{p}+\left\|\left(\xi^{L}\right)^{\varphi_{n}}-\left(\xi^{L}\right)^{\varphi}\right\|_{p}+\left\|\left(\xi^{L}\right)^{\varphi}-\xi^{\varphi}\right\|_{p} \\
= & 2\left\|\xi-\xi^{L}\right\|_{p}+\left\|\left(\xi^{L}\right)^{\varphi_{n}}-\left(\xi^{L}\right)^{\varphi}\right\|_{p} .
\end{aligned}
$$

Given $\varepsilon>0$ we find an $L>0$ such that $2\left\|\xi-\xi^{L}\right\|_{p} \leq \varepsilon$, so that

$$
\frac{1}{c_{p}} \limsup _{n}\left\|\xi^{\varphi_{n}}-\xi^{\varphi}\right\|_{p} \leq \varepsilon+\lim _{n}\left\|\left(\xi^{L}\right)^{\varphi_{n}}-\left(\xi^{L}\right)^{\varphi}\right\|_{p} \leq \varepsilon .
$$

Because $\varepsilon>0$ was arbitrary, $\lim _{n}\left\|\xi^{\varphi_{n}}-\xi^{\varphi}\right\|_{p}=0$.

As a trivial by-product we get that $\xi^{\varphi}=\xi^{\psi} \overline{\mathbb{P}}$-a.s. if $\varphi=\psi$ a.e. Now it is convenient to turn $\mathfrak{D}$ into a complete separable metric space. 
Definition 4.8. We define the metric space $(\Delta, \delta)$ as the equivalence classes of the pseudo-metric space $(\mathfrak{D}, \delta)$ with

$$
\mathfrak{D}=\left\{\psi \in \mathcal{L}_{2}((0, T]): 0 \leq \psi \leq 1\right\} \quad \text { and } \quad \delta(\varphi, \psi)=\|\varphi-\psi\|_{L_{2}((0, T])} .
$$

Fixing $p \in(0, \infty)$ and $\xi \in \mathcal{L}_{p}(\Omega, \mathcal{F}, \mathbb{P})$, we obtain a well-defined map

$$
F_{\xi, p}: \Delta \rightarrow[0, \infty) \text { by } \varphi \rightarrow\left\|\xi-\xi^{\varphi}\right\|_{p} .
$$

Directly from Lemma 4.7 we get

Lemma 4.9. For $p \in(0, \infty)$ and $\xi \in \mathcal{L}_{p}(\Omega, \mathcal{F}, \mathbb{P})$ the map $F_{\xi, p}: \Delta \rightarrow[0, \infty)$ is continuous.

Proof. For $p \in[1, \infty)$ and $\varphi_{n} \rightarrow \varphi$ we get that

$$
\left|\left\|\xi-\xi^{\varphi_{n}}\right\|_{p}-\left\|\xi-\xi^{\varphi}\right\|_{p}\right| \leq\left\|\xi^{\varphi_{n}}-\xi^{\varphi}\right\|_{p} \rightarrow 0
$$

as $n \rightarrow \infty$. In the case $p \in(0,1)$ we use

$$
\left|\mathbb{E}\left[\left|\xi^{\varphi_{n}}-\xi\right|^{p}-\left|\xi^{\varphi}-\xi\right|^{p}\right]\right| \leq \mathbb{E}\left|\xi^{\varphi_{n}}-\xi^{\varphi}\right|^{p} .
$$

DeFinition 4.10. Let $C^{+}(\Delta)$ be the space of all non-negative continuous functions $F: \Delta \rightarrow[0, \infty)$. A functional $\Phi: C^{+}(\Delta) \rightarrow[0, \infty]$ is called admissible provided that

(A1) $\Phi(F+G) \leq \Phi(F)+\Phi(G)$,

(A2) $\Phi(\lambda F)=\lambda \Phi(F)$ for $\lambda \geq 0$,

(A3) $\Phi(F) \leq \Phi(G)$ for $0 \leq F \leq G$,

(A4) $\Phi(F) \leq \lim \sup _{n} \Phi\left(F_{n}\right)$ for $\sup _{\varphi \in \Delta}\left|F_{n}(\varphi)-F(\varphi)\right| \rightarrow_{n} 0$.

ExAmple 4.11. Let $A \subseteq \Delta$ be non-empty and let $\alpha: A \rightarrow(0, \infty)$ be an arbitrary weight. Then the functional

$$
\Phi(F):=\sup _{\varphi \in A} \frac{F(\varphi)}{\alpha(\varphi)}
$$

is admissible. As (A1)-(A3) are obvious, we only check (A4). From $F(\varphi) \leq$ $\lim \sup _{n} F_{n}(\varphi)$ we complete the proof by

$$
\frac{F(\varphi)}{\alpha(\varphi)} \leq \limsup _{n}\left[\sup _{\psi \in A} \frac{F_{n}(\psi)}{\alpha(\psi)}\right] .
$$

Definition 4.12. For $p \in(0, \infty), \xi \in L_{p}(\Omega)$, and an admissible $\Phi: C^{+}(\Delta) \rightarrow$ $[0, \infty]$ we let $\xi \in \mathbb{B}_{p}^{\Phi}$ provided that $\Phi\left(\varphi \rightarrow\left\|\xi-\xi^{\varphi}\right\|_{p}\right)<\infty$ and set

$$
\|\xi\|_{\mathbb{B}_{p}^{\Phi}}:=\left[\mathbb{E}|\xi|^{p}+\|\xi\|_{\Phi, p}^{p}\right]^{\frac{1}{p}} \quad \text { with } \quad\|\xi\|_{\Phi, p}:=\Phi\left(\varphi \rightarrow\left\|\xi-\xi^{\varphi}\right\|_{p}\right) .
$$

Proposition 4.13. For $p \in[1, \infty)$ the space $\mathbb{B}_{p}^{\Phi}$ is a Banach space.

Proof. The norm properties can be easily verified, we only verify the completeness. Assume a Cauchy sequence $\left(\xi_{n}\right)_{n \geq 1}$, we obtain by the completeness of $L_{p}$ a limit $\xi=\lim _{n} \xi_{n}$ in $L_{p}$. To show that the convergence takes place in $\mathbb{B}_{p}^{\Phi}$, let $\varepsilon>0$ and find $n_{\varepsilon} \geq 1$ such that for all $m, n \geq n_{\varepsilon}$ we have that 
$\left\|\xi_{n}-\xi_{m}\right\|_{p}^{p}+\Phi\left(F_{\xi_{n}-\xi_{m}, p}\right)^{p}<\varepsilon^{p}$ with $F_{\xi, p}(\varphi)=\left\|\xi-\xi^{\varphi}\right\|_{p}$. For all $m, n \geq 1$ we have that

$$
\begin{aligned}
& \left|F_{\xi_{n}-\xi_{m}, p}(\varphi)-F_{\xi_{n}-\xi, p}(\varphi)\right| \\
= & \left|\left\|\xi_{n}-\xi_{m}-\left(\xi_{n}-\xi_{m}\right)^{\varphi}\right\|_{p}-\left\|\xi_{n}-\xi-\left(\xi_{n}-\xi\right)^{\varphi}\right\|_{p}\right| \\
\leq & \left\|\xi-\xi_{m}-\left(\xi-\xi_{m}\right)^{\varphi}\right\|_{p} \\
\leq & 2\left\|\xi-\xi_{m}\right\|_{p},
\end{aligned}
$$

so that assumption (A4) implies for $n \geq n_{\varepsilon}$ that

$$
\left\|\xi_{n}-\xi\right\|_{p}^{p}+\Phi\left(F_{\xi_{n}-\xi, p}\right)^{p} \leq \lim _{m}\left\|\xi_{n}-\xi_{m}\right\|_{p}^{p}+\limsup _{m} \Phi\left(F_{\xi_{n}-\xi_{m}, p}\right)^{p} \leq 2 \varepsilon^{p} .
$$

\subsection{Connection to real interpolation}

Besov spaces (or fractional order Sobolev spaces) on the Wiener space were studied by various authors, see for example [88, [50, 14, Chapter 8.6], and [46. In this section we relate our definition of Besov spaces to the classical Gaussian Besov spaces obtained by the real interpolation method.

4.4.1. The isotropic case. In our intuition a functional $\Phi: C^{+}(\Delta) \rightarrow[0, \infty]$ is isotropic, provided that $\Phi$ depends on the constant functions in $C^{+}(\Delta)$ only.

Instead of giving a formal definition, we introduce a class of such functionals:

Definition 4.14. Let $\mu$ be a measure on $\mathcal{B}([0,1]), q \in[1, \infty]$, and let $K:[0,1] \rightarrow$ $[0, \infty)$ be measurable. Let $\varphi_{r}:(0, T] \rightarrow \mathbb{R}, r \in[0,1]$, denote the constant function $\varphi_{r} \equiv r$. For $F \in C^{+}(\Delta)$ we define

$$
\Phi^{(K, \mu, q)}(F):=\|K(\cdot) F(\varphi \cdot)\|_{L_{q}([0,1], \mu)} \in[0, \infty] .
$$

Recalling that $W^{\varphi_{r}}=\sqrt{1-r^{2}} W+r W^{\prime}$, we use for any $\xi \in \mathcal{L}_{0}(\Omega)$ the notation $\xi\left(\sqrt{1-r^{2}} W+r W^{\prime}\right):=\xi^{\varphi_{r}}$. For $q \in[1, \infty)$ Definition 4.14 yields to

$$
\|\xi\|_{\mathbb{B}_{p}^{\Phi}(K, \mu, q)}=\left[\|\xi\|_{p}^{p}+\left(\int_{0}^{1}\left[K(r)\left\|\xi(W)-\xi\left(\sqrt{1-r^{2}} W+r W^{\prime}\right)\right\|_{p}\right]^{q} d \mu(r)\right)^{\frac{p}{q}}\right]^{\frac{1}{p}} .
$$

LEMmA 4.15. The functional $\Phi^{(K, \mu, q)}$ satisfies the conditions (A1), (A2), (A3), and (A4).

Proof. From the definitions it follows that the map $r \mapsto F\left(\varphi_{r}\right)$ is continuous so that $\Phi^{(K, \mu, q)}(F)$ is well-defined. The assumptions (A1), (A2), and (A3) are immediate. To verify (A4), we assume $F_{n}, F: \Delta \rightarrow[0, \infty)$ to be continuous with

$$
\sup _{\varphi \in \Delta}\left|F_{n}(\varphi)-F(\varphi)\right| \rightarrow_{n} 0 .
$$

Then (A4) follows from the Fatou property of $L_{q}([0,1], \mu)$, because

$$
\begin{aligned}
\|K(\cdot) F(\varphi .)\|_{L_{q}([0,1], \mu)} & =\left\|\lim _{n} K(\cdot) F_{n}(\varphi .)\right\|_{L_{q}([0,1], \mu)} \\
& \leq \liminf _{n}\left\|K(\cdot) F_{n}(\varphi .)\right\|_{L_{q}([0,1], \mu)} .
\end{aligned}
$$


Theorem 4.16. For $\theta \in(0,1), q \in[1, \infty]$, and $p \in[2, \infty)$ one has that

$$
\|\xi\|_{\mathbb{B}_{p, q}^{\theta}} \sim_{c}\|\xi\|_{\mathbb{B}_{p}^{\Phi}(K, \mu, q)}
$$

with $d \mu(r):=\frac{r}{\sqrt{1-r^{2}}\left(1-\sqrt{1-r^{2}}\right)} \chi_{(0,1)}(r) d r$ and $K(r):=\left(1-\sqrt{1-r^{2}}\right)^{-\frac{\theta}{2}} \chi_{(0,1]}(r)$, and where $c \geq 1$ depends uniquely on $(p, q, \theta)$.

Proof. After a change of variables the assertion is equivalent to

$$
\|\xi\|_{\mathbb{B}_{p, q}^{\theta}} \sim_{c}\|\xi\|_{p}+\left\|(1-t)^{-\frac{\theta}{2}}\right\| \xi(W)-\xi\left(t W+\sqrt{1-t^{2}} W^{\prime}\right)\left\|_{p}\right\|_{L_{q}\left([0,1), \frac{d t}{1-t}\right)},
$$

which is the general form of 15 . Because the proof of 17$)$ in 46 relies on a finitedimensional argument, we still need to verify (19). First we remark the crucial fact, that the multiplicative constant in 15 does not depend on the dimension $n$. We use the proof of Proposition A.4 in the appendix with the supporting Hilbert space $H:=L_{2}^{\mathbb{R}^{d}}([0, T])$ and take the orthonormal basis from Section 3.1 We enumerate this tensor-basis and rename it to $\left(e_{i}\right)_{i=1}^{\infty}$. The $\sigma$-algebras $\mathcal{H}_{n}$ are defined as in the proof of Proposition A.4 We also set $\xi_{n}:=\mathbb{E}\left(\xi \mid \mathcal{H}_{n}\right)$ and observe the following:

(1) $\left|K\left(\xi, t ; L_{p}, \mathbb{D}_{1, p}\right)-K\left(\eta, t ; L_{p}, \mathbb{D}_{1, p}\right)\right| \leq\|\xi-\eta\|_{p}$ for $\xi, \eta \in L_{p}$.

(2) $\left\|\left(\xi_{0}\right)_{n}\right\|_{p} \leq\left\|\xi_{0}\right\|_{p}$ for $\xi_{0} \in L_{p}$.

(3) $\left\|\left(\xi_{1}\right)_{n}\right\|_{\mathbb{D}_{1, p}} \leq\left\|\xi_{1}\right\|_{\mathbb{D}_{1, p}}$ for $\xi_{1} \in \mathbb{D}_{1, p}$.

Assertions (2) and (3) give

$$
K\left(\xi_{n}, t ; L_{p}, \mathbb{D}_{1, p}\right) \leq K\left(\xi_{n+1}, t ; L_{p}, \mathbb{D}_{1, p}\right) \leq K\left(\xi, t ; L_{p}, \mathbb{D}_{1, p}\right) .
$$

Together with (1) we obtain

$$
K\left(\xi_{n}, t ; L_{p}, \mathbb{D}_{1, p}\right) \uparrow_{n} K\left(\xi, t ; L_{p}, \mathbb{D}_{1, p}\right),
$$

and finally $\left\|\xi_{n}\right\|_{\mathbb{B}_{p, q}^{\theta}} \uparrow_{n}\|\xi\|_{\mathbb{B}_{p, q}^{\theta}}$. On the other side, for $t \in[0,1)$ one has

$$
\left\|\xi_{n}(W)-\xi_{n}\left(t W+\sqrt{1-t^{2}} W^{\prime}\right)\right\|_{p} \leq\left\|\xi_{n+1}(W)-\xi_{n+1}\left(t W+\sqrt{1-t^{2}} W^{\prime}\right)\right\|_{p}
$$

which can be verified as follows: By Doob's factorization theorem we may write

$$
\xi_{n}=f_{n}\left(g_{e_{1}}, \ldots, g_{e_{n}}\right)
$$

where $f_{n}: \mathbb{R}^{n} \rightarrow \mathbb{R}$ is a Borel function. Then we get (note that $\left(g_{e_{k}}\right)_{k=1}^{\infty}$ are independent standard Gaussian random variables) for an independent copy $\left(g_{e_{k}}^{\prime}\right)_{k=1}^{\infty}$ that

$$
\begin{aligned}
&\left\|\xi_{n}(W)-\xi_{n}\left(t W+\sqrt{1-t^{2}} W^{\prime}\right)\right\|_{p}^{p} \\
&=\left\|f_{n}\left(g_{e_{1}}, \ldots, g_{e_{n}}\right)-f_{n}\left(t g_{e_{1}}+\sqrt{1-t^{2}} g_{e_{1}}^{\prime}, \ldots, t g_{e_{n}}+\sqrt{1-t^{2}} g_{e_{n}}^{\prime}\right)\right\|_{p}^{p} \\
&= \| \int_{\mathbb{R}} f_{n+1}\left(g_{e_{1}}, \ldots, g_{e_{n}}, \xi\right) d \gamma_{1}(\xi)-\int_{\mathbb{R}} \int_{\mathbb{R}} f_{n+1}\left(t g_{e_{1}}+\sqrt{1-t^{2}} g_{e_{1}}^{\prime}, \ldots,\right. \\
&\left.t g_{e_{n}}+\sqrt{1-t^{2}} g_{e_{n}}^{\prime}, t \xi+\sqrt{1-t^{2}} \xi^{\prime}\right) d \gamma_{1}(\xi) d \gamma_{1}\left(\xi^{\prime}\right) \|_{p}^{p} \\
& \leq \int_{\mathbb{R}} \int_{\mathbb{R}} \| f_{n+1}\left(g_{e_{1}}, \ldots, g_{e_{n}}, \xi\right)-f_{n+1}\left(t g_{e_{1}}+\sqrt{1-t^{2}} g_{e_{1}}^{\prime}, \ldots,\right. \\
&\left.t g_{e_{n}}+\sqrt{1-t^{2}} g_{e_{n}}^{\prime}, t \xi+\sqrt{1-t^{2}} \xi^{\prime}\right) \|_{p}^{p} d \gamma_{1}(\xi) d \gamma_{1}\left(\xi^{\prime}\right) \\
&=\left\|\xi_{n+1}(W)-\xi_{n+1}\left(t W+\sqrt{1-t^{2}} W^{\prime}\right)\right\|_{p}^{p} .
\end{aligned}
$$


This proves 20]. Moreover,

$$
\begin{aligned}
& \left\|\left[\xi_{n}(W)-\xi_{n}\left(t W+\sqrt{1-t^{2}} W^{\prime}\right)\right]-\left[\xi(W)-\xi\left(t W+\sqrt{1-t^{2}} W^{\prime}\right)\right]\right\|_{p} \\
\leq & 2\left\|\xi_{n}-\xi\right\|_{p} \rightarrow 0
\end{aligned}
$$

as $n \rightarrow \infty$. Together with 20 this yields to

$$
\left\|\xi_{n}(W)-\xi_{n}\left(t W+\sqrt{1-t^{2}} W^{\prime}\right)\right\|_{p} \uparrow_{n}\left\|\xi(W)-\xi\left(t W+\sqrt{1-t^{2}} W^{\prime}\right)\right\|_{p}
$$

and

$$
\begin{aligned}
&\left\|(1-t)^{-\frac{\theta}{2}}\right\| \xi_{n}(W)-\xi_{n}\left(t W+\sqrt{1-t^{2}} W^{\prime}\right)\left\|_{p}\right\|_{L_{q}\left([0,1), \frac{d t}{1-t}\right)} \\
& \uparrow_{n}\left\|(1-t)^{-\frac{\theta}{2}}\right\| \xi(W)-\xi\left(t W+\sqrt{1-t^{2}} W^{\prime}\right)\left\|_{p}\right\|_{L_{q}\left([0,1), \frac{d t}{1-t}\right)}
\end{aligned}
$$

which completes the proof because for $\xi_{n}$ the equivalence 19 was verified in 15 .

REMARK 4.17. There are other approaches to fractional smoothness on the Wiener space: One can use the Ornstein-Uhlenbeck semi-group which also gives a link to Mehler's formula (see [50, 14, Section 8.6], 11, Section 6.7]). For relations about this approach to Theorem 4.16 the reader is referred to [46, Remark 3.5]. Another approach can be found in [50, Theorem 13]. It uses an isotropic decoupling as we do, is formulated by means of the trace interpolation method (cf. [86. Section 1.8]), corresponds to the special choice $p=q$ in our setting, but yields to an alternative expression compared to Theorem 4.16 .

\subsubsection{An anisotropic example.}

Definition 4.18. For $0=r_{0}<r_{1}<\cdots r_{L}=T, \theta_{l} \in(0,1), q_{l} \in[1, \infty]$, and $F \in C^{+}(\Delta)$ we let

$$
\Phi_{r_{1}, \ldots, r_{L}}^{\left(\theta_{1}, q_{1}\right), \ldots,\left(\theta_{L}, q_{L}\right)}(F):=\sup _{l=1, \ldots, L}\left\|\left(r_{l}-t\right)^{-\theta_{l} / 2} F\left(\chi_{\left(t, r_{l}\right]}\right)\right\|_{L_{q_{l}}\left(\left[r_{l-1}, r_{l}\right), \frac{d t}{r_{l}-t}\right)} .
$$

This functional is admissible:

LEMma 4.19. The functional $\Phi_{r_{1}, \ldots, r_{L}}^{\left(\theta_{1}, q_{1}\right), \ldots,\left(\theta_{L}, q_{L}\right)}$ satisfies the conditions (A1), (A2), (A3), and (A4).

Proof. Because the proof is a copy of the proof of Lemma 4.15, we only check (A4). Assume $F_{n}, F \in C^{+}(\Delta)$ with $\sup _{\varphi \in \Delta}\left|F_{n}(\varphi)-F(\varphi)\right| \rightarrow_{n} 0$. Then, by the Fatou property of the spaces $L_{q_{l}}$,

$$
\begin{aligned}
& \sup _{l=1, \ldots, L}\left\|\left(r_{l}-t\right)^{-\theta_{l} / 2} F\left(\chi_{\left(t, r_{l}\right]}\right)\right\|_{L_{q_{l}}\left(\left[r_{l-1}, r_{l}\right), \frac{d t}{r_{l}-t}\right)} \\
= & \sup _{l=1, \ldots, L}\left\|\lim _{n}\left[\left(r_{l}-t\right)^{-\theta_{l} / 2} F_{n}\left(\chi_{\left(t, r_{l}\right]}\right)\right]\right\|_{L_{q_{l}}\left(\left[r_{l-1}, r_{l}\right), \frac{d t}{r_{l}-t}\right)} \\
\leq & \sup _{l=1, \ldots, L} \liminf _{n}\left\|\left[\left(r_{l}-t\right)^{-\theta_{l} / 2} F_{n}\left(\chi_{\left(t, r_{l}\right]}\right)\right]\right\|_{L_{q_{l}}\left(\left[r_{l-1}, r_{l}\right), \frac{d t}{r_{l}-t}\right)} \\
\leq & \liminf _{n} \sup _{l=1, \ldots, L}\left\|\left[\left(r_{l}-t\right)^{-\theta_{l} / 2} F_{n}\left(\chi_{\left(t, r_{l}\right]}\right)\right]\right\|_{L_{q_{l}}\left(\left[r_{l-1}, r_{l}\right), \frac{d t}{r_{l}-t}\right)},
\end{aligned}
$$


which proves (A4).

From (17) we obtain the following result about subspaces of $\mathbb{B}_{p}^{\Phi_{1}^{\left(\theta_{1}, q_{1}\right), \ldots, r_{L}}}$ isomorphic to $\mathbb{B}_{p, q_{l}}^{\theta_{l}}\left(\mathbb{R}^{d}, \gamma_{d}\right)$ :

Proposition 4.20. For a measurable function $f: \mathbb{R}^{d} \rightarrow \mathbb{R}, p \in[2, \infty), 0=r_{0}<$ $r_{1}<\cdots r_{L}=T, \theta_{l} \in(0,1), q_{l} \in[1, \infty]$, and $l=1, \ldots, L$ we have

$$
f \in \mathbb{B}_{p, q_{l}}^{\theta_{l}}\left(\mathbb{R}^{d}, \gamma_{d}\right) \quad \text { if and only if } f\left(\frac{W_{r_{l}}-W_{r_{l-1}}}{\sqrt{r_{l}-r_{l-1}}}\right) \in \mathbb{B}_{p}^{\Phi_{r_{1}, \ldots, r_{L}}^{\left.\left(\theta_{1}, q_{1}\right), \ldots, \theta_{L}, q_{L}\right)}} \text {. }
$$

Remark 4.21. Assume that $p \in[2, \infty), \theta_{1}, \ldots, \theta_{L} \in(0,1), 0=r_{0}<r_{1}<\cdots<$ $r_{L}=T$, and

$$
\xi \in \mathbb{B}_{p}^{\Phi_{r_{1}, \ldots, r_{L}}^{\left(\theta_{1}, \infty\right), \ldots,\left(\theta_{L}, \infty\right)} .}
$$

If we let

$$
\mathcal{G}_{a}^{b}:=\sigma\left(W_{t}: t \in[0, a]\right) \vee \sigma\left(W_{t}-W_{b}: t \in[b, T]\right) \quad \text { for } \quad 0 \leq a \leq b \leq T,
$$

then Lemma 4.23 below implies that there is a constant $c \geq 0$ such that

$$
\left\|\xi-\mathbb{E}\left(\xi \mid \mathcal{G}_{t}^{r_{l}}\right)\right\|_{p} \leq c\left(r_{l}-t\right)^{\frac{\theta_{l}}{2}} \quad \text { for } \quad t \in\left[r_{l-1}, r_{l}\right) \text { and } l=1, \ldots, L .
$$

In other words, the conditional expectations $\mathbb{E}\left(\xi \mid \mathcal{G}_{t}^{r_{l}}\right)$ converge to $\xi$ in $L_{p}$ with the speed $\left(r_{l}-t\right)^{\frac{\theta_{l}}{2}}$ as $t \uparrow r_{l}$. If $\theta_{l}<1$, then one can interpret this as a singularity of order $1-\theta_{l}$ at $r_{l}$ because $\left(r_{l}-t\right)^{\frac{1}{2}}$ would be the speed for $\xi=W_{T}$. The concept from 21] was applied in 40] in the context of BSDEs to obtain path-dependent variational estimates. The setting of BSDEs, where we have a backward equation with a pre-given terminal condition, did require the consideration of $\xi-\mathbb{E}\left(\xi \mid \mathcal{G}_{t}^{r_{l}}\right)$ rather than that one of $\mathbb{E}\left(\xi \mid \mathcal{F}_{r_{l}}\right)-\mathbb{E}\left(\xi \mid \mathcal{F}_{t}\right)$, which could have been a first attempt. The fact that in 40 the $\theta_{l}$ are allowed to be different from each other is one reason to extend the isotropic spaces $\mathbb{B}_{p, q}^{\theta}$ from 14 to the spaces $\mathbb{B}_{p}^{\Phi}$ that might be anisotropic.

\subsection{The space $\mathbb{B}_{p}^{\Phi_{2}}$}

In this section we study the space $\mathbb{B}_{p}^{\Phi_{2}}$, where the functional $\Phi_{2}: C^{+}(\Delta) \rightarrow[0, \infty]$ is given by

$$
\Phi_{2}(F):=\sup _{0 \leq s<t \leq T} \frac{F\left(\chi_{(s, t]}\right)}{\sqrt{t-s}} .
$$

To describe these spaces we let, for $p \in(0, \infty)$ and a measurable $\lambda:[0, T] \times \Omega \rightarrow \mathbb{R}^{d}$ with $\mathbb{E} \int_{0}^{T}\left|\lambda_{s}\right|^{2} d s<\infty$,

$$
\begin{aligned}
\|\lambda\|_{L_{\infty}\left([0, T] ; L_{p}(\Omega)\right)} & :=\operatorname{esssup}_{s \in[0, T]}\left|\left\|\lambda_{s} \mid\right\|_{p}\right. \\
\|\lambda\|_{L_{p}^{*}\left(\Omega ; L_{2}([0, T])\right)} & :=\sup _{0 \leq a<b \leq T}\left\|\left(\frac{1}{b-a} \int_{a}^{b}\left|\lambda_{s}\right|^{2} d s\right)^{\frac{1}{2}}\right\|_{p} .
\end{aligned}
$$

To shorten the notation we also use $\left\|\lambda_{s}\right\|_{p}=\left\|\left|\lambda_{s}\right|\right\|_{p}$. We already introduced $L_{q}^{X}(\Omega)$ when $X$ is a separable Banach space. Above we use a different notation as we want 
to avoid a discussion about the separability of $L_{p}(\Omega)$, which is not needed here. By the Lebesgue differentiation theorem (cf. Lemma A.3 below) one has that

$$
\begin{aligned}
\|\lambda\|_{L_{\infty}\left([0, T] ; L_{2}(\Omega)\right)} & =\|\lambda\|_{L_{2}^{*}\left(\Omega ; L_{2}([0, T])\right)}, \\
\|\lambda\|_{L_{p}^{*}\left(\Omega ; L_{2}([0, T])\right)} & \leq\|\lambda\|_{L_{\infty}\left([0, T] ; L_{p}(\Omega)\right)} \text { for } 2 \leq p<\infty, \\
\|\lambda\|_{L_{\infty}\left([0, T] ; L_{p}(\Omega)\right)} & \leq\|\lambda\|_{L_{p}^{*}\left(\Omega ; L_{2}([0, T])\right)} \text { for } 0<p \leq 2 .
\end{aligned}
$$

The next theorem, the main result of this section, is motivated as follows: If $\xi \in \mathbb{B}_{2}^{\Phi_{2}}$, then $\xi \in \mathbb{D}_{1,2}$ and the quantity $\|\xi\|_{\Phi_{2}, p}$ enables us to access the Malliavin derivative of $\xi$ without its explicit computation. As in Corollary 1.6 of Section 1.2 announced, this can be exploited in the context of BSDEs to obtain the differentiability of the $Y$-process without differentiating the BSDE.

TheOREM 4.22. One has that $\mathbb{B}_{2}^{\Phi_{2}} \subseteq \mathbb{D}_{1,2}$ and the following assertions hold true:

(1) For $p \in[2, \infty)$ and $\xi \in \mathbb{D}_{1,2} \cap L_{p}$ one has

$$
\|\xi\|_{\Phi_{2}, p} \sim_{c_{\underline{4.22\}}(1), p}}\|D \xi\|_{L_{p}^{*}\left(\Omega ; L_{2}([0, T])\right)}
$$

where $c \sqrt{4.22}(1), p \geq 1$ depends on $p$ only.

(2) For $p \in(1,2)$ and $\xi \in \mathbb{D}_{1,2}$ one has

$$
\frac{1}{c \sqrt{4.22)(2), p}}\|D \xi\|_{L_{\infty}\left([0, T] ; L_{p}(\Omega)\right)} \leq\|\xi\|_{\Phi_{2}, p} \leq c \sqrt{\underline{4.22}(2), p}\|D \xi\|_{L_{p}^{*}\left(\Omega ; L_{2}([0, T])\right)},
$$

where $c \sqrt{4.22}(2), p \geq 1$ depends on $p$ only.

(3) There is a $\xi \in \mathbb{D}_{1,2}$ such that for all $p \in[1, \infty)$ one has $\xi \in L_{p}(\Omega)$, D $\xi \in$ $L_{p}\left(\Omega ; L_{2}([0, T])\right)$, and $\xi \notin \mathbb{B}_{p}^{\Phi_{2}}$.

In the inequalities of the theorem above the expressions might be infinite. For the case $p \in(1,2)$ the result is still incomplete. However, if one is interested in good moment estimates, then the case $p \in[2, \infty)$ seems to be of more interest than the case $p \in(1,2)$. To prove Theorem 4.22 we let

$$
\mathcal{G}_{a}^{b}:=\sigma\left(W_{t}: t \in[0, a]\right) \vee \sigma\left(W_{t}-W_{b}: t \in[b, T]\right)
$$

for $0 \leq a \leq b \leq T$ considered as $\sigma$-algebra in $(\Omega, \mathcal{F}, \mathbb{P})$.

Lemma 4.23. For $p \in[1, \infty], \xi=\left(\xi_{1}, \ldots, \xi_{m}\right)$ with $\xi_{1}, \ldots, \xi_{m} \in \mathcal{L}_{p}(\Omega, \mathcal{F}, \mathbb{P})$, a norm $\|\cdot\|$ on $\mathbb{R}^{m}$, and $0 \leq s<t \leq T$ one has

$$
\frac{1}{2}\|\| \xi-\xi^{(s, t]}\|\|_{p} \leq\|\| \xi-\mathbb{E}^{\mathcal{G}_{s}^{t} \xi \|}\left\|_{p} \leq\right\|\left\|\xi-\xi^{(s, t]}\right\| \|_{p},
$$

where in the first and last expression $\xi_{1}, \ldots, \xi_{m}$ are extended to $\bar{\Omega}$ according to Convention 4.5 and the conditional expectation is taken coordinate-wise.

Proof. By $p \rightarrow \infty$ it is sufficient to show the assertion for $p \in[1, \infty)$. Assuming $p \in[1, \infty)$ it is sufficient to consider $\xi=\left(\xi_{1}, \ldots, \xi_{m}\right)$ of the form

$$
\xi=f\left(W_{t_{1}}-W_{t_{0}}, \ldots, W_{t_{n}}-W_{t_{n-1}}\right)
$$

where $0 \leq t_{0}<\cdots t_{n} \leq T$ and $f: \mathbb{R}^{n d} \rightarrow \mathbb{R}^{m}$ is continuous and bounded. W.l.o.g. we can assume that $s$ and $t$ belong to the partition points. Then

$$
\|\| \xi-\xi^{(s, t]}\|\|_{p} \leq\|\| \xi-\mathbb{E}^{\mathcal{G}_{s}^{t}} \xi\|\|_{p}+\left\|\widetilde{\mathbb{E}^{\mathcal{G}_{s}^{t}} \xi}-\xi^{(s, t]}\right\|\left\|_{p}=2\right\| \| \xi-\mathbb{E}^{\mathcal{G}_{s}^{t} \xi\|\|_{p}}
$$


and

$$
\|\| \xi-\mathbb{E}^{\mathcal{G}_{s}^{t}} \xi\|\|_{p}=\|\| \xi-\mathbb{E}\left(\xi^{(s, t]} \mid \sigma\left(W_{r}: r \in[0, T]\right)\right)\|\|_{p} \leq\|\| \xi-\xi^{(s, t]}\|\|_{p} .
$$

Proof of Theorem 4.22. (3) For $l \geq 1$ we take disjoint intervals $\left(s_{l}, t_{l}\right] \subseteq$ $(0, T]$ with $t_{l}-s_{l}=T 4^{-l}$ and $t_{l}<s_{l+1}$. Define

$$
A_{l}:=l \cos \left(W_{s_{l}, 1}\right)\left(W_{t_{l}, 1}-W_{s_{l}, 1}\right) \quad \text { and } \quad \xi:=\sum_{l=1}^{\infty} A_{l} .
$$

The sum converges in any $L_{p}, p \in[1, \infty)$, as

$$
\sum_{l=1}^{\infty}\left\|l \cos \left(W_{s_{l}, 1}\right)\left(W_{t_{l}, 1}-W_{s_{l}, 1}\right)\right\|_{p} \leq c_{p} \sum_{l=1}^{\infty} l \sqrt{t_{l}-s_{l}}<\infty
$$

where $c_{p}:=\|g\|_{p}$ with $g \sim N(0,1)$. Moreover,

$$
D A_{l}=l\left[\cos \left(W_{s_{l}, 1}\right) \chi_{\left(s_{l}, t_{l}\right]}-\sin \left(W_{s_{l}, 1}\right)\left(W_{t_{l}, 1}-W_{s_{l}, 1}\right) \chi_{(0, s]}\right]
$$

so that

$$
\left\|D A_{l}\right\|_{L_{q}^{L_{2}((0, T])}} \leq l\left[\sqrt{t_{l}-s_{l}}+\sqrt{T} c_{q} \sqrt{t_{l}-s_{l}}\right]
$$

for $q \in[2, \infty)$. This implies $\xi \in \mathbb{D}_{1,2}$ and $D \xi \in L_{q}^{L_{2}([0, T])}(\Omega)$. On the other hand,

$$
\begin{aligned}
& \frac{\left\|A_{L}-A_{L}^{\left(s_{L}, t_{L}\right.}\right\|_{p}}{\sqrt{t_{L}-s_{L}}} \\
& \quad=L \frac{\left\|\cos \left(W_{s_{L}, 1}\right)\left(W_{t_{L}, 1}-W_{s_{L}, 1}-\left(W^{\prime}\right)_{t_{L}, 1}+\left(W^{\prime}\right)_{s_{L}, 1}\right)\right\|_{p}}{\sqrt{t_{L}-s_{L}}} \\
& \quad \geq L \sqrt{2} c_{p}\left\|\cos \left(W_{s_{L}, 1}\right)\right\|_{p} \geq L \kappa_{p}
\end{aligned}
$$

where $\kappa_{p}:=\sqrt{2} c_{p} \inf _{s \in[0, T]}\left\|\cos \left(W_{s, 1}\right)\right\|_{p}>0$. For each $L \geq 1$ this implies

$$
\frac{\left\|\xi-\xi^{\left(s_{L}, t_{L}\right]}\right\|_{p}}{\sqrt{t_{L}-s_{L}}} \geq \frac{\left\|\sum_{l=1}^{L}\left(A_{l}-A_{l}^{\left(s_{L}, t_{L}\right]}\right)\right\|_{p}}{\sqrt{t_{L}-s_{L}}}=\frac{\left\|A_{L}-A_{L}^{\left(s_{L}, t_{L}\right]}\right\|_{p}}{\sqrt{t_{L}-s_{L}}} \geq \kappa_{p} L
$$

and therefore $\xi \notin \mathbb{B}_{p}^{\Phi_{2}}$.

(1) and (2) Step (a): We prove $\mathbb{B}_{2}^{\Phi_{2}} \subseteq \mathbb{D}_{1,2}$. Let $0 \leq a<b \leq T$, and define for $n \geq 1$ the set

$$
D_{n}(a, b):=\left\{\left(t_{1}, \ldots, t_{n}\right) \in(0, T]^{n}: \text { there is a } k \text { such that } t_{k} \in(a, b]\right\} .
$$

Assume $\xi \in L_{2}$ with chaos decomposition

$$
\xi=\sum_{n=0}^{\infty} I_{n}\left(f_{n}\right)
$$

with symmetric $f_{n}:((0, T] \times\{1, \ldots, d\})^{n} \rightarrow \mathbb{R}$, cf. [70, Example 1.1.2]. By Lemma 4.23 the condition $\xi \in \mathbb{B}_{2}^{\Phi_{2}}$ is equivalent to the condition

$$
\sum_{n=1}^{\infty} n !\left\|f_{n} \chi_{D_{n}(a, b)}\right\|_{L_{2}^{n}}^{2} \leq c^{2}(b-a)
$$


for all $0 \leq a<b \leq T$, where $L_{2}^{n}:=L_{2}\left(((0, T] \times\{1, \ldots, d\})^{n}, \mu^{n}\right)$ with $\mu:=\lambda \otimes$ $\left(\sum_{i=1}^{d} \delta_{\{i\}}\right)$ and $\lambda$ being the Lebesgue measure. For $L \geq 1, l=1, \ldots, 2^{L}$, and $n \geq 1$ let

so that

$$
D_{n}^{l, L}:=D_{n}\left(T \frac{l-1}{2^{L}}, T \frac{l}{2^{L}}\right),
$$

$$
\sum_{n=1}^{\infty} n !\left\|f_{n} \chi_{D_{n}^{l, L}}\right\|_{L_{2}^{n}}^{2} \leq c^{2} 2^{-L}
$$

Summing up over $l$ gives for all $N \geq 1$ that

$$
\sum_{n=1}^{N} n ! \sum_{l=1}^{2^{L}}\left\|f_{n} \chi_{D_{n}^{l, L}}\right\|_{L_{2}^{n}}^{2} \leq c^{2}
$$

Let $\Delta_{n}^{L}$ be the union of all dyadic half-open cubes

$$
\left(T \frac{l_{1}-1}{2^{L}}, T \frac{l_{1}}{2^{L}}\right] \times \cdots \times\left(T \frac{l_{n}-1}{2^{L}}, T \frac{l_{n}}{2^{L}}\right]
$$

with $l_{1}, \ldots, l_{n} \in\left\{1, \ldots, 2^{L}\right\}$ pair-wise distinct. Then

$$
\Delta_{n}^{L} \subseteq \bigcup_{l=1}^{2^{L}} D_{n}^{l, L}
$$

and

$$
\operatorname{card}\left\{l \in\left\{1, \ldots, 2^{L}\right\}:\left(t_{1}, \ldots, t_{n}\right) \in D_{n}^{l, L}\right\}=n \quad \text { for all } \quad\left(t_{1}, \ldots, t_{n}\right) \in \Delta_{n}^{L} .
$$

Now we get that

$$
\sum_{n=1}^{N} n ! n\left\|f_{n} \chi_{\Delta_{n}^{L}}\right\|_{L_{2}^{n}}^{2} \leq \sum_{n=1}^{N} n ! \sum_{l=1}^{2^{L}}\left\|f_{n} \chi_{D_{n}^{l, L}}\right\|_{L_{2}^{n}}^{2} \leq c^{2} .
$$

By $L \rightarrow \infty$ it follows that

Finally, $N \rightarrow \infty$ gives $\xi \in \mathbb{D}_{1,2}$.

$$
\sum_{n=1}^{N} n ! n\left\|f_{n}\right\|_{L_{2}^{n}}^{2} \leq c^{2}
$$

Step (b): Let $\xi \in \mathbb{D}_{1,2}$ with chaos expansion $\xi=\sum_{n=0}^{\infty} I_{n}\left(f_{n}\right)$ obtained by sym${\overline{\text { metric } f_{n}}}_{n}$ and fix $b \in(0, T]$. Consider the processes $\left(\mu_{t}^{b}(i)\right)_{t \in[0, b]}$ from Lemma A.10. so that for $p \in(1, \infty)$ and $a \in[0, b)$ we have that

$$
\left\|\xi-\xi^{(a, b]}\right\|_{p} \sim_{2}\left\|\xi-\mathbb{E}\left(\xi \mid \mathcal{G}_{a}^{b}\right)\right\|_{p} \sim_{c[\text { [.10] }}\left\|\left(\int_{a}^{b}\left|\mu_{s}^{b}\right|^{2} d s\right)^{\frac{1}{2}}\right\|_{p}
$$

where Lemma 4.23 is exploited in the first equivalence. For $s \in[a, b]$ and $n \geq 0$ let $t_{k}^{n}:=a+\left(k / 2^{n}\right)(b-a)$ for $k=0, \ldots, 2^{n}$ and

$$
b_{n}(s):=\inf \left\{t_{k}^{n}: s \leq t_{k}^{n}, k=0, \ldots, 2^{n}\right\} .
$$

Using 22 and Lemma 4.23 we get that

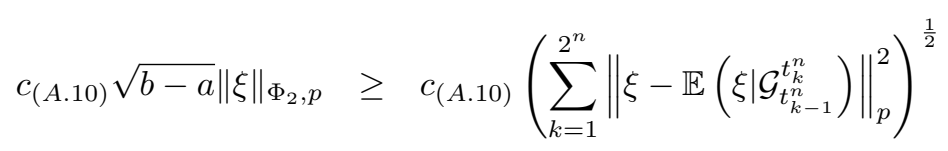




$$
\begin{aligned}
& \text { 4.5. THE SPACE } \mathbb{B}_{p}^{\Phi_{2}} \\
& \geq\left(\sum_{k=1}^{2^{n}}\left\|\left(\int_{\left(t_{k-1}^{n}, t_{k}^{n}\right]}\left|\mu_{s}^{b_{n}(s)}\right|^{2} d s\right)^{\frac{1}{2}}\right\|_{p}^{2}\right)^{\frac{1}{2}} .
\end{aligned}
$$

For $p \in[2, \infty)$ we continue by Fatou's lemma to

$$
\begin{aligned}
c\left[\sqrt{\text { A.10 }} \sqrt{b-a}\|\xi\|_{\Phi_{2}, p}\right. & \geq \liminf _{n}\left(\sum_{k=1}^{2^{n}}\left\|\left(\int_{\left(t_{k-1}^{n}, t_{k}^{n}\right]}\left|\mu_{s}^{b_{n}(s)}\right|^{2} d s\right)^{\frac{1}{2}}\right\|^{2}\right)_{p}^{\frac{1}{2}} \\
& \geq \liminf _{n}\left\|\left(\sum_{k=1}^{2^{n}} \int_{\left(t_{k-1}^{n}, t_{k}^{n}\right]}\left|\mu_{s}^{b_{n}(s)}\right|^{2} d s\right)^{\frac{1}{2}}\right\|_{p} \\
& =\liminf _{n}\left\|\left(\int_{(a, b]}\left|\mu_{s}^{b_{n}(s)}\right|^{2} d s\right)^{\frac{1}{2}}\right\|_{p} \\
& \geq\left\|\liminf _{n}\left(\int_{(a, b]}\left|\mu_{s}^{b_{n}(s)}\right|^{2} d s\right)^{\frac{1}{2}}\right\|_{p} \\
& \geq\left\|\left(\int_{(a, b]} \liminf _{n}\left|\mu_{s}^{b_{n}(s)}\right|^{2} d s\right)^{\frac{1}{2}}\right\|_{p} .
\end{aligned}
$$

For $p \in(1,2)$ we get

$$
\begin{aligned}
& c_{[A .10]} \sqrt{b-a}\|\xi\|_{\Phi_{2}, p} \\
\geq & \liminf _{n}\left(\sum_{k=1}^{2^{n}}\left\|\left(\int_{\left(t_{k-1}^{n}, t_{k}^{n}\right]}\left|\mu_{s}^{b_{n}(s)}\right|^{2} d s\right)^{\frac{1}{2}}\right\|_{p}^{2}\right)^{\frac{1}{2}} \\
\geq & \liminf _{n}\left(\int_{(a, b]}\left\|\mu_{s}^{b_{n}(s)}\right\|_{p}^{2} d s\right)^{\frac{1}{2}} \\
\geq & \left(\int_{(a, b]}\left\|\liminf _{n}\left|\mu_{s}^{b_{n}(s)}\right|\right\|_{p}^{2} d s\right)^{\frac{1}{2}} .
\end{aligned}
$$

Summarizing, this yields to

$$
\|\xi\|_{\Phi_{2}, p} \geq \frac{1}{c \sqrt{A .10}}\left\{\begin{array}{l}
\left\|\left(\frac{1}{b-a} \int_{(a, b]} \liminf _{n}\left|\mu_{s}^{b_{n}(s)}\right|^{2} d s\right)^{\frac{1}{2}}\right\|_{p}: p \in[2, \infty) \\
\left(\frac{1}{b-a} \int_{(a, b]}\left\|\liminf _{n}\left|\mu_{s}^{b_{n}(s)}\right|\right\|_{p}^{2} d s\right)^{\frac{1}{2}}: p \in(1,2)
\end{array} .\right.
$$

Now we observe that

$$
\begin{aligned}
& \lim _{n} \int_{(a, b]} \mathbb{E}\left|\mu_{s}^{b_{n}(s)}(i)-D(s, i) \xi\right|^{2} d s \\
= & \lim _{n} \int_{(a, b]} \sum_{k=1}^{\infty} k^{2}(k-1) !\left\|f_{k}((s, i), \cdot)\left(\chi_{\left((0, s] \cup\left(b_{n}(s), T\right]\right)^{k-1}}-1\right)\right\|_{L_{2}^{k-1}}^{2} d s
\end{aligned}
$$


$=0$

which follows by dominated convergence since

$$
\begin{aligned}
& \int_{(a, b]} \sum_{k=1}^{\infty} k^{2}(k-1) !\left\|f_{k}((s, i), \cdot)\left(\chi_{\left((0, s] \cup\left(b_{n}(s), T\right]\right)^{k-1}}-1\right)\right\|_{L_{2}^{k-1}}^{2} d s \\
\leq & \int_{(0,1]} \sum_{k=1}^{\infty} k^{2}(k-1) !\left\|f_{k}((s, i), \cdot)\right\|_{L_{2}^{k-1}}^{2} d s \\
\leq & \|\xi\|_{\mathbb{D}_{1,2}}^{2} .
\end{aligned}
$$

Hence there is a sub-sequence $\left(n_{l}\right)_{l=1}^{\infty}$ such that $\lim _{l} \mu_{s}^{b_{n_{l}}(s)}=D(s, \cdot) \xi \lambda \otimes \mathbb{P}$ a.e. on $(a, b] \times \Omega$. Observing that $(23)$ holds for the sub-sequence $\left(n_{l}\right)_{l=1}^{\infty}$ without modification as well, the desired lower bounds of $\|\xi\|_{\Phi_{2}, p}$ follow.

Step (c): We verify the upper bounds of (1) and (2). Let us first assume that $\xi$ is smooth like in Proposition A.4 i.e. by using the Haar system as orthogonal basis we may assume that

$$
\xi=f\left(W_{t_{1}}-W_{t_{0}}, \ldots, W_{t_{n}}-W_{t_{n-1}}\right)
$$

where $0=t_{0}<\cdots<t_{n}=T$ and $f \in C^{\infty}\left(\mathbb{R}^{n d}\right)$ is bounded with bounded derivatives of all orders (the bounds for the derivatives can depend on their order). By a possible redefinition of $f$ we can assume w.l.o.g. that $a=t_{k}<t_{l}=b$. We get

$$
D \xi=\sum_{i=1}^{n} \nabla_{i} f\left(W_{t_{1}}-W_{t_{0}}, \ldots, W_{t_{n}}-W_{t_{n-1}}\right) \chi_{\left(t_{i-1}, t_{i}\right]},
$$

where $\nabla_{i}$ is the $d$-dimensional gradient acting on the $i$-block of variables. We fix $\xi_{1}, \ldots, \xi_{k}, \xi_{l+1}, \ldots, \xi_{n} \in \mathbb{R}^{d}$ and let

$$
\begin{aligned}
f_{\xi}\left(\eta_{k+1}, \ldots, \eta_{l}\right) & :=f\left(\xi_{1}, \ldots, \xi_{k}, \eta_{k+1}, \ldots, \eta_{l}, \xi_{l+1}, \ldots, \xi_{n}\right), \\
f_{\xi}^{0}\left(\eta_{k+1}, \ldots, \eta_{l}\right) & :=f_{\xi}\left(\eta_{k+1} \sqrt{\delta_{k+1}}, \ldots, \eta_{l} \sqrt{\delta_{l}}\right)
\end{aligned}
$$

for $\delta_{i}:=t_{i}-t_{i-1}$. Moreover, we note that

$$
\begin{array}{r}
\left\|\xi-\mathbb{E}\left(\xi \mid \mathcal{G}_{a}^{b}\right)\right\|_{p}=\| f\left(W_{t_{1}}-W_{t_{0}}, \ldots, W_{t_{n}}-W_{t_{n-1}}\right)- \\
\mathbb{E}_{k+1}^{l} f\left(W_{t_{1}}-W_{t_{0}}, \ldots, W_{t_{n}}-W_{t_{n-1}}\right) \|_{p},
\end{array}
$$

where $\mathbb{E}_{k+1}^{l}$ is the expected value with respect to the increments

$$
\left(W_{t_{k+1}}-W_{t_{k}}, \ldots, W_{t_{l}}-W_{t_{l-1}}\right) .
$$

Applying Lemma A.7 yields to

$$
\begin{aligned}
& \left\|f_{\xi}(W)-\mathbb{E} f_{\xi}(W)\right\|_{p} \\
= & \| f_{\xi}^{0}\left(\frac{W_{t_{k+1}}-W_{t_{k}}}{\sqrt{\delta_{k+1}}}, \ldots, \frac{W_{t_{l}}-W_{t_{l-1}}}{\sqrt{\delta_{l}}}\right) \\
& -\mathbb{E} f_{\xi}^{0}\left(\frac{W_{t_{k+1}}-W_{t_{k}}}{\sqrt{\delta_{k+1}}}, \ldots, \frac{W_{t_{l}}-W_{t_{l-1}}}{\sqrt{\delta_{l}}}\right) \|_{p} \\
\leq & c \sqrt{A .7]}\left\|\left(\sum_{i=1}^{l-k}\left|\nabla_{i} f_{\xi}^{0}\left(\frac{W_{t_{k+1}}-W_{t_{k}}}{\sqrt{\delta_{k+1}}}, \ldots, \frac{W_{t_{l}}-W_{t_{l-1}}}{\sqrt{\delta_{l}}}\right)\right|^{2}\right)^{\frac{1}{2}}\right\|_{p}
\end{aligned}
$$




$$
\begin{aligned}
=c \sqrt{A .7]} \|\left(\sum_{i=k+1}^{l} \delta_{i} \mid \nabla_{i} f\left(\xi_{1}, \ldots, \xi_{k}, W_{t_{k+1}}-W_{t_{k}},\right.\right. \\
\left.\left.\ldots, W_{t_{l}}-W_{t_{l-1}}, \xi_{l+1}, \ldots, \xi_{n}\right)\left.\right|^{2}\right)^{\frac{1}{2}} \|_{p}
\end{aligned}
$$

and

$$
\begin{aligned}
& \left\|\xi-\mathbb{E}\left(\xi \mid \mathcal{G}_{a}^{b}\right)\right\|_{p} \\
& =\| f\left(W_{t_{1}}-W_{t_{0}}, \ldots, W_{t_{n}}-W_{t_{n-1}}\right)- \\
& \mathbb{E}_{k+1}^{l} f\left(W_{t_{1}}-W_{t_{0}}, \ldots, W_{t_{n}}-W_{t_{n-1}}\right) \|_{p} \\
& \leq c_{\underline{\text { A.7 }}}\left\|\left(\sum_{i=k+1}^{l} \delta_{i}\left|\nabla_{i} f(W)\right|^{2}\right)^{\frac{1}{2}}\right\|_{p} \\
& =c \sqrt{A .7)}\left\|\left(\sum_{i=k+1}^{l} \int_{\left(t_{i-1}, t_{i}\right]}\left|\nabla_{i} f(W)\right|^{2} d s\right)^{\frac{1}{2}}\right\|_{p} \\
& =c \sqrt{A .7]}\left\|\left(\int_{(a, b]}\left|D_{s} \xi\right|^{2} d s\right)^{\frac{1}{2}}\right\|_{p} .
\end{aligned}
$$

Now we assume the general case and let $q:=p \vee 2$. Our assumptions in (1) and (2) and under the assumption that the right-hand sides in (1) and (2) are finite, we have that $\xi \in \mathbb{D}_{1,2} \cap L_{q}$ and $D \xi \in L_{q}^{L_{2}([0, T])}(\Omega)$. Using Proposition A.4 we find smooth $\xi_{n}$ such that

$$
\xi_{n} \rightarrow \xi \quad \text { in } \quad L_{q} \quad \text { and } \quad D \xi_{n} \rightarrow D \xi \quad \text { in } \quad L_{q}^{L_{2}([0, T])}(\Omega) .
$$

Therefore by approximation,

$$
\left\|\xi-\mathbb{E}\left(\xi \mid \mathcal{G}_{a}^{b}\right)\right\|_{p} \leq c_{\underline{A .7]}}\left\|\left(\int_{(a, b]}\left|D_{s} \xi\right|^{2} d s\right)^{\frac{1}{2}}\right\|_{p}
$$

under the assumptions (1) and (2). Dividing by $\sqrt{b-a}$ and taking the supremum over $0 \leq a<b \leq T$ gives the upper bound of $\|\xi\|_{\Phi_{2}, p}$.

\subsection{An embedding theorem for functionals of bounded variation}

We extend the approach from Section 4.5 to the functionals $\Phi_{r}: C^{+}(\Delta) \rightarrow[0, \infty]$, $r \in[2, \infty)$, given by

$$
\Phi_{r}(F):=\sup _{0 \leq s<t \leq T} \frac{F\left(\chi_{(s, t]}\right)}{(t-s)^{\frac{1}{r}}}
$$

Definition 4.24. A Borel function $g: \mathbb{R} \rightarrow \mathbb{R}$ is of bounded variation provided that

$$
V(g):=\sup _{-\infty<x_{0}<\cdots<x_{n}<\infty} \sum_{k=1}^{n}\left|g\left(x_{k}\right)-g\left(x_{k-1}\right)\right|<\infty .
$$


It follows from the definition that a function of bounded variation is bounded. A typical example is $g=\chi_{[K, \infty)}$ where $V\left(\chi_{[K, \infty)}\right)=1$. Now we get the following embedding:

TheOREM 4.25. Let $r \in[2, \infty), p \in[1, \infty), \xi \in \mathbb{B}_{p}^{\Phi_{r}}$ and $g: \mathbb{R} \rightarrow \mathbb{R}$ be of bounded variation. Assume that the law of $\xi$ has a bounded density $\rho$. Then, for all $q \in$ $[1, \infty)$

$$
g(\xi) \in \mathbb{B}_{q}^{\Phi_{\tilde{r}}} \quad \text { with } \quad \tilde{r}:=\frac{p+1}{p} q r .
$$

Proof. We use [4, Theorem 2.4] and get that

$$
\begin{aligned}
\left(\mathbb{E}\left|g(\xi)-(g(\xi))^{(s, t]}\right|^{q}\right)^{\frac{1}{q}} & =\left(\mathbb{E}\left|g(\xi)-\left(g\left(\xi^{(s, t]}\right)\right)\right|^{q}\right)^{\frac{1}{q}} \\
& \leq 3^{\frac{q+1}{q}}\left(\sup _{x \in \mathbb{R}} \rho(x)\right)^{\frac{1}{q} \frac{p}{p+1}} V(g)\left\|\xi-\xi^{(s, t]}\right\|_{p}^{\frac{1}{q} \frac{p}{p+1}} .
\end{aligned}
$$

Dividing by $(t-s)^{\frac{1}{r} \frac{1}{q} \frac{p}{p+1}}$ gives the assertion.

In view of Example 4.27 the following limiting case is important:

Corollary 4.26. If $r \in[2, \infty), \xi \in \bigcap_{p \in[1, \infty)} \mathbb{B}_{p}^{\Phi_{r}}$ has a bounded density, and if $g: \mathbb{R} \rightarrow \mathbb{R}$ is of bounded variation, then

$$
g(\xi) \in \bigcap_{q \in[1, \infty)} \bigcap_{\tilde{r} \in(q r, \infty)} \mathbb{B}_{q}^{\Phi_{\tilde{r}}} .
$$

\subsection{Examples}

4.7.1. Forward diffusions. The Malliavin differentiability of diffusions is well investigated, see for example [70. So the following is expected:

EXAMPLE 4.27. Let

$$
X_{t}=x_{0}+\int_{0}^{t} \sigma\left(s, X_{s}\right) d W_{s}+\int_{0}^{t} b\left(s, X_{s}\right) d s
$$

where $\sigma:[0, T] \times \mathbb{R}^{d} \rightarrow \mathbb{R}^{d} \times \mathbb{R}^{d}$ and $b:[0, T] \times \mathbb{R}^{d} \rightarrow \mathbb{R}^{d}$ are bounded and continuous, and satisfy

$$
|\sigma(t, x)-\sigma(t, y)|+|b(t, x)-b(t, y)| \leq L|x-y| \text { for some } \quad L \geq 0 .
$$

By the proof of [40. Theorem 3] this implies for $p \in[2, \infty)$ that

$$
\left\|X_{T}^{\varphi}-X_{T}\right\|_{p} \leq c\left(\int_{0}^{T} \varphi(r)^{2} d r\right)^{\frac{1}{2}}
$$

with $c=c(p, T, b, \sigma)>0$. In particular, for $X_{T}=\left(X_{T}^{1}, \ldots, X_{T}^{d}\right)$,

$$
X_{T}^{i} \in \bigcap_{p \in(0, \infty)} \mathbb{B}_{p}^{\Phi_{2}}
$$

which follows by using $\varphi=\chi_{(s, t]}$ for $0 \leq s<t \leq T$. 
4.7.2. Local time. One can look at the fractional smoothness of local times $\left(L_{t}^{\alpha}\right)_{t \in(0, T], \alpha \in \mathbb{R}}$ of a one-dimensional Brownian motion from different points of view: In [19, 18] the smoothness with respect to the state variable $\alpha$ is under consideration, whereas in [72, 1 (with a generalization in [89]) the smoothness in $\omega$ for fixed $(t, \alpha)$ is investigated within the interpolation spaces generated by the OrnsteinUhlenbeck operator. Our result complements [1, Theorem 1]. The smoothness obtained in $\mathbf{1}$ is strictly smaller than $1 / 2$. In Theorem 4.28 and Corollary 4.30 below we show that in the class of Besov spaces $\mathbb{B}_{p}^{\Phi}$ the function $\Phi_{r}$ defined in (25) with $r=4$ is the correct one. Interpreting $\Phi_{2}$ as smoothness 1 , the function $\Phi_{4}$ corresponds to the smoothness $1 / 2$. Our approach is similar to [1: First we investigate the functional $N_{T}^{L}$ and then the local time itself by Tanaka's formula.

TheOREM 4.28. Let $d=1, \alpha \in \mathbb{R}$, and

$$
N_{T}^{\alpha}:=\int_{(0, T]} \chi_{\left\{W_{t}>\alpha\right\}} d W_{t} .
$$

Then, for all $p \in(1, \infty)$, one has that

$$
N_{T}^{\alpha} \in \mathbb{B}_{p}^{\Phi_{4}} \backslash\left[\bigcup_{r \in[2,4)} \mathbb{B}_{p}^{\Phi_{r}}\right] .
$$

REMARK 4.29. (1) The natural range for the parameter $r$ in $\Phi_{r}$ is $r \in[2, \infty)$ so that we used the condition $r \in[2,4)$ instead of the equivalent one $r \in(0,4)$.

(2) It follows that $N_{T}^{\alpha} \in \mathbb{B}_{p}^{\Phi_{4}}$ for all $p \in(0, \infty)$, but for the part $N_{T}^{\alpha} \notin$ $\bigcup_{r \in[2,4)} \mathbb{B}_{p}^{\Phi_{r}}$ our argument uses $p>1$.

Proof of Theorem 4.28. (a) Denote $\xi=N_{T}^{\alpha}$. For the part $N_{T}^{\alpha} \in \mathbb{B}_{p}^{\Phi_{4}}$ we only need to consider the case $p \in[2, \infty)$ and let $0 \leq a<b \leq T$. Then, a.s.,

$$
\begin{aligned}
\xi-\xi^{(a, b]}= & \int_{(a, b]} \chi_{\left\{W_{t}>\alpha\right\}} d W_{t}-\int_{(a, b]} \chi_{\left\{W_{t}^{(a, b]}>\alpha\right\}} d W_{t}^{(a, b]} \\
& +\int_{(b, T]}\left[\chi_{\left\{W_{t}>\alpha\right\}}-\chi_{\left\{W_{t}^{(a, b]}>\alpha\right\}}\right] d W_{t}
\end{aligned}
$$

where we use that $\left(\int_{(0, T]} \chi_{\left\{W_{t}>\alpha\right\}} d W_{t}\right)^{(a, b]}=\int_{(0, T]} \chi_{\left\{W_{t}^{(a, b]}>\alpha\right\}} d W_{t}^{(a, b]}$ a.s. which can be proved by approximating the stochastic integral by Riemann sums that converge in $L_{2}$ towards the original integral and to apply the $\cdot(a, b]$-operation to the Riemann sums. Then, by the Burkholder-Davis-Gundy inequalities,

$$
\begin{aligned}
& \left\|\xi-\xi^{(a, b]}\right\|_{p} \\
\leq & 2\left\|\int_{(a, b]} \chi_{\left\{W_{t}>\alpha\right\}} d W_{t}\right\|_{p}+\left\|\int_{(b, T]}\left[\chi_{\left\{W_{t}>\alpha\right\}}-\chi_{\left\{W_{t}^{(a, b]}>\alpha\right\}}\right] d W_{t}\right\|_{p} \\
\leq & \beta_{p}\left[2 \sqrt{b-a}+\left\|\left(\int_{b}^{T}\left|\chi_{\left\{W_{t}>\alpha\right\}}-\chi_{\left\{W_{t}^{(a, b]}>\alpha\right\}}\right|^{2} d t\right)^{\frac{1}{2}}\right\|_{p}\right]
\end{aligned}
$$




$$
=\beta_{p}\left[2 \sqrt{b-a}+\left\|\int_{b}^{T} \chi_{I_{\alpha}\left(W_{b}, W_{b}^{(a, b]}\right)}\left(W_{t}-W_{b}\right) d t\right\|_{q}^{\frac{1}{2}}\right]
$$

for $q:=p / 2 \in[1, \infty)$ and

$$
I_{\alpha}(u, v):=(\alpha-u, \alpha-v] \cup(\alpha-v, \alpha-u]=(\alpha-\max \{u, v\}, \alpha-\min \{u, v\}] .
$$

Let $-\infty<A<B<\infty$ and define the function $f_{A, B}: \mathbb{R} \rightarrow \mathbb{R}$ by

$$
f_{A, B}(x):= \begin{cases}0 & : x \leq A \\ (x-A)^{2} & : A<x<B . \\ (B-A)^{2}+2(B-A)(x-B) & : B \leq x\end{cases}
$$

By the Itô-Tanaka formula and the occupation times formula (see $\mathbf{8 0}$, VI.1.5 and VI.1.6]) we get that, a.s.,

$$
f_{A, B}\left(W_{T}-W_{b}\right)=f_{A, B}(0)+\int_{(b, T]} f_{A, B}^{\prime}\left(W_{t}-W_{b}\right) d W_{t}+\int_{b}^{T} \chi_{(A, B]}\left(W_{t}-W_{b}\right) d t .
$$

This gives that

$$
\begin{aligned}
& \left\|\int_{b}^{T} \chi_{(A, B]}\left(W_{t}-W_{b}\right) d t\right\|_{q} \\
\leq & \left\|f_{A, B}\left(W_{T}-W_{b}\right)-f_{A, B}(0)\right\|_{q}+\left\|\int_{(b, T]} f_{A, B}^{\prime}\left(W_{t}-W_{b}\right) d W_{t}\right\|_{q} \\
\leq & \left\|f_{A, B}^{\prime}\right\|_{\infty}\left[\left\|W_{T}-W_{b}\right\|_{q}+\beta_{q} \sqrt{T-b}\right] \\
= & 2(B-A)\left[\left\|W_{T}-W_{b}\right\|_{q}+\beta_{q} \sqrt{T-b}\right] \\
\leq & 4 \beta_{q} \sqrt{T-b}(B-A) .
\end{aligned}
$$

Then

$$
\begin{aligned}
\left\|\int_{b}^{T} \chi_{I_{\alpha}\left(W_{b}, W_{b}^{(a, b]}\right)}\left(W_{t}-W_{b}\right) d t\right\|_{q} & \leq 4 \beta_{q} \sqrt{T-b}\left\|W_{b}-W_{b}^{(a, b]}\right\|_{q} \\
& \leq 8 \beta_{q} \sqrt{T-b}\left\|W_{b}-W_{a}\right\|_{q} \\
& \leq 8 \beta_{q}^{2} \sqrt{T-b} \sqrt{b-a} .
\end{aligned}
$$

Summarizing gives

$$
\left\|\xi-\xi^{(a, b]}\right\|_{p} \leq \beta_{p}\left[2 \sqrt{b-a}+\left(8 \beta_{q}^{2} \sqrt{T-b} \sqrt{b-a}\right)^{\frac{1}{2}}\right] .
$$

(b) Let us turn to the lower bound, where we assume $p \in(1, \infty)$. We obtain

$$
\begin{aligned}
& \left\|\xi-\xi^{(a, b]}\right\|_{p} \\
\geq & -2\left\|\int_{(a, b]} \chi_{\left\{W_{t}>\alpha\right\}} d W_{t}\right\|_{p}+\left\|\int_{(b, T]}\left[\chi_{\left\{W_{t}>\alpha\right\}}-\chi_{\left\{W_{t}^{(a, b]}>\alpha\right\}}\right] d W_{t}\right\|_{p} \\
\geq & -2 \beta_{p} \sqrt{b-a}+\frac{1}{\beta_{p}}\left\|\int_{b}^{T} \chi_{I_{\alpha}\left(W_{b}, W_{b}^{(a, b]}\right)}\left(W_{t}-W_{b}\right) d t\right\|_{q}^{\frac{1}{2}} .
\end{aligned}
$$


Let $a=0$ and observe that on $\left\{W_{b} \leq-\sqrt{b}, W_{b}^{\prime} \geq \sqrt{b}\right\}$ one has that

$$
I_{\alpha}\left(W_{b}, W_{b}^{(0, b]}\right)=\left(\alpha-W_{b}^{\prime}, \alpha-W_{b}\right] \supseteq(\alpha-\sqrt{b}, \alpha+\sqrt{b}) .
$$

Therefore, for $b \in(0, T / 2)$,

$$
\begin{aligned}
& \left\|\xi-\xi^{(0, b]}\right\|_{p} \\
\geq & -2 \beta_{p} \sqrt{b}+\frac{1}{\beta_{p}} \overline{\mathbb{P}}\left(W_{b} \leq-\sqrt{b}, W_{b}^{\prime} \geq \sqrt{b}\right)^{\frac{1}{2 q}}\left\|\int_{b}^{T} \chi_{(\alpha-\sqrt{b}, \alpha+\sqrt{b})}\left(W_{t}-W_{b}\right) d t\right\|_{q}^{\frac{1}{2}} \\
= & -2 \beta_{p} \sqrt{b}+\frac{1}{\beta_{p}} \overline{\mathbb{P}}\left(W_{1} \leq-1, W_{1}^{\prime} \geq 1\right)^{\frac{1}{2 q}}\left\|\int_{b}^{T} \chi_{(\alpha-\sqrt{b}, \alpha+\sqrt{b})}\left(W_{t}-W_{b}\right) d t\right\|_{q}^{\frac{1}{2}} \\
\geq & -2 \beta_{p} \sqrt{b}+\frac{1}{\beta_{p}} \overline{\mathbb{P}}\left(W_{1} \leq-1, W_{1}^{\prime} \geq 1\right)^{\frac{1}{2 q}}\left\|\int_{b}^{\frac{T}{2}+b} \chi_{(\alpha-\sqrt{b}, \alpha+\sqrt{b})}\left(W_{t}-W_{b}\right) d t\right\|_{q}^{\frac{1}{2}} \\
= & -2 \beta_{p} \sqrt{b}+\frac{1}{\beta_{p}} \overline{\mathbb{P}}\left(W_{1} \leq-1, W_{1}^{\prime} \geq 1\right)^{\frac{1}{2 q}}\left\|\int_{0}^{\frac{T}{2}} \chi_{(\alpha-\sqrt{b}, \alpha+\sqrt{b})}\left(W_{t}\right) d t\right\|_{q}^{\frac{1}{2}} .
\end{aligned}
$$

For the local time of the Brownian motion one has (see [80, Corollary VI.1.9])

$$
L_{t}^{\alpha}=\lim _{\varepsilon \downarrow 0} \frac{1}{2 \varepsilon} \int_{0}^{t} \chi_{(\alpha-\varepsilon, \alpha+\varepsilon)}\left(W_{s}\right) d s \text { a.s. }
$$

Therefore, by Fatou's Lemma,

$$
\liminf _{b \downarrow 0} \frac{1}{\sqrt[4]{b}}\left\|\int_{0}^{\frac{T}{2}} \chi_{(\alpha-\sqrt{b}, \alpha+\sqrt{b})}\left(W_{t}\right) d t\right\|_{q}^{\frac{1}{2}} \geq \sqrt{2\left\|L_{\frac{T}{2}}^{\alpha}\right\|_{q}}>0 .
$$

Because the local time $L_{t}^{\alpha}$ can be expressed by Tanaka's formula by

$$
\frac{1}{2} L_{T}^{\alpha}=\left(W_{T}-\alpha\right)^{+}-\left(W_{0}-\alpha\right)^{+}-N_{T}^{\alpha},
$$

see [80, Theorem VI.1.2], and because $\left(W_{T}-\alpha\right)^{+} \in \mathbb{B}_{p}^{\Phi_{2}}$ for all $p \in(0, \infty)$ we immediately get the following corollary:

Corollary 4.30. For all $\alpha \in \mathbb{R}$ and $p \in(1, \infty)$ one has that

$$
L_{T}^{\alpha} \in \mathbb{B}_{p}^{\Phi_{4}} \backslash\left[\bigcup_{r \in[2,4)} \mathbb{B}_{p}^{\Phi_{r}}\right] .
$$


7 Nov 2017 05:35:26 EST

Prob+Stat

Version 2 - Submitted to Memoirs of the AMS 


\section{CHAPTER 5}

\section{Continuous BMO-Martingales}

The theory of BMO-martingales has become an important tool in the investigation of BSDEs. For an account on this topic the reader is referred, for example, to [32, p. 298] and [22, p. 2922]. In particular, there are two key ingredients that we will use as well: Fefferman's inequality and their generalizations, and the notion of reverse Hölder inequalities. In addition to these two ingredients, we exploit the concept of sliceable BMO-martingales which can be seen as a natural enhancement for the previous techniques. Sliceable BMO-martingales were used by Emery [35, 36] and Schachermayer $\mathbf{8 3}$, and in the context of backward stochastic differential equations by Delbaen and Tang $[\mathbf{3 2}$ and Frei [37.

Throughout this chapter we assume a stochastic basis $\left(A, \mathcal{A}, \mathbb{Q},\left(\mathcal{A}_{t}\right)_{t \in[0, T]}\right), T>0$, where $(A, \mathcal{A}, \mathbb{Q})$ is complete, $\left(\mathcal{A}_{t}\right)_{t \in[0, T]}$ is right-continuous, $\mathcal{A}_{0}$ contains all nullsets, and $\mathcal{A}=\mathcal{A}_{T}$.

\subsection{Continuous BMO-martingales and sliceable numbers}

First we recall the notion of a BMO-martingale.

Definition 5.1. A continuous martingale $M=\left(M_{t}\right)_{t \in[0, T]}$ is of bounded mean oscillation (we write $M \in \mathrm{BMO}$ ) provided that $M_{0} \equiv 0$ and there is constant $c>0$ such that for all stopping times $\tau: A \rightarrow[0, T]$ one has that

$$
\mathbb{E}\left(\left|M_{T}-M_{\tau}\right|^{2} \mid \mathcal{A}_{\tau}\right) \leq c^{2} \text { a.s.. }
$$

We let $\|M\|_{\mathrm{BMO}_{2}}:=\inf c$ where the infimum is taken over all $c>0$ as above.

Next we introduce the sliceable numbers. Without being defined explicitly, these numbers have their origin in an article of Schachermayer 83 and will be used via Theorem 5.25 below in our article. Before giving the definition let us recall the notation

$$
{ }^{\sigma} M^{\tau}:=\left(M_{\tau \wedge t}-M_{\sigma \wedge t}\right)_{t \in[0, T]}
$$

for random times $\sigma, \tau: A \rightarrow[0, T]$ with $0 \leq \sigma \leq \tau \leq T$. 
Definition 5.2. For a (continuous) BMO-martingale $M=\left(M_{t}\right)_{t \in[0, T]}$ and $N \geq 1$ we let

$$
\operatorname{sl}_{N}(M):=\inf \varepsilon
$$

where the infimum is taken over all $\varepsilon>0$ such that there are stopping times $0=\tau_{0} \leq \tau_{1} \leq \cdots \leq \tau_{N}=T$ with

$$
\sup _{k=1, \ldots, N}\left\|^{\tau_{k-1}} M^{\tau_{k}}\right\|_{\mathrm{BMO}_{2}} \leq \varepsilon .
$$

Moreover, we let

$$
\operatorname{sl}_{\infty}(M):=\lim _{N} \operatorname{sl}_{N}(M) .
$$

We call $\operatorname{sl}_{N}(M)$ the $N$-sliceable number of $M$. The (continuous) BMO-martingale $M$ is called sliceable provided that $\operatorname{sl}_{\infty}(M)=0$.

Before we summarise some simple properties of the sliceable numbers we need the following lemma:

Lemma 5.3. Let $0 \leq \sigma \leq \tau \leq T$ be stopping times and $0=\tau_{0} \leq \tau_{1} \leq \cdots \leq \tau_{N}=T$ be a net of stopping times such that for all $\omega \in A$ there is a $k \in\{1, \ldots, N\}$ such that

$$
(\sigma(\omega), \tau(\omega)] \subseteq\left(\tau_{k-1}(\omega), \tau_{k}(\omega)\right] .
$$

Then, for a (continuous) BMO-martingale $N$, one has that

$$
\left\|^{\sigma} N^{\tau}\right\|_{\mathrm{BMO}_{2}} \leq \sup _{k=1, \ldots, N}\left\|^{\tau_{k-1}} N^{\tau_{k}}\right\|_{\mathrm{BMO}_{2}} .
$$

Proof. Let $\rho: A \rightarrow[0, T]$ be a stopping time. Then

$$
\begin{aligned}
\mathbb{E}\left(\left|{ }^{\sigma} N_{T}^{\tau}-{ }^{\sigma} N_{\rho}^{\tau}\right|^{2} \mid \mathcal{A}_{\rho}\right) & =\mathbb{E}\left(\left|N_{\tau \vee \rho}-N_{\sigma \vee \rho}\right|^{2} \mid \mathcal{A}_{\rho}\right) \\
& =\mathbb{E}\left(\mathbb{E}\left(\left|N_{\tau \vee \rho}-N_{\sigma \vee \rho}\right|^{2} \mid \mathcal{A}_{\sigma \vee \rho}\right) \mid \mathcal{A}_{\rho}\right) .
\end{aligned}
$$

Now we observe that $(\bar{\sigma}, \bar{\tau})$ with $\bar{\sigma}:=\sigma \vee \rho$ and $\bar{\tau}:=\tau \vee \rho$ shares the same property as $(\sigma, \tau)$. We let $A_{N+1}:=\{\bar{\sigma}=T\}$, and for $k=1, \ldots, N$,

$$
A_{k}:=\left\{\bar{\sigma} \in\left[\tau_{k-1}, \tau_{k}\right)\right\} .
$$

This gives a partition $A=\bigcup_{k=1}^{N+1} A_{k}$ with $A_{k} \in \mathcal{A}_{\bar{\sigma}}$ and we have that

$$
\begin{aligned}
\mathbb{E}\left(\left|N_{\bar{\tau}}-N_{\bar{\sigma}}\right|^{2} \mid \mathcal{A}_{\bar{\sigma}}\right) & =\sum_{k=1}^{N} \mathbb{E}\left(\chi_{A_{k}}\left|N_{\bar{\tau}}-N_{\bar{\sigma}}\right|^{2} \mid \mathcal{A}_{\bar{\sigma}}\right) \\
& =\sum_{k=1}^{N} \mathbb{E}\left(\chi_{A_{k}}\left|N_{\bar{\tau} \wedge \tau_{k}}-N_{\bar{\sigma} \vee \tau_{k-1}}\right|^{2} \mid \mathcal{A}_{\bar{\sigma}}\right) \\
& =\sum_{k=1}^{N} \mathbb{E}\left(\chi_{A_{k}} \mathbb{E}\left(\left|N_{\bar{\tau} \wedge \tau_{k}}-N_{\bar{\sigma} \vee \tau_{k-1}}\right|^{2} \mid \mathcal{A}_{\bar{\sigma} \vee \tau_{k-1}}\right) \mid \mathcal{A}_{\bar{\sigma}}\right) \\
& \leq \sup _{k=1, \ldots, N}\left\|^{\tau_{k-1}} N^{\tau_{k}}\right\|_{\mathrm{BMO}_{2}}^{2} .
\end{aligned}
$$

To formulate the next result we recall the space $\mathbb{H}_{\infty}$ : 
Definition 5.4. We let $\mathbb{H}_{\infty}$ be the space of all continuous martingales $N=$ $\left(N_{t}\right)_{t \in[0, T]}$ such that $N_{0} \equiv 0$ and

$$
\|N\|_{\mathbb{H}_{\infty}}:=\operatorname{esssup}_{\omega \in A}\langle N\rangle_{T}(\omega)<\infty
$$

where $\left(\langle N\rangle_{t}\right)_{t \in[0, T]}$ denotes the quadratic variation of $\left(N_{t}\right)_{t \in[0, T]}$ (see, for example, 80, Section IV.1]).

It follows directly from the definition that $\mathbb{H}_{\infty} \subseteq \mathrm{BMO}$.

LEMmA 5.5. For (continuous) BMO-martingales $M, M_{1}$, and $M_{2}$ one has the following:

(1) $\operatorname{sl}_{1}(M)=\|M\|_{\mathrm{BMO}_{2}}$.

(2) $\operatorname{sl}_{1}(M) \geq \operatorname{sl}_{2}(M) \geq \cdots \geq 0$.

(3) $\operatorname{sl}_{N_{1}+N_{2}-1}\left(M_{1}+M_{2}\right) \leq \operatorname{sl}_{N_{1}}\left(M_{1}\right)+\operatorname{sl}_{N_{2}}\left(M_{2}\right)$.

(4) $\mathrm{sl}_{\infty}(M)=d_{\mathrm{BMO}_{2}}\left(M, \mathbb{H}_{\infty}\right)$, where

$$
d_{\mathrm{BMO}_{2}}\left(M, \mathbb{H}_{\infty}\right):=\inf \left\{\|M-N\|_{\mathrm{BMO}_{2}}: N \in \mathbb{H}_{\infty}\right\} .
$$

Proof. (1) and (2) are obvious. To prove (3), we assume $\eta>0$ and find nets $0=\tau_{0}^{i} \leq \cdots \leq \tau_{N_{i}}^{i}=T$ such that

$$
\sup _{k=1, \ldots, N_{i}}\left\|\tau_{k-1}^{i} M_{i}^{\tau_{k}^{i}}\right\|_{\mathrm{BMO}_{2}} \leq \operatorname{sl}_{N_{i}}\left(M_{i}\right)+\eta
$$

Now we let $\left(\sigma_{k}\right)_{k=0}^{N_{1}+N_{2}-1}$ be the union of $\left(\tau_{k}^{1}\right)_{k=0}^{N_{1}}$ and $\left(\tau_{k}^{2}\right)_{k=0}^{N_{2}}$ and define the new net $\left(\tau_{k}\right)_{k=0}^{N_{1}+N_{2}-1}$ to be the order statistics of $\left(\sigma_{k}\right)_{k=0}^{N_{1}+N_{2}-1}$, i.e.

$$
\begin{aligned}
& \tau_{0}:=\min _{k} \sigma_{k}=0, \\
& \tau_{N_{1}+N_{2}-1}:=\max _{k} \sigma_{k}=T \text {, } \\
& \tau_{k}:=\quad\left[\begin{array}{c}
I \subseteq\left\{1, \ldots, N_{1}+N_{2}-2\right\} \\
\operatorname{card}(I)=k
\end{array}\right] \max _{l \in I} \sigma_{l} .
\end{aligned}
$$

With this definition and Lemma 5.3 we get for $k=1, \ldots, N_{1}+N_{2}-1$ that

$$
\begin{aligned}
& \left\|^{\tau_{k-1}}\left(M_{1}+M_{2}\right)^{\tau_{k}}\right\|_{\mathrm{BMO}_{2}} \\
\leq & \left\|^{\tau_{k-1}} M_{1}^{\tau_{k}}\right\|_{\mathrm{BMO}_{2}}+\left\|^{\tau_{k-1}} M_{2}^{\tau_{k}}\right\|_{\mathrm{BMO}_{2}} \\
\leq & \sup _{k_{1}=1, \ldots, N_{1}}\left\|\tau_{k_{1}-1}^{1} M_{1}^{\tau_{k_{1}}^{1}}\right\|_{\mathrm{BMO}_{2}}+\sup _{k_{2}=1, \ldots, N_{2}}\left\|\tau_{k_{2}-1}^{2} M_{2}^{\tau_{k_{2}}^{2}}\right\|_{\mathrm{BMO}_{2}} \\
\leq & \operatorname{sl}_{N_{1}}\left(M_{1}\right)+\operatorname{sl}_{N_{2}}\left(M_{2}\right)+2 \eta .
\end{aligned}
$$

By $\eta \downarrow 0$ the assertion follows.

(4) This part is exactly [83. Theorem 1.1, Corollary 1.2], where we have to observe that our setting of a bounded time interval $[0, T]$ does not make a difference compared to $[0, \infty)$ from 83 .

The next example will be used later:

EXAmple 5.6. For a continuous martingale $M=\left(M_{t}\right)_{t \in[0, T]}$ assume that

$$
\langle M\rangle_{t}=\int_{0}^{t} c_{s}^{2} d s, \quad t \in[0, T], \quad \text { a.s. }
$$


for some predictable process $c=\left(c_{t}\right)_{t \in[0, T]}$ and that there is a $\delta>0$ and some $\kappa \in[0, \infty)$ such that

$$
\left[\mathbb{E}\left(\int_{\tau}^{T}\left|c_{s}\right|^{2+\delta} d s \mid \mathcal{A}_{\tau}\right)\right]^{\frac{1}{2+\delta}} \leq \kappa \text { a.s. }
$$

for all stopping times $\tau: A \rightarrow[0, T]$. Then, for $\alpha:=\frac{1}{2}-\frac{1}{2+\delta}>0$, and $N \geq 1$,

$$
\operatorname{sl}_{N}(M) \leq \kappa\left(\frac{T}{N}\right)^{\alpha}
$$

Proof. For $0 \leq a<b \leq T$ we simply get a.s. that

$$
\begin{aligned}
{\left[\mathbb{E}\left(\int_{\tau}^{T} \chi_{(a, b]}(s)\left|c_{s}\right|^{2} d s \mid \mathcal{A}_{\tau}\right)\right]^{\frac{1}{2}} } & =\left[\mathbb{E}\left(\int_{\tau \vee a}^{\tau \vee b}\left|c_{s}\right|^{2} d s \mid \mathcal{A}_{\tau}\right)\right]^{\frac{1}{2}} \\
& \leq\left[\mathbb{E}\left(\int_{\tau \vee a}^{\tau \vee b}\left|c_{s}\right|^{2+\delta} d s \mid \mathcal{A}_{\tau}\right)\right]^{\frac{1}{2+\delta}}(b-a)^{\alpha} \\
& \leq\left[\mathbb{E}\left(\int_{\tau}^{T}\left|c_{s}\right|^{2+\delta} d s \mid \mathcal{A}_{\tau}\right)\right]^{\frac{1}{2+\delta}}(b-a)^{\alpha} \\
& \leq \kappa(b-a)^{\alpha} .
\end{aligned}
$$

Choosing an equidistant partition of $[0, T]$ consisting of $N$ intervals concludes the proof.

\subsection{Fefferman's inequality and $\mathrm{BMO}\left(S_{2 \theta}\right)$ spaces}

In this section we slightly change the point of view: Instead of considering martingales we think in terms of the quadratic variation which is more convenient in the sequel for us. The BMO-spaces, related to backward stochastic differential equations with generators satisfying condition (B3) of Section 6.1 below, are defined as follows:

Definition 5.7. For $\theta \in(0, \infty)$ and an $\mathbb{R}$-valued progressively measurable process $Z=\left(Z_{t}\right)_{t \in[0, T]}$ with $\mathbb{E} \int_{0}^{T}\left|Z_{s}\right|^{2 \theta} d s<\infty$ we let $Z \in \operatorname{BMO}\left(S_{2 \theta}\right)$ provided that

$$
\|Z\|_{\operatorname{BMO}\left(S_{2 \theta}\right)}:=\sup _{t \in[0, T]}\left\|\mathbb{E}\left(\int_{t}^{T}\left|Z_{s}\right|^{2 \theta} d s \mid \mathcal{A}_{t}\right)\right\|_{\infty}^{\frac{1}{2 \theta}}<\infty .
$$

Before we continue we rephrase Definition 5.2 in terms of $\operatorname{BMO}\left(S_{2}\right)$ for the usage in Theorem 6.3 below:

Definition 5.8. For an $\mathbb{R}$-valued progressively measurable process $c=\left(c_{t}\right)_{t \in[0, T]}$, and $N \geq 1$, we let

$$
\mathrm{sl}_{N}^{S_{2}}(c)=\mathrm{sl}_{N}^{S_{2}, \mathbb{A}}(c):=\inf \varepsilon,
$$

where the infimum is taken over all $\varepsilon>0$ such that there are stopping times $0=\tau_{0} \leq \tau_{1} \leq \cdots \leq \tau_{N}=T$ with

$$
\sup _{k=1, \ldots, N}\left\|\left(\chi_{\left(\tau_{k-1}, \tau_{k}\right]}(t) c_{t}\right)_{t \in[0, T]}\right\|_{\mathrm{BMO}\left(S_{2}\right)} \leq \varepsilon
$$


The notation $S_{2 \theta}$ in Definition 5.7 is chosen to indicate that $\operatorname{BMO}\left(S_{2 \theta}\right)$ deals with a modified square function. For $\theta \in(1, \infty)$ we obtain a condition that is stronger than the classical BMO-condition $\|Z\|_{\mathrm{BMO}\left(S_{2}\right)}$, whereas for $\theta \in(0,1)$ the condition gets weaker. If we define

$$
Y_{t}:=\int_{0}^{t}\left|Z_{s}\right|^{2 \theta} d s
$$

then $Z \in \operatorname{BMO}\left(S_{2 \theta}\right)$ if and only if

$$
\sup _{\tau}\left\|\mathbb{E}\left(Y_{T}-Y_{\tau} \mid \mathcal{F}_{\tau}\right)\right\|_{\infty}<\infty
$$

with the supremum taken over all stopping times $\tau: A \rightarrow[0, T]$. This opens the path to apply known results about BMO-spaces to the $\operatorname{BMO}\left(S_{2 \theta}\right)$-spaces. Therefore, by the John-Nirenberg Theorem we get that $Z \in \operatorname{BMO}\left(S_{2 \theta}\right)$ implies that

$$
\int_{0}^{T}\left|Z_{s}\right|^{2 \theta} d s \in L_{\exp }
$$

where the Orlicz space $L_{\exp }$ is given by

$$
\|F\|_{L_{\exp }}:=\inf \left\{\lambda>0: \mathbb{E} e^{\frac{|F|}{\lambda}} \leq 2\right\}
$$

for a random variable $F$ taking values in $\mathbb{R}$, see $[\mathbf{8 4},[38,59$, and [44, Corollary 1].

For the next example the notion of a Banach function space is convenient:

Definition 5.9. A map $\rho: \mathcal{L}_{0}^{+}(A, \mathcal{A}, \mathbb{Q}) \rightarrow[0, \infty]$ defined on the non-negative random variables of $\mathcal{L}_{0}(A, \mathcal{A}, \mathbb{Q})$ is a Banach function norm provided that the following conditions are satisfied:

(1) $\rho(X)=0$ if and only if $X=0$ a.s.

(2) $\rho(X+Y) \leq \rho(X)+\rho(Y)$.

(3) $\rho(\alpha X)=\alpha \rho(X)$ for $\alpha \geq 0$.

(4) $0 \leq X \leq Y$ a.s. implies $\rho(X) \leq \rho(Y)$.

(5) $0 \leq X_{n} \uparrow X$ a.s. implies $\rho\left(X_{n}\right) \uparrow \rho(X)$.

(6) $\rho(1)<\infty$.

(7) There is a $c>0$ such that $\|X\|_{1} \leq c \rho(X)$ for all $X \in \mathcal{L}_{0}^{+}(A, \mathcal{A}, \mathbb{Q})$.

The function $\rho$ is extended to $\|\cdot\|_{E_{\rho}}: L_{0}(A, \mathcal{A}, \mathbb{Q}) \rightarrow[0, \infty]$ by $\|X\|_{E_{\rho}}:=\rho(|X|)$ and we let

$$
E_{\rho}:=\left\{X \in L_{0}(A, \mathcal{A}, \mathbb{Q}):\|X\|_{E_{\rho}}<\infty\right\} .
$$

The spaces $\left[E_{\rho},\|\cdot\|_{E_{\rho}}\right]$ are Banach spaces having the Fatou property, see [10, Theorem 1.1.7].

EXAmple 5.10. Let $T=1$ and assume that $\rho: L_{0}(A, \mathcal{A}, \mathbb{Q}) \rightarrow[0, \infty]$ is a Banach function norm such that for all $t \in(0,1]$ one has that

$$
\sup \left\{\|X\|_{\infty}:\|X\|_{E_{\rho}} \leq 1, X \in L_{0}\left(A, \mathcal{A}_{t}, \mathbb{Q}\right)\right\}=\infty .
$$

Then for all $0<\theta<\eta \leq 1$ there is a progressively measurable process $Z=$ $\left(Z_{t}\right)_{t \in[0, T]}$ such that

(1) $\int_{0}^{T}\left|Z_{t}\right|^{2 \eta} d t \in E_{\rho}$

(2) $Z \in \operatorname{BMO}\left(S_{2 \theta}\right) \backslash \operatorname{BMO}\left(S_{2 \eta}\right)$. 
ProOF. Let $t_{n}:=1-\frac{1}{2^{n}}$ for $n \geq 0$, take

$$
0<\varepsilon<\frac{1}{2 \theta}-\frac{1}{2 \eta},
$$

and choose, for $n \geq 1$ random variables $v_{n}: A \rightarrow \mathbb{R}$ that are $\mathcal{A}_{t_{n}}$-measurable and satisfy

$$
\left\|v_{n}\right\|_{\infty}=2^{(n+1)\left[\frac{1}{2 \eta}+\varepsilon\right]} \quad \text { but } \quad\left\|\left|v_{n}\right|^{2 \eta}\right\|_{E_{\rho}} \leq 1 .
$$

Define the stochastic process $Z=\left(Z_{t}\right)_{t \in[0,1]}$ by

$$
Z_{t}:=\sum_{n=2}^{\infty} \chi_{\left(t_{n-1}, t_{n}\right]}(t) v_{n-1}
$$

Then we get the following three estimates:

(1) For $n \geq 2$ we have

$$
\|Z\|_{\mathrm{BMO}\left(S_{2 \eta}\right)} \geq\left\|v_{n-1}\right\|_{\infty}\left(t_{n}-t_{n-1}\right)^{\frac{1}{2 \eta}}=2^{n\left[\frac{1}{2 \eta}+\varepsilon\right]} 2^{-\frac{n}{2 \eta}} \rightarrow \infty
$$

as $n \rightarrow \infty$, so that $\|Z\|_{\mathrm{BMO}\left(S_{2 \eta}\right)}=\infty$.

(2) We have that

$$
\begin{aligned}
\|Z\|_{\mathrm{BMO}\left(S_{2 \theta}\right)}^{2 \theta} & \leq \sum_{n=2}^{\infty}\left\|v_{n-1}\right\|_{\infty}^{2 \theta}\left(t_{n}-t_{n-1}\right) \\
& =\sum_{n=2}^{\infty} 2^{n\left[\frac{\theta}{\eta}+2 \varepsilon \theta-1\right]}<\infty .
\end{aligned}
$$

(3) On the other side, we have that

$$
\begin{aligned}
\left\|\int_{0}^{T}\left|Z_{t}\right|^{2 \eta} d t\right\|_{E_{\rho}} & \leq \sum_{n=2}^{\infty}\left\|\left|v_{n-1}\right|^{2 \eta}\right\|_{E_{\rho}}\left(t_{n}-t_{n-1}\right) \\
& \leq \sum_{n=2}^{\infty} 2^{-n}<\infty
\end{aligned}
$$

In the following we give a version of the generalized Fefferman's inequality that can be found in [32, Lemma 1.6], see also [5, Theorem 1.1]. Our contribution in Theorem 5.18 below consists in improving the asymptotic behavior of the constant from $p$ to $\sqrt{p}$ in Corollary 5.19 and that the left-hand side in 27 is stronger than the left-hand side in 28 .

We start with the definition of the $\mathbb{H}_{p}\left(S_{2}\right)$-spaces and continue by some elementary lemmas.

Definition 5.11. For $p \in(0, \infty]$ we define $\mathbb{H}_{p}\left(S_{2}\right)$ to be the space of all progressively measurable $\mathbb{R}$-valued process $Z=\left(Z_{t}\right)_{t \in[0, T]}$ such that

$$
\|Z\|_{\mathbb{H}_{p}\left(S_{2}\right)}:=\left\|\left(\int_{0}^{T}\left|Z_{s}\right|^{2} d s\right)^{\frac{1}{2}}\right\|_{p}<\infty .
$$


Lemma 5.12. Let $\mu$ be a finite measure on $\mathcal{B}([0, T])$ with $\mu([0, T])>0, \theta \in(0,1)$, and let

$$
t_{0}:=\inf \{t \in[0, T]: \mu([0, t])>0\}
$$

Then one has that

$$
\int_{\left[t_{0}, T\right]} \mu([0, t])^{\theta-1} d \mu(t) \leq \frac{1}{\theta} \mu([0, T])^{\theta} .
$$

The proof is standard and we leave it to the reader.

Lemma 5.13. Let $p \in(1, \infty), \nu$ be a finite measure on $\mathcal{B}([0, T])$, and $f:[0, T] \rightarrow$ $[0, \infty)$ be non-decreasing and right-hand side continuous. Then

$$
\left|\int_{[0, T]} f(s) d \nu(s)\right|^{p} \leq p \int_{[0, T]}\left|\int_{[0, t]} f(s) d \nu(s)\right|^{p-1} f(t) d \nu(t) .
$$

Proof. For $n \geq 1$ take the equi-spaced grid

$$
0=t_{0}^{n}<t_{1}^{n}<\cdots<t_{2^{n}}^{n}=T .
$$

By dominated convergence it is enough to show that

$$
\begin{aligned}
& \left|f(0) \nu(\{0\})+\sum_{i=1}^{2^{n}} f\left(t_{i}^{n}\right) \nu\left(\left(t_{i-1}^{n}, t_{i}^{n}\right]\right)\right|^{p} \\
\leq & p|f(0) \nu(\{0\})|^{p-1} f(0) \nu(\{0\})+ \\
& p \sum_{i=1}^{2^{n}}\left(f(0) \nu(\{0\})+\sum_{j=1}^{i} f\left(t_{j}^{n}\right) \nu\left(\left(t_{j-1}^{n}, t_{j}^{n}\right]\right)\right)^{p-1} f\left(t_{i}^{n}\right) \nu\left(\left(t_{i-1}^{n}, t_{i}^{n}\right]\right) .
\end{aligned}
$$

Setting $a_{0}:=f(0) \nu(\{0\})$ and $a_{i}:=f\left(t_{i}^{n}\right) \nu\left(\left(t_{i-1}^{n}, t_{i}^{n}\right]\right)$ for $i=1, \ldots, 2^{n}$, this reads as

$$
\left|\sum_{i=0}^{2^{n}} a_{i}\right|^{p} \leq p \sum_{i=0}^{2^{n}}\left(\sum_{j=0}^{i} a_{j}\right)^{p-1} a_{i}
$$

which follows by writing the left-hand side as telescoping sum and applying the mean-value theorem from calculus.

REMARK 5.14. In Lemmas 5.12 and 5.13 the factors $1 / \theta$ and $p$ are sharp, but one does not have equalities in general (one can check the cases where $\mu$ and $\nu$ are either the Lebesgue measure or the Dirac measure at (say) $T$, and $f \equiv 1$ ).

Definition 5.15. We call a map

$$
\nu: A \times \mathcal{B}([0, T]) \rightarrow[0, \infty)
$$

adapted random measure provided that

(1) the map $\nu(\omega, \cdot): \mathcal{B}([0, T]) \rightarrow[0, \infty)$ is a measure for all $\omega \in A$,

(2) the map $\nu(\cdot,[0, t]): A \rightarrow \mathbb{R}$ is $\mathcal{A}_{t}$-measurable for all $t \in[0, T]$.

Moreover, we let

$$
\|\nu\|_{\mathrm{BMO}}:=\sup _{t \in[0, T]}\left\|\mathbb{E}\left(\nu([t, T]) \mid \mathcal{A}_{t}\right)\right\|_{\infty} .
$$


Given any non-negative, non-decreasing, left-hand side continuous, and adapted process $(f(s))_{s \in[0, T]}$, the process $\left(\int_{[0, t]} f(s) d \nu(s)\right)_{t \in[0, T]}$ is well defined, non-decreasing, right-hand side continuous, and adapted.

Lemma 5.16. Let $\nu$ be an adapted random measure, $(f(t))_{t \in[0, T]}$ be non-decreasing, adapted, non-negative, and left-hand side continuous. Then, one has that

$$
\mathbb{E} \int_{[0, T]} f(s) d \nu(s) \leq \mathbb{E} f(T)\|\nu\|_{\mathrm{BMO}}
$$

Proof. We can assume that $\mathbb{E} f(T)\|\nu\|_{\text {BMO }}<\infty$, otherwise there is nothing to prove. Assuming the equi-spaced net

$$
0=t_{0}^{n}<\cdots<t_{2^{n}}^{n}=T,
$$

it is sufficient to show that

$$
\mathbb{E} \sum_{i=0}^{2^{n}-1} f\left(t_{i}^{n}\right) \nu\left(\left[t_{i}^{n}, t_{i+1}^{n}\right)\right)+\mathbb{E} f(T) \nu(\{T\}) \leq \mathbb{E} f(T) \sup _{j=0, \ldots, 2^{n}}\left\|\mathbb{E}\left(\nu\left(\left[t_{j}^{n}, T\right]\right) \mid \mathcal{A}_{t_{j}^{n}}\right)\right\|_{\infty} .
$$

Letting $q_{i}^{n}:=\nu\left(\left[t_{i}^{n}, t_{i+1}^{n}\right)\right)$ for $i=0, \ldots, 2^{n}-1, q_{2^{n}}^{n}:=\nu(\{T\})$, and $a_{0}^{n}+\cdots+a_{i}^{n}=$ $f\left(t_{i}^{n}\right)$, we get that

$$
\begin{aligned}
& \mathbb{E}\left[\sum_{i=0}^{2^{n}-1} f\left(t_{i}^{n}\right) \nu\left(\left[t_{i}^{n}, t_{i+1}^{n}\right)\right)+f(T) \nu(\{T\})\right] \\
&=\mathbb{E}\left[\sum_{0 \leq j \leq i \leq 2^{n}} a_{j}^{n} q_{i}^{n}\right] \\
&=\sum_{j=0}^{2^{n}} \mathbb{E}\left[a_{j}^{n} \mathbb{E}\left(q_{j}^{n}+\cdots+q_{2^{n}}^{n} \mid \mathcal{A}_{t_{j}^{n}}\right)\right] \\
& \leq \mathbb{E} f(T) \sup _{j=0, \ldots, 2^{n}}\left\|\mathbb{E}\left(\nu\left(\left[t_{j}^{n}, T\right]\right) \mid \mathcal{A}_{t_{j}^{n}}\right)\right\|_{\infty} .
\end{aligned}
$$

Lemma 5.17. Let $\mu$ and $\nu$ be adapted random measures such that $(\mu(\cdot,[0, t]))_{t \in[0, T]}$ and $(\nu(\cdot,[0, t]))_{t \in[0, T]}$ are continuous processes. Let $\eta \in(0,1), p \in(1, \infty)$, and assume that

Then we have that

$$
\mathbb{E}\left|\int_{[0, T]} \mu([0, t])^{\eta} d \nu(t)\right|^{p}<\infty .
$$

$$
\left\|\int_{[0, T]} \mu([0, t])^{\eta} d \nu(t)\right\|_{p} \leq p\left\|\mu([0, T])^{\eta}\right\|_{p}\|\nu\|_{\mathrm{BMO}} .
$$

Proof. For $p \in(1, \infty)$ we use Lemma 5.13 and Lemma 5.16 to get that

$$
\begin{aligned}
& \mathbb{E}\left|\int_{[0, T]} \mu([0, t])^{\eta} d \nu(t)\right|^{p} \\
\leq & p \mathbb{E} \int_{[0, T]}\left|\int_{[0, t]} \mu([0, s])^{\eta} d \nu(s)\right|^{p-1} \mu([0, t])^{\eta} d \nu(t)
\end{aligned}
$$




$$
\begin{aligned}
& \leq p \mathbb{E}\left[\left|\int_{[0, T]} \mu([0, s])^{\eta} d \nu(s)\right|^{p-1} \mu([0, T])^{\eta}\right]\|\nu\|_{\mathrm{BMO}} \\
& \leq p\left[\mathbb{E}\left|\int_{[0, T]} \mu([0, t])^{\eta} d \nu(t)\right|^{p}\right]^{\frac{p-1}{p}}\left\|\mu([0, T])^{\eta}\right\|_{p}\|\nu\|_{\mathrm{BMO}} .
\end{aligned}
$$

Dividing by $\left[\mathbb{E}\left|\int_{[0, T]} \mu([0, t])^{\eta} d \nu(t)\right|^{p}\right]^{\frac{p-1}{p}}$ in the case this expression is positive (otherwise there is nothing to prove), gives the desired inequality.

TheOREM 5.18. Let $\mu, \nu$ be adapted random measures such that $(\mu(\cdot,[0, t]))_{t \in[0, T]}$ and $(\nu(\cdot,[0, t]))_{t \in[0, T]}$ are continuous processes and $\mu(\omega,\{0\})>0$ for all $\omega \in A$. Let $p \in(1, \infty)$ and assume that

$$
\mathbb{E}\left|\int_{[0, T]} \mu([0, t])^{\frac{1}{2}} d \nu(t)\right|^{p}<\infty .
$$

Then we have that

$$
\left\|\int_{[0, T]} \mu([0, t])^{-\frac{1}{2}} d \mu(t)\right\|_{p}\left\|\int_{[0, T]} \mu([0, t])^{\frac{1}{2}} d \nu(t)\right\|_{p} \leq 2 p\|\mu([0, T])\|_{\frac{p}{2}}\|\nu\|_{\mathrm{BMO}}
$$

Proof. For $\theta=1 / 2$ Lemma 5.12 gives that

$$
\left\|\int_{[0, T]} \mu([0, t])^{-\frac{1}{2}} d \mu(t)\right\|_{p} \leq 2\left[\mathbb{E} \mu([0, T])^{\frac{p}{2}}\right]^{\frac{1}{p}}=2 \sqrt{\|\mu([0, T])\|_{\frac{p}{2}}} .
$$

Moreover, by Lemma 5.17 applied to $\eta=1 / 2$,

$$
\left\|\int_{[0, T]} \mu([0, t])^{\frac{1}{2}} d \nu(t)\right\|_{p} \leq p\left\|\mu([0, T])^{\frac{1}{2}}\right\|_{p}\|\nu\|_{\mathrm{BMO}}=p \sqrt{\|\mu([0, T])\| \frac{p}{2}}\|\nu\|_{\mathrm{BMO}}
$$

Corollary 5.19. Let $\left(A_{t}\right)_{t \in[0, T]}$ and $\left(B_{t}\right)_{t \in[0, T]}$ be progressively measurable $\mathbb{R}$ valued processes such that $\mathbb{E} \int_{0}^{T}\left|B_{t}\right|^{2} d t<\infty$ and $p \in[1, \infty)$. Then one has that

$$
\left\|\int_{0}^{T}\left|A_{t} B_{t}\right| d t\right\|_{p} \leq c \sqrt{5.19}, p,\|A\|_{\mathbb{H}_{p}\left(S_{2}\right)}\|B\|_{\mathrm{BMO}\left(S_{2}\right)}
$$

with $c_{5.19, p}:=\sqrt{2 p}$. If the optimal constant in 28 is denoted by $c_{5.19, p}^{\mathrm{opt}}$, then

$$
\inf _{p \in[2, \infty)} \frac{c_{[5.19 p, p}^{\text {opt }}}{\sqrt{p}}>0,
$$

i.e. the order of magnitude $\sqrt{p}$ of $c \sqrt[5.19, p]{ }$ as $p \rightarrow \infty$ is optimal.

Proof. (1) We verify the inequality 28). We first assume that there is a $c>0$ such that $\left|A_{s}(\omega)\right| \leq c$ and $\left|B_{s}(\omega)\right| \leq c$ for all $(s, \omega) \in[0, T] \times A$. For $\varepsilon>0$ and the Dirac measure $\delta_{0}$ in 0 define

$$
d \mu_{\varepsilon}(t):=\varepsilon d \delta_{0}(t)+A_{t}^{2} d t \quad \text { and } \quad d \nu(t):=B_{t}^{2} d t .
$$


Then, by Theorem 5.18

$$
\begin{aligned}
& \left\|\int_{0}^{T}\left|A_{t} B_{t}\right| d t\right\|_{p} \\
& \leq\left\|\sqrt{\int_{0}^{T}\left|\varepsilon+\int_{0}^{t} A_{s}^{2} d s\right|^{-\frac{1}{2}}\left|A_{t}\right|^{2} d t}\right\|_{2 p}\left\|\sqrt{\int_{0}^{T}\left|\varepsilon+\int_{0}^{t} A_{s}^{2} d s\right|^{\frac{1}{2}}\left|B_{t}\right|^{2} d t}\right\|_{2 p} \\
& \leq\left\|\int_{[0, T]}\left|\mu_{\varepsilon}([0, t])\right|^{-\frac{1}{2}} d \mu_{\varepsilon}(t)\right\|_{p}^{\frac{1}{2}}\left\|\int_{[0, T]}\left|\mu_{\varepsilon}([0, t])\right|^{\frac{1}{2}} d \nu(t)\right\|_{p}^{\frac{1}{2}} \\
& \leq \sqrt{2 p\left\|\mu_{\varepsilon}([0, T])\right\|_{\frac{p}{2}}\|\nu\|_{\mathrm{BMO}}} \\
& =\sqrt{2 p}\left\|\left(\varepsilon+\int_{0}^{T}\left|A_{t}\right|^{2} d t\right)^{\frac{1}{2}}\right\| \sup _{p}\left\|\mathbb{E}\left(\int_{t}^{T}\left|B_{s}\right|^{2} d s \mid \mathcal{A}_{t}\right)\right\|_{\infty}^{\frac{1}{2}} .
\end{aligned}
$$

By $\varepsilon \downarrow 0$ we get that

$$
\left\|\int_{0}^{T}\left|A_{s} B_{s}\right| d s\right\|_{p} \leq \sqrt{2 p}\left\|\left(\int_{0}^{T}\left|A_{t}\right|^{2} d t\right)^{\frac{1}{2}}\right\| \sup _{p}\left\|\mathbb{E}\left(\int_{t}^{T}\left|B_{s}\right|^{2} d s \mid \mathcal{A}_{t}\right)\right\|_{\infty}^{\frac{1}{2}}
$$

whenever $\left|A_{s}(\omega)\right| \leq c$ and $\left|B_{s}(\omega)\right| \leq c$ for all $(s, \omega) \in[0, T] \times A$. By monotone convergence we can omit the restriction on $A$ first, and finally we can do so for $B$ as well.

(2) We verify the inequality 29 and assume w.lo.g. that $T=1$ (otherwise we apply a re-scaling). Let $A=B \in \operatorname{BMO}\left(S_{2}\right)$ and set $C_{t}:=A_{t}^{2}$. Then

$$
\left\|\int_{0}^{T} C_{t} d t\right\| \leq c_{p}^{\mathrm{opt}}\left[\begin{array}{l}
5.19, p \\
\text {, }
\end{array} \int_{0}^{T} C_{t} d t\left\|_{\frac{p}{2}}^{\frac{1}{2}} \sup _{t \in[0, T]}\right\| \mathbb{E}\left(\int_{t}^{T} C_{s} d s \mid \mathcal{A}_{t}\right) \|_{\infty}^{\frac{1}{2}} .\right.
$$

Assume that we can choose $A=B \in \operatorname{BMO}\left(S_{2}\right)$ with

(1) $M:=\sup _{t \in[0, T]}\left\|\mathbb{E}\left(\int_{t}^{T} C_{s} d s \mid \mathcal{A}_{t}\right)\right\|_{\infty}<\infty$

(2) and such that there exists a $c \in[1, \infty)$ such that for all $p \in[1, \infty)$ one has

$$
\frac{p}{c} \leq\left\|\int_{0}^{T} C_{t} d t\right\|_{p} \leq c p .
$$

Then we would get that $\frac{p}{c} \leq c_{5.19, p}^{\text {opt }} \sqrt{c_{\frac{p}{2}}} \sqrt{M}$ for $p \in[2, \infty)$ and therefore

$$
c_{5.19, p}^{\mathrm{opt}} \geq \frac{\sqrt{p}}{c \sqrt{c M}} \sqrt{2} \text {. }
$$

Now we construct the process $C$. The probability space $(A, \mathcal{A}, \mathbb{Q})$ we define by $A:=\{1,2,3, \ldots\}$ and $\mathbb{Q}(\{k\}):=2^{-k}$ for $k \geq 1$, where $\mathcal{A}$ is the system of all subsets of $A$. The right continuous filtration is constructed in two steps. First we set $\mathcal{A}_{t_{0}}:=\{\emptyset, A\}$ and $\mathcal{A}_{t_{l}}:=\sigma(\{1\}, \ldots,\{l\})$ for $l \geq 1$, where $t_{l}:=1-2^{-l}$ for 
$l=0,1,2, \ldots$ Then this is extended to $\left(\mathcal{A}_{t}\right)_{t \in[0,1]}$ by $\mathcal{A}_{t}:=\mathcal{A}_{t_{l}}$ if $t \in\left[t_{l}, t_{l+1}\right)$. Finally we define the progressively measurable process $\left(C_{t}\right)_{t \in[0,1]}$ by

$$
C_{t}:=\sum_{l=1}^{\infty} 2^{l} 1_{\left(t_{l-1}, t_{l}\right]}(t) 1_{\{l, l+1, \ldots\}} \quad \text { for } \quad t \in[0,1] .
$$

For $\xi:=\int_{0}^{1} C_{t} d t$ one gets $\mathbb{Q}(|\xi|=k)=\frac{1}{2^{k}}$ for $k=1,2, \ldots$ so that (by a standard computation using the Gamma function and Stirling's formula) one has the twosided estimate $\frac{p}{c} \leq\|\xi\|_{p} \leq c p$ for all $p \in[1, \infty)$ and some $c \in[1, \infty)$. On the other hand,

$$
\int_{\{l, l+1, \ldots\}}\left[\int_{t_{l-1}}^{1} C_{s} d s\right] d \mathbb{Q} \leq 2 \mathbb{Q}(\{l, l+1, \ldots\}) \quad \text { for } \quad l \geq 1
$$

which implies that $\sup _{t \in[0, T]}\left\|\mathbb{E}\left(\int_{t}^{T} C_{s} d s \mid \mathcal{A}_{t}\right)\right\|_{\infty} \leq 2$.

REMARK 5.20. There is a connection to the Bhattacharyya coefficient (also called Hellinger coefficient) of two measures, see [12. Assume two Borel measures $\mu, \nu$ on $\mathcal{B}([0, T])$ and a reference measure $\sigma$ such that $\mu$ and $\nu$ are absolutely continuous with respect to $\sigma$. Then

$$
B(\mu, \nu):=\int_{[0, T]} \sqrt{\frac{d \mu}{d \sigma} \frac{d \nu}{d \sigma}} d \sigma,
$$

which is independent from the particular choice of the reference measure, is called Bhattacharyya coefficient. Under the assumptions of Corollary 5.19 with $d \mu(t):=$ $A_{t}^{2} d t$ and $d \nu(t)=B_{t}^{2} d t$, we have

$$
B(\mu(\omega, \cdot), \nu(\omega, \cdot))=\int_{0}^{T}\left|A_{s}(\omega) B_{s}(\omega)\right| d s .
$$

Corollary 5.21. For $\theta \in(0,1], p \in[1, \infty)$, and $Z \in \mathbb{H}_{p}\left(S_{2}\right) \cap \operatorname{BMO}\left(S_{2 \theta}\right)$ one has

$$
\left.\left.\mathbb{E}\left|\int_{0}^{T}\right| Z_{t}\right|^{1+\theta} d t\right|^{p}<\infty
$$

with

$$
\left\|\int_{0}^{T}\left|Z_{t}\right|^{1+\theta} d t\right\|_{p} \leq c \sqrt{5.19,, p}\|Z\|_{\mathbb{H}_{p}\left(S_{2}\right)}\|Z\|_{\mathrm{BMO}\left(S_{2 \theta}\right)}^{\theta}
$$

Remark 5.22. (1) For $\theta=1$ we have that $\operatorname{BMO}\left(S_{2 \theta}\right) \subseteq \mathbb{H}_{p}\left(S_{2}\right)$ because of relation (26).

(2) In general, for $\theta \in(0,1)$ we do not have $\operatorname{BMO}\left(S_{2 \theta}\right) \subseteq \mathbb{H}_{p}\left(S_{2}\right)$ (here one can take deterministic processes) nor $\mathbb{H}_{p}\left(S_{2}\right) \subseteq \operatorname{BMO}\left(S_{2 \theta}\right)$ (see Example 5.10 .

(3) In general, neither the condition $Z \in \mathbb{H}_{p}\left(S_{2}\right)$ implies $\left.\left.\mathbb{E}\left|\int_{0}^{T}\right| Z_{s}\right|^{1+\theta} d s\right|^{p}<$ $\infty$ for $\theta \in(0,1]$, nor $Z \in \operatorname{BMO}\left(S_{2 \theta}\right)$ does for $\theta \in(0,1)$. 


\subsection{Reverse Hölder inequalities}

So far, we assumed a stochastic basis $\left(A, \mathcal{A}, \mathbb{Q},\left(\mathcal{A}_{t}\right)_{t \in[0, T]}\right), T>0$, where $(A, \mathcal{A}, \mathbb{Q})$ is complete, $\left(\mathcal{A}_{t}\right)_{t \in[0, T]}$ is right-continuous, $\mathcal{A}_{0}$ contains all null-sets, and $\mathcal{A}=$ $\mathcal{A}_{T}$. To be in accordance with $\mathbf{5 9}$, we additionally assume now that all local martingales are continuous. As we work on a closed time-interval we have to explain our understanding of a local martingale: we require that the localizing sequence of stopping times $0 \leq \tau_{1} \leq \tau_{2} \leq \cdots \leq T$ satisfies $\lim _{n} \mathbb{P}\left(\tau_{n}=T\right)=1$. So we extend the filtration by $\mathcal{A}_{T}$ to $(T, \infty)$, i.e. $\mathcal{A}_{t}:=\mathcal{A}_{T}$ for $t \in(T, \infty)$, and extend all local martingales $\left(N_{t}\right)_{t \in[0, T]}$ (in our setting) by $N_{T}$ to $(T, \infty)$. This yields the standard notion of a local martingale.

The probabilistic Muckenhoupt weights provide a natural way to verify various martingale inequalities after a change of measure, see exemplary [57, 15, [59. This change of measure will appear in our setting in terms of a Girsanov transformation that removes a sub-quadratic or quadratic drift term in $Z$ that originates from the generator of our BSDE, see Section 5.4 .

Definition 5.23. Assume a martingale $M=\left(M_{t}\right)_{t \in[0, T]}$ with $M_{0} \equiv 0$ such that $\mathcal{E}(M)$ with

$$
\mathcal{E}(M)_{t}=e^{M_{t}-\frac{1}{2}\langle M\rangle_{t}}
$$

for $t \in[0, T]$ is a martingale as well. For $\beta \in(1, \infty)$ we let $\mathcal{E}(M) \in \mathcal{R H}_{\beta}$ provided that there is a constant $c>0$ such that for all stopping times $\tau: A \rightarrow[0, T]$ one has that

$$
\mathbb{E}\left(\left|\mathcal{E}(M)_{T}\right|^{\beta} \mid \mathcal{A}_{\tau}\right)^{\frac{1}{\beta}} \leq c \mathcal{E}(M)_{\tau} \text { a.s. }
$$

The smallest possible $c \geq 0$ is denoted by $\mathcal{R H}_{\beta}(\mathcal{E}(M))$.

It is known [59, Theorem 2.3] that $\mathcal{E}(M)$ is a martingale for $M \in$ BMO. Moreover, we have the following result:

Proposition 5.24 (599, Theorems 2.4 and 3.4]). Let $M$ be a martingale with $M_{0} \equiv 0$ such that $\mathcal{E}(M)$ is a martingale. Then $M \in \mathrm{BMO}$ if and only if $\mathcal{E}(M) \in$ $\bigcup_{\beta \in(1, \infty)} \mathcal{R H}_{\beta}$.

Later in our application we need to know whether a certain martingale $M$ generates a Doléan-Dade exponential that satisfies a reverse Hölder inequality. Here the $\mathrm{BMO}_{2}$-distance to $L_{\infty}$ would be a natural candidate for the extreme case that the reverse Hölder inequality is satisfied for all parameters $\beta \in(1, \infty)$, as Kazamaki 59. Theorem 3.8] provides the characterization $M \in{\overline{L_{\infty}}}^{\left[\mathrm{BMO},\|\cdot\|_{\mathrm{BMO}_{2}}\right]}$ for this case. On the other hand, Grandits [49] has shown that a positive $\mathrm{BMO}_{2}$-distance to $L_{\infty}$ does not provide a reasonable estimate for the critical value of $\beta$ such that one has a reverse Hölder inequality (see also the Note added in Proof of [83]). This is our reason to use the concept sliceable (which describes the $\mathrm{BMO}_{2}$-distance to $\mathbb{H}_{\infty}$ due to the result of Schachermayer [83]) because the following observation yields explicit estimates for the critical exponent $\beta$ and the corresponding multiplicative constants in the reverse Hölder inequalities: 
Theorem 5.25. Let $\Phi:(1, \infty) \rightarrow(0, \infty)$ be a non-increasing function and let

$$
\Psi:\{(\gamma, \beta) \in[0, \infty) \times(1, \infty): 0 \leq \gamma<\Phi(\beta)<\infty\} \rightarrow[0, \infty)
$$

be right-continuous in its first argument and such that

$$
\Psi\left(\gamma_{1}, \beta\right) \leq \Psi\left(\gamma_{2}, \beta\right) \quad \text { for } \quad 0 \leq \gamma_{1} \leq \gamma_{2}<\Phi(\beta),
$$

with the property that

$$
\|M\|_{\mathrm{BMO}_{2}}<\Phi(\beta) \quad \text { implies } \quad \mathcal{R H}_{\beta}(\mathcal{E}(M)) \leq \Psi\left(\|M\|_{\mathrm{BMO}_{2}}, \beta\right) .
$$

Then, for $\operatorname{sl}_{N}(M)<\Phi(\beta)$ we have that $\mathcal{R} \mathcal{H}_{\beta}(\mathcal{E}(M)) \leq\left[\Psi\left(\mathrm{sl}_{N}(M), \beta\right)\right]^{N}$.

Proof. The proof is based on a simple recursion argument that uses the concept of a sliceable BMO-martingale. For $\mathrm{sl}_{N}(M)<\Phi(\beta)$ we choose $0=\tau_{0} \leq \cdots \leq$ $\tau_{N}=T$ such that

$$
\left\|^{\tau_{k-1}} M^{\tau_{k}}\right\|_{\mathrm{BMO}_{2}}<\mathrm{sl}_{N}(M)+\eta<\Phi(\beta)
$$

for some $\eta>0$ and all $k=1, \ldots, N$. Therefore,

$$
\mathcal{R H}_{\beta}\left(\mathcal{E}\left({ }^{\tau_{k-1}} M^{\tau_{k}}\right)\right) \leq \Psi\left(\left\|{ }^{\tau_{k-1}} M^{\tau_{k}}\right\|_{\mathrm{BMO}_{2}}, \beta\right) \leq \Psi\left(\mathrm{sl}_{N}(M)+\eta, \beta\right) .
$$

Letting $\tau: A \rightarrow[0, T]$ be a stopping time and $\sigma_{k}:=\tau_{k} \vee \tau$ gives that

$$
\begin{aligned}
& \mathbb{E}_{\mathcal{A}_{\tau}}\left(e^{\beta\left(M_{T}-\frac{1}{2}\langle M\rangle_{T}\right)}\right) \\
& =\left(e^{\beta\left(M_{\tau}-\frac{1}{2}\langle M\rangle_{\tau}\right)}\right) \mathbb{E}_{\mathcal{A}_{\tau}}\left(e^{\beta\left(\left[M_{T}-M_{\tau}\right]-\frac{1}{2}\left[\langle M\rangle_{T}-\langle M\rangle_{\tau}\right]\right)}\right) \\
& =\left(e^{\beta\left(M_{\tau}-\frac{1}{2}\langle M\rangle_{\tau}\right)}\right) \mathbb{E}_{\mathcal{A}_{\tau}}\left(\prod_{k=1}^{N} e^{\beta\left(\left[M_{\sigma_{k}}-M_{\sigma_{k-1}}\right]-\frac{1}{2}\left[\langle M\rangle_{\sigma_{k}}-\langle M\rangle_{\sigma_{k-1}}\right]\right)}\right) .
\end{aligned}
$$

Next we observe that

$$
\mathbb{E}_{\mathcal{A}_{\sigma_{k-1}}}\left(e^{\beta\left(\left[M_{\sigma_{k}}-M_{\sigma_{k-1}}\right]-\frac{1}{2}\left[\langle M\rangle_{\sigma_{k}}-\langle M\rangle_{\sigma_{k-1}}\right]\right)}\right) \leq\left[\Psi\left(\operatorname{sl}_{N}(M)+\eta, \beta\right)\right]^{\beta}
$$

for $k=1, \ldots, N$ which follows from

$$
\left\|^{\sigma_{k-1}} M^{\sigma_{k}}\right\|_{\mathrm{BMO}_{2}}=\left\|^{\tau \vee \tau_{k-1}} M^{\tau \vee \tau_{k}}\right\|_{\mathrm{BMO}_{2}} \leq \sup _{l=1, \ldots, N}\left\|^{\tau_{l-1}} M^{\tau_{l}}\right\|_{\mathrm{BMO}_{2}}<\operatorname{sl}_{N}(M)+\eta,
$$

where we use Lemma 5.3. Applying (30) inductively backwards beginning with $k=N$ and using the projection property of the conditional expectation gives that

$$
\mathcal{R H}_{\beta}(\mathcal{E}(M))^{\beta} \leq\left[\Psi\left(\mathrm{sl}_{N}(M)+\eta, \beta\right)\right]^{\beta N} .
$$

We conclude by $\eta \downarrow 0$.

According to [59, Proof of Theorem 3.1] possible choices of $(\Phi, \Psi)$ are

$$
\begin{aligned}
\Phi(\beta) & :=\left(1+\frac{1}{\beta^{2}} \log \left(1+\frac{1}{2 \beta-2}\right)\right)^{\frac{1}{2}}-1, \\
\Psi(\gamma, \beta) & :=\left(\frac{2}{1-\frac{2 \beta-2}{2 \beta-1} e^{\beta^{2}\left[\gamma^{2}+2 \gamma\right]}}\right)^{\frac{1}{\beta}},
\end{aligned}
$$

where $\Phi$ is decreasing with $\lim _{\beta \rightarrow \infty} \Phi(\beta)=0$ and $\lim _{\beta \rightarrow 1} \Phi(\beta)=\infty$. 


\subsection{An application to BSDEs}

In this section we follow the ideas of [22, Proof of Proposition 2.3] but adapt and extend the ideas for our purpose. Let $B=\left(B_{t}\right)_{t \in[0, T]}$ be an $n$-dimensional standard Brownian motion (where all paths are continuous) on a basis $\left(A, \mathcal{A}, \mathbb{Q},\left(\mathcal{A}_{t}\right)_{t \in[0, T]}\right)$, where $(A, \mathcal{A}, \mathbb{Q})$ is complete, $\left(\mathcal{A}_{t}\right)_{t \in[0, T]}$ is the augmentation of the natural filtration of $B$, and $\mathcal{A}_{T}=\mathcal{A}$. It is known (see [77, Section IV.3]) that the conditions of Section 5.3 are satisfied. We consider the two backward equations

$$
\begin{aligned}
Y_{t}^{0} & =\xi^{0}+\int_{t}^{T} f^{0}\left(s, Y_{s}^{0}, Z_{s}^{0}\right) d s-\int_{t}^{T} Z_{s}^{0} d B_{s}, \\
Y_{t}^{1} & =\xi^{1}+\int_{t}^{T} f^{1}(s) d s-\int_{t}^{T} Z_{s}^{1} d B_{s}
\end{aligned}
$$

where we assume the following conditions:

(D1) The processes $f^{1}, Z^{0}$ and $Z^{1}$ are predictable and the processes $Y^{0}$ and $Y^{1}$ continuous and adapted,

(D2) $\mathbb{E}\left|\xi^{i}\right|^{2}<\infty$ and $\mathbb{E} \int_{0}^{T}\left|Z_{s}^{i}\right|^{2} d s<\infty$ for $i=0,1$,

(D3) $\mathbb{E}\left|\int_{0}^{T}\right| f^{0}\left(s, Y_{s}^{0}, Z_{s}^{0}\right)|d s|^{2}<\infty$ and $\mathbb{E}\left|\int_{0}^{T}\right| f^{1}(s)|d s|^{2}<\infty$,

(D4) the generator $f^{0}: \Omega_{T} \times \mathbb{R} \times \mathbb{R}^{n} \rightarrow \mathbb{R}$ is such that $(t, \omega) \mapsto f^{0}(t, \omega, y, z)$ is predictable for all $(y, z),(y, z) \rightarrow f^{0}(t, \omega, y, z)$ is continuous for all $(t, \omega)$, and there is an $L_{Y} \geq 0$ such that, for all $\left(t, \omega, y_{0}, y_{1}, z\right)$,

$$
\left|f^{0}\left(t, \omega, y_{0}, z\right)-f^{0}\left(t, \omega, y_{1}, z\right)\right| \leq L_{Y}\left|y_{0}-y_{1}\right| .
$$

We let $\Delta \xi:=\xi^{1}-\xi^{0}$, and for $s \in[0, T]$,

$$
\begin{aligned}
\Delta Y_{s} & :=Y_{s}^{1}-Y_{s}^{0}, \\
\Delta Z_{s} & :=Z_{s}^{1}-Z_{s}^{0}, \\
a_{s} & :=f^{1}(s)-f^{0}\left(s, Y_{s}^{1}, Z_{s}^{1}\right), \\
c_{s} & :=\frac{f^{0}\left(s, Y_{s}^{0}, Z_{s}^{1}\right)-f^{0}\left(s, Y_{s}^{0}, Z_{s}^{0}\right)}{\left|\Delta Z_{s}\right|^{2}} \chi_{\left\{\Delta Z_{s} \neq 0\right\}} \Delta Z_{s}, \\
\Xi_{s} & :=|\Delta \xi|+\int_{s}^{T}\left|a_{r}\right| d r .
\end{aligned}
$$

Lemma 5.26. Assume that $c=\left(c_{t}\right)_{t \in[0, T]} \in \operatorname{BMO}\left(S_{2}\right)$ with $\|c \mid\|_{\mathrm{BMO}\left(S_{2}\right)} \leq \gamma<\infty$, $\lambda_{t}:=\exp \left(\int_{0}^{t} c_{s} d B_{s}-\frac{1}{2} \int_{0}^{t}\left|c_{s}\right|^{2} d s\right)$ and $p_{0} \in(1, \infty)$ such that $\mathcal{R} \mathcal{H}_{p_{0}^{\prime}}(\lambda) \leq \rho<\infty$ with $1=\left(1 / p_{0}\right)+\left(1 / p_{0}^{\prime}\right)$. Assume $p \in[2, \infty)$ with $p>p_{0}$ such that

$$
\left.\left.\mathbb{E}\left|\int_{0}^{T}\right| \Delta Z_{s}\right|^{2} d s\right|^{\frac{p}{2}}<\infty
$$

Then there is a c $\sqrt{5.26} \in(0, \infty)$, depending at most on $\left(T, L_{Y}, p, p_{0}, \gamma, \rho, n\right)$, such that for all $t \in[0, T]$ one has that

$$
\left\|\sup _{s \in[t, T]}\left|\Delta Y_{s}\right|\right\|_{p}+\left\|\left(\int_{t}^{T}\left|\Delta Z_{s}\right|^{2} d s\right)^{\frac{1}{2}}\right\|_{p} \leq c_{[5.26}\left\|\Xi_{t}\right\|_{p} .
$$


REMARK 5.27. As already mentioned before, Lemma 5.26 continues the work done in [22, Proof of Proposition 2.3], but also the work done in 2, Theorem 5.1]. The main new contribution consist in the fact that using the extension of Fefferman's inequality (Corollary 5.19 we are able to get an $L_{p}$ - $L_{p}$-estimate in contrast to a weaker $L_{p}-L_{r}$-estimate for $r>p$.

Proof of lemma 5.26, Let $d \mathbb{Q}^{*}:=\lambda_{T} d \mathbb{Q}$. To distinguish between the integration with respect to $\mathbb{Q}$ and $\mathbb{Q}^{*}$, but not to overload the notation, we agree that $\|\cdot\|_{p}$ always means that we integrate with respect to $\mathbb{Q}$. By Girsanov's theorem, $\left(B_{s}^{*}\right)_{s \in[0, T]}$ with $B_{s}^{*}:=B_{s}-\int_{0}^{s} c_{r} d r$ is a standard $\mathbb{Q}^{*}$-Brownian motion. Now let us fix $t \in[0, T]$ and assume that $\left\|\Xi_{t}\right\|_{p}<\infty$, otherwise there is nothing to prove. Additionally introducing

$$
b_{s}:=\frac{f^{0}\left(s, Y_{s}^{1}, Z_{s}^{1}\right)-f^{0}\left(s, Y_{s}^{0}, Z_{s}^{1}\right)}{\Delta Y_{s}} \chi_{\left\{\Delta Y_{s} \neq 0\right\}}
$$

we get that

$$
\begin{aligned}
& \Delta Y_{t} \\
= & \Delta \xi+\int_{t}^{T} a_{s} d s+\int_{t}^{T} b_{s} \Delta Y_{s} d s+\int_{t}^{T}\left\langle c_{s}, \Delta Z_{s}\right\rangle d s-\int_{t}^{T} \Delta Z_{s} d B_{s} \\
= & \Delta \xi+\int_{t}^{T} a_{s} d s+\int_{t}^{T} b_{s} \Delta Y_{s} d s-\int_{t}^{T} \Delta Z_{s} d B_{s}^{*}
\end{aligned}
$$

where our conditions assure that all terms are well-defined. Because of

$$
\mathbb{E}_{\mathbb{Q}^{*}}\left(\int_{0}^{T}\left|\Delta Z_{s}\right|^{2} d s\right)^{\frac{1}{2}} \leq\left(\mathbb{E}_{\mathbb{Q}} \lambda_{T}^{p^{\prime}}\right)^{\frac{1}{p^{\prime}}}\left(\mathbb{E}_{\mathbb{Q}}\left(\int_{0}^{T}\left|\Delta Z_{s}\right|^{2} d s\right)^{\frac{p}{2}}\right)^{\frac{1}{p}}<\infty
$$

and the Burkholder-Davis-Gundy inequalities $\left(\int_{0}^{t} \Delta Z_{s} d B_{s}^{*}\right)_{t \in[0, T]}$ is of class DL and therefore a $\mathbb{Q}^{*}$-martingale (see [80, IV.1.7]). Applying Itô's formula implies that

$$
e^{\int_{0}^{t} b_{s} d s} \Delta Y_{t}=e^{\int_{0}^{T} b_{s} d s} \Delta \xi+\int_{t}^{T} e^{\int_{0}^{s} b_{r} d r} a_{s} d s-\int_{t}^{T} e^{\int_{0}^{s} b_{r} d r} \Delta Z_{s} d B_{s}^{*}
$$

and

$$
\Delta Y_{t}=\mathbb{E}_{\mathbb{Q}^{*}}\left(e^{\int_{t}^{T} b_{s} d s} \Delta \xi+\int_{t}^{T} e^{\int_{t}^{s} b_{r} d r} a_{s} d s \mid \mathcal{A}_{t}\right) .
$$

Using $p_{0} \in(1, p)$ we continue with

$$
\left|\Delta Y_{t}\right| \leq e^{(T-t) L_{Y}} \mathbb{E}_{\mathbb{Q}^{*}}\left(\Xi_{t} \mid \mathcal{A}_{t}\right) \leq e^{(T-t) L_{Y}} \rho\left(\mathbb{E}_{\mathbb{Q}}\left(\Xi_{t}^{p_{0}} \mid \mathcal{A}_{t}\right)\right)^{\frac{1}{p_{0}}} \text { a.s. }
$$

By Doob's maximal inequality,

$$
\left\|\sup _{s \in[t, T]}\left|\Delta Y_{s}\right|\right\|_{p} \leq c_{[33}\left\|\Xi_{t}\right\|_{p}
$$

with $c_{[33}:=e^{(T-t) L_{Y}} \rho\left(\frac{p}{p-p_{0}}\right)^{\frac{1}{p_{0}}}$. Letting

$$
\Delta f_{s}:=f^{1}(s)-f^{0}\left(s, Y_{s}^{0}, Z_{s}^{0}\right),
$$

we also have that

$$
\left|\Delta f_{s}\right| \leq\left|a_{s}\right|+\left|b_{s}\right|\left|\Delta Y_{s}\right|+\left|c_{s}\right|\left|\Delta Z_{s}\right| \leq\left|a_{s}\right|+L_{Y}\left|\Delta Y_{s}\right|+\left|c_{s}\right|\left|\Delta Z_{s}\right|
$$


and

$$
\begin{aligned}
& \int_{t}^{T}\left|\Delta Y_{s} \Delta f_{s}\right| d s \\
& \leq \int_{t}^{T}\left|\Delta Y_{s}\right|\left[\left|a_{s}\right|+L_{Y}\left|\Delta Y_{s}\right|+\left|c_{s}\right|\left|\Delta Z_{s}\right|\right] d s \\
& \leq \sup _{s \in[t, T]}\left|\Delta Y_{s}\right| \int_{t}^{T}\left|a_{s}\right| d s+L_{Y} \int_{t}^{T}\left|\Delta Y_{s}\right|^{2} d s+\int_{t}^{T}\left[\left|c_{s}\right|\left|\Delta Y_{s}\right|\left|\Delta Z_{s}\right|\right] d s \\
& \leq \frac{1}{2} \sup _{s \in[t, T]}\left|\Delta Y_{s}\right|^{2}+\frac{1}{2}\left[\int_{t}^{T}\left|a_{s}\right| d s\right]^{2} \\
& \quad+L_{Y} \int_{t}^{T}\left|\Delta Y_{s}\right|^{2} d s+\int_{t}^{T}\left[\left|c_{s}\right|\left|\Delta Y_{s}\right|\left|\Delta Z_{s}\right|\right] d s \\
& \leq \quad \Gamma^{2} \sup _{s \in[t, T]}\left|\Delta Y_{s}\right|^{2}+\frac{1}{2}\left[\int_{t}^{T}\left|a_{s}\right| d s\right]^{2}+\int_{t}^{T}\left[\left|c_{s}\right|\left|\Delta Y_{s}\right|\left|\Delta Z_{s}\right|\right] d s
\end{aligned}
$$

with $\Gamma^{2}:=\frac{1}{2}+T L_{Y}$. Now for $S_{t}(Z)^{2}:=\int_{t}^{T}\left|\Delta Z_{s}\right|^{2} d s$ and ${ }^{*} Y_{t}:=\sup _{s \in[t, T]}\left|\Delta Y_{s}\right|$ using Itô's formula, the Burkholder-Davis-Gundy inequalities (11), and Corollary 5.19 , we get that

$$
\begin{aligned}
& \left\|S_{t}(Z)\right\|_{p} \\
& \leq\left\|\left(|\Delta \xi|^{2}+2\left|\int_{t}^{T} \Delta Y_{s} \Delta Z_{s} d B_{s}\right|+2 \int_{t}^{T}\left|\Delta Y_{s} \Delta f_{s}\right| d s\right)^{\frac{1}{2}}\right\|_{p} \\
& \leq \|\left(|\Delta \xi|^{2}+2\left|\int_{t}^{T} \Delta Y_{s} \Delta Z_{s} d B_{s}\right|+2 \Gamma^{2 *} Y_{t}^{2}+\left[\int_{t}^{T}\left|a_{s}\right| d s\right]^{2}\right. \\
& \left.+2 \int_{t}^{T}\left[\left|c_{s}\left\|\Delta Y_{s}\right\| \Delta Z_{s}\right|\right] d s\right)^{\frac{1}{2}} \|_{p} \\
& \leq\left\|\Xi_{t}\right\|_{p}+\sqrt{2}\left\|\int_{t}^{T}\left[\left|c_{s}\left\|\Delta Y_{s}\right\| \Delta Z_{s}\right|\right] d s\right\|_{\frac{p}{2}}^{\frac{1}{2}}+\sqrt{2}\left\|\int_{t}^{T} \Delta Y_{s} \Delta Z_{s} d B_{s}\right\|_{\frac{p}{2}}^{\frac{1}{2}} \\
& +\sqrt{2} \Gamma\left\|* Y_{t}\right\|_{p}
\end{aligned}
$$

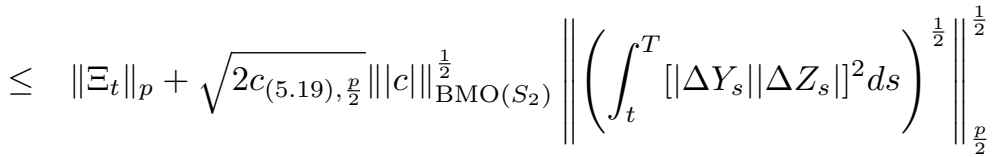

$$
\begin{aligned}
& +\sqrt{2 \beta_{p / 2}}\left\|\left(\int_{t}^{T}\left[\left|\Delta Y_{s} \| \Delta Z_{s}\right|\right]^{2} d s\right)^{\frac{1}{2}}\right\|_{\frac{p}{2}}^{\frac{1}{2}}+\sqrt{2} \Gamma\left\|* Y_{t}\right\|_{p} \\
& =\left\|\Xi_{t}\right\|_{p}+\left[\sqrt{2 c \sqrt{5.19, \frac{p}{2}}}\||c|\|_{\mathrm{BMO}\left(S_{2}\right)}^{\frac{1}{2}}+\sqrt{2 \beta_{p / 2}}\right] \times
\end{aligned}
$$




$$
\times\left\|\left(\int_{t}^{T}\left[\left|\Delta Y_{s} \| \Delta Z_{s}\right|\right]^{2} d s\right)^{\frac{1}{2}}\right\|_{\frac{p}{2}}^{\frac{1}{2}}+\sqrt{2} \Gamma\left\|* Y_{t}\right\|_{p} .
$$

Therefore, for $\kappa:=\sqrt{2 c \sqrt{5.19}, \frac{p}{2} \gamma}+\sqrt{2 \beta_{p / 2}}$ and $\lambda>0$ we obtained that

$$
\begin{aligned}
\left\|S_{t}(Z)\right\|_{p} & \leq\left\|\Xi_{t}\right\|_{p}+\kappa\left\|\left(\int_{t}^{T}\left[\left|\Delta Y_{s} \| \Delta Z_{s}\right|\right]^{2} d s\right)^{\frac{1}{2}}\right\|_{\frac{p}{2}}^{\frac{1}{2}}+\sqrt{2} \Gamma\left\|^{*} Y_{t}\right\|_{p} \\
& \leq\left\|\Xi_{t}\right\|_{p}+\kappa\left\|^{*} Y_{t} S_{t}(Z)\right\|_{\frac{p}{2}}^{\frac{1}{2}}+\sqrt{2} \Gamma\left\|^{*} Y_{t}\right\|_{p} \\
& \leq\left\|\Xi_{t}\right\|_{p}+\kappa\left\|\frac{\lambda_{2}}{2} Y_{t}^{2}+\frac{1}{2 \lambda} S_{t}(Z)^{2}\right\|_{\frac{p}{2}}^{\frac{1}{2}}+\sqrt{2} \Gamma\left\|^{*} Y_{t}\right\|_{p} \\
& \leq\left\|\Xi_{t}\right\|_{p}+\kappa \sqrt{\frac{\lambda}{2}}\left\|^{*} Y_{t}\right\|_{p}+\kappa \sqrt{\frac{1}{2 \lambda}}\left\|S_{t}(Z)\right\|_{p}+\sqrt{2} \Gamma\left\|^{*} Y_{t}\right\|_{p}
\end{aligned}
$$

Choosing $\lambda:=2 \kappa^{2}$ and using (33) gives that

$$
\begin{aligned}
\left\|S_{t}(Z)\right\|_{p} & \leq 2\left\|\Xi_{t}\right\|_{p}+\left[2 \kappa^{2}+2 \sqrt{2} \Gamma\right]\left\|{ }^{*} Y_{t}\right\|_{p} \\
& \leq 2\left\|\Xi_{t}\right\|_{p}+\left[2 \kappa^{2}+2 \sqrt{2} \Gamma\right] c_{[33}\left\|\Xi_{t}\right\|_{p}
\end{aligned}
$$

which concludes the proof. 
7 Nov 2017 05:35:26 EST

Prob+Stat

Version 2 - Submitted to Memoirs of the AMS 


\section{CHAPTER 6}

\section{Applications to BSDEs}

In this chapter we consider a solution to the BSDE

$$
Y_{t}=\xi+\int_{t}^{T} f\left(s, Y_{s}, Z_{s}\right) d s-\int_{t}^{T} Z_{s} d W_{s}, \quad t \in[0, T], \text { a.s. }
$$

and will proceed as follows: Firstly, we extend equation 34 from $(\Omega, \mathcal{F}, \mathbb{P})$ to $\left(\bar{\Omega}, \mathcal{F}^{0}, \overline{\mathbb{P}}\right)$ and follow Chapter 3 to transform this extended $\mathrm{BSDE}$ from $\left(\bar{\Omega}, \mathcal{F}^{0}, \overline{\mathbb{P}}\right)$ to $\left(\bar{\Omega}, \mathcal{F}^{\varphi}, \overline{\mathbb{P}}\right)$ and $\left(\bar{\Omega}, \mathcal{F}^{\psi}, \overline{\mathbb{P}}\right)$, respectively, and consider for $\rho \in\{\varphi, \psi\}$ the two solutions

$$
Y_{t}^{\rho}=\xi^{\rho}+\int_{t}^{T} f^{\rho}\left(s, Y_{s}^{\rho}, Z_{s}^{\rho}\right) d s-\int_{t}^{T} Z_{s}^{\rho} d W_{s}^{\rho}, \quad t \in[0, T], \text { a.s. }
$$

Therefore (35) describes two copies of (34), parametrised with $\varphi$ and $\psi$, by transforming the underlying Gaussian structure. Secondly, we interpret 35 as equations driven by the joint Brownian motion $\bar{W}=\left(\overline{W_{t}}\right)_{t \in[0, T]}$ and apply an a priori estimate to obtain Theorem 6.3 to describe the stability of (34). From the stability we obtain non-linear embeddings for Besov spaces in Section 6.4.4 and upper bounds for the $L_{p}$-variation of solution processes $(Y, Z)$ to our BSDE (34) in Section 6.5. To explain by means of Section 6.5 the usage of our general framework, let us assume for the moment that the generator $f$ in $(34)$ depends only on $(s, y, z)$. For $p \in[2, \infty)$ and $0 \leq s<t \leq T$ Theorem 6.24 provides an upper bound for $\left\|\sup _{r \in[s, t]}\left|Y_{r}-Y_{s}\right|\right\|_{p}$ that mainly depends on $\left\|\xi-\xi^{(s, t]}\right\|_{p}$. In other words, local estimates on $\xi$ imply local estimates for the variation of the process $Y$, if local is understood as local in time. To illustrate this further, assume a partition $0=r_{0}<r_{1}<\cdots<r_{L}=T$, again $p \in[2, \infty)$, and suppose for $l=1, \ldots, L$ that $\xi_{l} \in \mathcal{L}_{p}$ is a measurable functional of finitely many increments $W_{b}-W_{a}$ with $(a, b] \subseteq\left(r_{l-1}, r_{l}\right]$. Consider

$$
\xi:=g\left(\xi_{1}, \ldots, \xi_{L}\right),
$$

where $g: \mathbb{R}^{L} \rightarrow \mathbb{R}$ is a Lipschitz function with constant $L \geq 0$. Then

$$
\left\|\xi-\xi^{(s, t]}\right\|_{p} \leq L\left\|\xi_{l}-\xi_{l}^{(s, t]}\right\|_{p} \quad \text { whenever } \quad(s, t] \subseteq\left(r_{l-1}, r_{l}\right] .
$$

Therefore, the variation of $Y$ on $\left[r_{l-1}, r_{l}\right]$ is mainly determined by properties of $\xi_{l}$. This idea was first developed in [40] and then extended to the framework of Lévy processes in 41.

\subsection{The setting}

In this section we assume a stochastic basis $\left(\Omega, \mathcal{F}, \mathbb{P},\left(\mathcal{F}_{t}\right)_{t \in[0, T]}\right)$ with $\mathcal{F}=\mathcal{F}_{T}$ satisfying the usual conditions, where $\mathbb{F}=\left(\mathcal{F}_{t}\right)_{t \in[0, T]}$ is the augmentation of the natural filtration of the $d$-dimensional Brownian motion $\left(W_{t}\right)_{t \in[0, T]}$. We consider 
a solution to the BSDE (34) under the following set of assumptions, that describe the generators we will use and ensure that all expressions do exist:

\section{Assumption 6.1.}

(B1) The process $Z$ is predictable such that

$$
\mathbb{P}\left(\int_{0}^{T}\left|Z_{s}\right|^{2} d s<\infty\right)=1 .
$$

(B2) The process $Y$ is adapted and path-wise continuous.

(B3) The generator $f: \Omega_{T} \times \mathbb{R} \times \mathbb{R}^{d} \rightarrow \mathbb{R}$ is such that $(t, \omega) \mapsto f(t, \omega, y, z)$ is predictable for all $(y, z)$ and there are $L_{Y}, L_{Z} \geq 0$ and $\theta \in[0,1]$ such that

$$
\left|f\left(t, \omega, y_{0}, z_{0}\right)-f\left(t, \omega, y_{1}, z_{1}\right)\right| \leq L_{Y}\left|y_{0}-y_{1}\right|+L_{Z}\left[1+\left|z_{0}\right|+\left|z_{1}\right|\right]^{\theta}\left|z_{0}-z_{1}\right|
$$

for all $\left(t, \omega, y_{0}, y_{1}, z_{0}, z_{1}\right)$.

(B4) $\mathbb{P}\left(\int_{0}^{T}\left|f\left(s, Y_{s}, Z_{s}\right)\right| d s<\infty\right)=1$.

The case $\theta=0$ is the standard Lipschitz case, the case $\theta=1$ the standard quadratic case, and $\theta \in(0,1)$ can be seen as sub-quadratic case (see for example [28]). Our strategy for the first step is to impose in Lemma 6.2 below conditions on the gradient process $Z$ and $f(s, 0,0)$, only, but not on $\xi$, in order to verify that we deal with an $L_{p}$-solution to our BSDE. This might also help to find more general conditions on $(\xi, f)$ that ensure the existence of $L_{p}$-solutions (see Section 6.4.1 below). Our conditions on $Z$ can be verified by results from Section 6.3 below. In the following we assume that $p \in[2, \infty)$ because this assumption will be used in some steps of the proofs and because this case is more interesting with respect to the tail-behavior of $\left|Y_{t}-Y_{s}\right|$ than the case $p<2$.

Lemma 6.2. In addition to the conditions (B1)-(B4) we assume for $p \in[2, \infty)$ that

(B5) $\int_{0}^{T}|f(s, 0,0)| d s \in \mathcal{L}_{p}$,

(B6) $\left(\int_{0}^{T}\left|Z_{s}\right|^{2} d s\right)^{\frac{1}{2}} \in \mathcal{L}_{p}$

(B7) $\int_{0}^{T}\left|Z_{s}\right|^{1+\theta} d s \in \mathcal{L}_{p}$.

Then $\int_{0}^{T}\left|f\left(s, Y_{s}, Z_{s}\right)\right| d s+\sup _{t \in[0, T]}\left|Y_{t}\right| \in \mathcal{L}_{p}$.

Proof. We rewrite (34) as

$$
Y_{t}=Y_{0}-\int_{0}^{t} f\left(s, Y_{s}, Z_{s}\right) d s+\int_{0}^{t} Z_{s} d W_{s}
$$

for $t \in[0, T]$. For an integer $N \geq 1$ let

$$
\tau_{N}:=\inf \left\{t \in[0, T]:\left|Y_{t}-Y_{0}\right|=N\right\} \wedge T
$$

with $\inf \emptyset:=\infty$. Then

$$
Y_{t \wedge \tau_{N}}=Y_{0}-\int_{0}^{t \wedge \tau_{N}} f\left(s, Y_{s}, Z_{s}\right) d s+\int_{0}^{t \wedge \tau_{N}} Z_{s} d W_{s} .
$$

Because of

$$
|f(s, y, z)| \leq|f(s, 0,0)|+L_{y}|y|+L_{z}[1+|z|]^{\theta}|z|
$$


we conclude that

$$
\begin{aligned}
\left|Y_{t \wedge \tau_{N}}\right| \leq & {\left[\left|Y_{0}\right|+\int_{0}^{T}|f(s, 0,0)| d s+L_{z} \int_{0}^{T}\left[1+\left|Z_{s}\right|\right]^{\theta}\left|Z_{s}\right| d s\right.} \\
& \left.+\sup _{r \in[0, T]}\left|\int_{0}^{r} Z_{s} d W_{s}\right|\right]+L_{y} \int_{0}^{t \wedge \tau_{N}}\left|Y_{s \wedge \tau_{N}}\right| d s \\
=: & A+L_{y} \int_{0}^{t \wedge \tau_{N}}\left|Y_{s \wedge \tau_{N}}\right| d s
\end{aligned}
$$

and

$$
M_{t}^{N} \leq A+L_{y} \int_{0}^{t} M_{s}^{N} d s
$$

with

$$
M_{s}^{N}:=\sup _{r \in[0, s]}\left|Y_{r \wedge \tau_{N}}\right|=\sup _{r \in\left[0, s \wedge \tau_{N}\right]}\left|Y_{r}\right| .
$$

The process $\left(M_{t}^{N}\right)_{t \in[0, T]}$ is continuous, adapted and bounded by $\left|Y_{0}\right|+N$. The inequality

$$
\left\|M_{t}^{N}\right\|_{p} \leq\|A\|_{p}+L_{y} \int_{0}^{t}\left\|M_{s}^{N}\right\|_{p} d s
$$

implies by Gronwall's lemma that

$$
\left\|M_{T}^{N}\right\|_{p} \leq e^{L_{y} T}\|A\|_{p}
$$

Letting $N \rightarrow \infty$ gives $\sup _{t \in[0, T]}\left|Y_{t}\right| \in \mathcal{L}_{p}$ because $A \in \mathcal{L}_{p}$ which follows from conditions (B5), (B6), and (B7). Finally, using (36) the part $\int_{0}^{T}\left|f\left(s, Y_{s}, Z_{s}\right)\right| d s \in$ $\mathcal{L}_{p}$ follows.

Condition (B5) is a condition on the initial data of the BSDE, whereas (B6) and (B7) are implicit conditions on the solution. For $\theta=0$ condition (B6) implies (B7). Conversely, for $\theta=1$ condition (B7) implies (B6). A sufficient condition for both, (B6) and (B7), is $\left(\int_{0}^{T}\left|Z_{s}\right|^{2} d s\right)^{1 / 2} \in \mathcal{L}_{(1+\theta) p}$.

\subsection{Stability of BSDEs with respect to perturbations of the Gaussian structure}

Now we substantiate the procedure explained in the beginning of this chapter: we assume the setting of Section 4.2 and follow Convention 4.5 (1) to extend (34) to $\bar{\Omega}$ and find

$$
\tilde{Y}_{t}=\tilde{\xi}+\int_{t}^{T} \tilde{f}\left(s, \tilde{Y}_{s}, \tilde{Z}_{s}\right) d s-\int_{t}^{T} \tilde{Z}_{s} d W_{s}^{0}, \quad t \in[0, T] .
$$

We remark that for a $(\mathcal{P}, \mathcal{B}(C(M)))$-measurable $h:[0, T] \times \Omega \rightarrow C(M)$ the extension $\widetilde{h}:[0, T] \times \bar{\Omega} \rightarrow C(M)$ is $\left(\mathcal{P}^{0}, \mathcal{B}(C(M))\right)$-measurable, and that there is a $\bar{\Omega}_{0} \in \overline{\mathcal{F}}$ with $\overline{\mathbb{P}}\left(\bar{\Omega}_{0}\right)=1$, such that $\left(\int_{0}^{t} \widetilde{Z}_{s} d W_{s}^{0}\right)\left(\omega, \omega^{\prime}\right)=\left(\int_{0}^{t} Z_{s} d W_{s}\right)(\omega)$ for $t \in[0, T]$ and $\left(\omega, \omega^{\prime}\right) \in \bar{\Omega}_{0}$. Moreover, it is clear that the inequality from (B3) transfers directly. Therefore we assume that (34) is extended to (37) where we simplify the notation by denoting $(\tilde{\xi}, \tilde{f}, \tilde{Y}, \tilde{Z})$ again by $(\xi, f, Y, Z)$. Using Theorem 3.3 in the setting of 
Section 4.2 we obtain 35 . We also know that the transformed generator $f^{\rho}$ can be taken such that (B3) is satisfied, i.e.

$$
\begin{aligned}
& \left|f^{\rho}\left(t, \bar{\omega}, y_{0}, z_{0}\right)-f^{\rho}\left(t, \bar{\omega}, y_{1}, z_{1}\right)\right| \\
& \quad \leq L_{Y}\left|y_{0}-y_{1}\right|+L_{Z}\left[1+\left|z_{0}\right|+\left|z_{1}\right|\right]^{\theta}\left|z_{0}-z_{1}\right|=: H\left(\left(y_{0}, z_{0}\right),\left(y_{1}, z_{1}\right)\right),
\end{aligned}
$$

which follows from Remark 3.4 .

Now let us turn to our basic result. Our strategy is to impose the conditions (B1)(B6) and an extra condition on $Z$ on equation (34) in the context of the stochastic basis $\left(\Omega, \mathcal{F}, \mathbb{P},\left(\mathcal{F}_{t}\right)_{t \in[0, T]}\right)$ we did start from, and then to deduce by Lemma 5.26 the moment estimates in the extended setting of $(\bar{\Omega}, \overline{\mathbb{P}})$. For the following we remind the reader that the number $\mathrm{sl}_{N}^{S_{2}, \mathbb{A}}(c)$ for an $\mathbb{R}$-valued progressively measurable process $c, N \geq 1$, and a filtration $\mathbb{A}$ was defined in Definition 5.8 .

Theorem 6.3. Assume $\theta \in[0,1]$, for equation (34) conditions (B1)-(B4), and additionally $|Z| \in \operatorname{BMO}\left(S_{2 \theta}\right)$ in the case $\theta \in(0,1]$. Suppose that there is a nonincreasing sequence $\left(s_{N}\right)_{N \geq 1} \subseteq[0, \infty)$, where $s_{\infty}:=\lim _{N} s_{N}$, such that

$$
\mathrm{sl}_{N}^{S_{2}, \mathbb{F}}\left(|Z|^{\theta}\right) \leq s_{N} .
$$

Suppose that conditions (B5)-(B6) are satisfied for $p \in[2, \infty)$ where in the case $s_{\infty}>0$ we additionally assume that

$$
p>p_{0}:=\frac{\Phi^{-1}\left(2 \sqrt{2} L_{Z} s_{\infty}\right)}{\Phi^{-1}\left(2 \sqrt{2} L_{Z} s_{\infty}\right)-1} \in(1, \infty)
$$

with the function $\Phi$ defined in (31). Then, one has for the extended equations for all $t \in[0, T]$ that

$$
\begin{gathered}
\left\|\sup _{s \in[t, T]}\left|Y_{s}^{\varphi}-Y_{s}^{\psi}\right|\right\|_{p}+\left\|\left(\int_{t}^{T} D[\varphi(s), \psi(s)]\left|Z_{s}\right|^{2} d s\right)^{\frac{1}{2}}\right\|_{p} \\
+\left\|\left(\int_{t}^{T}\left|Z_{s}^{\varphi}-Z_{s}^{\psi}\right|^{2} d s\right)^{\frac{1}{2}}\right\|_{p} \\
\leq \quad c_{6.3]}\left[\left\|\xi^{\varphi}-\xi^{\psi}\right\|_{p}+\left\|\int_{t}^{T}\left|f^{\varphi}\left(s, Y_{s}^{\psi}, Z_{s}^{\psi}\right)-f^{\psi}\left(s, Y_{s}^{\psi}, Z_{s}^{\psi}\right)\right| d s\right\|_{p}\right],
\end{gathered}
$$

where $\varphi, \psi \in \Delta, D\left[\eta_{1}, \eta_{2}\right]:=1-\sqrt{1-\eta_{1}^{2}} \sqrt{1-\eta_{2}^{2}}-\eta_{1} \eta_{2}$, and $\left.c \sqrt[6.3]\right]{ }>0$ depends at most on $\left(L_{Y}, L_{Z}, T,\left(s_{N}\right)_{N=1}^{\infty}, p, d\right)$.

The applications of Theorem 6.3 are at least two-fold: Firstly, we obtain a non-linear embedding theorem for Besov spaces in Section 6.4.4 (Corollary 6.21). Secondly, we deduce in Section 6.5 upper bounds for the $L_{p}$-variation of solution processes $(Y, Z)$ to our BSDE (34). 


\section{REMARK 6.4.}

(1) The function $D\left[\eta_{1}, \eta_{2}\right]:[0,1]^{2} \rightarrow[0,1]$ measures the distance between $\eta_{1}$ and $\eta_{2}$, by projecting the vector $\left(\eta_{1}, \sqrt{1-\eta_{1}^{2}}\right)$ onto the linear subspace generated by $\left(\eta_{2}, \sqrt{1-\eta_{2}^{2}}\right)$, and by comparing the projection to $\left(\eta_{2}, \sqrt{1-\eta_{2}^{2}}\right)$. In particular, $D\left[\eta_{1}, \eta_{2}\right]=0$ if and only if $\eta_{1}=\eta_{2}$.

(2) Because the case $\lim _{N} s_{N}=0$ is of particular importance in Theorem 6.3. as it enables us to use the full range $p \in[2, \infty)$, we give some examples for this situation:

(a) For $\theta=0$ we have that

$$
\mathrm{sl}_{N}^{S_{2}, \mathbb{F}}\left(|Z|^{\theta}\right) \leq \sqrt{\frac{T}{N}}
$$

if we take equidistant time-nets.

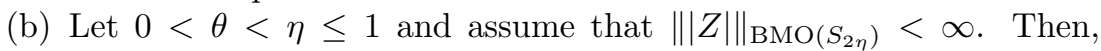
similarly to Example 5.6, we obtain

$$
\begin{aligned}
& \left\|\left(\chi_{(a, b]}(t)\left|Z_{t}\right|\right)_{t \in[0, T]}\right\|_{\mathrm{BMO}\left(S_{2 \theta}\right)} \\
\leq \quad & (b-a)^{\frac{1}{2 \theta}-\frac{1}{2 \eta}}\left\|\left(\chi_{(a, b]}(t)\left|Z_{t}\right|\right)_{t \in[0, T]}\right\|_{\operatorname{BMO}\left(S_{2 \eta}\right)}
\end{aligned}
$$

and, by using equidistant time-nets, that

$$
\mathrm{sl}_{N}^{S_{2}, \mathbb{F}}\left(|Z|^{\theta}\right) \leq\left(\frac{T}{N}\right)^{\frac{1}{2}\left(1-\frac{\theta}{\eta}\right)}\||Z|\|_{\mathrm{BMO}\left(S_{2 \eta}\right)}^{\theta} .
$$

(3) The usage of $\left(\mathrm{sl}_{N}^{S_{2}, \mathbb{F}}\left(|Z|^{\theta}\right)\right)_{N \geq 1}$ might not be optimal in extremal cases as we mainly need the reverse Hölder inequality for the Doléan-Dade exponential (44) in the proof of Theorem 6.3 below: If one would have $\int_{0}^{\cdot} c_{s} d \bar{W}_{s} \in \bar{L}_{\infty} \mathrm{BMO}_{2}$, then according to the remarks following Proposition 5.24 the reverse Hölder inequality for all exponents would be satisfied. It is part of future work to check conditions on the gradient $Z$ which guarantee this. On the other hand, if $\int_{0}^{*} c_{s} d \bar{W}_{s} \notin{\overline{L_{\infty}}}^{\mathrm{BMO}_{2}}$, then our approach yields explicit bounds for $c \sqrt{6.3}>0$ and the threshold $p_{0}$ in terms of $\left(s_{N}\right)_{N \geq 1}$ which is implicitly a novelty of this statement. As shown in Section 6.3 below, the usage of the sliceable numbers gives $s_{\infty}=0$ in our relevant cases.

(4) In 37 the sliceability condition is applied directly to $\xi$, instead of to $|Z|^{\theta}$ as in our Theorem 6.3. This is done to consider a new concept of a solution to a BSDE, called split solution, to solve multidimensional quadratic BSDEs.

Proof of Theorem 6.3. (a) By Corollary 5.21 the assumptions (B6) and $\||Z|\|_{\mathrm{BMO}\left(S_{2 \theta}\right)}<\infty$ imply (B7) in the case $\theta>0$, whereas for $\theta=0$ condition (B6) implies (B7) directly. Therefore we have

$$
\int_{0}^{T}\left|f\left(s, Y_{s}, Z_{s}\right)\right| d s+\sup _{t \in[0, T]}\left|Y_{t}\right| \in \mathcal{L}_{p}
$$

by Lemma 6.2 for equation (34). This yields the validity of conditions (B1)-(B7) and $(38)$ for the canonical extension to $\bar{\Omega}$. 
(b) Now we define $h_{1}, h_{2}:[0,1]^{2} \rightarrow[0,1]$ by

$$
\begin{aligned}
& h_{1}(x, z):=\frac{x \sqrt{1-z^{2}}+z \sqrt{1-x^{2}}}{x+z}, \\
& h_{2}(x, z):=\frac{x \sqrt{1-z^{2}}+z \sqrt{1-x^{2}}}{\sqrt{1-z^{2}}+\sqrt{1-x^{2}}},
\end{aligned}
$$

where for $x=z=0$ we set $h_{1}:=1$ and $h_{2}:=0$, analogously for $x=z=1$ we set $h_{1}:=0$ and $h_{2}:=1$, so that

$$
\left(\begin{array}{ll}
\sqrt{1-x^{2}} & x \\
\sqrt{1-z^{2}} & z
\end{array}\right)\left(\begin{array}{l}
h_{1}(x, z) \\
h_{2}(x, z)
\end{array}\right)=\left(\begin{array}{l}
1 \\
1
\end{array}\right)
$$

for all $x, z \in[0,1]$. For $\rho \in\{\varphi, \psi\}$ we let

$$
\begin{aligned}
\bar{Z}_{s}^{\rho} & :=\left(Z_{s}^{\rho} \sqrt{1-\rho^{2}(s)}, Z_{s}^{\rho} \rho(s)\right), \\
\bar{f}^{\rho}\left(s, y,\left(z, z^{\prime}\right)\right) & :=f^{\rho}\left(s, y, h_{1}(\varphi(s), \psi(s)) z+h_{2}(\varphi(s), \psi(s)) z^{\prime}\right),
\end{aligned}
$$

which leads to $\bar{f}^{\rho}\left(s, Y_{s}^{\rho}, \bar{Z}_{s}^{\rho}\right)=f^{\rho}\left(s, Y_{s}^{\rho}, Z_{s}^{\rho}\right)$ and

$$
Y_{t}^{\rho}=\xi^{\rho}+\int_{t}^{T} \bar{f}^{\rho}\left(s, Y_{s}^{\rho}, \bar{Z}_{s}^{\rho}\right) d s-\int_{t}^{T} \bar{Z}_{s}^{\rho} d \bar{W}_{s} .
$$

Observe that

$$
\begin{aligned}
& \left|\bar{Z}_{s}^{\varphi}-\bar{Z}_{s}^{\psi}\right|^{2} \\
= & D[\varphi(s), \psi(s)]\left[\left|Z_{s}^{\psi}\right|^{2}+\left|Z_{s}^{\varphi}\right|^{2}\right]+[1-D[\varphi(s), \psi(s)]]\left|Z_{s}^{\psi}-Z_{s}^{\varphi}\right|^{2} \\
\geq & \frac{2-D[\varphi(s), \psi(s)]}{2}\left|Z_{s}^{\psi}-Z_{s}^{\varphi}\right|^{2} \\
\geq & \frac{\left|Z_{s}^{\psi}-Z_{s}^{\varphi}\right|^{2}}{2}
\end{aligned}
$$

and therefore we get for

$$
\begin{aligned}
c_{s} & :=\frac{\bar{f}^{\varphi}\left(s, Y_{s}^{\varphi}, \bar{Z}_{s}^{\varphi}\right)-\bar{f}^{\varphi}\left(s, Y_{s}^{\varphi}, \bar{Z}_{s}^{\psi}\right)}{\left|\bar{Z}_{s}^{\varphi}-\bar{Z}_{s}^{\psi}\right|^{2}} \chi_{\left\{\bar{Z}_{s}^{\varphi} \neq \bar{Z}_{s}^{\psi}\right\}}\left[\bar{Z}_{s}^{\varphi}-\bar{Z}_{s}^{\psi}\right] \\
& =\frac{f^{\varphi}\left(s, Y_{s}^{\varphi}, Z_{s}^{\varphi}\right)-f^{\varphi}\left(s, Y_{s}^{\varphi}, Z_{s}^{\psi}\right)}{\left|\bar{Z}_{s}^{\varphi}-\bar{Z}_{s}^{\psi}\right|^{2}} \chi_{\left\{\bar{Z}_{s}^{\varphi} \neq \bar{Z}_{s}^{\psi}\right\}}\left[\bar{Z}_{s}^{\varphi}-\bar{Z}_{s}^{\psi}\right]
\end{aligned}
$$

that

$$
\begin{aligned}
\left|c_{s}\right| & \leq \sqrt{2} \frac{\left|f^{\varphi}\left(s, Y_{s}^{\varphi}, Z_{s}^{\varphi}\right)-f^{\varphi}\left(s, Y_{s}^{\varphi}, Z_{s}^{\psi}\right)\right|}{\left|Z_{s}^{\varphi}-Z_{s}^{\psi}\right|} \chi_{\left\{Z_{s}^{\varphi} \neq Z_{s}^{\psi}\right\}} \\
& \leq \sqrt{2} L_{Z}\left[1+\left|Z_{s}^{\psi}\right|+\left|Z_{s}^{\varphi}\right|\right]^{\theta} \\
& \leq \sqrt{2} L_{Z}\left[1+\left|Z_{s}^{\psi}\right|^{\theta}+\left|Z_{s}^{\varphi}\right|^{\theta}\right] .
\end{aligned}
$$

Lemma 5.5 (to come into the setting of Lemma 5.5 one can pass from an $\mathbb{R}$-valued progressively measurable process $\alpha=\left(\alpha_{t}\right)_{t \in[0, T]}$ with $\mathbb{E} \int_{0}^{T}\left|\alpha_{t}\right|^{2} d t<\infty$ to a martingale by, for example, $\left.M_{t}:=\int_{0}^{t} \alpha_{s} d \bar{W}_{s, 1}\right)$ gives that

$$
\mathrm{sl}_{3 N-2}^{S_{2}, \overline{\mathbb{F}}}(|c|) \leq \sqrt{2} L_{Z}\left[\mathrm{sl}_{N}^{S_{2}, \overline{\mathbb{F}}}(1)+\mathrm{sl}_{N}^{S_{2}, \overline{\mathbb{F}}}\left(\left|Z^{\psi}\right|^{\theta}\right)+\mathrm{sl}_{N}^{S_{2}, \overline{\mathbb{F}}}\left(\left|Z^{\varphi}\right|^{\theta}\right)\right] .
$$


(c) We return to the stochastic basis $\left(\Omega, \mathcal{F}, \mathbb{P},\left(\mathcal{F}_{t}\right)_{t \in[0, T]}\right)$, take $\eta>0$ and find a sequence of stopping times $0=\tau_{0} \leq \cdots \leq \tau_{N}=T$ such that

$$
\sup _{k=1, \ldots, N}\left\|\left(\chi_{\left(\tau_{k-1}, \tau_{k}\right]}(t)\left|Z_{t}\right|^{\theta}\right)_{t \in[0, T]}\right\|_{\mathrm{BMO}\left(S_{2}\right)} \leq \operatorname{sl}_{N}^{S_{2}, \mathbb{F}}\left(|Z|^{\theta}\right)+\eta \leq s_{N}+\eta .
$$

Letting

$$
Z_{t}^{k}:=\chi_{\left(\tau_{k-1}, \tau_{k}\right]}(t) Z_{t}
$$

one can quickly check that

$$
\mathbb{E}\left(\int_{t}^{T}\left|Z_{s}^{k}\right|^{2 \theta} d s \mid \mathcal{F}_{t}^{0}\right) \leq\left(s_{N}+\eta\right)^{2}
$$

for all deterministic $t \in[0, T]$, where $Z^{k}$ is canonically extended to $\bar{\Omega}$. Assuming an $\left(\mathcal{F}_{t}^{0}\right)_{t \in[0, T]}$-stopping time $\tau: \bar{\Omega} \rightarrow[0, T]$, and using the decomposition

$$
\mathbb{E}\left(\int_{\tau}^{T}\left|Z_{s}^{k}\right|^{2 \theta} d s \mid \mathcal{F}_{\tau}^{0}\right)=\mathbb{E}\left(\int_{0}^{T}\left|Z_{s}^{k}\right|^{2 \theta} d s \mid \mathcal{F}_{\tau}^{0}\right)-\int_{0}^{\tau}\left|Z_{s}^{k}\right|^{2 \theta} d s
$$

and the optional stopping theorem, we may deduce that

$$
\mathbb{E}\left(\int_{\tau}^{T}\left|Z_{s}^{k}\right|^{2 \theta} d s \mid \mathcal{F}_{\tau}^{0}\right) \leq\left(s_{N}+\eta\right)^{2} .
$$

Consequently,

$$
\sup _{k=1, \ldots, N}\left\|\left(\chi_{\left(\tau_{k-1}, \tau_{k}\right]}(t)\left|Z_{t}\right|^{\theta}\right)_{t \in[0, T]}\right\|_{\mathrm{BMO}\left(S_{2}\right)} \leq s_{N}+\eta
$$

also after extending $Z$ and $\left(\tau_{k}\right)_{k=0}^{N}$ to $\bar{\Omega}$ where the filtration $\mathbb{F}^{0}=\left(\mathcal{F}_{t}^{0}\right)_{t \in[0, T]}$ is used. This means that

$$
\mathrm{sl}_{N}^{S_{2}, \mathbb{F}^{0}}\left(|Z|^{\theta}\right) \leq s_{N}
$$

(d) For any stopping time $\tau: \Omega \rightarrow[0, T]$ relative to $\left(\Omega, \mathcal{F}, \mathbb{P},\left(\mathcal{F}_{t}\right)_{t \in[0, T]}\right)$ and for $\rho \in\{\psi, \varphi\}$ consider $\tau^{\rho}: \bar{\Omega} \rightarrow \mathbb{R}$ and take a representative such that $\tau^{\rho}: \bar{\Omega} \rightarrow[0, T]$. It is easy to check that $\tau^{\rho}$ is a stopping time with respect to the filtration $\left(\mathcal{F}_{t}^{\rho}\right)_{t \in[0, T]}$. Using $\mathbb{E}\left(A^{\rho} \mid \overline{\mathcal{F}}_{t}\right)=\left(\mathbb{E}\left(A \mid \mathcal{F}_{t}^{0}\right)\right)^{\rho} \overline{\mathbb{P}}$-a.s. for $A \in \mathcal{L}_{1}\left(\bar{\Omega}, \mathcal{F}^{0}, \overline{\mathbb{P}}\right)$ (which can be checked by taking simple $A$ that depend only on finitely many increments of the Brownian motion $W$ and then passing in $L_{1}$ to the limit), Proposition 2.5. and Remark 2.14(2) yield that

$$
\int_{t}^{T} \chi_{\left(\tau_{k-1}^{\rho}, \tau_{k}^{\rho}\right]}(s)\left|Z_{s}^{\rho}\right|^{2 \theta} d s=\left(\int_{t}^{T} \chi_{\left(\tau_{k-1}, \tau_{k}\right]}(s)\left|Z_{s}\right|^{2 \theta} d s\right)^{\rho} \overline{\mathbb{P}} \text {-a.s. }
$$

and

$$
\begin{aligned}
\mathbb{E}\left(\int_{t}^{T} \chi_{\left(\tau_{k-1}^{\rho}, \tau_{k}^{\rho}\right]}(s)\left|Z_{s}^{\rho}\right|^{2 \theta} d s \mid \overline{\mathcal{F}}_{t}\right) & =\left(\mathbb{E}\left(\int_{t}^{T} \chi_{\left(\tau_{k-1}, \tau_{k}\right]}(s)\left|Z_{s}\right|^{2 \theta} d s \mid \mathcal{F}_{t}^{0}\right)\right)^{\rho} \\
& \leq\left(s_{N}+\eta\right)^{2} .
\end{aligned}
$$

Therefore, we obtain $\mathrm{sl}_{N}^{S_{2}, \overline{\mathrm{F}}}\left(\left|Z^{\rho}\right|^{\theta}\right) \leq s_{N}+\eta$ as a complement of (43) (where we use the same optional stopping argument as in step (c)) and can continue from 42 to

$$
\mathrm{sl}_{3 N-2}^{S_{2}, \overline{\mathbb{F}}}(|c|) \leq \sqrt{2} L_{Z}\left[\sqrt{\frac{T}{N}}+2 s_{N}+2 \eta\right]
$$


and

$$
\mathrm{sl}_{3 N-2}^{S_{2}, \overline{\mathbb{F}}}(|c|) \leq \sqrt{2} L_{Z}\left[\sqrt{\frac{T}{N}}+2 s_{N}\right]
$$

by $\eta \downarrow 0$. In the case $s_{\infty}=0$ take $p_{0} \in(1,2)$, say $p_{0}:=3 / 2$, and in the case $s_{\infty}>0$, define

$$
p_{0}:=\frac{\Phi^{-1}\left(2 \sqrt{2} L_{Z} s_{\infty}\right)}{\Phi^{-1}\left(2 \sqrt{2} L_{Z} s_{\infty}\right)-1} \in(1, \infty)
$$

and $p_{1}:=\left(p+p_{0}\right) / 2$ so that

$$
1<p_{0}<p_{1}<p<\infty
$$

Let

$$
\lambda_{t}:=\exp \left(\int_{0}^{t} c_{s} d \bar{W}_{s}-\frac{1}{2} \int_{0}^{t}\left|c_{s}\right|^{2} d s\right) .
$$

We find an $N \geq 1$ such that

$$
\operatorname{sl}_{3 N-2}^{S_{2}, \overline{\mathbb{F}}}(|c|) \leq \sqrt{2} L_{Z}\left[\sqrt{\frac{T}{N}}+2 s_{N}\right]<\Phi\left(p_{1}^{\prime}\right) .
$$

This $N$ depends at most on $\left(\left(s_{N}\right)_{N=1}^{\infty}, L_{Z}, T, p\right)$. Theorem 5.25 implies that

$$
\mathcal{R H}_{p_{1}^{\prime}}(\lambda) \leq\left[\Psi\left(\sqrt{2} L_{Z}\left[\sqrt{\frac{T}{N}}+2 s_{N}\right], p_{1}^{\prime}\right)\right]^{3 N-2}<\infty
$$

with $\Psi$ taken from (32). By assumption (B6) we have that

$$
\left(\int_{0}^{T}\left|Z_{s}\right|^{2} d s\right)^{\frac{1}{2}} \in \mathcal{L}_{p}
$$

Finally, fixing $t \in[0, T]$, we can assume for this $t$ that

$$
\left\|\int_{t}^{T}\left|f^{\varphi}\left(s, Y_{s}^{\psi}, Z_{s}^{\psi}\right)-f^{\psi}\left(s, Y_{s}^{\psi}, Z_{s}^{\psi}\right)\right| d s\right\|_{p}<\infty,
$$

otherwise there is nothing to prove. So we can apply Lemma 5.26 to the equations (39) for $\rho \in\{\varphi, \psi\}$ and conclude by using (40) and 41.

\subsection{On classes of quadratic and sub-quadratic BSDEs}

In this section we present results about particular classes of quadratic and subquadratic BSDEs that might be of independent interest. At the same time we check whether we may apply Theorem 6.3 to these BSDEs and what we can say about the critical value $s_{\infty}$.

There are various articles that describe the existence and quantitative properties of solutions to BSDEs and provide comparison results. For the case $\theta=0$ the reader is referred to $[\mathbf{2 1}$ and the references therein, and for the quadratic case we refer to $[60,61,62,51,23,2,24,25,68,54,50,67,66,61$. We are mainly interested in the sub-quadratic and quadratic case, i.e. the case when $\theta \in(0,1]$. In Table 1 below we describe how we will embed these cases in the framework of this article. Table 1 should be read in the way that we first choose $(\xi, \theta, f)$, then we obtain the integrability of the gradient process $Z=\left(Z_{t}\right)_{t \in[0, T]}$ and the conclusion for $s_{\infty}=\lim _{N} s_{N}$ that are required for Theorem 6.3. In the cases where 
the uniqueness of the solution is not known there exists a solution with the stated properties. In (IV)-(V) we leave out the range for $s_{\infty}$ as we do not have general results for these cases (see Remark 6.7 below). Moreover, for (II)-(V) we need the following additional condition:

(B8) One has $\sup _{(t, \omega) \in[0, T] \times \Omega}|f(t, \omega, 0,0)|<\infty, L_{Y}>0$, and $L_{Z}>0$, where the constants $L_{Y}, L_{Z} \geq 0$ were introduced in condition (B3) of Section 6.1

\begin{tabular}{|r||l|l|l||l|l|}
\hline & $\xi$ & $\theta$ & $f$ & $|Z|$ & $s_{\infty}$ \\
\hline \hline$(\mathrm{I})$ & $\begin{array}{l}\xi \in L_{p} \\
\text { for some } p \in[2, \infty)\end{array}$ & 0 & $(\mathrm{~B} 3),(\mathrm{B} 5)$ & $\mathbb{H}_{p}\left(S_{2}\right)$ & 0 \\
\hline \hline (II) & $\xi \in \mathrm{cExp}$ & $(0,1)$ & $(\mathrm{B} 3),(\mathrm{B} 8)$ & $\begin{array}{l}\mathbb{H}_{2}\left(S_{2}\right) \cap \bigcap_{\eta \in(0,1)} \\
\mathrm{BMO}\left(S_{2 \eta}\right)\end{array}$ & 0 \\
\hline (III) & $\begin{array}{l}|\xi|_{\operatorname{cExp}(\eta, \mu)}<\infty \text { for some } \\
\eta \in(0,1], \mu>\gamma e^{\beta T}\end{array}$ & 1 & $(\mathrm{~B} 3),(\mathrm{B} 8)$ & $\begin{array}{l}\mathbb{H}_{2}\left(S_{2}\right) \cap \\
\mathrm{BMO}\left(S_{2 \eta}\right)\end{array}$ & $\begin{array}{l}{[0, \infty)} \\
\text { if } \eta=1\end{array}$ \\
\hline \hline (IV) & $\begin{array}{l}\mathbb{E} e^{\mu|\xi|}<\infty \\
\text { for some } \mu>0\end{array}$ & $(0,1)$ & $(\mathrm{B} 3),(\mathrm{B} 8)$ & $\mathrm{BMO}^{\sqrt{\Psi}}\left(S_{2}\right)$ & \\
\hline (V) & $\begin{array}{l}\mathbb{E} e^{\mu|\xi|}<\infty \\
\text { for some } \mu>\gamma e^{\beta T}\end{array}$ & 1 & (B3), (B8) & $\mathrm{BMO}^{\sqrt{\Psi}}\left(S_{2}\right)$ & \\
\hline
\end{tabular}

Table 1

The spaces $\mathrm{BMO}^{\sqrt{\Psi}}\left(S_{2}\right)$ used in (IV) and (V) are explained in Theorem 6.6 and the remark following it. We note that $|Z| \in \operatorname{BMO}^{\sqrt{\Psi}}\left(S_{2}\right)$ also implies $|Z| \in \mathbb{H}_{2}\left(S_{2}\right)$. The case (I) follows from [21, Theorem 4.2] that gives (B6) and Remark 6.4(2a) yields to $s_{\infty}=0$. In the following we verify our contribution (II)-(V).

Notation and setting. There is a series of papers dealing with the quadratic case where the terminal condition is unbounded, see [23, 24, 30, 31. Below we use the setting of the initial article [23]. For future work some extensions of [23] done in [68] might be of interest for our context as well. To use the setting of [23] we introduce constants $\alpha \geq 0$ and $\beta, \gamma>0$ such that, for all $(s, \omega) \in[0, T] \times \Omega$,

$$
|f(s, \omega, y, z)| \leq \alpha+\beta|y|+\frac{\gamma}{2}|z|^{2} \quad \text { and } \quad \alpha \geq \frac{\beta}{\gamma} .
$$

In our framework we suppose, for the remainder of this section, that condition (B8) is satisfied. Moreover, we choose $(\alpha, \beta, \gamma)$ to be

$$
\begin{aligned}
\alpha & :=\max \left\{\sup _{(t, \omega) \in \Omega \times[0, T]}|f(t, \omega, 0,0)|+L_{Z}, \frac{L_{Y}}{4 L_{Z}}\right\}, \\
\beta & :=L_{Y}, \\
\gamma & :=4 L_{Z} .
\end{aligned}
$$

As in $\left[23\right.$ we use the function $\Phi_{t}:[0, \infty) \rightarrow(0, \infty)$ given by

$$
\Phi_{t}(y):=e^{\gamma \alpha \frac{e^{\beta(T-t)}-1}{\beta}} e^{y \gamma e^{\beta(T-t)}} .
$$


Moreover, we set

$$
\mu_{T}:=\gamma e^{\beta T}>\gamma
$$

which plays the role of a critical exponent in the case $\theta=1$. Applying [23, Theorem 2 ] and inspecting its proof gives the following statement:

THEOREM $6.5([\mathbf{2 3}])$. If there exists a $\mu>\mu_{T}$ such that

$$
\mathbb{E} e^{\mu|\xi|}<\infty,
$$

then there is a solution to the BSDE 34 such that

(1) $e^{\gamma\left|Y_{t}\right|} \leq \mathbb{E}\left(\Phi_{t}(|\xi|) \mid \mathcal{F}_{t}\right)$ a.s. for $t \in[0, T]$,

(2) $|Z| \in \mathbb{H}_{2}\left(S_{2}\right)$,

(3) for $0 \leq s<t \leq T$ and $\varepsilon>0$ with $\gamma+\varepsilon<\mu$ one has

$$
\begin{aligned}
& \mathbb{E}\left(\int_{s}^{t}\left|Z_{r}\right|^{2} d r \mid \mathcal{F}_{s}\right) \leq c_{[6.5]}^{2} \mathbb{E}\left(\sup _{r \in[s, t]} e^{(\gamma+\varepsilon)\left|Y_{r}\right|} \mid \mathcal{F}_{s}\right) \text { a.s. } \\
& \text { for } c_{6.5}^{2}:=2\left[\frac{1}{\gamma^{2}}+\frac{T}{\gamma} \max \left\{\alpha, \frac{\beta}{\varepsilon}\right\}\right] .
\end{aligned}
$$

Verification of (IV)-(V). Here our main observation consists in

TheOREm 6.6. Let $\theta \in(0,1]$ and assume that $\mu>\mu_{T}$ if $\theta=1$ and $\mu>0$ if $\theta \in(0,1)$. If $\mathbb{E} e^{\mu|\xi|}<\infty$, then there is a solution to the BSDE (34) such that

$$
\mathbb{E}\left(\int_{s}^{T}\left|Z_{r}\right|^{2} d r \mid \mathcal{F}_{s}\right) \leq c_{6.6}^{2} \Psi_{s} \quad \text { with } \quad \Psi_{s}:=\mathbb{E}\left(e^{\mu|\xi|} \mid \mathcal{F}_{s}\right)
$$

for all $s \in[0, T]$ and $c \sqrt{6.6}=c(\alpha, \beta, \gamma, T, \theta, \mu) \in(0, \infty)$, where we may assume $\left(\Psi_{s}\right)_{s \in[0, T]}$ to be path-wise continuous. Moreover, for all stopping times $\tau: \Omega \rightarrow$ $[0, T], B \in \mathcal{F}_{\tau}$ of positive measure, and $\lambda, \nu>0$, one has

$$
\mathbb{P}_{B}\left(\int_{\tau}^{T}\left|Z_{r}\right|^{2} d r>\lambda \nu\right) \leq e^{1-\lambda}+\delta \mathbb{P}_{B}\left(\sup _{s \in[\tau, T]} \Psi_{s}>\frac{\nu}{D}\right),
$$

where $\mathbb{P}_{B}$ is the normalized restriction of $\mathbb{P}$ to $B, D=D(\alpha, \beta, \gamma, T, \theta, \mu)>0$, and $\delta>0$ is an absolute constant.

In the spirit of [44, Definition 1] the inequality 49 could be abbreviated by

$$
\||Z|\|_{\mathrm{BMO}^{\sqrt{\Psi}}\left(S_{2}\right)} \leq c 6.6 .
$$

Proof of Theorem 6.6. Case $\theta=1$ : We choose $\varepsilon>0$ and $p \in(1, \infty)$ such that

$$
\mu=p \mu_{T}=\frac{\gamma+\varepsilon}{\gamma} \mu_{T}
$$

which implies by $\beta>0$ that $\gamma+\varepsilon<\mu$. Assuming $0 \leq s \leq T$ and applying Theorem 6.5 gives, a.s., that

$$
\begin{aligned}
\mathbb{E}\left(\int_{s}^{T}\left|Z_{r}\right|^{2} d r \mid \mathcal{F}_{s}\right) & \leq c_{6.5]}^{2} \mathbb{E}\left(\sup _{r \in[s, T]} e^{(\gamma+\varepsilon)\left|Y_{r}\right|} \mid \mathcal{F}_{s}\right) \\
& =c_{6.5}^{2} \mathbb{E}\left(\sup _{r \in[s, T]} e^{p \gamma\left|Y_{r}\right|} \mid \mathcal{F}_{s}\right)
\end{aligned}
$$




$$
\begin{aligned}
& \leq c_{[6.5}^{2} \mathbb{E}\left(\sup _{r \in[s, T]}\left[\mathbb{E}\left(\Phi_{s}(|\xi|) \mid \mathcal{F}_{r}\right)\right]^{p} \mid \mathcal{F}_{s}\right) \\
& \leq c_{6.5 \mid}^{2}\left|\frac{p}{p-1}\right|^{p} \mathbb{E}\left(\Phi_{s}(|\xi|)^{p} \mid \mathcal{F}_{s}\right) \\
& \leq c_{6.5 \mid}^{2}\left|\frac{p}{p-1}\right|^{p} \kappa_{T}^{p} \mathbb{E}\left(e^{\mu|\xi|} \mid \mathcal{F}_{s}\right),
\end{aligned}
$$

where $\kappa_{T}:=e^{\gamma \alpha \frac{e^{\beta T}-1}{\beta}}$ and for $\left(\mathbb{E}\left(\Phi_{s}(|\xi|) \mid \mathcal{F}_{r}\right)\right)_{r \in[0, T]}$ a continuous modification is taken. Therefore, letting

$$
c^{2}=c^{2}(\alpha, \beta, \gamma, T, \mu):=c_{[6.5}^{2}\left|\kappa_{T} \frac{p}{p-1}\right|^{p},
$$

we proved

$$
\mathbb{E}\left(\int_{s}^{T}\left|Z_{r}\right|^{2} d r \mid \mathcal{F}_{s}\right) \leq c^{2} \Psi_{s} \text { a.s. }
$$

Using an optional stopping argument, this can be extended to

$$
\mathbb{E}\left(\int_{\tau}^{T}\left|Z_{r}\right|^{2} d r \mid \mathcal{F}_{\tau}\right) \leq c^{2} \Psi_{\tau} \text { a.s. }
$$

for any stopping time $\tau: \Omega \rightarrow[0, T]$. Given $\nu>0$ we get

$$
\begin{aligned}
\mathbb{P}_{B}\left(\int_{\tau}^{T}\left|Z_{r}\right|^{2} d r>3 \nu\right) & \leq \mathbb{P}_{B}\left(\int_{\tau}^{T}\left|Z_{r}\right|^{2} d r>3 c^{2} \Psi_{\tau}\right)+\mathbb{P}_{B}\left(c^{2} \Psi_{\tau}>\nu\right) \\
& \leq \frac{1}{3}+\mathbb{P}_{B}\left(c^{2} \Psi_{\tau}>\nu\right) .
\end{aligned}
$$

If we define

$$
W(B, \nu ; \tau):=\mathbb{P}\left(B \cap\left\{\sup _{r \in[\tau, T]} 3 c^{2} \Psi_{r}>\nu\right\}\right),
$$

then we can directly apply [4, Theorem 1].

Case $\theta \in(0,1)$ : This case can be considered exactly as the case $\theta=1$. In fact, with our choice of parameters $(\alpha, \beta, \gamma)$ in (46), 47), and 48) we obtain the estimate

$$
|f(s, \omega, y, z)| \leq \alpha+\beta|y|+\frac{\gamma}{2}|z|^{1+\theta} .
$$

But now, for any given $\tilde{\gamma}>0$ we find an $\tilde{\alpha} \geq 0$ such that

$$
\alpha+\frac{\gamma}{2}|z|^{1+\theta} \leq \tilde{\alpha}+\frac{\tilde{\gamma}}{2}|z|^{2}
$$

for all $z \in \mathbb{R}^{d}$. In other words, we can arrange the parameters such that $\mu>\tilde{\gamma} e^{\beta T}$ (and have an additional dependence of the constants on $\theta$ ).

REMARK 6.7. Assume equation (34) with $T=d=1, f \equiv 0$, and suppose that $\mathbb{E} e^{\mu|\xi|}<\infty$ for all $\mu>0$. Then there is a unique solution $(Y, Z)$ under the assumption $Z \in \mathbb{H}_{2}\left(S_{2}\right)$. As we may choose any $\theta \in(0,1]$, we are in the setting of (IV) and $(\mathrm{V})$. Given $\eta \in(0,1]$, we will construct a $\xi$ as above with $Z \notin \operatorname{BMO}\left(S_{2 \eta}\right)$. This means, without any additional assumptions one cannot expect results about finite 
$s_{\infty}$ in (IV) and (V) of Table 1. The construction is as follows: For $\alpha \in[1, \infty)$ we recall the definition of the Orlicz spaces $L_{\exp _{\alpha}}$ (see [10]),

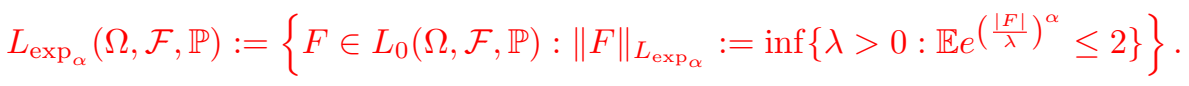

We fix $0<\eta \leq 1<\gamma<2$, determine $\alpha \in(2, \infty)$ by $\frac{1}{\gamma}=\frac{1}{\alpha}+\frac{1}{2}$, and let $t_{n}:=1-\frac{1}{2^{n}}$ for $n \geq 0$. For $\varepsilon>0, n \geq 1$, and $c_{n} \in(0, \infty)$ we set

$$
v_{n}(\omega):=2^{(n+1)\left[\frac{1}{2 \eta}+\varepsilon\right]} \chi_{\left\{\left|W_{t_{n}}(\omega)\right| \geq c_{n}\right\}}
$$

so that $\left\|v_{n}\right\|_{\infty}=2^{(n+1)\left[\frac{1}{2 \eta}+\varepsilon\right]}$. We choose $c_{n}$ such that $\left\|v_{n}\right\|_{L_{\exp _{\alpha}}} \leq 1$ and define, as in Example 5.10, the process

$$
Z_{t}:=\sum_{n=2}^{\infty} \chi_{\left(t_{n-1}, t_{n}\right]}(t) v_{n-1}
$$

The proof of Example 5.10 confirms that $Z \notin \mathrm{BMO}\left(S_{2 \eta}\right)$. On the other hand,

$$
\begin{aligned}
\left\|\int_{0}^{1} Z_{s} d W_{s}\right\|_{L_{\exp \gamma}} & \leq \sum_{n=2}^{\infty}\left\|v_{n-1}\left(W_{t_{n}}-W_{t_{n-1}}\right)\right\|_{L_{\exp _{\gamma}}} \\
& \leq \sum_{n=2}^{\infty}\left\|v_{n-1}\right\|_{L_{\exp _{\alpha}}}\left\|W_{t_{n}}-W_{t_{n-1}}\right\|_{L_{\exp _{2}}} \\
& =\left\|W_{1}\right\|_{L_{\exp _{2}}} \sum_{n=2}^{\infty}\left\|v_{n-1}\right\|_{L_{\exp _{\alpha}}} \sqrt{\frac{1}{2^{n}}} \\
& <\infty .
\end{aligned}
$$

Therefore it holds that $\xi \in L_{\exp _{\gamma}}$ with $\gamma>1$, so that $\mathbb{E} e^{\mu|\xi|}<\infty$ for all $\mu>0$.

Verification of (II)-(III). The next definition will allow us to deduce that the gradient process $Z$ belongs to $\operatorname{BMO}\left(S_{2 \eta}\right)$ :

Definition 6.8. For $\eta \in(0,1]$ and $\mu \in(0, \infty)$ we let

$$
|\xi|_{\operatorname{cExp}(\eta, \mu)}:=\sup _{t \in[0, T)}(T-t)^{\frac{1}{\eta}-1}\left\|\mathbb{E}\left(e^{\mu|\xi|} \mid \mathcal{F}_{t}\right)\right\|_{\infty} .
$$

In the notation cExp above, 'c' stands for conditional and 'Exp' for exponential.

REMARK 6.9.

(1) For $\eta=1$ we have that $|\xi|_{\operatorname{cExp}(1, \mu)}=e^{\mu\|\xi\|_{\infty}}$.

(2) For $\xi \in L_{2}, 0<\eta<\tilde{\eta}<1$, and $0<\tilde{\mu}<\mu<\infty$ with $\mu\left(\frac{1}{\tilde{\eta}}-1\right)=\tilde{\mu}\left(\frac{1}{\eta}-1\right)$ one has $|\xi|_{\operatorname{cExp}(\tilde{\eta}, \tilde{\mu})}^{\mu} \leq|\xi|_{\operatorname{cExp}(\eta, \mu)}^{\tilde{\mu}}$.

(3) For $\xi \in L_{2}$ and $\eta_{0}, \eta_{1} \in(0,1)$ one has $|\xi|_{\operatorname{cExp}\left(\eta_{0}, \mu_{0}\right)}<\infty$ for some $\mu_{0} \in$ $(0, \infty)$ if and only if $|\xi|_{c \operatorname{Exp}\left(\eta_{1}, \mu_{1}\right)}<\infty$ for some $\mu_{1} \in(0, \infty)$.

Proof. Part (1) is obvious, (3) follows directly from (2). The assertion (2) is a consequence of

$$
\begin{aligned}
|\xi|_{\operatorname{cEx}(\tilde{\eta}, \tilde{\mu})} & =\sup _{t \in[0, T]}(T-t)^{\frac{1}{\tilde{\eta}}-1}\left\|\mathbb{E}\left(e^{\tilde{\mu}|\xi|} \mid \mathcal{F}_{t}\right)\right\|_{\infty} \\
& =\sup _{t \in[0, T]}(T-t)^{\frac{1}{\tilde{\eta}}-1}\left\|\mathbb{E}\left(e^{\mu \frac{\tilde{\mu}}{\mu}|\xi|} \mid \mathcal{F}_{t}\right)\right\|_{\infty}
\end{aligned}
$$




$$
\begin{aligned}
& \leq \sup _{t \in[0, T]}(T-t)^{\frac{1}{\tilde{\eta}}-1}\left\|\mathbb{E}\left(e^{\mu|\xi|} \mid \mathcal{F}_{t}\right)\right\|_{\infty}^{\frac{\tilde{\mu}}{\mu}} \\
& =\left[\sup _{t \in[0, T]}(T-t)^{\frac{1}{\eta}-1}\left\|\mathbb{E}\left(e^{\mu|\xi|} \mid \mathcal{F}_{t}\right)\right\|_{\infty}\right]^{\frac{\tilde{\mu}}{\mu}} \\
& =|\xi|_{\operatorname{cxp}(\eta, \mu)}^{\frac{\tilde{\mu}}{\mu}}
\end{aligned}
$$

Directly from Theorem 6.6 we deduce

COROllary 6.10. Assume $\theta=1, \eta \in(0,1]$, and in addition to the assumptions made in Theorem 6.6 that $|\xi|_{\operatorname{cxp}(\eta, \mu)}<\infty$ for some $\mu \in(0, \infty)$. Then $|Z| \in$ $\operatorname{BMO}\left(S_{2 \eta}\right)$ with

$$
\||Z|\|_{\mathrm{BMO}\left(S_{2 \eta}\right)} \leq c_{\underline{6.6}}|\xi|_{\mathrm{CExp}(\eta, \mu)}^{\frac{1}{2}} .
$$

Proof. We simply have that

$$
\mathbb{E}\left(\int_{s}^{T}\left|Z_{r}\right|^{2} d r \mid \mathcal{F}_{s}\right) \leq c_{\left[\sigma_{6.6}^{2}\right.}^{2} \mathbb{E}\left(e^{\mu|\xi|} \mid \mathcal{F}_{s}\right) \leq c_{[6.6]}^{2}|\xi|_{\operatorname{cExp}(\eta, \mu)}(T-s)^{1-\frac{1}{\eta}} \text { a.s. }
$$

for all $s \in[0, T]$ and therefore, a.s.,

$$
\mathbb{E}\left(\left(\int_{s}^{T}\left|Z_{r}\right|^{2 \eta} d r\right)^{\frac{1}{\eta}} \mid \mathcal{F}_{s}\right) \leq(T-s)^{\frac{1}{\eta}-1} \mathbb{E}\left(\int_{s}^{T}\left|Z_{r}\right|^{2} d r \mid \mathcal{F}_{s}\right) \leq c^{2} \sqrt{6.6}|\xi|_{\operatorname{Exp}(\eta, \mu)} .
$$

The above corollary explains the case (III) from Table 1 . It turns out that in the remaining case (II) the particular choice of parameter $\eta$ in $|\cdot|_{\operatorname{Exp}(\eta, \mu)}$ does not have an impact. This is reflected by the following notation:

DEFINITION 6.11.

(1) For a càdlàg process $Y=\left(Y_{t}\right)_{t \in[0, T]}$ and $(\eta, \mu) \in(0,1) \times(0, \infty)$ we let

$$
|Y|_{\operatorname{cExp}(\eta, \mu)}:=\sup _{t \in[0, T)}(T-t)^{\frac{1}{\eta}-1}\left\|\mathbb{E}\left(e^{\mu \sup _{s \in[t, T]}\left|Y_{s}\right|} \mid \mathcal{F}_{t}\right)\right\|_{\infty} .
$$

We say that $Y \in \mathrm{cExp}$ provided that $|Y|_{\operatorname{cExp}(\eta, \mu)}<\infty$ for some $(\eta, \mu) \in$ $(0,1) \times(0, \infty)$.

(2) We say $\xi \in \mathrm{cExp}$ provided that $|\xi|_{\mathrm{c} \operatorname{Exp}(\eta, \mu)}<\infty$ for some $(\eta, \mu) \in(0,1) \times$ $(0, \infty)$.

The definition of $|Y|_{\operatorname{cExp}(\eta, \mu)}$ is consistent with Definition 6.8 as for a random variable $\xi$ we may let $Y_{t}:=\xi$ and get $|Y|_{\operatorname{cExp}(\eta, \mu)}=|\xi|_{\mathrm{cExp}(\eta, \mu)}$.

REMARK 6.12. Exactly as in Remark 6.9 one can show that for $\eta_{0}, \eta_{1} \in(0,1)$ one has $|Y|_{\operatorname{cExp}\left(\eta_{0}, \mu_{0}\right)}<\infty$ for some $\mu_{0} \in(0, \infty)$ if and only if $|Y|_{\operatorname{cExp}\left(\eta_{1}, \mu_{1}\right)}<\infty$ for some $\mu_{1} \in(0, \infty)$. Therefore, $Y \in \mathrm{cExp}$ if and only if there is some $\mu \in(0, \infty)$ such that

$$
\sup _{t \in[0, T)}(T-t)\left\|\mathbb{E}\left(e^{\mu \sup _{s \in[t, T]}\left|Y_{s}\right|} \mid \mathcal{F}_{t}\right)\right\|_{\infty}<\infty .
$$


Theorem 6.13. Assume that $\theta \in(0,1)$ and $\xi \in \mathrm{cExp}$. Then there is a unique solution $(Y, Z)$ to the BSDE (34) in the class where $Y \in \operatorname{cExp}$ and $|Z| \in \mathbb{H}_{2}\left(S_{2}\right)$. Moreover, for this solution we have that

(1) $s_{\infty}=0$ for $s_{\infty}$ defined as in Theorem 6.3 .

(2) $|Z| \in \operatorname{BMO}\left(S_{2 \eta}\right)$ for all $\eta \in(0,1)$.

For the uniqueness in the above theorem we do not assume convexity properties of the generator. Instead of that, we use $|Z| \in \operatorname{BMO}\left(S_{2 \theta}\right)$ and follow the methodology that BMO-properties of the $Z$ process give uniqueness, see for example [51. The difference to previous settings is that we exploit that the generator is subquadratic and get therefore a weaker condition than the standard BMO-condition $|Z| \in \operatorname{BMO}\left(S_{2}\right)$. Note that according to Example 5.10 the spaces $\operatorname{BMO}\left(S_{2 \eta}\right)$ do not coincide for different $\eta \in(0,1]$ in general.

Proof of Theorem 6.13. Existence: The condition $\xi \in \mathrm{cExp}$ implies that there are $(\eta, \mu) \in(0,1) \times(0, \infty)$ such that

$$
|\xi|_{\operatorname{cExp}(\eta, \mu)}=\sup _{t \in[0, T)}(T-t)^{\frac{1}{\eta}-1}\left\|\mathbb{E}\left(e^{\mu|\xi|} \mid \mathcal{F}_{t}\right)\right\|_{\infty}<\infty .
$$

Because of $\theta<1$ we use the argument for the case $\theta \in(0,1)$ from the proof of Theorem 6.6 to replace $(\alpha, \beta, \gamma)$ by $(\tilde{\alpha}, \beta, \tilde{\gamma})$ such that

$$
\mu>\tilde{\mu}_{T}:=\tilde{\gamma} e^{\beta T}>\tilde{\gamma}
$$

We apply Theorem 6.5 and obtain a solution with

(1) $e^{\tilde{\gamma}\left|Y_{t}\right|} \leq \mathbb{E}\left(\tilde{\Phi}_{t}(|\xi|) \mid \mathcal{F}_{t}\right)$ a.s. for $t \in[0, T]$,

(2) $|Z| \in \mathbb{H}_{2}\left(S_{2}\right)$,

where $\tilde{\Phi}_{t}$ is defined as $\Phi_{t}$ with $(\alpha, \beta, \gamma)$ replaced by $(\tilde{\alpha}, \beta, \tilde{\gamma})$. Let $\tilde{p}:=\mu / \tilde{\mu}_{T} \in(1, \infty)$ and assume $\tilde{\gamma}+\varepsilon<\mu$ for some $\varepsilon>0$. Assuming $s \in[0, T)$, the arguments from the proof of Theorem 6.6 give, a.s., that

$$
\begin{aligned}
\mathbb{E}\left(\sup _{r \in[s, T]} e^{(\tilde{\gamma}+\varepsilon)\left|Y_{r}\right|} \mid \mathcal{F}_{s}\right) & \leq\left|\frac{\tilde{p}}{\tilde{p}-1}\right|^{\tilde{p}} \tilde{\kappa}_{T}^{\tilde{p}} \mathbb{E}\left(e^{\mu|\xi|} \mid \mathcal{F}_{s}\right) \\
& \leq\left|\frac{\tilde{p}}{\tilde{p}-1}\right|^{\tilde{p}} \tilde{\kappa}_{T}^{\tilde{p}}|\xi|_{\operatorname{cExp}(\eta, \mu)}(T-s)^{1-\frac{1}{\eta}}
\end{aligned}
$$

where $\tilde{\kappa}_{T}:=e^{\tilde{\gamma} \tilde{\alpha} \frac{e^{\beta T}-1}{\beta}}$. Therefore, $|Y|_{\operatorname{cExp}(\eta, \tilde{\gamma}+\varepsilon)}<\infty$ and $Y \in \mathrm{cExp}$.

Uniqueness: Assume two solutions $\left(Y^{0}, Z^{0}\right)$ and $\left(Y^{1}, Z^{1}\right)$ with $Y^{0}, Y^{1} \in \mathrm{cExp}$ and $\overline{Z^{0}, Z^{1} \in \mathbb{H}_{2}}\left(S_{2}\right)$. Let us fix $\eta \in(0,1)$ and find $\mu_{0}, \mu_{1} \in(0, \infty)$ such that

$$
\left|Y^{i}\right|_{\operatorname{cExp}\left(\eta, \mu_{\mathrm{i}}\right)}=\sup _{t \in[0, T)}(T-t)^{\frac{1}{\eta}-1}\left\|\mathbb{E}\left(e^{\mu_{i} \sup _{s \in[t, T]}\left|Y_{s}^{i}\right|} \mid \mathcal{F}_{t}\right)\right\|_{\infty}<\infty .
$$

Again exploiting $\theta<1$, we change in 45 the parameters $(\alpha, \beta, \gamma)$ to $(\tilde{\alpha}, \beta, \tilde{\gamma})$ such that

$$
\mu:=\min \left\{\mu_{0}, \mu_{1}\right\}>\tilde{\gamma} e^{\beta T} .
$$

Analyzing the proof of [23. Theorem 2, pp. 609-610] gives for $0 \leq s<T$ and $\varepsilon>0$ with $\tilde{\gamma}+\varepsilon<\mu$ that

$$
\mathbb{E}\left(\int_{s}^{T}\left|Z_{r}^{i}\right|^{2} d r \mid \mathcal{F}_{s}\right) \leq 2\left[\frac{1}{\tilde{\gamma}^{2}}+\frac{T}{\tilde{\gamma}} \max \left\{\tilde{\alpha}, \frac{\tilde{\beta}}{\varepsilon}\right\}\right] \mathbb{E}\left(\sup _{r \in[s, T]} e^{(\tilde{\gamma}+\varepsilon)\left|Y_{r}^{i}\right|} \mid \mathcal{F}_{s}\right) \text { a.s. }
$$


We continue with

$$
\mathbb{E}\left(\sup _{r \in[s, t]} e^{(\tilde{\gamma}+\varepsilon)\left|Y_{r}^{i}\right|} \mid \mathcal{F}_{s}\right) \leq \mathbb{E}\left(\sup _{r \in[s, t]} e^{\mu_{i}\left|Y_{r}^{i}\right|} \mid \mathcal{F}_{s}\right) \leq\left|Y^{i}\right|_{\operatorname{cExp}\left(\eta, \mu_{\mathrm{i}}\right)}(T-s)^{1-\frac{1}{\eta}}
$$

Therefore, for $\tilde{c}^{2}:=2\left[\frac{1}{\tilde{\gamma}^{2}}+\frac{T}{\tilde{\gamma}} \max \left\{\tilde{\alpha}, \frac{\tilde{\beta}}{\varepsilon}\right\}\right]$, a.s.,

$$
\mathbb{E}\left(\left(\int_{s}^{T}\left|Z_{r}^{i}\right|^{2 \eta} d r\right)^{\frac{1}{\eta}} \mid \mathcal{F}_{s}\right) \leq(T-s)^{\frac{1}{\eta}-1} \mathbb{E}\left(\int_{s}^{T}\left|Z_{r}^{i}\right|^{2} d r \mid \mathcal{F}_{s}\right) \leq \tilde{c}^{2}\left|Y^{i}\right|_{\operatorname{cExp}\left(\eta, \mu_{\mathrm{i}}\right)} .
$$

This implies that $Z^{0}, Z^{1} \in \operatorname{BMO}\left(S_{2 \eta}\right)$ for all $\eta \in(0,1)$. In particular, we have that $Z^{0}, Z^{1} \in \operatorname{BMO}\left(S_{2 \theta}\right)$ and this enables us to apply Lemma 5.26. Here we set

$$
\begin{aligned}
f^{0}(s, y, z) & :=f(s, y, z), \\
f^{1}(s) & :=f\left(s, Y_{s}^{1}, Z_{s}^{1}\right) .
\end{aligned}
$$

The assumptions (D1), (D2), and (D4) are obviously satisfied, for (D3) we use that

$$
\left(\left.\left.\mathbb{E}\left|\int_{0}^{T}\right| Z_{s}^{i}\right|^{1+\theta} d s\right|^{2}\right)^{\frac{1}{2}} \leq c_{[5.19,2}\left\|\left|Z^{i}\right|\right\|_{\mathbb{H}_{2}\left(S_{2}\right)}\left\|\left|Z^{i}\right|^{\theta}\right\|_{\mathrm{BMO}\left(S_{2}\right)}
$$

where $\left\|\left|Z^{i}\right|^{\theta}\right\|_{\operatorname{BMO}\left(S_{2}\right)}<\infty$ because of $\left|Z^{i}\right| \in \operatorname{BMO}\left(S_{2 \theta}\right)$. The above definitions guarantee that $\Xi_{s} \equiv 0$. A straightforward computation gives also that

$$
\mathbb{E}\left(\int_{t}^{T}\left|c_{s}\right|^{2} d s \mid \mathcal{F}_{t}\right) \leq L_{Z}^{2} 3^{2 \theta}\left[T+\left\|\left|Z^{0}\right|\right\|_{\mathrm{BMO}\left(S_{2 \theta}\right)}^{2 \theta}+\left\|\left|Z^{1}\right|\right\|_{\mathrm{BMO}\left(S_{2 \theta}\right)}^{2 \theta}\right] \text { a.s. }
$$

so that $\|c\|_{\mathrm{BMO}\left(S_{2}\right)}<\infty$. It remains to show that $p_{0}$ can be chosen such that $p_{0} \in(1,2)$. Here we repeat the above argument and check, for $0 \leq a<b \leq T$ and $\eta \in(\theta, 1)$, that

$$
\begin{aligned}
& \mathbb{E}\left(\int_{a}^{b}\left|c_{s}\right|^{2} d s \mid \mathcal{F}_{a}\right) \\
& \quad \leq L_{Z}^{2} 3^{2 \theta}\left[(b-a)+(b-a)^{1-\frac{\theta}{\eta}}\left[\left\|\left|Z^{0}\right|\right\|_{\mathrm{BMO}\left(S_{2 \eta}\right)}^{2 \theta}+\left\|\left|Z^{1}\right|\right\|_{\mathrm{BMO}\left(S_{2 \eta}\right)}^{2 \theta}\right)\right] \text { a.s. }
\end{aligned}
$$

This yields $\lim _{N} \mathrm{sl}_{N}^{S_{2}}(c)=0$ and we can choose $p_{0} \in(1,2)$. Therefore we may apply Lemma 5.26 with $p=2$ and this yields uniqueness.

The conclusion $s_{\infty}=0$ follows by Remark $6.4(2 \mathrm{~b})$, which is the same reasoning as used for $\lim _{N} \mathrm{sl}_{N}^{S_{2}}(c)=0$ above.

REMARK 6.14. Theorem 6.13 is an extension of the known case $\theta=1$ (cf. [51, 68] ). For $\theta=1$ and $\xi \in L_{\infty}$ Theorem 6.5 gives a solution $(Y, Z)$ with $\sup _{t \in[0, T]}\left\|Y_{t}\right\|_{\infty}<$ $\infty$ and $|Z| \in \operatorname{BMO}\left(S_{2}\right)$. Assuming two such solutions, we may follow the (second half of the) part about uniqueness in the proof of Theorem 6.13. Here the difference is that we only get some $p_{0} \in(1, \infty)$ for applying Lemma 5.26. However, $\left|Z^{0}-Z^{1}\right| \in$ $\operatorname{BMO}\left(S_{2}\right)$ implies that all moments of $\int_{0}^{T}\left|Z_{s}^{0}-Z_{s}^{1}\right|^{2} d s$ exist and Lemma 5.26 is applicable for any $p \in\left(p_{0}, \infty\right) \cap[2, \infty)$. Therefore, in the case $\theta=1$ and $\xi \in L_{\infty}$ the solution $(Y, Z)$ is unique when $\sup _{t \in[0, T]}\left\|Y_{t}\right\|_{\infty}<\infty$ and $|Z| \in \operatorname{BMO}\left(S_{2}\right)$. 
We finish by an example illustrating $\xi \in \mathrm{cExp}$.

EXAmPle 6.15. Let $d=1, \eta \in(0,1)$,

$$
\varphi_{\eta}(t):=\log \left(1+(T-t)^{1-\frac{1}{\eta}}\right) \quad \text { for } t \in[0, T),
$$

so that $\varphi_{\eta}(t) \uparrow \infty$ as $t \rightarrow T$ and define the stopping time

$$
\tau_{\eta}:=\inf \left\{t \in[0, T): W_{t}=\varphi_{\eta}(t)\right\} \wedge T .
$$

Let

$$
e^{\xi}:=1+e^{W_{\tau_{\eta}}-\frac{\tau_{\eta}}{2}}
$$

so that $\xi(\omega) \in(0, \infty)$ and

$$
\mathbb{E}\left(e^{\xi} \mid \mathcal{F}_{t}\right)=1+e^{W_{\tau_{\eta} \wedge t}-\frac{\tau_{\eta} \wedge t}{2}} \leq 2+(T-t)^{1-\frac{1}{\eta}} \text { a.s. }
$$

for $t \in[0, T)$. On the other hand, $\xi \notin L_{\infty}$ because for all $c>0$ one has that $\mathbb{P}\left(W_{\tau_{\eta}}>\right.$ $c)>0$. The latter fact can be checked by taking any $0<\varepsilon<\varphi_{\eta}(0)<c<\infty$ and $S \in(0, T)$ with $c<\varphi_{\eta}(S)$ and using the known fact that $\mathbb{P}\left(\sup _{t \in[0, S]}\left|W_{t}\right| \leq \varepsilon\right)>0$ so that the probability that the Brownian motion exceeds $\varphi_{\eta}$ on $[S,(S+T) / 2]$ is positive.

\subsection{Settings for the stability theorem}

The aim of this section is to discuss some settings for the stability Theorem 6.3

6.4.1. Forward setting. This setting corresponds to the setting of stochastic integration. If the generator $f$ does not depend on $Y$, then the process $Y$ computes directly as

$$
Y_{t}=Y_{0}-\int_{0}^{t} f\left(s, Z_{s}\right) d s+\int_{0}^{t} Z_{s} d W_{s} .
$$

This enables us to construct examples to understand what the correct conditions on $Z$ in the quadratic case might be. Let us mention two cases:

(a) Taking $Z$ from Example 5.10 for $0<\theta<\eta=1$, we have examples where the $Z$-process fails to be in $\operatorname{BMO}\left(S_{2}\right)$ but satisfies $Z \in \operatorname{BMO}\left(S_{2 \theta}\right)$ and $\int_{0}^{T}\left|Z_{s}\right|^{2} d s \in$ $L_{\text {exp }}$. The latter enables us to apply Lemma 6.2 under suitable integrability conditions on $\int_{0}^{T}|f(s, 0)| d s$ (note that $L_{\exp } \subseteq L_{p}$ for all $p \in(0, \infty)$ ).

(b) Similarly, for $\theta=1$ we obtain an $L_{p}$-solution of our BSDE under (B3), (B5), and $\left(\int_{0}^{T}\left|Z_{t}\right|^{2} d t\right)^{\frac{1}{2}} \in L_{2 p}$ (see the arguments at the end of Section 6.1). Therefore we can take any $Z \in \mathrm{BMO}\left(S_{2}\right)$, in particular, $Z$ can be an unbounded BMOprocess in the quadratic setting.

6.4.2. Potential estimates for the generator. In applications of Theorem 6.3 one might need to estimate

$$
\left\|\int_{t}^{T}\left|f^{\varphi}\left(s, Y_{s}^{\psi}, Z_{s}^{\psi}\right)-f^{\psi}\left(s, Y_{s}^{\psi}, Z_{s}^{\psi}\right)\right| d s\right\|_{p}
$$

from above. One way to do this (we do not consider the remaining assumptions for Theorem 6.3 is to find a potential estimate

$$
\left|f^{\varphi}(s, y, z)-f^{\psi}(s, y, z)\right| \leq\left|\left\langle\left(1,|y|,|z|,|z|^{1+\theta}\right), V_{s}^{\varphi}-V_{s}^{\psi}\right\rangle\right|
$$


for all $(s, y, z)$ where the potential $\left(V_{s}\right)_{s \in[0, T]}$ is a predictable process

$$
V_{s}: \Omega \rightarrow \mathbb{R}^{4} \text {. }
$$

Below we illustrate some special cases for $V$. The general construction is as follows: We consider a continuous

$$
h:[0, T] \times \mathbb{R}^{N} \times \mathbb{R} \times \mathbb{R}^{d} \rightarrow \mathbb{R},
$$

where $N \geq 1$, and a predictable $\mathbb{R}^{N}$-valued process $A=\left(A_{t}\right)_{t \in[0, T]}$ on $\Omega$ to let

$$
f(t, \omega, y, z):=h\left(t, A_{t}(\omega), y, z\right) .
$$

Then $f$ is $\left(\mathcal{P}, \mathcal{B}\left(C\left(\mathbb{R}^{1+d}\right)\right)\right)$-measurable. Assume that $A^{\varphi}=\left(A_{t}^{\varphi}\right)_{t \in[0, T]}$ is a $\mathcal{P}^{\varphi}$ measurable representative of $\widetilde{A}^{\varphi}$, where $\widetilde{A}$ is the canonical extension of $A$ to $\bar{\Omega}$. We get that

$$
f^{\varphi}(t, \bar{\omega}, y, z):=h\left(t, A_{t}^{\varphi}(\bar{\omega}), y, z\right)
$$

is $\left(\mathcal{P}^{\varphi}, \mathcal{B}\left(C\left(\mathbb{R}^{1+d}\right)\right)\right)$-measurable and, for any fixed $(y, z) \in \mathbb{R}^{1+d}$, that $f^{\varphi}(\cdot, \cdot, y, z)$ : $[0, T] \times \bar{\Omega} \rightarrow \mathbb{R}$ is a representative of $\tilde{f}^{\varphi}$, where $\widetilde{f}$ is the canonical extension $\widetilde{f}(\cdot, \cdot, y, z):[0, T] \times \bar{\Omega} \rightarrow \mathbb{R}$ (see Proposition 2.5(4) applied to $X_{t, 1}=t$ and $\left.\left(X_{t, 2}(\bar{\omega}), \ldots, X_{t, N+1}(\bar{\omega})\right)=\widetilde{A}_{t}(\bar{\omega})\right)$. Therefore we will take in the sequel as transformed generator the map $f^{\varphi}$ as defined above.

EXAMPLE 6.16. Let

$$
f(s, \omega, y, z):=h\left(s, A_{s}(\omega), y, z\right),
$$

where $h:[0, T] \times \mathbb{R} \times \mathbb{R} \times \mathbb{R}^{d} \rightarrow \mathbb{R}$ is continuous with

$\left|h\left(t, x_{0}, y_{0}, z_{0}\right)-h\left(t, x_{1}, y_{1}, z_{1}\right)\right| \leq L_{X}\left|x_{0}-x_{1}\right|+L_{Y}\left|y_{0}-y_{1}\right|+L_{Z}\left[1+\left|z_{0}\right|+\left|z_{1}\right|\right]\left|z_{0}-z_{1}\right|$ for all $\left(t, x_{0}, x_{1}, y_{0}, y_{1}, z_{0}, z_{1}\right)$ and $\left(A_{t}\right)_{t \in[0, T]}$ is a predictable process. Then we get

$$
\left|f^{\varphi}(s, y, z)-f^{\psi}(s, y, z)\right| \leq L_{X}\left|A_{s}^{\varphi}-A_{s}^{\psi}\right| \quad \text { and } \quad V_{s}:=\left(L_{X} A_{s}, 0,0,0\right)
$$

and

$$
\left\|\int_{t}^{T}\left|f^{\varphi}\left(s, Y_{s}^{\psi}, Z_{s}^{\psi}\right)-f^{\psi}\left(s, Y_{s}^{\psi}, Z_{s}^{\psi}\right)\right| d s\right\|_{p} \leq L_{X}\left\|\int_{t}^{T}\left|A_{s}^{\varphi}-A_{s}^{\psi}\right| d s\right\|_{p} .
$$

The next example indicates the case of random Lipschitz constants for $y$ :

EXAMPle 6.17. Assume that

$$
f(s, \omega, y, z):=A_{s}(\omega) g(y)
$$

where $g: \mathbb{R} \rightarrow \mathbb{R}$ is a Lipschitz function and $\left(A_{s}\right)_{s \in[0, T]}$ is predictable and uniformly bounded in $(s, \omega)$. Then

$$
\left|f^{\varphi}(s, y, z)-f^{\psi}(s, y, z)\right| \leq|g(y)|\left|A_{s}^{\varphi}-A_{s}^{\psi}\right| \leq[|g(0)|+\operatorname{Lip}(g)|y|]\left|A_{s}^{\varphi}-A_{s}^{\psi}\right|
$$

and $V_{s}:=\left(|g(0)| A_{s}, \operatorname{Lip}(g) A_{s}, 0,0\right)$. Here we get (for example) that

$$
\begin{aligned}
& \left\|\int_{t}^{T}\left|f^{\varphi}\left(s, Y_{s}^{\psi}, Z_{s}^{\psi}\right)-f^{\psi}\left(s, Y_{s}^{\psi}, Z_{s}^{\psi}\right)\right| d s\right\|_{p} \\
\leq & |g(0)|\left\|\int_{t}^{T}\left|A_{s}^{\varphi}-A_{s}^{\psi}\right| d s\right\|_{p}+\operatorname{Lip}(g)\left\|\int_{t}^{T}\left|Y_{s}^{\psi}\right|\left|A_{s}^{\varphi}-A_{s}^{\psi}\right| d s\right\|_{p}
\end{aligned}
$$




$$
\begin{aligned}
& \leq|g(0)|\left\|\int_{t}^{T}\left|A_{s}^{\varphi}-A_{s}^{\psi}\right| d s\right\|_{p}+\operatorname{Lip}(g)\left\|*\left(Y^{\psi}\right)_{t} \int_{t}^{T}\left|A_{s}^{\varphi}-A_{s}^{\psi}\right| d s\right\|_{p} \\
& \leq|g(0)|\left\|\int_{t}^{T}\left|A_{s}^{\varphi}-A_{s}^{\psi}\right| d s\right\|_{p}+\operatorname{Lip}(g)\left\|^{*}\left(Y^{\psi}\right)_{t}\right\|_{p_{0}}\left\|\int_{t}^{T}\left|A_{s}^{\varphi}-A_{s}^{\psi}\right| d s\right\|_{p_{1}} \\
& \leq\left[|g(0)|+\operatorname{Lip}(g)\left\|^{*} Y_{t}\right\|_{p_{0}}\right]\left\|\int_{t}^{T}\left|A_{s}^{\varphi}-A_{s}^{\psi}\right| d s\right\| \|_{p_{1}}
\end{aligned}
$$

for any $\frac{1}{p}=\frac{1}{p_{0}}+\frac{1}{p_{1}}$ with $p<p_{0}, p_{1}<\infty$ and

$$
{ }^{*} C_{t}:=\sup _{s \in[t, T]}\left|C_{s}\right|,
$$

where we used that ${ }^{*}\left(Y^{\psi}\right)_{t}$ and ${ }^{*} Y_{t}$ have the same distribution which follows from Proposition 2.5(3).

The last example concerns the $Z$ component.

EXAmple 6.18. Assume that

$$
f(s, \omega, y, z):=A_{s}(\omega)|z|^{1+\theta}
$$

with $\theta \in(0,1)$, where $\left(A_{s}\right)_{s \in[0, T]}$ is predictable and uniformly bounded in $(s, \omega)$. Then

$$
\left|f^{\varphi}(s, y, z)-f^{\psi}(s, y, z)\right| \leq|z|^{1+\theta}\left|A_{s}^{\varphi}-A_{s}^{\psi}\right|
$$

and $V_{s}:=\left(0,0,0, A_{s}\right)$. Because of

$$
\begin{aligned}
\left|f\left(s, \omega, y_{0}, z_{0}\right)-f\left(s, \omega, y_{1}, z_{1}\right)\right| & \leq\left.\left|A_{s}(\omega)\right||| z_{0}\right|^{1+\theta}-\left|z_{1}\right|^{1+\theta} \mid \\
& \leq[1+\theta]\left|A_{s}(\omega)\right|\left|z_{0}\right|-\left|z_{1}\right| \mid\left[1+\left|z_{0}\right|+\left|z_{1}\right|\right]^{\theta} \\
& \leq[1+\theta]\left|A_{s}(\omega)\right|\left|z_{0}-z_{1}\right|\left[1+\left|z_{0}\right|+\left|z_{1}\right|\right]^{\theta}
\end{aligned}
$$

the condition (B3) is satisfied. Then an upper bound is obtained by

$$
\begin{aligned}
& \left\|\int_{t}^{T}\left|f^{\varphi}\left(s, Y_{s}^{\psi}, Z_{s}^{\psi}\right)-f^{\psi}\left(s, Y_{s}^{\psi}, Z_{s}^{\psi}\right)\right| d s\right\|_{p} \\
\leq & \left\|\int_{t}^{T}\left|Z_{s}^{\psi}\right|^{1+\theta}\left|A_{s}^{\varphi}-A_{s}^{\psi}\right| d s\right\|_{p} \\
\leq & \left\|\left(\int_{t}^{T}\left|Z_{s}^{\psi}\right|^{2} d s\right)^{\frac{1+\theta}{2}}\left(\int_{t}^{T}\left|A_{s}^{\varphi}-A_{s}^{\psi}\right|^{\frac{2}{1-\theta}} d s\right)^{\frac{1-\theta}{2}}\right\|_{p} \\
\leq & \left\|\left(\int_{t}^{T}\left|Z_{s}\right|^{2} d s\right)^{\frac{1}{2}}\right\|_{(1+\theta) p_{0}}^{1+\theta}\left\|\left(\int_{t}^{T}\left|A_{s}^{\varphi}-A_{s}^{\psi}\right|^{\frac{2}{1-\theta}} d s\right)^{\frac{1-\theta}{2}}\right\|_{p_{1}}
\end{aligned}
$$

for any $\frac{1}{p}=\frac{1}{p_{0}}+\frac{1}{p_{1}}$ with $p<p_{0}, p_{1}<\infty$, where we use Remark 2.14(2) in the last step. 
6.4.3. Theorem 6.3 for the perturbation $(\varphi, \psi)=\left(\chi_{(a, b]}, 0\right)$. The importance of the pair $(\varphi, \psi)=\left(\chi_{(a, b]}, 0\right)$ follows from the fact that

$$
\left\|Y_{t}-Y_{t}^{(t-\varepsilon, t]}\right\|_{p} \sim_{2}\left\|Y_{t}-\mathbb{E}\left(Y_{t} \mid \mathcal{F}_{t-\varepsilon}\right)\right\|_{p}
$$

for $p \in[1, \infty]$, i.e. the fractional smoothness of $Y_{t}$ is measured in terms of the speed of convergence of the conditional expectations. In the case $(\varphi, \psi)=\left(\chi_{(a, b]}, 0\right)$ we have that (38) implies two inequalities that give different information about the $L_{p^{-}}$ variation of the processes $Y=\left(Y_{t}\right)_{t \in[0, T]}$ and $Z=\left(Z_{t}\right)_{t \in[0, T]}$ : Firstly, for $0<\varepsilon<t$ we have that

$$
\begin{aligned}
& \left\|Y_{t}-Y_{t}^{(t-\varepsilon, t]}\right\|_{p}+\left\|\left(\int_{t}^{T}\left|Z_{s}-Z_{s}^{(t-\varepsilon, t]}\right|^{2} d s\right)^{\frac{1}{2}}\right\|_{p} \\
& \quad \leq c \sqrt{6.3}\left[\left\|\xi-\xi^{(t-\varepsilon, t]}\right\|_{p}+\left\|\int_{t}^{T}\left|f\left(s, Y_{s}, Z_{s}\right)-f^{(t-\varepsilon, t]}\left(s, Y_{s}, Z_{s}\right)\right| d s\right\|_{p}\right]
\end{aligned}
$$

and, secondly,

$$
\begin{aligned}
& \left\|\left(\int_{t-\varepsilon}^{t}\left|Z_{s}\right|^{2} d s\right)^{\frac{1}{2}}\right\|_{p} \\
& \leq c_{\underline{6.3}}\left[\left\|\xi-\xi^{(t-\varepsilon, t]}\right\|_{p}+\left\|\int_{t-\varepsilon}^{T}\left|f\left(s, Y_{s}, Z_{s}\right)-f^{(t-\varepsilon, t]}\left(s, Y_{s}, Z_{s}\right)\right| d s\right\|_{p}\right] .
\end{aligned}
$$

6.4.4. Theorem 6.3 and Besov spaces. We want to transform Theorem 6.3 into an embedding theorem for the Besov spaces $\mathbb{B}_{p}^{\Phi}$. As the BSDEs we consider might be even quadratic we have - in some sense - a non-linear embedding theorem. To handle the assumption on the generator we need a slight extension of our anisotropic Besov spaces:

Definition 6.19. For $q, r \in[1, \infty)$, a predictable process $\left(A_{t}\right)_{t \in[0, T]}$ with

$$
\left\|\left(\int_{0}^{T}\left|A_{s}\right|^{r} d s\right)^{\frac{1}{r}}\right\|_{q}<\infty,
$$

for $t \in[0, T]$, and for an admissible functional $\Phi$ we let

$$
\|A\|_{\Phi, q}^{r, t}:=\Phi\left(\psi \rightarrow\left\|\left(\int_{t}^{T}\left|A_{s}-A_{s}^{\psi}\right|^{r} d s\right)^{\frac{1}{r}}\right\|_{q}\right) .
$$

First we show that this definition is possible:

Lemma 6.20. The map

$$
\psi \rightarrow\left\|\left(\int_{t}^{T}\left|A_{s}-A_{s}^{\psi}\right|^{r} d s\right)^{\frac{1}{r}}\right\|_{q}
$$

is continuous as a map from $\Delta$ into $[0, \infty)$. 
Proof. We fix an $N \geq 1$ and consider the truncation $A_{t}^{N}:=(-N) \vee\left(A_{t} \wedge N\right)$. For $u:=q \vee r$ and $\psi_{n}, \psi \in \Delta$ we get

$$
\begin{aligned}
& \left\|\left(\int_{t}^{T}\left|A_{s}^{\psi_{n}}-A_{s}^{\psi}\right|^{r} d s\right)^{\frac{1}{r}}\right\|_{q} \\
\leq & \left\|\left(\int_{t}^{T}\left|A_{s}^{\psi_{n}}-\left(A^{N}\right)_{s}^{\psi_{n}}\right|^{r} d s\right)^{\frac{1}{r}}\right\|_{q}+\left\|\left(\int_{t}^{T}\left|\left(A^{N}\right)_{s}^{\psi_{n}}-\left(A^{N}\right)_{s}^{\psi}\right|^{r} d s\right)^{\frac{1}{r}}\right\|_{q} \\
& +\left\|\left(\int_{t}^{T}\left|\left(A^{N}\right)_{s}^{\psi}-A_{s}^{\psi}\right|^{r} d s\right)^{\frac{1}{r}}\right\|_{q} \\
= & 2\left\|\left(\int_{t}^{T}\left|A_{s}-\left(A^{N}\right)_{s}\right|^{r} d s\right)^{\frac{1}{r}}\right\|_{q}+\left\|\left(\int_{t}^{T}\left|\left(A^{N}\right)_{s}^{\psi_{n}}-\left(A^{N}\right)_{s}^{\psi}\right|^{r} d s\right)^{\frac{1}{r}}\right\|_{q} \\
\leq & 2\left\|\left(\int_{t}^{T}\left|A_{s}-\left(A^{N}\right)_{s}\right|^{r} d s\right)^{\frac{1}{r}}\right\|_{q}+c_{q, r, T}\left(\int_{t}^{T}\left\|\left(A^{N}\right)_{s}^{\psi_{n}}-\left(A^{N}\right)_{s}^{\psi}\right\|_{u}^{u} d s\right)^{\frac{1}{u}}
\end{aligned}
$$

where we used for the equality Remark 2.14(2). Applying dominated convergence twice we get that

$$
\lim _{N}\left\|\left(\int_{t}^{T}\left|A_{s}-\left(A^{N}\right)_{s}\right|^{r} d s\right)^{\frac{1}{r}}\right\|_{q}=0
$$

Moreover, using Proposition 2.5(7) we find a Borel set $B \subseteq[0, T]$ of Lebesgue measure $T$ such that $A_{t}^{\rho}$ is the transformation of $A_{t}$ for any $t \in B$ and $\rho \in\left\{\psi, \psi_{1}, \psi_{2}, \ldots\right\}$. In case $\psi_{n} \rightarrow \psi$ we can therefore apply Lemma 4.7 to conclude the proof because this implies that

$$
\lim _{n}\left(\int_{t}^{T}\left\|\left(A^{N}\right)_{s}^{\psi_{n}}-\left(A^{N}\right)_{s}^{\psi}\right\|_{u}^{u} d s\right)^{\frac{1}{u}}=0
$$

Now we obtain the following embedding theorem:

Corollary 6.21. Assume that the assumptions of Theorem 6.3 are satisfied, $t \in$ $[0, T]$, and that there are predictable processes $\left(V_{s}^{l}\right)_{s \in[t, T]}$ such that, for all $\psi \in \Delta$,

$$
\left\|\int_{t}^{T}\left|f\left(s, Y_{s}^{\psi}, Z_{s}^{\psi}\right)-f^{\psi}\left(s, Y_{s}^{\psi}, Z_{s}^{\psi}\right)\right| d s\right\|_{p} \leq \sum_{l=1}^{L}\left\|V_{.}^{l}-\left(V_{.}^{l}\right)^{\psi}\right\|_{L_{q_{l}}\left(L_{r_{l}}([t, T])\right)}
$$

for some $q_{l} \in[p, \infty)$ and $r_{l} \in[1, \infty){ }^{1}$. Let $\Phi: C^{+}(\Delta) \rightarrow[0, \infty]$ be admissible in the sense of Definition 4.10. Then we have that

$$
\left\|Y_{t}\right\|_{\Phi, p}+\|Z\|_{\Phi, p}^{2, t} \leq 2 c \sqrt{6.3}\left[\|\xi\|_{\Phi, p}+\sum_{l=1}^{L}\left\|V^{l}\right\|_{\Phi, q_{l}}^{r_{l}, t}\right] .
$$

\footnotetext{
${ }^{1}$ The $V^{l}$ may depend on $\left(\xi, f, Y, Z, p, q_{l}, r_{l}\right)$.
} 
Proof. The statement follows directly from Theorem 6.3 applied to the pair $(0, \psi)$.

Examples, how to obtain processes $\left(V_{s}^{l}\right)_{s \in[t, T]}$, can be found in Section 6.4.2. For the sake of illustration we first combine Corollary 6.21 with Theorem 6.13 (note that we use conditions (B3) and (B8)) so that the assumptions of Theorem 6.3 are automatically satisfied with $p=2$ and $s_{\infty}=0$ :

Corollary 6.22. Assume that $\theta \in(0,1), t \in[0, T], \xi \in \mathrm{cExp}$, and that $(Y, Z)$ is the unique solution to the BSDE (34) obtained in Theorem 6.13. Suppose a predictable process $\left(V_{s}\right)_{s \in[t, T]}$ such that

$$
\left\|\int_{t}^{T} \sup _{y, z}\left|f(s, y, z)-f^{\psi}(s, y, z)\right| d s\right\|_{2} \leq\left\|V .-(V .)^{\psi}\right\|_{L_{2}\left(L_{1}([t, T])\right)}
$$

for all $\psi \in \Delta$. Let $\Phi: C^{+}(\Delta) \rightarrow[0, \infty]$ be admissible in the sense of Definition 4.10. Then we have that

$$
\left\|Y_{t}\right\|_{\Phi, 2}+\|Z\|_{\Phi, 2}^{2, t} \leq 2 c_{[6.3]}\left[\|\xi\|_{\Phi, 2}+\|V\|_{\Phi, 2}^{1, t}\right] .
$$

Taking also Theorem 4.22 into the account we obtain another version of Corollary 6.22 that only uses that $\xi$ is locally in $\mathbb{D}_{1,2}$ in the sense to check perturbations of the Gaussian structure up to time $t$ only. This confirms the smoothing effect of a BSDE as this already implies the smoothness of $Y_{t}$. More precisely we get:

Corollary 6.23. Assume that $\theta \in(0,1), t \in[0, T], \xi \in \mathrm{cExp}$, and that $(Y, Z)$ is the unique solution to the BSDE (34) obtained in Theorem 6.13. Then we have

$$
\begin{aligned}
& \operatorname{esssup}_{s \in[0, t]}\left\|D_{s} Y_{t}\right\|_{2} \\
& \leq c \sup _{0 \leq a<b \leq t} \frac{1}{\sqrt{b-a}}\left[\left\|\xi-\xi^{(a, b]}\right\|_{2}+\left\|\int_{t}^{T} \sup _{y, z}\left|f(s, y, z)-f^{(a, b]}(s, y, z)\right| d s\right\|_{2}\right]
\end{aligned}
$$

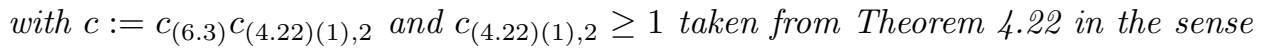
that if the right-hand side is finite, then $Y_{t} \in \mathbb{D}_{1,2}$ and $(52)$ holds.

Proof. We apply Theorems 6.3 and 4.22 , where for the latter we use

$$
Y_{t}^{(a, b]}=Y_{t}^{(a \wedge t, b \wedge t]} \text { a.s. }
$$

because $Y_{t}$ is $\mathcal{F}_{t}$-measurable.

If $\xi \in \mathbb{D}_{1,2}$, then we have that

$$
\sup _{0 \leq a<b \leq t} \frac{\left\|\xi-\xi^{(a, b]}\right\|_{2}}{\sqrt{b-a}} \leq 2 c{ }_{A .7]} \operatorname{esssup}_{s \in[0, t]}\left\|D_{s} \xi\right\|_{2}
$$

by Corollary 6.29, but for Corollary 6.23 the assumption $\xi \in \mathbb{D}_{1,2}$ is not necessary. 


\subsection{On the $L_{p}$-variation of BSDEs}

In this section we show how Theorem 6.3 can be applied in order to obtain information about the $L_{p}$-variation of our BSDE. The link between the $L_{p}$-variation of the $Y$-process and the stability result Theorem 6.3 consists in the observation

$$
\left\|A_{t}-A_{s}\right\|_{p} \leq\left\|A_{t}-\mathbb{E}\left(A_{t} \mid \mathcal{F}_{s}\right)\right\|_{p}+\left\|\mathbb{E}\left(A_{t} \mid \mathcal{F}_{s}\right)-A_{s}\right\|_{p} \leq 3\left\|A_{t}-A_{s}\right\|_{p},
$$

where $p \in[1, \infty],\left(A_{t}\right)_{t \in[0, T]} \subseteq \mathcal{L}_{p}$ is adapted, and $0 \leq s \leq t \leq T$. Our estimate for the $Z$-process will follow directly from Theorem 6.3 .

In Remark 6.33(1) below we show that under the conditions $\int_{0}^{T}\left\|Z_{r}\right\|_{p}^{2} d r<\infty$ and $\int_{0}^{T}\left\|f\left(r, Y_{r}, Z_{r}\right)\right\|_{p} d r<\infty$, and under the a-priori knowledge of the behaviour of the functions $r \rightarrow\left\|Z_{r}\right\|_{p}$ and $r \rightarrow\left\|f\left(r, Y_{r}, Z_{r}\right)\right\|_{p}$ one gets a rate of $1 / \sqrt{n}$ for the $L_{p}$-variation of $Y$ and $Z$ by adapted time-nets. In Corollary 6.32 below we will deduce estimates with explicit adapted time-nets where we only assume conditions on the initial data $(\xi, f)$. Regarding the case $p \in(2, \infty)$ there is another aspect: In Remark 6.33(2) we show that even for the zero generator case one might have situations where one cannot achieve the rate $1 / \sqrt{n}$ for the variation of $Y$, i.e. the variation of $Y$ is asymptotically higher. Our sufficient conditions give cases where one gets the rate $1 / \sqrt{n}$ for the case $p \in(2, \infty)$.

In the following the random variables are considered on the product space $\bar{\Omega}$ if necessary. In particular, random variables defined on $\Omega$ are automatically extended to $\bar{\Omega}$ in the natural way when needed.

TheOREM 6.24. Suppose that the assumptions of Theorem 6.3 are satisfied. Then, for $\left.c_{\sqrt{6.24})}:=c_{\sqrt{6.3}\left[1+c_{\sqrt{5.19}, p} L_{Z}\right.}(\sqrt{T}+1)\right]$ and $0 \leq s<t \leq T$, one has

$$
\begin{aligned}
& \left\|\sup _{r \in[s, t]}\left|Y_{r}-Y_{s}\right|\right\|_{p}+\left\|\left(\int_{s}^{t}\left|Z_{r}\right|^{2} d r\right)^{\frac{1}{2}}\right\|_{p} \\
\leq & \left\|\int_{s}^{t}|f(r, 0,0)| d r\right\|_{p}+L_{Y}(t-s) \sup _{r \in[0, T]}\left\|Y_{r}\right\|_{p}+c_{[6.24}\left[1+\left\|\left.Z\right|^{\theta}\right\|_{\operatorname{BMO}\left(S_{2}\right)}\right] \times \\
& \times\left[\left\|\xi-\xi^{(s, t]}\right\|_{p}+\left\|\int_{s}^{T}\left|f\left(r, Y_{r}, Z_{r}\right)-f^{(s, t]}\left(r, Y_{r}, Z_{r}\right)\right| d r\right\|_{p}\right] .
\end{aligned}
$$

Proof. We fix $0 \leq s<t \leq T$ and remark that $\left\|\int_{s}^{t}|f(r, 0,0)| d r\right\|_{p}<\infty$ according to condition (B5). We let $\left(q_{l}\right)_{l=1}^{\infty}$ be an enumeration of the rational numbers from $(s, t]$ so that

$$
\left\|\sup _{r \in[s, t]}\left|Y_{r}-Y_{s}\right|\right\|_{p}=\sup _{m=1,2, \ldots}\left\|\sup _{q \in\left\{q_{1}, \ldots, q_{m}\right\}}\left|Y_{q}-Y_{s}\right|\right\|_{p}
$$

by monotone convergence. Using Lemma 4.23 , the fact that the $Y_{q_{l}}$ are $\mathcal{F}_{t}$-measurable, and Theorem 6.3 we obtain that 


$$
\begin{aligned}
& \left\|\sup _{q \in\left\{q_{1}, \ldots, q_{m}\right\}}\left|Y_{q}-Y_{s}\right|\right\|_{p}+\left\|\left(\int_{s}^{t}\left|Z_{r}\right|^{2} d r\right)^{\frac{1}{2}}\right\|_{p} \\
& \leq\left\|\sup _{q \in\left\{q_{1}, \ldots, q_{m}\right\}}\left|Y_{q}-\mathbb{E}^{\mathcal{F}_{s}} Y_{q}\right|\right\|_{p}+\left\|\sup _{q \in\left\{q_{1}, \ldots, q_{m}\right\}}\left|\mathbb{E}^{\mathcal{F}_{s}} Y_{q}-Y_{s}\right|\right\|_{p} \\
& +\left\|\left(\int_{s}^{t}\left|Z_{r}\right|^{2} d r\right)^{\frac{1}{2}}\right\|_{p} \\
& \leq\left\|\sup _{q \in\left\{q_{1}, \ldots, q_{m}\right\}}\left|Y_{q}-Y_{q}^{(s, t]}\right|\right\|_{p}+\left\|\left(\int_{s}^{t}\left|Z_{r}\right|^{2} d r\right)^{\frac{1}{2}}\right\|_{p} \\
& +\left\|\sup _{q \in\left\{q_{1}, \ldots, q_{m}\right\}}\left|\mathbb{E}^{\mathcal{F}_{s}} Y_{q}-Y_{s}\right|\right\|_{p} \\
& \leq \quad c \underline{6.3}\left[\left\|\xi-\xi^{(s, t]}\right\|_{p}+\left\|\int_{s}^{T}\left|f\left(r, Y_{r}, Z_{r}\right)-f^{(s, t]}\left(r, Y_{r}, Z_{r}\right)\right| d r\right\|_{p}\right] \\
& +\left\|\sup _{q \in\left\{q_{1}, \ldots, q_{m}\right\}}\left|\mathbb{E}^{\mathcal{F}_{s}} Y_{q}-Y_{s}\right|\right\|_{p} .
\end{aligned}
$$

By Corollary 5.19 and 51 we bound the last term by

$$
\begin{aligned}
& \left\|\sup _{q \in\left\{q_{1}, \ldots, q_{m}\right\}}\left|\mathbb{E}^{\mathcal{F}_{s}} Y_{q}-Y_{s}\right|\right\|_{p} \\
& =\left\|\sup _{q \in\left\{q_{1}, \ldots, q_{m}\right\}}\left|\mathbb{E}^{\mathcal{F}_{s}} \int_{s}^{q} f\left(r, Y_{r}, Z_{r}\right) d r\right|\right\|_{p} \\
& \leq\left\|\int_{s}^{t}\left|f\left(r, Y_{r}, Z_{r}\right)\right| d r\right\|_{p} \\
& \leq\left\|\int_{s}^{t}|f(r, 0,0)| d r\right\|_{p}+L_{Y}\left\|\int_{s}^{t}\left|Y_{r}\right| d r\right\|_{p}+L_{Z}\left\|\int_{s}^{t}\left[1+\left|Z_{r}\right|\right]^{\theta}\left|Z_{r}\right| d r\right\|_{p} \\
& \leq\left\|\int_{s}^{t}|f(r, 0,0)| d r\right\|_{p}+L_{Y}(t-s) \sup _{r \in[s, t]}\left\|Y_{r}\right\|_{p} \\
& +L_{Z} c \underline{5.19], p}\left\|\left(\int_{s}^{t}\left|Z_{r}\right|^{2} d r\right)^{\frac{1}{2}}\right\|_{p}\left\|\left(\chi_{(s, t]}(r)\left[1+\left|Z_{r}\right|\right]^{\theta}\right)_{r \in[0, T]}\right\|_{\operatorname{BMO}\left(S_{2}\right)} \\
& \leq\left\|\int_{s}^{t}|f(r, 0,0)| d r\right\|_{p}+L_{Y}(t-s) \sup _{r \in[s, t]}\left\|Y_{r}\right\|_{p} \\
& +L_{Z} c \sqrt{5.195}, p c[6.3]\left[\left\|\xi^{(s, t]}\right\|_{p}+\left\|\int_{s}^{T}\left|f\left(r, Y_{r}, Z_{r}\right)-f^{(s, t]}\left(r, Y_{r}, Z_{r}\right)\right| d r\right\|_{p}\right] \\
& \times\left[\sqrt{t-s}+|||Z|^{\theta} \|_{\operatorname{BMO}\left(S_{2}\right)}\right] .
\end{aligned}
$$


As remarked in the beginning of the proof of Theorem 6.3 we have $\sup _{r \in[0, T]}\left|Y_{r}\right| \in$ $\mathcal{L}_{p}$ so that $\alpha_{p}:=L_{Y} \sup _{r \in[0, T]}\left\|Y_{r}\right\|_{p}<\infty$. Therefore,

$$
\begin{aligned}
\left\|\sup _{q \in\left\{q_{1}, \ldots, q_{m}\right\}}\left|\mathbb{E}^{\mathcal{F}_{s}} Y_{q}-Y_{s}\right|\right\|_{p} \leq\left\|\int_{s}^{t}|f(r, 0,0)| d r\right\|_{p}+\alpha_{p}(t-s) \\
+\beta_{p}\left[\left\|\xi-\xi^{(s, t]}\right\|_{p}+\left\|\int_{s}^{T}\left|f\left(r, Y_{r}, Z_{r}\right)-f^{(s, t]}\left(r, Y_{r}, Z_{r}\right)\right| d r\right\|_{p}\right]
\end{aligned}
$$

for $\left.\beta_{p}:=L_{Z} c \sqrt[5.19,, p]{ } c \sqrt[6.3]\right]{6}\left[\sqrt{T}+\left\||Z|^{\theta}\right\|_{\mathrm{BMO}\left(S_{2}\right)}\right]$.

The variation of our BSDE we measure by the following quantity:

Definition 6.25. Let $p \in[1, \infty), A=\left(A_{t}\right)_{t \in[0, T]}$ be a measurable càdlàg process $A:[0, T] \times \Omega \rightarrow \mathbb{R}$, and $C=\left(C_{t}\right)_{t \in[0, T]}$ be a measurable process $C:[0, T] \times \Omega \rightarrow \mathbb{R}^{d}$. For a deterministic time-net $\tau=\left(t_{i}\right)_{i=0}^{n}$ with $0=t_{0} \leq t_{1} \leq \cdots \leq t_{n}=T$ we let

$$
\operatorname{var}_{p}([A, C] \mid \tau):=\sup _{i=1, \ldots, n}\left\|\sup _{t_{i-1} \leq s \leq t \leq t_{i}}\left|A_{t}-A_{s}\right|\right\|_{p}+\sup _{i=1, \ldots, n}\left\|\left(\int_{t_{i-1}}^{t_{i}}\left|C_{r}\right|^{2} d r\right)^{\frac{1}{2}}\right\|_{p} .
$$

The variation $\operatorname{var}_{p}([A, C] \mid \tau)$ behaves sub-additive as expected:

Lemma 6.26. For $p \in[1, \infty)$, families $\left(\left(A_{t}^{j}, C_{t}^{j}\right)\right)_{t \in[0, T]}$ and time-nets $\tau^{j}, j=0,1$, as in Definition 6.25, one has that

$$
\operatorname{var}_{p}\left(\left[A^{0}+A^{1}, C^{0}+C^{1}\right] \mid \tau^{0} \cup \tau^{1}\right) \leq \operatorname{var}_{p}\left(\left[A^{0}, C^{0}\right] \mid \tau^{0}\right)+\operatorname{var}_{p}\left(\left[A^{1}, C^{1}\right] \mid \tau^{1}\right) .
$$

Proof. Assume that $\tau=\left(t_{i}\right)_{i=0}^{n_{0}+n_{1}-1}$ is an ordering of the union of $\tau^{0}=$ $\left(t_{i}^{0}\right)_{i=0}^{n_{0}}$ and $\tau^{1}=\left(t_{i}^{1}\right)_{i=0}^{n_{1}}$. Then one has that the interval $\left[t_{i-1}, t_{i}\right]$ is contained in a closed interval of $\tau^{0}$ and, at the same time, in a closed interval of $\tau^{1}$, so that

$$
\begin{aligned}
&\left\|\sup _{t_{i-1} \leq s \leq t \leq t_{i}}\left|\left(A_{t}^{0}+A_{t}^{1}\right)-\left(A_{s}^{0}+A_{s}^{1}\right)\right|\right\|_{p}+\left\|\left(\int_{t_{i-1}}^{t_{i}}\left|C_{r}^{0}+C_{r}^{1}\right|^{2} d r\right)^{\frac{1}{2}}\right\|_{p} \\
& \leq\left\|\sup _{t_{i-1} \leq s \leq t \leq t_{i}}\left|A_{t}^{0}-A_{s}^{0}\right|\right\|_{p}+\left\|\left(\int_{t_{i-1}}^{t_{i}}\left|C_{r}^{0}\right|^{2} d r\right)^{\frac{1}{2}}\right\|_{p} \\
&+\left\|\sup _{t_{i-1} \leq s \leq t \leq t_{i}}\left|A_{t}^{1}-A_{s}^{1}\right|\right\|_{p}+\left\|\left(\int_{t_{i-1}}^{t_{i}}\left|C_{r}^{1}\right|^{2} d r\right)^{\frac{1}{2}}\right\|_{p} \\
& \leq \operatorname{var}_{p}\left(\left[A^{0}, C^{0}\right] \mid \tau^{0}\right)+\operatorname{var}_{p}\left(\left[A^{1}, C^{1}\right] \mid \tau^{1}\right) .
\end{aligned}
$$

Now we formulate consequences of Theorem 6.24 in two different scenarios: The first Corollary 6.31 still relies on the assumptions of Theorem 6.3. In the next step Corollary 6.31 will be combined with the results from Section 6.3 to guarantee the validity of the assumptions of Theorem 6.3. This yields to Corollary 6.32.

To shorten the formulation of the statements we work with the following two definitions. 
The first definition extends the spaces $\mathbb{B}_{p}^{\Phi}$ to the initial data $(\xi, f)$ of the BSDE:

Definition 6.27. We say that $(\xi, f) \in \mathbb{B}_{p}^{\Phi_{\gamma, \Gamma}}$, where $p \in(0, \infty), \gamma \in[2, \infty)$, and $\Gamma:[0, T] \rightarrow[0, \infty)$ is integrable, provided that $\xi \in L_{p}$ and for all $0 \leq a<b \leq T$,

$$
\left\|\xi-\xi^{(a, b]}\right\|_{p}+\left\|\int_{a}^{T} \sup _{(y, z) \in \mathbb{R}^{d+1}}\left|f(r, y, z)-f^{(a, b]}(r, y, z)\right| d r\right\|_{p} \leq\left(\int_{a}^{b} \Gamma(r) d r\right)^{\frac{1}{\gamma}} .
$$

The term $\int_{a}^{T} \sup _{(y, z) \in \mathbb{R}^{d+1}}\left|f(r, y, z)-f^{(a, b]}(r, y, z)\right| d r$ is an extended random variable on $(\bar{\Omega}, \overline{\mathcal{F}}, \overline{\mathbb{P}})$. Concerning the generator, the above definition reflects the situation described in Example 6.16, where the generator $f$ is obtained from some appropriate $h$ with

$$
f(r, \omega, y, z):=h\left(r, A_{r}(\omega), y, z\right) .
$$

The second definition recalls a well-known principle to generate adapted time-nets:

Definition 6.28. Letting $\Lambda:[0, T] \rightarrow(0, \infty)$ be integrable and $n \geq 1$, the time-net $\tau_{n}^{\Lambda}$ consists of $0=t_{0}<\cdots<t_{n}=T$ such that, for all $i=1, \ldots, n$,

$$
\int_{t_{i-1}}^{t_{i}} \Lambda(r) d r=\frac{1}{n} \int_{0}^{T} \Lambda(r) d r
$$

The following corollary, which follows directly from Lemma 4.23 and 24 , yields to the fundamental example for $\gamma=2$ concerning the part $\left\|\xi-\xi^{(a, b]}\right\|_{p}$ in Definition 6.27 above:

Corollary 6.29. For $p \in[2, \infty)$ and $\xi \in \mathbb{D}_{1,2} \cap L_{p}$ with $\int_{(0, T]}\left\|D_{r} \xi\right\|_{p}^{2} d r<\infty$ one has for all $0 \leq a<b \leq T$ that

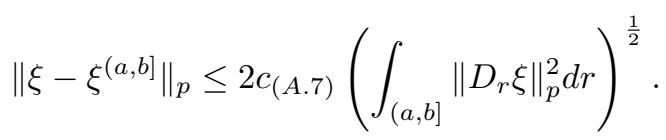

Remark 6.30. For $\Gamma>0$ Definition 6.27 and Example 4.11 yield to the admissible functional

$$
\Phi_{\gamma, \Gamma}(F):=\sup _{0 \leq a<b \leq T} \frac{F\left(\chi_{(a, b]}\right)}{\sqrt[\gamma]{\int_{a}^{b} \Gamma(r) d r}}
$$

that recovers the functional $\Phi_{\gamma}$ from $(25)$ by $\Gamma \equiv 1$.

Our first corollary of Theorem 6.24 is

Corollary 6.31. Let $p, \gamma \in[2, \infty)$ and $\Gamma:[0, T] \rightarrow[0, \infty)$ be integrable. Suppose the assumptions of Theorem 6.3. $(\xi, f) \in \mathbb{B}_{p}^{\Phi_{\gamma, \Gamma}}$ and $\int_{0}^{T}\|f(r, 0,0)\|_{p} d r<\infty$. Then

$$
\operatorname{var}_{p}\left([Y, Z] \mid \tau_{n}^{\Lambda}\right) \leq \frac{c \sqrt{6.31]}}{n}+\frac{d \sqrt{6.31]}}{\sqrt[r]{n}}
$$

for $\Lambda(r):=1+\|f(r, 0,0)\|_{p}+\Gamma(r)$ and

$$
\begin{aligned}
& c_{[6.31]}:=2\|\Lambda\|_{L_{1}([0, T])}\left[1+L_{Y} \sup _{t \in[0, T]}\left\|Y_{t}\right\|_{p}\right], \\
& d \sqrt{6.31]}:=2 c_{[6.24]}\|\Lambda\|_{L_{1}([0, T])}^{\frac{1}{\gamma}}\left[1+\left\||Z|^{\theta}\right\|_{\mathrm{BMO}\left(S_{2}\right)}\right] .
\end{aligned}
$$


Proof. For $0 \leq s<t \leq T$ Theorem 6.24 implies that

$$
\begin{aligned}
& \left\|\sup _{r \in[s, t]}\left|Y_{r}-Y_{s}\right|\right\|_{p}+\left\|\left(\int_{s}^{t}\left|Z_{r}\right|^{2} d r\right)^{\frac{1}{2}}\right\|_{p} \\
& \leq\left\|\int_{s}^{t}|f(r, 0,0)| d r\right\|_{p}+L_{Y}(t-s) \sup _{r \in[0, T]}\left\|Y_{r}\right\|_{p}
\end{aligned}
$$

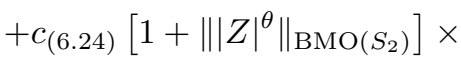

$$
\begin{aligned}
& \times\left[\left\|\xi-\xi^{(s, t]}\right\|_{p}+\left\|\int_{s}^{T}\left|f\left(r, Y_{r}, Z_{r}\right)-f^{(s, t]}\left(r, Y_{r}, Z_{r}\right)\right| d r\right\|_{p}\right] \\
& \leq \int_{s}^{t}\|f(r, 0,0)\|_{p} d r+L_{Y}(t-s) \sup _{r \in[0, T]}\left\|Y_{r}\right\|_{p} \\
& +c \sqrt{6.24}\left[1+\left\||Z|^{\theta}\right\|_{\mathrm{BMO}\left(S_{2}\right)}\right]\left(\int_{s}^{t} \Gamma(r) d r\right)^{\frac{1}{\gamma}} .
\end{aligned}
$$

Assuming $0=t_{0} \leq t_{1} \leq \cdots \leq t_{n}=T$ we conclude by

$$
\begin{aligned}
& \left\|\sup _{t_{t-1} \leq s \leq t \leq t_{i}}\left|Y_{t}-Y_{s}\right|\right\|_{p}+\left\|\left(\int_{t_{i-1}}^{t_{i}}\left|Z_{r}\right|^{2} d r\right)^{\frac{1}{2}}\right\|_{p} \\
& \leq 2\left\|\sup _{r \in\left[t_{t-1}, t_{i}\right]}\left|Y_{r}-Y_{t_{i-1}}\right|\right\|_{p}+\left\|\left(\int_{t_{i-1}}^{t_{i}}\left|Z_{r}\right|^{2} d r\right)^{\frac{1}{2}}\right\|_{p} \\
& \leq 2\left[\int_{t_{i-1}}^{t_{i}}\|f(r, 0,0)\|_{p} d r+L_{Y}\left(t_{i}-t_{i-1}\right) \sup _{r \in[0, T]}\left\|Y_{r}\right\|_{p}\right.
\end{aligned}
$$

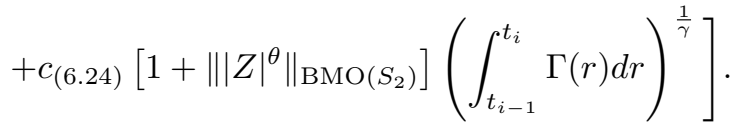

Corollary 6.32. Assume $\gamma \in[2, \infty)$, an integrable $\Gamma:[0, T] \rightarrow[0, \infty)$, and that one of the following sets of conditions is satisfied:

(1) $\theta=0, p \in[2, \infty), \xi \in L_{p},(B 3), \int_{0}^{T}\|f(r, 0,0)\|_{p} d r<\infty,(\xi, f) \in \mathbb{B}_{p}^{\Phi_{\gamma, \Gamma}}$.

(2) $\theta \in(0,1), \xi \in \mathrm{cExp},(B 3),(B 8)$, and $(\xi, f) \in \mathbb{B}_{2}^{\Phi_{\gamma, \Gamma}}$.

(3) $\theta=1, \xi \in L_{\infty}$, (B3), (B8), and $(\xi, f) \in \bigcap_{q \in[2, \infty)} \mathbb{B}_{q}^{\Phi_{\gamma, \Gamma}}$.

Define the weight function

$$
\Lambda(r):=1+\|f(r, 0,0)\|_{u}+\Gamma(r)
$$

where $u=p$ for $\theta=0, u=2$ for $\theta \in(0,1)$, and $u=\infty$ for $\theta=1$. Then one has that

$$
\sup _{n \geq 1} \sqrt[\gamma]{n} \operatorname{var}_{v}\left([Y, Z] \mid \tau_{n}^{\Lambda}\right)<\infty
$$

for $v=p$ if $\theta=0, v=2$ if $\theta \in(0,1)$, and for all $v \in(0, \infty)$ if $\theta=1$, where for $\theta=0$ the solution is taken from [21. Theorem 4.2], for $\theta \in(0,1)$ from Theorem 6.13 , and for $\theta=1$ from Remark 6.14 . 
Proof. The statement follows by a combination of Table 1 (cases (I), (II), and (III)) and Corollary 6.31. For part (3) we remark that we first deduce our statement for $v \in[2, \infty) \cap\left(p_{0}, \infty\right)$ with $p_{0}$ taken from Theorem 6.3, and then (obviously) the conclusion follows for all $v \in(0, \infty)$.

REMARK 6.33 .

(1) For $p \in[2, \infty)$ assume for our BSDE the conditions $\int_{0}^{T}\left\|Z_{r}\right\|_{p}^{2} d r<\infty$ and $\int_{0}^{T}\left\|f\left(r, Y_{r}, Z_{r}\right)\right\|_{p} d r<\infty$. Take a net $\tau^{n}=\left(t_{i}^{n}\right)_{i=1}^{n}$ that satisfies

$$
\int_{t_{i-1}^{n}}^{t_{i}^{n}}\left[\left\|f\left(r, Y_{r}, Z_{r}\right)\right\|_{p}+\left\|Z_{r}\right\|_{p}^{2}\right] d r=\frac{1}{n} \int_{0}^{T}\left[\left\|f\left(r, Y_{r}, Z_{r}\right)\right\|_{p}+\left\|Z_{r}\right\|_{p}^{2}\right] d r .
$$

Given $i \in\{1, \ldots, n\}$ we derive

$$
\begin{aligned}
&\left\|\sup _{t_{i-1}^{n} \leq s \leq t \leq t_{i}^{n}}\left|Y_{t}-Y_{s}\right|\right\|_{p}+\left\|\left(\int_{t_{i-1}^{n}}^{t_{i}^{n}}\left|Z_{r}\right|^{2} d r\right)^{\frac{1}{2}}\right\| \|_{p} \\
& \leq\left\|\int_{t_{i-1}^{n}}^{t_{i}^{n}}\left|f\left(r, Y_{r}, Z_{r}\right)\right| d r\right\|_{p}+\left\|\sup _{q \in\left[t_{i-1}^{n}, t_{i}^{n}\right]}\left|\int_{t_{i-1}^{n}}^{q} Z_{r} d W_{r}\right|\right\|_{p} \\
&+\left\|\left(\int_{t_{i-1}^{n}}^{t_{i}^{n}}\left|Z_{r}\right|^{2} d r\right)^{\frac{1}{2}}\right\| \|_{p} \\
& \leq \int_{t_{i-1}}^{t_{i}}\left\|f\left(r, Y_{r}, Z_{r}\right)\right\|_{p} d r+\left[2 \beta_{p}+1\right]\left\|\left(\int_{t_{i-1}}^{t_{i}}\left|Z_{r}\right|^{2} d r\right)^{\frac{1}{2}}\right\|_{p} \\
& \leq \int_{t_{i-1}}^{t_{i}}\left\|f\left(r, Y_{r}, Z_{r}\right)\right\|_{p} d r+\left[2 \beta_{p}+1\right]\left(\int_{t_{i-1}}^{t_{i}}\left\|Z_{r}\right\|_{p}^{2} d r\right)^{\frac{1}{2}} \\
& \leq \frac{1}{n} \int_{0}^{T}\left[\left\|f\left(r, Y_{r}, Z_{r}\right)\right\|_{p}+\left\|Z_{r}\right\|_{p}^{2}\right] d r \\
&+\frac{2 \beta_{p}+1}{\sqrt{n}}\left(\int_{0}^{T}\left[\left\|f\left(r, Y_{r}, Z_{r}\right)\right\|_{p}+\left\|Z_{r}\right\|_{p}^{2}\right] d r\right)^{\frac{1}{2}}
\end{aligned}
$$

where the Burkholder-Davis-Gundy inequalities (11) were exploited. Consequently, we have a variation of $1 / \sqrt{n}$ by taking the nets $\tau^{n}$.

(2) However, in general for $p \in(2, \infty)$ such an estimate is not always possible as shown by the following example for $d=1$ : Take an infinite time-net converging to $T$,

$$
0=t_{0}<t_{1}<t_{2}<\cdots,
$$

and pair-wise disjoint $A_{k} \in \mathcal{F}_{t_{k}}$ of positive measure for $k=1,2, \ldots$ (Given pair-wise disjoint non-empty finite intervals $I_{k}=\left(a_{k}, b_{k}\right)$ one can choose $A_{1}:=\left\{W_{t_{1}} \in I_{1}\right\}$ and $A_{k}:=\left\{W_{t_{1}} \notin I_{1}, \ldots, W_{t_{k-1}} \notin I_{k-1}, W_{t_{k}} \in I_{k}\right\}$ for $k \geq 2$.) For $\left(\alpha_{k}\right)_{k=1}^{\infty} \subset(0, \infty)$ and $s \in[0, T]$ define

$$
\lambda_{s}:=\sum_{k=2}^{\infty} \alpha_{k-1} \chi_{A_{k-1}} \chi_{\left(t_{k-1}, t_{k}\right]}(s) .
$$


Let $0<\alpha<\frac{p}{2}-1$ and arrange the $\alpha_{k}$ such that

$$
\left\|\int_{\left(t_{k-1}, t_{k}\right]} \lambda_{s} d W_{s}\right\|_{p}=k^{-\frac{1+\alpha}{p}}
$$

which implies

$$
\mathbb{E}\left|\int_{(0, T]} \lambda_{s} d W_{s}\right|^{p}=\sum_{k=2}^{\infty} k^{-(1+\alpha)}<\infty .
$$

Let us assume $c>0$ and a sequence of time-nets $\tau^{n}, 0=t_{0}^{n} \leq \cdots \leq t_{n}^{n}=$ $T$, such that

$$
\left\|Y_{t_{i}^{n}}-Y_{t_{i-1}^{n}}\right\|_{p} \leq \frac{c}{\sqrt{n}} \quad \text { for } \quad Y_{t}:=\int_{0}^{t} \lambda_{s} d W_{s} .
$$

Then $\left(t_{k-1}, t_{k}\right) \cap \tau^{n}=\emptyset$ for $k \geq 2$ implies that

$$
\left\|Y_{t_{k}}-Y_{t_{k-1}}\right\|_{p}=k^{-\frac{1+\alpha}{p}} \leq \frac{c}{\sqrt{n}}
$$

or, equivalently, the condition $k^{-\frac{1+\alpha}{p}}>\frac{c}{\sqrt{n}}$ gives $\left(t_{k-1}, t_{k}\right) \cap \tau^{n} \neq \emptyset$ for $k \geq 2$. In other words, all intervals $\left(t_{k-1}, t_{k}\right)$ with

$$
2 \leq k<\left(\frac{\sqrt{n}}{c}\right)^{\frac{p}{1+\alpha}}
$$

contain at least one element of the time-net $\tau^{n}$. This gives a contradiction to $\frac{p}{2(1+\alpha)}>1$.

\subsection{Applications to other types of BSDEs}

The decoupling techniques developed in this article rely only on the existence of solutions to BSDEs, not on their uniqueness nor on special techniques to prove existence or uniqueness. This opens the possibility to apply the results and techniques to other types of BSDEs as well. Let us list some potential examples:

(1) Multidimensional BSDEs \& COUPLed forward-BACKWARd SDEs

Theorem 3.3 is flexible enough to treat in 13 an $\mathbb{R}^{n}$-valued process $\left(L_{t}\right)_{t \in[0, T]}$ by considering its coordinates separately. This might be applied to BSDEs where the $Y$-process is multi-dimensional. Moreover, if in coupled forward-backward SDEs (see for example 64) the dependencies in the forward diffusion on the backward component can be handled by Theorem 3.3 . then our decoupling approach can be directly examined as well.

(2) BSDEs With Singular terminal CONDitions

Singular terminal conditions are considered for instance in $\mathbf{7 6}, \mathbf{3}$. The general idea behind this type of singular terminal condition for BSDEs consists in replacing the one-parametric family of equations from $t$ to $T$ by the two-parametric family

$$
Y_{t}=Y_{r}+\int_{t}^{r} f\left(s, Y_{s}, Z_{s}\right) d s-\int_{t}^{r} Z_{s} d W_{s} \quad \text { for } \quad 0 \leq t<r<T
$$


and to look for solutions $\left(Y_{t}, Z_{t}\right)_{t \in[0, T)}$ where the process $Y$ is subject to constraints as $t \uparrow T$. Let us indicate how the process $Y$ from (34) might be mapped into $Y^{\rho}$ like in 35 for constraints of the form $\lim _{t \uparrow T} Y_{t}=\infty$ on $\Omega^{+}$or $\lim _{t \uparrow T} Y_{t}=-\infty$ on $\Omega^{-}$for some $\Omega^{ \pm} \in \mathcal{F}_{T}$ of positive measure. With $h:=\arctan :[-\infty, \infty] \rightarrow[-\pi / 2, \pi / 2]$ the transformed process $Y^{h}:=$ $\left(h\left(Y_{t}\right)\right)_{t \in[0, T]}$ is continuous and takes values in $[-\pi / 2, \pi / 2]$. This process $Y^{h}$ can be mapped into $\left(Y^{h}\right)^{\rho}$ as in $\left(35\right.$, and by changing $\left(Y^{h}\right)^{\rho}$ on a set of measure zero we may assume as well that $\left(Y^{h}\right)^{\rho}$ takes values in $[-\pi / 2, \pi / 2]$ only. Applying $h^{-1}$ gives a candidate for $Y^{\rho}$.

(3) Extension to Lévy PRocesses

Our approach in Chapter2 2 is not restricted to particular distributions and its general presentation is intended to apply the results in other settings than the Wiener space as well. A first natural candidate are BSDEs driven by Lévy processes. Here first results were obtained in [41, where a decoupling is used in $L_{2}$ as in 40 for the Brownian motion. Formally the approach in 41 differs slightly from our approach, as it directly uses Itô's chaos expansion from [56. To generalize [41] further along the ideas of our notes, it might be also necessary to extend Proposition A.1 to processes that have certain discontinuous trajectories. Moreover, generalizations beyond the setting of Lévy processes is left to future work. 
7 Nov 2017 05:35:26 EST

Prob+Stat

Version 2 - Submitted to Memoirs of the AMS 


\section{APPENDIX A}

\section{Technical Facts}

Let $M \neq \emptyset$ be a complete metric space that is locally $\sigma$-compact, i.e. there exist compact subsets $\emptyset \neq K_{1} \subseteq K_{2} \subseteq \ldots$, such that $\bar{\circ}_{n}=K_{n}$ and $M=\cup_{n=1}^{\infty} \stackrel{\circ}{K}_{n}$. By continuity of a stochastic process $\left(X_{x}\right)_{x \in M}: \Omega \rightarrow \mathbb{R}$ we understand that $x \mapsto X_{x}(\omega)$ is continuous for all $\omega \in \Omega$.

Proposition A.1. Let $M \neq \emptyset$ be a complete locally $\sigma$-compact metric space and $\left(X_{x}\right)_{x \in M}$ be a continuous process defined on a probability space $\left(\Omega^{0}, \mathcal{F}^{0}, \mathbb{P}^{0}\right)$, and let $\left(\beta_{x}\right)_{x \in M}$ be a stochastic process on a probability space $\left(\Omega^{1}, \mathcal{F}^{1}, \mathbb{P}^{1}\right)$ such that $X$ and $\beta$ have the same finite-dimensional distributions. Then the following is satisfied:

(1) There exists a continuous process $\left(Y_{x}\right)_{x \in M}$ on $\left(\Omega^{1}, \mathcal{F}^{1}, \mathbb{P}^{1}\right)$, which is a modification of $\left(\beta_{x}\right)_{x \in M}$, i.e. $\mathbb{P}^{1}\left(Y_{x}=\beta_{x}\right)=1$ for all $x \in M$.

(2) If there is another process $Y^{\prime}$ with this property, then $\mathbb{P}^{1}\left(Y_{x}=Y_{x}^{\prime}, x \in M\right)=1$.

(3) If $\mathcal{G}^{1} \subseteq \mathcal{F}^{1}$ is a sub- $\sigma$-algebra and $D \subseteq M$ dense, such that $\beta_{x}$ is $\mathcal{G}^{1}$-measurable for all $x \in D$, then the process $Y$ can be taken to be $\mathcal{G}^{1}$-measurable.

Proof. There is a countable set $D_{0}=\left\{a_{k}: k \geq 1\right\} \subseteq D$ such that $D_{0} \subseteq M$ is dense as well. Taking a sequence $\left(K_{n}\right)_{n=1}^{\infty}$ like in the definition of locally $\sigma$-compact we have therefore that $D_{0} \cap K_{n}$ is dense in $K_{n}$ for all $n=1,2, \ldots$

(1) and (3): We prove both parts at the same time as (1) is a special case of (3) by taking $D=M$ and $\mathcal{G}^{1}=\mathcal{F}^{1}$. Let $K$ be one of the sets $K_{n}$ and $A:=D_{0} \cap K$. Since $x \mapsto X_{x}$ is continuous on $M$, it is uniformly continuous on $K$ and $A$. Hence the set

$$
\bigcap_{n=1}^{\infty} \bigcup_{m=1}^{\infty} \bigcap_{\substack{d(u, v)<\frac{1}{m} \\ u, v \in A}}\left\{\omega:\left|X_{u}(\omega)-X_{v}(\omega)\right| \leq \frac{1}{n}\right\} \in \mathcal{F}^{0}
$$

is of $\mathbb{P}^{0}$-measure one. By the fact that $X \stackrel{d}{=} \beta$, there exists $\Omega_{0}^{1} \in \mathcal{G}^{1}$ with $\mathbb{P}^{1}\left(\Omega_{0}^{1}\right)=1$ such that $x \mapsto \beta_{x}(\omega)$ is uniformly continuous on $A$ for all $\omega \in \Omega_{0}^{1}$. Since $A$ is dense in $K$ we can define for all $x \in K$ the extension

$$
Y_{x}(\omega):=\left\{\begin{array}{rl}
\lim _{x_{n} \rightarrow x,}, \beta_{x_{n}}(\omega) & : \quad \omega \in \Omega_{0}^{1} \\
0 & : \quad \omega \in \Omega^{1} \backslash \Omega_{0}^{1}
\end{array} .\right.
$$

We obtain a $\mathcal{G}^{1}$-measurable continuous process $\left(Y_{x}\right)_{x \in K}$. Take $d \geq 1, x_{1}, \ldots, x_{d} \in$ $K$, and $a_{j, m} \in A$ with $a_{j, m} \rightarrow x_{j}$ as $m \rightarrow \infty$. Then, for $\left(t_{1}, \ldots, t_{d}\right) \in \mathbb{R}^{d}$,

$$
\begin{aligned}
\int_{\Omega^{1}} e^{i \sum_{j=1}^{d} t_{j} Y_{x_{j}}} d \mathbb{P}^{1} & =\lim _{m \rightarrow \infty} \int_{\Omega^{1}} e^{i \sum_{j=1}^{d} t_{j} \beta_{a_{j, m}}} d \mathbb{P}^{1} \\
& =\lim _{m \rightarrow \infty} \int_{\Omega^{0}} e^{i \sum_{j=1}^{d} t_{j} X_{a_{j, m}}} d \mathbb{P}^{0}
\end{aligned}
$$




$$
=\int_{\Omega^{0}} e^{i \sum_{j=1}^{d} t_{j} X_{x_{j}}} d \mathbb{P}^{0}
$$

so the finite-dimensional distributions of $Y$ and $X$ coincide. To prove $\mathbb{P}^{1}\left(Y_{x}=\right.$ $\left.\beta_{x}\right)=1$ for all $x \in K$ we check $\mathbb{P}^{1}\left(\left|Y_{x}-\beta_{x}\right|>\epsilon\right)=0$ for all $\epsilon>0$ and all $x \in K$. Let $\epsilon>0, x \in K$, and choose $\left(x_{k}\right)_{k \geq 1} \subseteq A$ such that $x_{k} \rightarrow_{k} x$. Then

$$
\begin{aligned}
\mathbb{P}^{1}\left(\left|Y_{x}-\beta_{x}\right|>\epsilon\right) & \leq \mathbb{P}^{1}\left(\left|Y_{x}-\beta_{x_{k}}\right|>\frac{\epsilon}{2}\right)+\mathbb{P}^{1}\left(\left|\beta_{x}-\beta_{x_{k}}\right|>\frac{\epsilon}{2}\right) \\
& =2 \mathbb{P}^{0}\left(\left|X_{x}-X_{x_{k}}\right|>\frac{\epsilon}{2}\right) \rightarrow_{k} 0,
\end{aligned}
$$

where we used the fact that $Y \stackrel{d}{=} X \stackrel{d}{=} \beta$ and the fact that $X$ is continuous. Thus on any compact $K_{n} \subseteq M$ we have a continuous $\mathcal{G}^{1}$-measurable process $\left(Y_{x}^{n}\right)_{x \in K_{n}}$, that is a modification of $\left(\beta_{x}\right)_{x \in K_{n}}$. Up to $\mathcal{G}^{1}$-measurable null-sets the construction is consistent in $n$ so that we can construct a $\mathcal{G}^{1}$-measurable continuous process $\left(Y_{x}\right)_{x \in M}$ (where we use $M=\bigcup_{n=1}^{\infty} \stackrel{\circ}{K}_{n}$ ) that is a modification of $\beta$.

(2) follows from the separability of $M$.

The following lemma is well-known.

Lemma A.2. Let $(A, \mathcal{A})$ be a measurable space and $M$ be a separable metric space. Assume that $f: M \times A \rightarrow \mathbb{R}$ is such that $f(x, \cdot)$ is $\mathcal{A}$-measurable for all $x \in M$ and $x \rightarrow f(x, \omega)$ is continuous for all $\omega \in A$. Then $f$ is $\mathcal{B}(M) \otimes \mathcal{A}$-measurable, where $\mathcal{B}(M)$ is generated by the open sets.

Proof. Let $\left(x_{j}\right)_{j \geq 1} \subseteq M$ be a dense set. We define for all $n, j \geq 1$

$$
B_{j}^{n}:=\left\{x \in M: d\left(x, x_{j}\right) \leq \frac{1}{n}\right\}
$$

and obtain a sequence of disjoint sets as follows: $A_{1}^{n}:=B_{1}^{n}$, and $A_{k}^{n}:=B_{k}^{n} \backslash$ $\left(\bigcup_{j=1}^{k-1} A_{j}^{n}\right)$ for $k=2,3, \ldots$ Then $M=\bigcup_{k=1}^{\infty} A_{k}^{n}$ for all $n \geq 1$. Now we define $f^{n}: M \times A \rightarrow \mathbb{R}$ as follows:

$$
f^{n}(x, \omega):=\sum_{j=1}^{\infty} f\left(x_{j}, \omega\right) 1_{A_{j}^{n}}(x) .
$$

Since $f(x, \cdot)$ is $\mathcal{A}$-measurable for all $x \in M$ and $A_{j}^{n} \in \mathcal{B}(M)$ for all $j, n \geq 1$, it follows that each $f^{n}$ is $\mathcal{B}(M) \otimes \mathcal{A}$-measurable. Moreover, for any $(x, \omega) \in M \times A$ we have the pointwise convergence $f^{n}(x, \omega) \rightarrow f(x, \omega)$ as $n \rightarrow \infty$. This follows from the facts

$$
\left|f^{n}(x, \omega)-f(x, \omega)\right|=\left|f\left(x_{j(n, x)}, \omega\right)-f(x, \omega)\right|,
$$

and $d\left(x_{j(n, x)}, x\right) \leq \frac{1}{n} \rightarrow_{n} 0$, where $j(n, x)$ is the index such that $x \in A_{j(n, x)}^{n}$.

Lemma A.3. Let $f \in L_{1}([0, T])$ be non-negative. Then

$$
\sup _{0 \leq a<b \leq T} \frac{1}{b-a} \int_{a}^{b} f(t) d t=\operatorname{esssup}_{t \in[0, T]} f .
$$


Proof. The inequality

$$
\sup _{0 \leq a<b \leq T} \frac{1}{b-a} \int_{a}^{b} f(t) d t \leq \operatorname{esssup}_{t \in[0, T]} f
$$

is obvious. According to [85, Theorem 3.3.8] there exists a Borel set $A \subseteq[0, T]$ with $\lambda(A)=T$ and $0 \leq a_{n}^{s} \leq s \leq b_{n}^{s} \leq T$ with $0<b_{n}^{s}-a_{n}^{s} \rightarrow_{n} 0$ for $s \in A$, such that

$$
\lim _{n} \frac{1}{b_{n}^{s}-a_{n}^{s}} \int_{a_{n}^{s}}^{b_{n}^{s}} f(t) d t=f(s)
$$

for all $s \in A$. Hence,

$$
f(s) \leq \sup _{0 \leq a<b \leq T} \frac{1}{b-a} \int_{a}^{b} f(t) d t
$$

for all $s \in A$.

Let $(\Omega, \mathcal{F}, \mathbb{P})$ be a complete probability space, $H$ be a separable Hilbert space with $H \neq\{0\}$, and $\left(g_{h}\right)_{h \in H}$ be an iso-normal family of Gaussian random variables $g_{h}: \Omega \rightarrow \mathbb{R}$. Assume that

$$
\mathcal{F}=\sigma\left(g_{h}: h \in H\right) \vee \mathcal{N}
$$

where $\mathcal{N}$ are the null-sets from $\mathcal{F}$. Let $\left(e_{k}\right)_{k \in I}$ be an orthonormal basis of $H$ with $I=\{1, \ldots, d\}$ or $I=\{1,2, \ldots\}$. Then

$$
\mathcal{F}=\sigma\left(g_{e_{k}}: k \in I\right) \vee \mathcal{N} .
$$

We recall that $D: \mathbb{D}_{1,2} \rightarrow L_{2}^{H}$ is a closed operator (see [70, Proposition 1.2.1]). Assume that $\varphi_{n}: \mathbb{R} \rightarrow[0, \infty) \in C_{0}^{\infty}$ such that $\varphi_{n}(x)=0$ for $x \leq 0$ and $x \geq 1 / n$ and that $\int_{\mathbb{R}} \varphi_{n}(x) d x=1$. Defining $\psi_{n}(y):=\int_{-\infty}^{y} \varphi_{n}(x) d x$, we get $\psi_{n}(x)=0$ for $x \leq 0, \psi_{n}(x)=1$ if $x \geq 1 / n$, and $0 \leq \psi_{n}(x) \leq 1$. Finally, set

$$
L_{n}(y):=\int_{-\infty}^{y} \psi_{n}(x) d x
$$

so that $L_{n}^{\prime}(x)=\psi_{n}(x) \rightarrow_{n} \chi_{(0, \infty)}(x)$ and

$$
0 \leq x-L_{n}(x) \leq \frac{1}{n}
$$

for $x \geq 0$ whereas $L_{n}(x)=0$ for $x \leq 0$. Given $\xi \in \mathbb{D}_{1,2}$ we get that $\left|\xi^{+}-L_{n}(\xi)\right| \leq$ $1 / n$ and $L_{n}^{\prime}(\xi) D \xi \rightarrow \chi_{(0, \infty)}(\xi) D \xi$ in $L_{2}^{H}$. Hence $\xi^{+} \in \mathbb{D}_{1,2}$ with

$$
D \xi^{+}=\chi_{(0, \infty)}(\xi) D \xi
$$

and, for $L>0$,

$$
D(\xi \vee(-L))=D\left((\xi+L)^{+}-L\right)=\chi_{(0, \infty)}(\xi+L) D(\xi+L)=\chi_{(-L, \infty)}(\xi) D(\xi) .
$$

From this we get

$$
D(\xi \wedge L)=-D((-\xi) \vee(-L))=-\chi_{(-L, \infty)}(-\xi) D(-\xi)=\chi_{(-\infty, L)}(\xi) D(\xi) .
$$

Finally,

$$
\begin{aligned}
D((\xi \vee(-L)) \wedge L) & =\chi_{(-\infty, L)}(\xi \vee(-L)) D((\xi \vee(-L)) \\
& =\chi_{(-\infty, L)}(\xi \vee(-L)) \chi_{(-L, \infty)}(\xi) D(\xi) \\
& =\chi_{(-L, L)}(\xi) D(\xi)
\end{aligned}
$$


Proposition A.4. Let $H$ be a separable Hilbert space with an orthonormal basis $\left(e_{k}\right)_{k \in I}$, where $I=\{1, \ldots, d\}$ or $I=\{1,2, \ldots\}$, let $\left(g_{h}\right)_{h \in H}, g_{h}: \Omega \rightarrow \mathbb{R}$, be an iso-normal family of Gaussian random variables defined on a complete probability space $(\Omega, \mathcal{F}, \mathbb{P})$ with $\mathcal{F}=\sigma\left(g_{h}: h \in H\right) \vee \mathcal{N}$ with $\mathcal{N}$ being the null-sets of $(\Omega, \mathcal{F}, \mathbb{P})$. Let $p \in[2, \infty), \varepsilon>0$, and $\xi \in \mathbb{D}_{1,2} \cap L_{p}$ such that $D \xi \in L_{p}^{H}$. Then there exist $n \geq 1$ and a bounded $f_{n} \in C^{\infty}\left(\mathbb{R}^{n}\right)$ such that all derivatives are bounded (where the bound may depend on the order of the derivative) such that for $\xi_{0}:=f_{n}\left(g_{e_{1}}, \ldots, g_{e_{n}}\right)$ one has

$$
\left\|\xi-\xi_{0}\right\|_{p}^{p}+\left\|D \xi-D \xi_{0}\right\|_{L_{p}^{H}}^{p}<\varepsilon^{p}
$$

Proof. (a) Reduction to $\operatorname{dim}(H)<\infty$ in the case $\operatorname{dim}(H)=\infty$ : Let $\mathcal{H}_{n}:=$ $\sigma\left(g_{e_{1}}, \ldots, g_{e_{n}}\right)$. By martingale convergence it follows that

$$
\lim _{n} \xi_{n}:=\lim _{n} \mathbb{E}\left(\xi \mid \mathcal{H}_{n}\right)=\xi \text { a.s. and in } L_{p} .
$$

For $n \in I$ let $P_{n}: H \rightarrow \operatorname{span}\left\{e_{1}, \ldots, e_{n}\right\} \subseteq H$ be the orthogonal projection. Then

$$
\begin{aligned}
\left\|D \xi-D \xi_{n}\right\|_{L_{p}^{H}} & =\left\|P_{n} D \xi-D \xi_{n}+\left(I-P_{n}\right) D \xi\right\|_{L_{p}^{H}} \\
& \leq\left\|P_{n} D \xi-D \xi_{n}\right\|_{L_{p}^{H}}+\left\|\left(I-P_{n}\right) D \xi\right\|_{L_{p}^{H}} .
\end{aligned}
$$

By dominated convergence,

$$
\lim _{n}\left\|\left(I-P_{n}\right) D \xi\right\|_{L_{p}^{H}}=0 .
$$

On the other hand, using $D \xi_{n}=P_{n} \mathbb{E}\left(D \xi \mid \mathcal{H}_{n}\right)$ we get

$$
\left\|P_{n} D \xi-D \xi_{n}\right\|_{L_{p}^{H}}=\left\|P_{n} D \xi-P_{n} \mathbb{E}\left(D \xi \mid \mathcal{H}_{n}\right)\right\|_{L_{p}^{H}} \leq\left\|D \xi-\mathbb{E}\left(D \xi \mid \mathcal{H}_{n}\right)\right\|_{L_{p}^{H}}
$$

that converges to zero as $n \rightarrow \infty$ because $\mathcal{F}=\vee_{n \geq 1} \mathcal{H}_{n} \vee \mathcal{N}$ and because of known facts about Banach space valued closable martingales. Summing up, we obtain

$$
\lim _{n}\left[\left\|\xi-\xi_{n}\right\|_{p}^{p}+\left\|D \xi-D \xi_{n}\right\|_{L_{p}^{H}}^{p}\right]=0
$$

(b) Reduction to a bounded $\xi$ : For $L \geq 1$ define the truncation function $\psi_{L}: \mathbb{R} \rightarrow$ $\overline{\mathbb{R}}$ by $\psi_{L}(x):=(x \vee(-L)) \wedge L$. Then

$$
\lim _{L \rightarrow \infty}\left\|\xi_{n}-\psi_{L}\left(\xi_{n}\right)\right\|_{p}=0
$$

where $\xi_{n}$ is an approximation obtained by (a) or we take $\xi_{n}=\xi$ in case $\operatorname{dim}(H)<$ $\infty$. Moreover, $\chi_{(-L, L)}\left(\xi_{n}\right) D \xi_{n}$ is a representative of $D\left(\psi_{L}\left(\xi_{n}\right)\right)$, so that

$$
\lim _{L \rightarrow \infty}\left\|D \xi_{n}-D\left(\psi_{L}\left(\xi_{n}\right)\right)\right\|_{L_{p}^{H}}=0
$$

as well. Consequently, for all $\varepsilon>0$ there are $n, L \geq 1$ such that

$$
\left\|\xi-\psi_{L}\left(\xi_{n}\right)\right\|_{p}^{p}+\left\|D \xi-D\left(\psi_{L}\left(\xi_{n}\right)\right)\right\|_{L_{p}^{H}}^{p}<\varepsilon^{p} .
$$

(c) Reduction to the smooth case: By the factorization theorem we can write

$$
\psi_{L}\left(\xi_{n}\right)=f_{n}\left(g_{e_{1}}, \ldots, g_{e_{n}}\right) \in \mathbb{D}_{1,2}
$$

for a bounded Borel function $f_{n}: \mathbb{R}^{n} \rightarrow \mathbb{R}$ where we suppress $L$ in the following. Let $F_{n}:[0,1) \times \mathbb{R}^{n} \rightarrow \mathbb{R}$ be the solution of the backward heat equation with terminal condition $f_{n}$ so that

$$
\lim _{t \rightarrow 1} F_{n}\left(t, B_{t}^{n}\right)=f_{n}\left(B_{1}^{n}\right) \quad \text { and } \quad \lim _{t \rightarrow 1} \nabla F_{n}\left(t, B_{t}^{n}\right)=D f_{n}\left(B_{1}^{n}\right)
$$


in $L_{p}$ and $L_{p}^{\mathbb{R}^{n}}$, respectively, and a.s., where $\left(B_{t}^{n}\right)_{t \in[0,1]}$ is an $n$-dimensional standard Brownian motion. But this implies also that

$$
\lim _{t \rightarrow 1} F_{n}\left(t, \sqrt{t} B_{1}^{n}\right)=f_{n}\left(B_{1}^{n}\right) \quad \text { and } \quad \lim _{t \rightarrow 1} \nabla F_{n}\left(t, \sqrt{t} B_{1}^{n}\right)=D f_{n}\left(B_{1}^{n}\right)
$$

in $L_{p}$ and $L_{p}^{\mathbb{R}^{n}}$, respectively. This can be seen from the estimate

$$
\begin{aligned}
\left\|F_{n}\left(t, \sqrt{t} B_{1}^{n}\right)-f_{n}\left(B_{1}^{n}\right)\right\|_{p}^{p} & =\mathbb{E}\left|\widetilde{\mathbb{E}} f_{n}\left(\sqrt{t} B_{1}^{n}+\widetilde{B}_{1-t}^{n}\right)-f_{n}\left(B_{1}^{n}\right)\right|^{p} \\
& \leq \mathbb{E} \widetilde{\mathbb{E}}\left|f_{n}\left(\sqrt{t} B_{1}^{n}+\widetilde{B}_{1-t}^{n}\right)-f_{n}\left(B_{1}^{n}\right)\right|^{p} \\
& =\mathbb{E} \widetilde{\mathbb{E}}\left|f_{n}\left(B_{\sqrt{t}}^{n}+\widetilde{B}_{1-\sqrt{t}}^{n}\right)-f_{n}\left(B_{1}^{n}\right)\right|^{p}
\end{aligned}
$$

so that

$$
\left\|F_{n}\left(t, \sqrt{t} B_{1}^{n}\right)-f_{n}\left(B_{1}^{n}\right)\right\|_{p} \leq 2\left\|F_{n}\left(\sqrt{t}, B_{\sqrt{t}}^{n}\right)-f_{n}\left(B_{1}^{n}\right)\right\|_{p} \rightarrow 0
$$

as $t \rightarrow 1$. The fact we used here is that $\left(F_{n}\left(t, B_{t}^{n}\right)\right)_{t \in[0,1]}$ is a martingale. As $\left(\nabla F_{n}\left(t, B_{t}^{n}\right)\right)_{t \in[0,1]}$ is a martingale as well, where we agree about $D f_{n}=: \nabla F_{n}(1, \cdot)$, the same computation yields to

$$
\left\|\nabla F_{n}\left(t, \sqrt{t} B_{1}^{n}\right)-D f_{n}\left(B_{1}^{n}\right)\right\|_{L_{p}^{H}} \leq 2\left\|\nabla F_{n}\left(\sqrt{t}, B_{\sqrt{t}}^{n}\right)-D f_{n}\left(B_{1}^{n}\right)\right\|_{L_{p}^{H}} \rightarrow 0
$$

as $t \rightarrow 1$. Letting $f_{n, t}:=F_{n}(t, \sqrt{t} \cdot)$ for $t \in[0,1)$, we get that

$$
D f_{n, t}\left(g_{e_{1}}, \ldots, g_{e_{n}}\right)=\sqrt{t} \sum_{k=1}^{n} \frac{\partial}{\partial x_{k}} F_{n}\left(t, \sqrt{t}\left(g_{e_{1}}, \ldots, g_{e_{n}}\right)\right) e_{k}
$$

because $f_{n, t} \in C_{1}^{b}\left(\mathbb{R}^{n}\right) \cap C^{b}\left(\mathbb{R}^{n}\right)$, and therefore

$$
\begin{aligned}
& \left\|D f_{n}\left(g_{e_{1}}, \ldots, g_{e_{n}}\right)-D f_{n, t}\left(g_{e_{1}}, \ldots, g_{e_{n}}\right)\right\|_{L_{p}^{H}} \\
\leq & \left\|D f_{n}\left(g_{e_{1}}, \ldots, g_{e_{n}}\right)-\sum_{k=1}^{n} \frac{\partial}{\partial x_{k}} F_{n}\left(t, \sqrt{t}\left(g_{e_{1}}, \ldots, g_{e_{n}}\right)\right) e_{k}\right\|_{L_{p}^{H}} \\
& +(1-\sqrt{t})\left\|\sum_{k=1}^{n} \frac{\partial}{\partial x_{k}} F_{n}\left(t, \sqrt{t}\left(g_{e_{1}}, \ldots, g_{e_{n}}\right)\right) e_{k}\right\|_{L_{p}^{H}} \\
\leq \| & \left\|D f_{n}\left(g_{e_{1}}, \ldots, g_{e_{n}}\right)-\sum_{k=1}^{n} \frac{\partial}{\partial x_{k}} F_{n}\left(t, \sqrt{t}\left(g_{e_{1}}, \ldots, g_{e_{n}}\right)\right) e_{k}\right\|_{L_{p}^{H}} \\
& +(1-\sqrt{t})\left\|D f_{n}\left(g_{e_{1}}, \ldots, g_{e_{n}}\right)\right\|_{L_{p}^{H}} .
\end{aligned}
$$

Summarizing,

$$
\begin{aligned}
\lim _{t \rightarrow 1}\left[\| f_{n, t}\left(g_{e_{1}}, \ldots, g_{e_{n}}\right)-\right. & f_{n}\left(g_{e_{1}}, \ldots, g_{e_{n}}\right) \|_{p}^{p} \\
& \left.+\left\|D f_{n}\left(g_{e_{1}}, \ldots, g_{e_{n}}\right)-D f_{n, t}\left(g_{e_{1}}, \ldots, g_{e_{n}}\right)\right\|_{L_{p}^{H}}^{p}\right]=0 .
\end{aligned}
$$

Lemma A.5 (Stein's martingale inequality, [66] and cf. [78, Theorem 3.2]). Let $(\Omega, \mathcal{F}, \mathbb{P})$ be a probability space, $p \in(1, \infty)$ and let $\left(\mathcal{G}_{k}\right)_{k=1}^{n}$ be an increasing sequence of sub- $\sigma$-algebras of $\mathcal{F}$. Then one has

$$
\left\|\left(\sum_{k=1}^{n}\left|\mathbb{E}\left(f_{k} \mid \mathcal{G}_{k}\right)\right|^{2}\right)^{\frac{1}{2}}\right\|_{p} \leq c_{p}\left\|\left(\sum_{k=1}^{n}\left|f_{k}\right|^{2}\right)^{\frac{1}{2}}\right\|_{p}
$$

for all $f_{1}, \ldots, f_{n} \in L_{p}$ where the constant $c_{p}>0$ depends at most on $p$. 
Note that by grouping the random variables in an appropriate way in Stein's inequality, we can also assume that $f_{1}, \ldots, f_{n}$ are random vectors with values in $\mathbb{R}^{N}$, whereas the constant $c_{p}>0$ does not enlarge.

Lemma A.6. For $p \in(1, \infty)$ assume a stochastic process $a=\left(a_{t}\right)_{t \in[0,1]}$ with values in $\mathbb{R}^{N}$ that has left-continuous paths and satisfies $\mathbb{E} \sup _{t}\left|a_{t}\right|^{p}<\infty$. Suppose a filtration $\left(\mathcal{H}_{t}\right)_{t \in[0,1]}$ and an $\left(\mathcal{H}_{t}\right)_{t \in[0,1]}$-adapted process $\left(b_{t}\right)_{t \in[0,1]}$ with values in $\mathbb{R}^{N}$ and $\mathbb{E}\left|b_{t}\right|^{p}<\infty$ for all $t \in[0,1]$ that has left-continuous paths and such that $b_{t}=$ $\mathbb{E}\left(a_{t} \mid \mathcal{H}_{t}\right)$ a.s. for $t=k / 2^{n}$ with $n=0,1,2, \ldots$ and $k=0, \ldots, 2^{n}-1$. Then one has that

$$
\left\|\left(\int_{0}^{1}\left|b_{t}\right|^{2} d t\right)^{\frac{1}{2}}\right\|_{p} \leq c \sqrt{A .5}\left\|\left(\int_{0}^{1}\left|a_{t}\right|^{2} d t\right)^{\frac{1}{2}}\right\|_{p}
$$

where $c \sqrt{A .5}>0$ is taken from Lemma A.5.

Proof. Let $t_{k}^{n}:=\frac{k}{2^{n}}$ for $n \geq 0$ and $k=0, \ldots, 2^{n}-1$. Then it follows from Lemma A.5 that

$$
\left\|\left(\sum_{k=0}^{2^{n}-1}\left(t_{k+1}^{n}-t_{k}^{n}\right)\left|\mathbb{E}\left(a_{t_{k}^{n}} \mid \mathcal{H}_{t_{k}^{n}}\right)\right|^{2}\right)^{\frac{1}{2}}\right\|_{p} \leq c \sqrt{A .5 .5}\left\|\left(\sum_{k=0}^{2^{n}-1}\left(t_{k+1}^{n}-t_{k}^{n}\right)\left|a_{t_{k}^{n}}\right|^{2}\right)^{\frac{1}{2}}\right\|_{p} .
$$

Applying twice Fatou's lemma on the left-hand side, we derive

$$
\left\|\left(\int_{0}^{1}\left|b_{t}\right|^{2} d t\right)^{\frac{1}{2}}\right\|_{p} \leq c \sqrt{A .5} \liminf _{n}\left\|\left(\sum_{k=0}^{2^{n}-1}\left(t_{k+1}^{n}-t_{k}^{n}\right)\left|a_{t_{k}^{n}}\right|^{2}\right)^{\frac{1}{2}}\right\|_{p}
$$

and we can conclude by dominated convergence.

Lemma A.7. Let $p \in(1, \infty), N \geq 1$ and $f: \mathbb{R}^{N} \rightarrow \mathbb{R} \in C^{\infty}$ where $\left\|D^{\alpha} f\right\|_{\infty}<\infty$ for all multi-indices $\alpha$. Let $\gamma_{N}$ be the standard Gaussian measure on $\mathbb{R}^{N}$. Then one has

$$
\left\|f-\int_{\mathbb{R}^{N}} f d \gamma_{N}\right\|_{L_{p}\left(\gamma_{N}\right)} \leq c \underline{\underline{\mathrm{A} .7]}}\||\nabla f|\|_{L_{p}\left(\gamma_{N}\right)}
$$

where the constant $c \sqrt{\mathrm{A} .7 \mathrm{P}}>0$ depends on $p$ only.

Proof. Let $B=\left(B_{t}\right)_{t \in[0,1]}$ be an $N$-dimensional standard Brownian motion on a complete probability space $(M, \Sigma, \mu)$ with the augmented natural filtration $\left(\mathcal{G}_{t}\right)_{t \in[0,1]}$ and that $\Sigma=\mathcal{G}_{1}$. Let

$$
F(t, x):=\mathbb{E} f\left(x+B_{1-t}\right)
$$

so that, by Itô's formula,

$$
f\left(B_{1}\right)-\mathbb{E} f\left(B_{1}\right)=\int_{0}^{1} \nabla F\left(t, B_{t}\right) d B_{t},
$$

and, by the Burkholder-Davis-Gundy inequalities,

$$
\left\|f\left(B_{1}\right)-\mathbb{E} f\left(B_{1}\right)\right\|_{p} \leq c_{p}\left\|\left(\int_{0}^{1}\left|\nabla F\left(t, B_{t}\right)\right|^{2} d t\right)^{\frac{1}{2}}\right\|_{p}
$$

and we can conclude with Lemma A.6 by $a_{t} \equiv \nabla f\left(B_{1}\right), b_{t}:=\nabla F\left(t, B_{t}\right)$ and $\mathcal{H}_{t}=\mathcal{G}_{t}$. 
We call a function $h: \Omega \rightarrow \mathbb{R}$ a $\Pi$-step-function, where $\Pi \subseteq 2^{\Omega}$ is non-empty system of subsets, provided that $h=\sum_{k=1}^{n} \alpha_{k} \chi_{A_{k}}$ for some $\alpha_{1}, \ldots, \alpha_{n} \in \mathbb{R}$ and $A_{1}, \ldots, A_{n} \in \Pi$.

THEOREM A.8. Let $\Omega$ be a non-empty set and $\Pi$ be a system of subsets of $\Omega$ such that

(i) $A, B \in \Pi$ implies $A \cap B \in \Pi$,

(ii) $\Omega \in \Pi$.

Let $p \in[1, \infty)$ and $\mathcal{F}:=\sigma(\Pi)$. Then for all $f \in L_{p}(\Omega, \mathcal{F}, \mathbb{P})$ there are $\Pi$-stepfunctions $f_{n}: \Omega \rightarrow \mathbb{R}$ such that $\lim _{n}\left\|f-f_{n}\right\|_{p}=0$.

Proof. Let $\mathcal{M}:=\left\{\chi_{A}: A \in \Pi\right\}$ so that $\mathcal{F}=\sigma(\Pi)=\sigma(\mathcal{M})$. Let $\mathcal{H}$ be the set of all bounded measurable $f: \Omega \rightarrow \mathbb{R}$ such that there exist $\Pi$-step-functions $h_{k}: \Omega \rightarrow \mathbb{R}$ with $\lim _{k}\left\|f-h_{k}\right\|_{p}=0$. Then $\mathcal{H}$ and $\mathcal{M}$ satisfy the assumptions of the monotone class theorem (see [77, p. 7]). Hence any bounded $\mathcal{F}$-measurable function can be approximated in $L_{p}$ by $\Pi$-step-functions. Our assertion follows by one more approximation obtained by truncation of a general element of $L_{p}$.

Theorem A.9. Let $X=\left(X_{t}\right)_{t \in[0, T]}, T>0, X_{t}: \Omega \rightarrow \mathbb{R}^{d}$, be a stochastic process such that all families $\left(X_{t_{i}^{k}}^{k}-X_{t_{i-1}^{k}}^{k}\right)_{k=1, i=1}^{d, N_{k}}$ with

$$
0=t_{0}^{k}<\cdots<t_{N_{k}}^{k}=T \text { and } \quad N_{k} \geq 1
$$

are independent, $\mathcal{F}:=\sigma(X)$, and $p \in[1, \infty)$. Then the following holds:

(i) The linear span of

$$
\prod_{k=1}^{d} \prod_{i=1}^{N_{k}} \chi_{\left\{X_{t_{i}^{k}}^{k}-X_{t_{i-1}^{k}}^{k} \in\left(a_{i}^{k}, b_{i}^{k}\right)\right\}},
$$

where for $N_{k}=0$ the corresponding product is replaced by 1 and for $N_{k} \geq 1$ we have $-\infty<a_{i}^{k}<b_{i}^{k}<\infty$ and $0 \leq t_{i-1}^{k}<t_{i}^{k} \leq T$, is dense in $L_{p}(\Omega, \mathcal{F}, \mathbb{P})$.

(ii) If $X$ is the d-dimensional standard Brownian motion, then the linear span of

$$
\prod_{k=1}^{d} \prod_{i=1}^{N_{k}}\left(X_{t_{i}^{k}}^{k}-X_{t_{i-1}^{k}}^{k}\right)
$$

is dense in $L_{2}(\Omega, \mathcal{F}, \mathbb{P})$, where for $N_{k}=0$ the corresponding product is replaced by 1 and for $N_{k} \geq 1$ the intervals $\left(t_{i-1}^{k}, t_{i}^{k}\right], i=1, . ., N_{k}$, are pair-wise disjoint for any fixed $k$.

Proof. (i) The system $\Pi$ consisting of $\Omega$ and all possible finite intersections of $\left\{X_{t}^{k}-X_{s}^{k} \in(a, b)\right\}$ with $k \in\{1, \ldots, d\}, 0 \leq s<t \leq T$, and $-\infty<a<b<\infty$, satisfies (i) and (ii) of Theorem A.8 and $\mathcal{F}=\sigma(\Pi)$. Therefore assertion (i) follows from the same Theorem A.8

(ii) By step (i) the random variables of form

$$
\xi=f\left(\frac{X_{t_{1}}-X_{t_{0}}}{\sqrt{t_{1}-t_{0}}}, \ldots, \frac{X_{t_{n}}-X_{t_{n-1}}}{\sqrt{t_{n}-t_{n-1}}}\right),
$$

where $n \geq 1,0=t_{0}<\cdots<t_{n}=T$ and $f: \mathbb{R}^{n d} \rightarrow \mathbb{R}$ is a bounded Borel function, are dense in $L_{2}(\Omega, \mathcal{F}, \mathbb{P})$. Exploiting the orthonormal basis of Hermite functions of $L_{2}\left(\mathbb{R}^{n d}, \gamma_{n d}\right)$ we can approximate $\xi$ by polynomials in $\left(X_{t_{i}}^{k}-X_{t_{i-1}}^{k}\right)$ 
where $k=1, \ldots, d$ and $i=1, \ldots, n$. It remains to approximate $\left(X_{b}^{k}-X_{a}^{k}\right)^{l}$ for $l \geq 2$, $k \in\{1, \ldots, d\}$ and $0 \leq a<b \leq T$ by

$$
\sum_{\substack{i_{1}, \ldots, i_{l} \in\{1, \ldots, N\} \\ \text { distinct }}}\left(X_{a+i_{1} \frac{b-a}{N}}^{k}-X_{a+\left(i_{1}-1\right) \frac{b-a}{N}}^{k}\right) \cdots\left(X_{a+i_{l} \frac{b-a}{N}}^{k}-X_{a+\left(i_{l}-1\right) \frac{b-a}{N}}^{k}\right)
$$

and $N \rightarrow \infty$.

The following lemma can be proved by the generalized Clark-Ocone formula from 71, Proposition A.1]. For completeness we include an argument based on a periodic time-shift of the Brownian motion.

LEMmA A.10. Let $p \in[2, \infty), \xi=\sum_{k=0}^{\infty} I_{k}\left(f_{k}\right) \in \mathbb{D}_{1,2} \cap L_{p}(\Omega, \mathcal{F}, \mathbb{P})$ with symmetric kernels $f_{k}$, and $b \in(0, T]$. Then there are measurable processes $\left(\mu_{t}^{b}(i)\right)_{t \in[0, b]}, i=$ $1, \ldots, d$, such that for all $a \in[0, b)$ one has

$$
\begin{aligned}
& \text { (1) }\left\|\xi-\mathbb{E}\left(\xi \mid \mathcal{G}_{a}^{b}\right)\right\|_{p} \sim_{\kappa_{p}}\left\|\left(\int_{a}^{b}\left|\mu_{r}^{b}\right|^{2} d r\right)^{\frac{1}{2}}\right\|_{p} \text {, where } \kappa_{p} \geq 1 \text { depends on } p \text { only, } \\
& \text { (2) and that } \\
& \int_{(a, b]} \mathbb{E}\left|\mu_{r}^{b}(i)-D(r, i) \xi\right|^{2} d r \\
& \quad=\int_{(a, b]} \sum_{k=1}^{\infty} k^{2}(k-1) !\left\|f_{k}((r, i), \cdot)\left[\chi_{((0, r] \cup(b, T])^{k-1}}-1\right]\right\|_{L_{2}^{k-1}}^{2} d r
\end{aligned}
$$

Proof. We represent our Wiener space by a different Brownian motion, obtained by a permutation of the original one. For this purpose we let

$$
W_{t}^{b}:= \begin{cases}W_{b+t}-W_{b} & : t \in[0, T-b] \\ W_{t-T+b}+W_{T}-W_{b} & : t \in[T-b, T]\end{cases}
$$

and obtain a standard Brownian motion (as Gaussian process). We have that $\sigma\left(W_{t}^{b}: t \in[0, T]\right)=\sigma\left(W_{t}: t \in[0, T]\right)$ and $\mathcal{G}_{t}^{b}=\mathcal{F}_{T-b+t}^{W^{b}}$. The symmetric kernels $f_{n}$ for the chaos decompositions with respect to $W$ may be transformed to $W^{b}$ as

$$
f_{n}^{b}\left(\left(t_{1}, i_{1}\right), \ldots,\left(t_{n}, i_{n}\right)\right)=f_{n}\left(\left(\left(\varphi^{b}\right)^{-1}\left(t_{1}\right), i_{1}\right), \ldots,\left(\left(\varphi^{b}\right)^{-1}\left(t_{n}\right), i_{n}\right)\right)
$$

where $\varphi^{b}(t):=t+(T-b)$ for $t \in(0, b]$ and $\varphi^{b}(t):=t-b$ for $t \in(b, T]$. Now we get that

$$
\xi-\mathbb{E}\left(\xi \mid \mathcal{G}_{a}^{b}\right)=\xi-\mathbb{E}\left(\xi \mid \mathcal{F}_{T-b+a}^{W^{b}}\right) .
$$

Let $\xi=\sum_{n=0}^{\infty} I_{n}^{b}\left(f_{n}^{b}\right)$ the chaos decomposition with respect to $W^{b}$ where the kernels are obtained from the representation in terms of $W$ by formula (53). Exploiting the representation property on the Wiener space, we find progressively measurable (with respect to the augmentation of the natural filtration $\left(\mathcal{F}_{t}^{W^{b}}\right)_{t \in[0, T]}$ of $\left.\left(W_{t}^{b}\right)_{t \in[0, T]}\right)$ processes $\left(\lambda_{t}^{b}(i)\right)_{t \in[0, T]}, i=1, \ldots, d$, satisfying $\mathbb{E} \int_{0}^{T}\left|\lambda_{t}^{b}\right|^{2} d t<\infty$ and

$$
\xi=\mathbb{E} \xi+\int_{(0, T]} \lambda_{t}^{b} d W_{t}^{b} \text { a.s. }
$$

Then the processes $\left(\mu_{t}^{b}(i)\right)_{t \in[0, b]}$ are defined by

$$
\mu_{r}^{b}(i):=\lambda_{T-b+r}^{b}(i)
$$


By the Burkholder-Davis-Gundy inequalities we get that

$$
\begin{aligned}
\left\|\xi-\mathbb{E}\left(\xi \mid \mathcal{G}_{a}^{b}\right)\right\|_{p}=\left\|\xi-\mathbb{E}\left(\xi \mid \mathcal{F}_{T-b+a}^{W^{b}}\right)\right\|_{p} \\
\\
\sim_{\kappa_{p}}\left\|\left(\int_{T-b+a}^{T}\left|\lambda_{r}^{b}\right|^{2} d r\right)^{\frac{1}{2}}\right\|_{p}=\left\|\left(\int_{a}^{b}\left|\mu_{r}^{b}\right|^{2} d r\right)^{\frac{1}{2}}\right\|_{p} .
\end{aligned}
$$

This proves part (1). Regarding part (2) it is sufficient to prove the equality for $\xi$ from a dense subset of $\mathbb{D}_{1,2}$. So we may assume $\xi=\sum_{k=1}^{N} I_{k}\left(f_{k}\right), N \geq 1$, with symmetric $f_{k}$ that are constant on dyadic cuboids of side-length $T / 2^{L}, L \geq 1$, and vanish on diagonal cuboids (where at least two edges coincide). For those $\xi$ we have the explicit formula

$$
\lambda_{t}^{b}(i)=\sum_{k=1}^{N} k I_{k-1}^{b}\left(f_{k}^{b}((t, i), \cdot) \chi_{(0, t]^{k-1}}\right)
$$

where we chose the canonical representatives on the right-hand side. In this case one can directly check part (2).

Acknowledgment: We would like to thank Christian Bender, Christel Geiss, David Nualart, Adrien Richou, and Alexander Steinicke for helpful discussions, and the referee for reading the manuscript and for his valuable comments. 
7 Nov 2017 05:35:26 EST

Prob+Stat

Version 2 - Submitted to Memoirs of the AMS 


\section{Bibliography}

[1] H. Airault, J. Ren and X. Zhang: Smoothness of local times of semimartingales. C.R. Acad. Sci. Paris 330:719-724, 2000.

[2] S. Ankirchner, P. Imkeller and G. Dos Reis: Classical and variational differentiability of BSDEs with quadratic growth. Electronic Journal Prob. 12:1418-1453, 2007.

[3] S. Ankirchner, M. Jeanblanc and T. Kruse: BSDEs with singular terminal condition and a control problem with constraints. SIAM J. Control Optim. 52(2):893-913, 2014.

[4] R. Avikainen: On irregular functionals of SDEs and the Euler scheme. Finance and Stochastics 13:381-401, 2009.

[5] R. Bañuelos and A.G. Bennett: Paraproducts and Commutators of Martingale Transforms. Proc. AMS 103:1226-1234, 1988.

[6] P. Barrieu and N. El Karoui: Monotone stability of quadratic semimartingales with applications to unbounded general quadratic BSDEs. Ann. Prob. 41:1831-1863, 2013.

[7] H. Bauer: Measure and Integration Theory. de Gruyter, 2001.

[8] F. Baumgartner and S. Geiss. Permutation Invariant Functionals of Lévy Processes. ArXiv 1407.3645, in revsion for Trans. AMS.

[9] C. Bender and R. Denk: A forward scheme for backward SDEs. Stoch. Proc. Appl. 117:1793$1812,2007$.

[10] C. Bennett and R. Sharpley: Interpolation of Operators Academic Press, 1988.

[11] J. Bergh and J. Löfström: Interpolation spaces. An Introduction. Springer, 1976.

[12] A. Bhattacharyya: On a measure of divergence between two statistical populations defined by their probability distributions. Bull. Calcutta Math. Soc. 35.99-109, 1943.

[13] J.M. Bismut: Théorie probabiliste du contrôle des diffusions. Mem. AMS 176, 1973.

[14] V.I. Bogachev: Differentiable Measures and the Malliavin Calculus. Mathematical Surveys and Monographs, Volume 164, AMS, 2010.

[15] A. Bonami and D. Lépingle: Fonction maximale et variation quadratique des martingales en présence d'un poids. Séminaire de Probabilités XIII, Univ. de Strasbourg, Lecture Notes in Mathematics 721, pp. 294-306, Springer, 1979.

[16] B. Bouchard, R. Elie and N. Touzi: Discrete-time approximation of BSDEs and probabilistic schemes for fully nonlinear PDEs. Radon Series Comp. Appl. Math 8, 1-34, 2009

[17] B. Bouchard and N. Touzi: Discrete-time approximation and Monte-Carlo simulation of backward stochastic differential equations. Stoch. Proc. Appl.111:175-206, 2004.

[18] B. Boufoussi: Régularité du temps local Brownien dans les espaces de Besov-Orlicz. Studia Math. 118(2):145-156, 1996.

[19] B. Boufoussi and B. Roynette: Le temps local Brownien appartient p.s. à l'espace de Besov $\mathbb{B}_{p, \alpha}^{1 / 2}$. C.R. Acad. Sci. Paris Série I 316:843-848, 1993.

[20] P. Briand and F. Confortola: BSDEs with stochastic Lipschitz condition and quadratic PDEs in Hilbert spaces. Stoch. Proc. App. 118:818-838, 2008.

[21] P. Briand, B. Delyon, Y. Hu, E. Pardoux and L. Stoica: $L_{p}$ solutions of backward stochastic differential equations. Stoch. Proc. Appl. 108:109-129, 2003.

[22] P. Briand and R. Elie: A simple constructive approach to quadratic BSDEs with or without delay. Stoch. Proc. App. 123:2921-2939, 2013.

[23] P. Briand and Y. Hu: BSDEs with quadratic growth and unbounded terminal value. Probab. Theory Relat. Fields 136:604-618, 2006.

[24] P. Briand and Y. Hu: Quadratic BSDEs with convex generators and unbounded terminal conditions. Probab. Theory Relat. Fields 141:543-567, 2008.

[25] P. Briand, J.-P. Lepeltier and J.S. Martin: One-dimensional backward stochastic differential equations whose coefficient is monotone in $y$ and non-Lipschitz in $z$. Bernoulli 13:80-91, 2007. 
[26] J.-F. Chassagneux and A. Richou: Numerical simulation of quadratic BSDEs. Ann. Appl. Prob. 26:262-304, 2016.

[27] P. Cheridito and K. Nam: BSDEs with terminal conditions that have bounded Malliavin derivative. J. Funct. Anal. 266:1257-1285, 2014.

[28] P. Cheridito and K. Nam: Multidimensional quadratic and subquadratic BSDEs with special structure. Stochastics: An International Journal of Probability and Stochastic Processes 87:871-884, 2015.

[29] F. Coquet, Y. Hu, J. Memin and S. Peng: Filtration-consistent nonlinear expectations and related g-expectations. Prob. Theory Relat. Fields 123:1-27, 2002.

[30] F. Delbaen, Y. Hu and A. Richou: On the uniqueness of solutions to quadratic BSDEs with convex generators and unbounded terminal conditions. Ann. Inst. Henri Poincar Probab. Stat. 47:559-574, 2011.

[31] F. Delbaen, Y. Hu and A. Richou: On the uniqueness of solutions to quadratic BSDEs with convex generators and unbounded terminal conditions: the critical case. Discrete Contin. Dyn. Syst. 35:5273-5283, 2015.

[32] F. Delbaen and S. Tang: Harmonic analysis of stochastic equations and backward stochastic differential equations. Prob. Theory Relat. Fields 146:291-336, 2010.

[33] W. E, M. Hutzenthaler, A. Jentzen and T. Kruse: On multilevel Picard numerical approximations for high-dimensional nonlinear parabolic partial differential equations and highdimensional nonlinear backward stochastic differential equations. ArXiv:1708.03223.

[34] N. El Karoui, S. Peng and M.C. Quenez: Backward stochastic differential equations in finance. Mathematical Finance 7:1-71, 1997.

[35] M. Emery: Stabilité des solutions des équations différentielles stochastiques; applications aux intégrales multiplicatives stochastiques. Z. Wahrscheinlichkeitstheorie Verw. Gebiete 41:241$262,1978$.

[36] M. Emery: Équations différentielles stochastiques lipschitziennes: étude de la stabilité. Séminaire de Probabilités XIII. Lecture Notes in Mathematics 721, pp. 281-293. Springer, 1979.

[37] C. Frei: Splitting multidimensional BSDEs and finding local equilibria. Stoch. Proc. Appl. 124:2654-2671, 2014.

[38] A.M. Garsia: Martingale Inequalities. W.A. Benjamin, 1973.

[39] C. Geiss and S. Geiss: On approximation of a class of stochastic integrals and interpolation. Stochastics and Stochachstics Reports 76(4):339362, 2004.

[40] C. Geiss, S. Geiss and E. Gobet: Generalized fractional smoothness and $L_{p}$-variation of BSDEs with non-Lipschitz terminal condition. Stoch. Proc. Appl. 122:2078-2116, 2012.

[41] C. Geiss and A. Steinicke. $L_{2}$-variation of Lévy driven BSDEs with non-smooth terminal conditions. Bernoulli, 22(2):995-1025, 2016.

[42] C. Geiss and A. Steinicke: Malliavin derivative of random functions and applications to Lévy driven BSDEs. ArXiv:1404.4477v4.

[43] S. Geiss: Quantitative approximation of certain stochastic integrals. Stochastics and Stochachstics Reports 73(3-4):241270, 2002.

[44] S. Geiss: Weighted BMO and discrete time hedging within the Black-Scholes model. Prob. Theory Related Fields 132:39-73, 2005.

[45] S. Geiss and M. Hujo: Interpolation and approximation in $L_{2}(\gamma)$. J. Approx. Theory 144:213232, $200 \%$.

[46] S. Geiss and A. Toivola: On fractional smoothness and $L_{p}$-approximation on the Gaussian space. Ann. Prob. 43:605-638, 2015.

[47] E. Gobet, J.-P. Lemor, X. Warin: A regression-based Monte Carlo method to solve backward stochastic differential equations. Ann. Appl. Prob. 15:2172-2202, 2005.

[48] E. Gobet and A. Makhlouf: $L_{2}$-time regularity of BSDEs with irregular terminal functions. Stoch. Proc. Appl. 120:1105-1132, 2010.

[49] P. Grandits: On a conjecture of Kazamaki. Séminaire de Probabilités XXX. Lecture Notes in Mathematics 1626, pp. 357-360. Springer, 1996.

[50] F. Hirsch: Lipschitz functions and fractional Sobolev spaces. Potential Analysis 11:415-429, 1999.

[51] Y. Hu, P. Imkeller and M. Müller: Utility maximization in incomplete markets. Ann. Appl. Prob. 15:1691-1712, 2005. 
[52] Y. Hu, D. Nualart and X. Song: Malliavin Calculus for backward stochastic differential equations and applications to numerical solutions. Ann. Appl. Prob. 21:2379-2423, 2011.

[53] P. Imkeller: Malliavin's calculus and applications in stochastic control and finance. IM PAN Lecture Notes, Warsaw 2009.

[54] P. Imkeller and G. dos Reis: Path regularity and explicit convergence rate for BSDE with truncated quadratic growth. Stoch. Proc. Appl. 120:348-379, 2010.

[55] P. Imkeller, G. dos Reis, and J. Zhang: Results on numerics for FBSDE with drivers of quadratic growth. Contemporary Quantitative Finance, Springer Berlin, 2010.

[56] K. Itô: Spectral type of the shift transformation of differential processes with stationary increments. Trans. AMS, 81(2): 253-263, 1956.

[57] M. Izumisawa and N. Kazamaki: Weighted norm inequalities for martingales. Tôhoku Math. Journal 29:115-124, 1977.

[58] I. Karatzas and S. Shreve: Brownian Motion and Stochastic Calculus. 2nd Edition. Springer, 1991.

[59] N. Kazamaki: Continuous Exponential Martingales and BMO. Lecture Notes in Mathematics 1579. Springer, 1994.

[60] M. Kobylanski: Backward stochastic differential equations and partial differential equations with quadratic growth. Ann. Prob. 28:558-602, 2000.

[61] J.-P. Lepeltier and J.S. Martin: Existence for BSDE with superlinear- quadratic coefficient. Stochastics and Stochastics Reports 63:227-240, 1998.

[62] J.-P. Lepeltier and J.S. Martin: On the existence or non-existence of solutions for certain backward stochastic equations. Bernoulli 8:123-137, 2002.

[63] A. Lionnet, G. dos Reis and L. Szpruch: Time discretization of FBSDE with polynomial growth drivers and reaction-diffusion PDEs Ann. Appl. Prob. 25:2563-2625, 2015.

[64] J. Ma, J. Yong: Forward-Backward Stochastic Differential Equations and their Applications. Lecture Notes in Mathematics 1702, Springer.

[65] P. Malliavin, H. Airault, L. Kay and G. Letac: Integration and Probability. Springer, 1995.

[66] P.A. Meyer: Martingales locales fonctionnelles additives (II). Séminaire de probabilités de Strasbourg 12:786-803, 1978.

[67] M. Mocha and N. Westray: Quadratic semimartingale BSDEs under an exponential moments condition. Séminaire de Probabilités XLIV. Lecture Notes in Mathematics, 2046, pp 105-139. Springer, 2012.

[68] M.-A. Morlais: Quadratic BSDEs driven by a continuous martingale and applications to the utility maximization problem. Finance Stoch. 13:121-150, 2009.

[69] K. Nam: Backward stochastic differential equations with superlinear drivers. PhD Thesis. Princeton University, 2014.

[70] D. Nualart: The Malliavin Calculus and Related Topics. 2nd edition, Springer, 2006.

[71] D. Nualart and E. Pardoux: Stochastic calculus with anticipating integrands. Probab. Theory Relat. Fields 78:535-581, 1988.

[72] D. Nualart and J. Vives: Smoothness of local time and related Wiener functionals. Potent. Anal. 1:257-263, 1992.

[73] E. Pardoux and S. Peng: Adapted solutions of backward stochastic equations. System and Control Lett. 14:55-61, 1990.

[74] S. Peng: Backward SDE and related g-expectation. In Backward Stochastic Differential Equations, Pitman Research Notes in Math. No. 364, ed. by N. El Karoui and L. Mazliak, 141-159, 1997.

[75] S. Peng: Nonlinear expectations and nonlinear Markov chains. Chin. Ann. Math. 26B(2):159$184,2005$.

[76] A. Popier: Backward stochastic differential equations with singular terminal condition. Stoch. Proc. Appl. 116 (12):2014-2056, 2006.

[77] P.E. Protter: Stochastic Integration and Differential Equations. 2nd edition, Springer, 2004.

[78] Y. Qiu: On the UMD-constants for a class of iterated $L_{p}\left(L_{q}\right)$ spaces. J. Funct. Anal. 263:2409$2429,2012$.

[79] G. Dos Reis: On some properties of solutions of quadratic growth BSDE and applications in finance and insurance. PhD Thesis, Humboldt University Berlin, 2010.

[80] D. Revuz and M. Yor: Continuous martingales and Brownian motion. Springer, 1999.

[81] A. Richou: Numerical simulation of BSDEs with drivers of quadratic growth. Ann. Appl. Prob. 21:1933-1964, 2011. 
[82] A. Richou: Markovian quadratic and superquadratic BSDEs with an unbounded terminal condition. Stoch. Proc. Appl. 122:3173-3208, 2012.

[83] W. Schachermayer: A characterisation of the closure of $H^{\infty}$ in BMO. Séminaire de Probabilités, XXX. Lecture Notes in Mathematics, 1626, pp. 344-356. Springer, 1996.

[84] D.W. Stroock: Applications of Fefferman-Stein type interpolation to probability theory and analysis. Comm. Pure Appl. Math. 26:477-495, 1973 Springer, 2011.

[85] D.W. Stroock: Essentials of Integration Theory for Analysis. Springer, 2011.

[86] H. Triebel: Interpolation Theory, Function spaces, Differential Operators. North-Holland, 1978.

[87] P. Turkeddjiev: Two algorithms for the discrete time approximation of Markovian backward stochastic differential equations under local conditions. Electronic Journal Prob. 20(50):1-49, 2015.

[88] S. Watanabe: Fractional order Sobolev spaces on Wiener space. Probab. Theory Relat. Fields 95:175-198, 1993.

[89] P. Xie and X.C. Zhang: Fractional smoothness of some stochastic integrals. Acta Mathematica Sinica 23:1053-1058, 2007.

[90] J. Ylinen: Decoupling on the Wiener space and variational estimates for BSDEs. PhD Thesis. Report 148 of the Department of Mathematics and Statistics, University of Jyväskylä, 2015.

[91] J. Ylinen: Weighted bounded mean oscillation applied to backward stochastic differential equations. ArXiv:1501.01183v2.

[92] J. Zhang: A numerical scheme for BSDEs. Ann. Appl. Prob. 14:459-488, 2004.

[93] J. Zhang: Representation of solutions to BSDEs associated with a degenerate FSDE. Ann. Appl. Prob. 15:1798-1831, 2005. 


\section{Index}

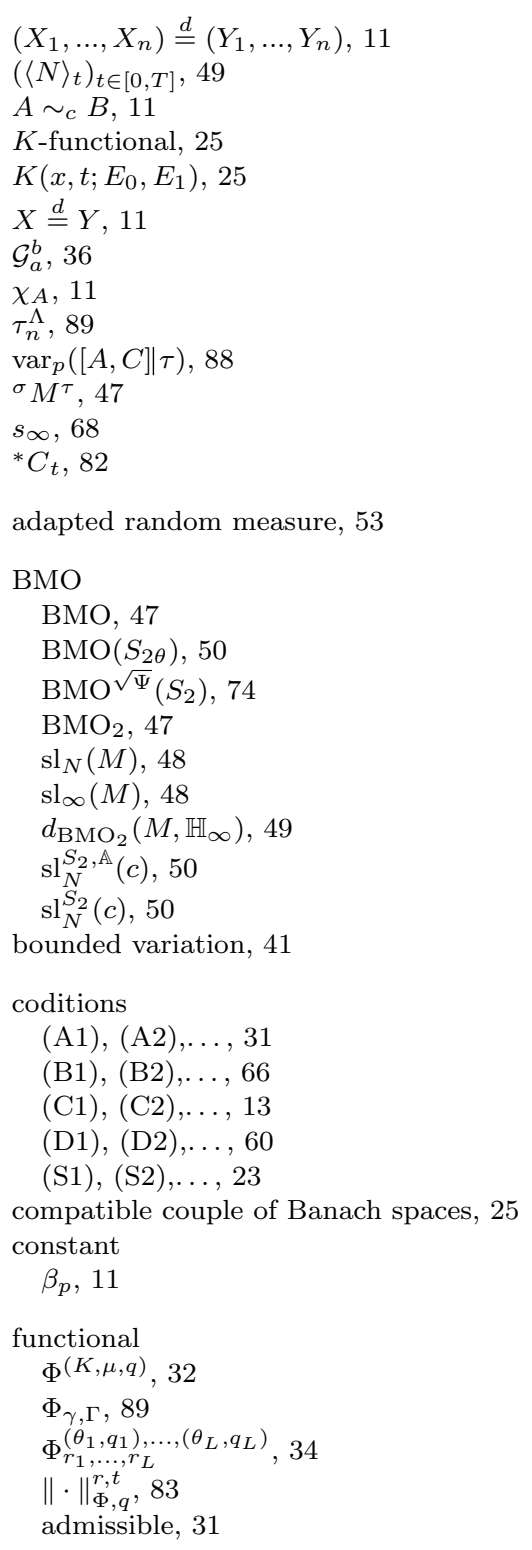

consistent, 14

Hermite polynomial, 26

inequality

$\mathcal{R H}_{\beta}(\lambda), 58$

Fefferman's inequality, 55

reverse Hölder inequality, 58

Stein's inequality, 99

local time

$L_{T}^{\alpha}, 45$

$N_{T}^{\alpha}, 43$

locally $\sigma$-compact, 17,95

Malliavin derivative, 27

operator

$\mathcal{C}, 14$

$\mathcal{C}^{M}, 17$

$\mathcal{C}_{0}, 14$

$\mathcal{C}_{T}, 17$

sliceable, 48

space

$\left(E_{0}, E_{1}\right)_{\theta, q}, 25$

$(\Delta, \delta), 31$

$\left(\Omega_{T}, \Sigma_{T}, \mathbb{P}_{T}\right), 17$

$(\mathfrak{D}, \delta), 29$

$B_{p, q}^{\theta}, 27$

$E_{\rho}, 51$

$L_{0}(A ; C(M)), 17$

$L_{0}(\Omega, \mathcal{F}, \mathbb{P}), 11$

$L_{\infty}\left([0, T] ; L_{p}(\Omega)\right), 35$

$L_{p}(\Omega, \mathcal{F}, \mathbb{P}), p \in(0, \infty], 11$

$L_{p}^{*}\left(\Omega ; L_{2}([0, T])\right), 35$

$L_{p}^{X}, 26$

$L_{\text {exp }}, 51$

$L_{\exp _{\alpha}, 76}$

$\mathbb{B}_{p}^{\Phi_{\gamma, \Gamma}, 89}$

$\mathbb{B}_{p}^{\Phi}, 31$

$\mathbb{B}_{p, q}^{\theta}\left(\mathbb{R}^{n}, \gamma_{n}\right), 27$

$\mathbb{D}_{1, p}, 27$

$\mathbb{H}_{\infty}, 49$

$\mathbb{H}_{p}\left(S_{2}\right), 52$ 
cExp, 77

$\operatorname{cExp}(\eta, \mu), 7677$

$\mathcal{L}_{0}(A ; C(M)), 17$

$\mathcal{L}_{p}^{X}, 26$

$\mathbb{B}_{p}^{\Phi_{r}}, 41$

$\mathcal{L}_{0}(\Omega, \mathcal{F}, \mathbb{P}), 10$

$\mathcal{L}_{p}(\Omega, \mathcal{F}, \mathbb{P}), p \in(0, \infty], 10$

Banach function norm, 51

Banach function space, 51

real interpolation space, 25

Wiener chaos, 26

Wiener chaos expansion, 27 